\title{
Prevention and early detection of sexually transmitted diseases
}

Citation for published version (APA):

Leenaars, P. E. M. (1994). Prevention and early detection of sexually transmitted diseases. [Doctoral Thesis, Maastricht University]. Thesis Publishers. https://doi.org/10.26481/dis.19940101pl

Document status and date:

Published: 01/01/1994

DOI:

10.26481/dis.19940101pl

Document Version:

Publisher's PDF, also known as Version of record

\section{Please check the document version of this publication:}

- A submitted manuscript is the version of the article upon submission and before peer-review. There can be important differences between the submitted version and the official published version of record.

People interested in the research are advised to contact the author for the final version of the publication, or visit the DOI to the publisher's website.

- The final author version and the galley proof are versions of the publication after peer review.

- The final published version features the final layout of the paper including the volume, issue and page numbers.

Link to publication

\footnotetext{
General rights rights.

- You may freely distribute the URL identifying the publication in the public portal. please follow below link for the End User Agreement:

www.umlib.nl/taverne-license

Take down policy

If you believe that this document breaches copyright please contact us at:

repository@maastrichtuniversity.nl

providing details and we will investigate your claim.
}

Copyright and moral rights for the publications made accessible in the public portal are retained by the authors and/or other copyright owners and it is a condition of accessing publications that users recognise and abide by the legal requirements associated with these

- Users may download and print one copy of any publication from the public portal for the purpose of private study or research.

- You may not further distribute the material or use it for any profit-making activity or commercial gain

If the publication is distributed under the terms of Article $25 \mathrm{fa}$ of the Dutch Copyright Act, indicated by the "Taverne" license above, 
PREVENTION AND EARLY DETECTION OF SEXUALLY TRANSMITTED DISEASES 


\section{Promotor:}

Prof. Dr. G.J. Kok

\section{Co-promotor:}

Dr. R. Rombouts (Rijksuniversiteit Groningen)

\section{Beoordelingscommissie:}

Prof. Dr. P.G. Knipschild (voorzitter)

Prof. Dr. R.A. Coutinho (Universiteit van Amsterdam)

Dr. F. Nijhuis

Prof. Dr. G.S. Parcel (University of Texas, Houston)

Dr. G.A.M. Widdershoven

CIP-DATA KONINKLIJKE BIBLIOTHEEK, DEN HAAG

Leenaars, Ellie

Prevention and early detection of Sexually Transmitted Diseases I

Ellie Leenaars. - Amsterdam : Thesis Publishers

Also publ. as thesis Maastricht, 1994. - With ref. - With summary in Dutch. ISBN $90-5170-267-1$

NUGI $735 / 715$

Subject headings: sexually transmitted diseases ; prevention

\section{- 1994 by P.E.M. Leenaars}

All rights reserved. No part of this book may be reproduced in any form by print, photoprint, microfilm or any other means, without written permission of the author.

A part of this research project was supported by the Dutch Ministry of Welfare. Public Health and Culture.

Cover: Hanne Lijesen

Lay-out: Marianne Oldenborg

Production: Thesis Publishers, Amsterdam 
To my husband Sipke $W$. van der Werf and my children

Gotske Anne, Juliëtte and Haye Majoor For my parents 


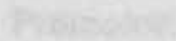

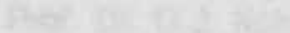

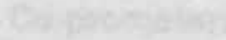

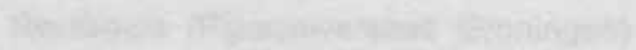

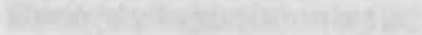

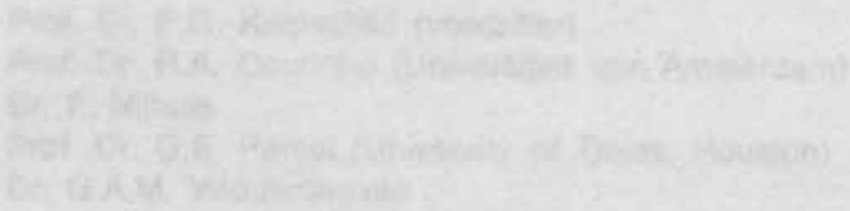

The

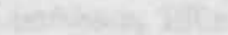

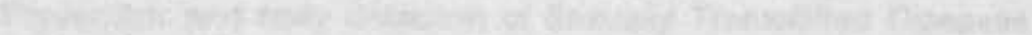

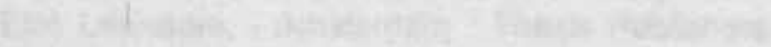

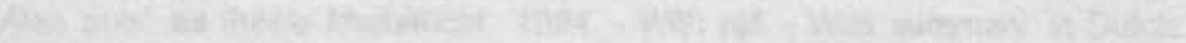

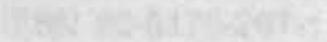

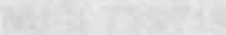

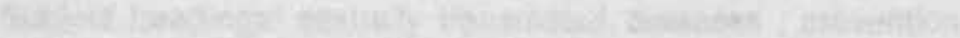

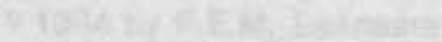

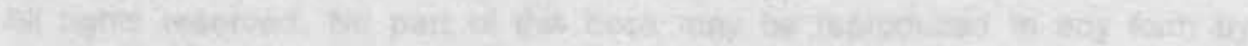

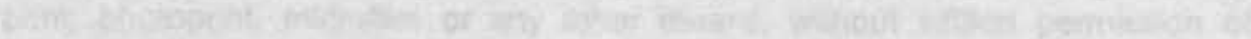

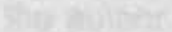

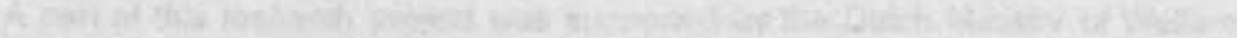

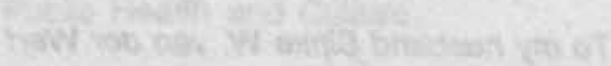

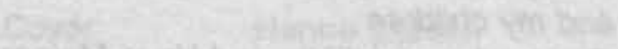

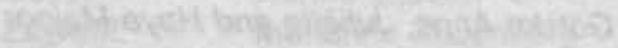

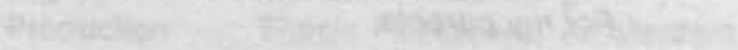


CONTENTS

ACKNOWLEDGMENTS

GENERAL OUTLNE

PART I

\section{OVERVIEW}

PLANNING HEALTH PROMOTION IN STDE: THE PRECEDEPAOCEED MODEL

1.1 Social diagnosis

1.1.1 Identilying STDs as an urgent health problern

1.2 Epidemiological diagnosis

1.2.1 Classification and characteristics of major STDs

1.2.2 Trends of major STDs

1.2.3 The incidence of major complications of STDs

1.2.4 Risk factors for contracting STDs and for developing complications

1.3.1 Assessment of behavioural factors pertaining to contracting STDs

1.3.2 Assessment of behavioural factors pertaining to the development of complications

1.3.3 Environmental factors influencing STDs and STD-related complications

1.4 Educational and organisational diagnosis

1.4.1 Social psychological models of human behaviour

1.4.2 Social psychological models for health behaviour

1.4.3 Predisposing, Enabling and Reinforcing factors

1.4.4 The application of Predisposing. Enabling and Reinforcing factors on STD-preventive behaviour

1.4.5 The application of Predisposing, Enabling and Reinforcing on early STD detection

1.5.1 Research questions 
2.1 A summary of major findings

2.1.1 Determinants of STD-preventive behaviour

2.1.2 Determinants of early STD detection behaviour

2.1.3 The role of service attributes in the choice of circuits

2.2 Methodological issues

2.2.1 Limitations of the studies

71

2.2.2 Methodological remarks

2.3 Discussion and recommendations

2.3.1 STD prevention: education and promotion of STD-preventive behaviour

2.3.2 Early STD detection: education and promotion of early detection behaviour

2.3.3 Patient care: the importance of special service attributes in STD health care

2.3.4 Theoretical conclusions and future research

PART II

\section{DETERMINANTS OF STD-PREVENTIVE BEHAVIOUR}

3 SEXUAL BEHAVIOUR AND DETERMINANTS OF CONDOM USE AMONG HETEROSEXUALS REQUESTING AN HIV TEST

3.1 Introduction

3.2 Methods

3.3 Results

3.4 Discussion

DETERMINANTS OF CONDOM USE AMONG ADOLESCENTS

4.1 Introduction

4.2 Methods

4.3 Results

4.4 Discussion

BELIEFS, EVALUATIONS AND THE PREVENTION OF STD. AMONG SECONDARY SCHOOL PUPILS

5.1 Introduction

5.2 Methods

5.3 Results

5.4 Discussion 
PART III

6

7

8

9

\section{DETERMINANTS OF EARLY DETECTION} BEHAVIOUR

STD-RELATED HEALTH BEHAVIOUR: DETERMINANITS OF EARLY STD DETECTION

6.1 Introduction

127

6.2 Methods

128

6.3 Results

132

6.4 Discussion

SEEKINO MEDICAL CARE FOR A SEXUALLY TRANSMITTED DISEASE: DETERMINANTS OF DELAY BEHAVIOUR

7.1 Introduction

7.2 Methods

7.3 Results

144

7.4 Discussion

SERVICE ATTRIBUTES AND THE CHOICE OF STD HEALTH SERVICES IN PERSONS SEEKING A MEDICAL EXAMINATION FOR AN STD

8.1 Introduction

8.2 Methods

8.3 Results

8.4 Discussion

CONSUMER BEHAVIOUR OF ADOLESCENTS WHEN SUSPECTING A SEXUALIY TRANSMITTED DISEASE

9.1 Introduction

9.2 Methods

174

9.3 Results

176

9.4 Discussion

REFERENCES

SUMMARY IN ENGUSH

SUMMARY IN DUTCH 


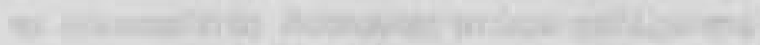

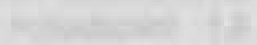

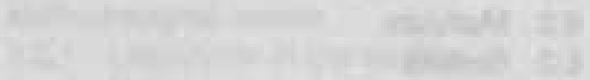

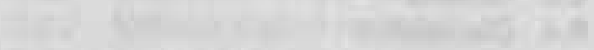

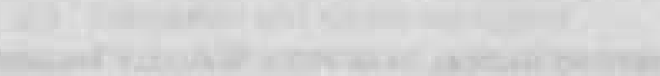

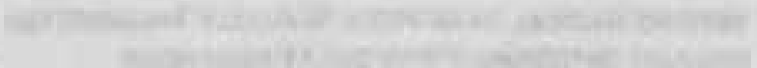

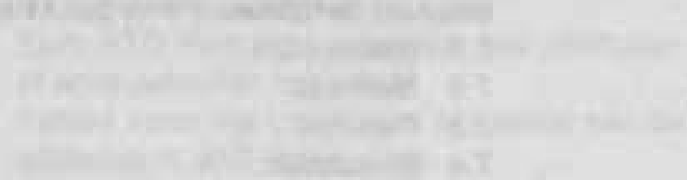

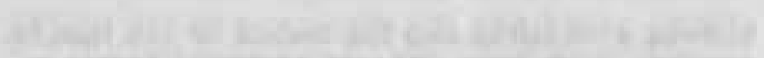

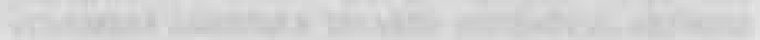

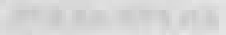

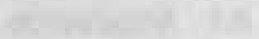

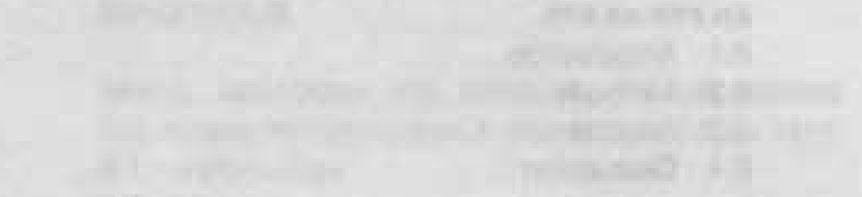

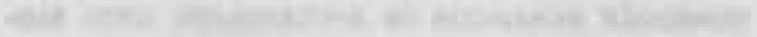
and

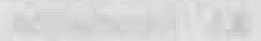

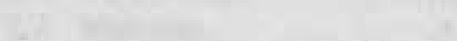




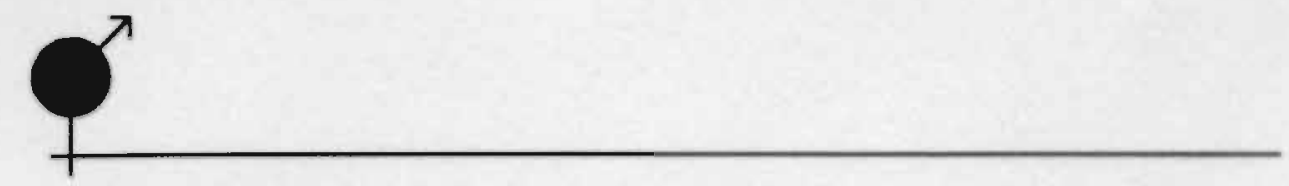

\section{ACKNOWLEDGMENTS}

On behalf of the research described in this thesis, many people have helped me in different ways.

First, I am greatly indebted to my co-promotor Rene Rombouts. He supervised me and helped me to write proposals, abstracts, articles and the underlying thesis. Dear Rene, I have appreciated your critical comments throughoul the years.

I also wish to express my appreciation to Gerjo Kok. The research was going on for quite some time when you became involved. Whit your stimulating conversations, suggestions and criticism it would have been impossible to finish thle project.

A special debt of gratitude goes to Anne-Marle de Jong who read and re-read all drafte in order to correct my English property. I aleo want to thank Marianne Oldenborg for her valuable advice with respect to the ley-out of this thesis. Anne-Merle and Marianne, your spontaneous readiness to help me has made thls part of the writing very agreeable.

Besides these advisors other pecple contributed as well. The research which ls described In this book was carried out the Department of Health Science and Epidemiology, Faculty of Medicine of the University of Utrecht. I am greatly indebted to my collesques Gerdien de Weert, Adriana Oliehoek and Eeije Spithoven who assisted In the realisation of the projects. I would also like to thank Guus Schrijvers for his proposals for the planning and organisation of the research.

I am also moet greteful to the Dutch Minlstry of Welfare, Health and Culture and the Dutch STD-Foundation, in particular Wendy Reymerink and Marla Paalman for providing research orders. They can be considered as the impulse for writing this book about prevention and early detection of Sexually Transmitted Diseases.

Less concrete, but at least as important, were the critical comments of Dlederick Boeykens, Marita van de Laar and Albèr Pennings. I would like to express my grattude for all the suggestions they made.

Finally, I would like to gratefully thank the general practitioners, health care providere from STD clinics, Municipal Health Services and Family Planning Clinics, and the social nurses for their encouraging the subjects to participate in the various studies. Without their contribution and the willingness of the subjects to complete the questionnaires, my research would simply have been impossible.

Leidschendam, september 1993

Ellis Leenaars 


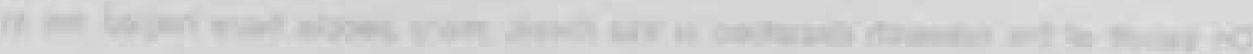

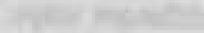

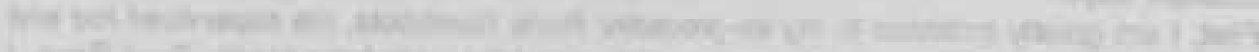

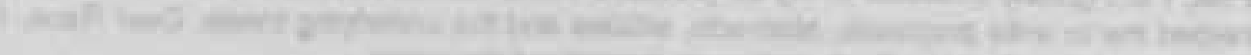

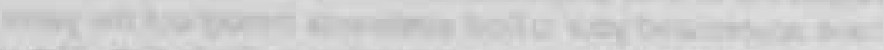

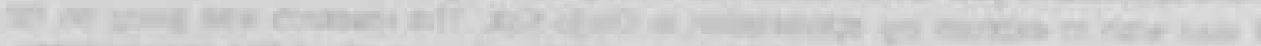

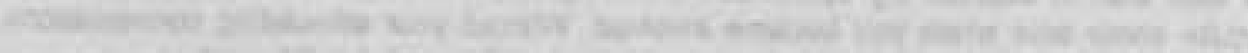

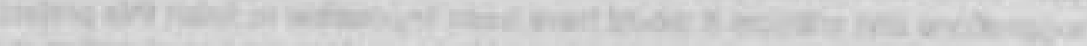

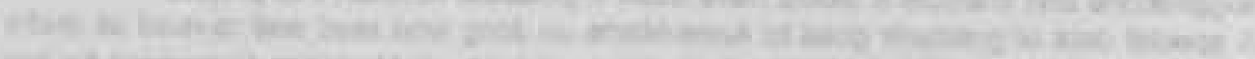

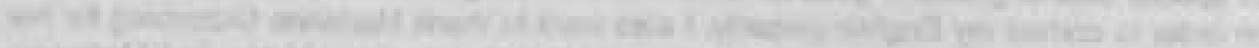

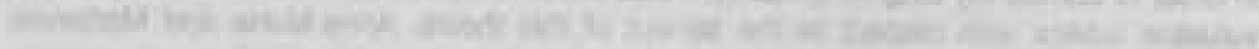

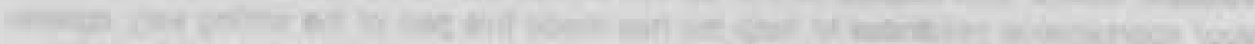

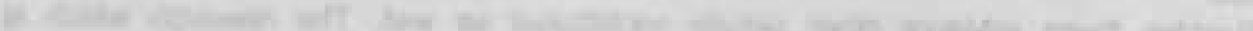

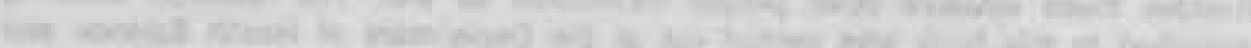
W W

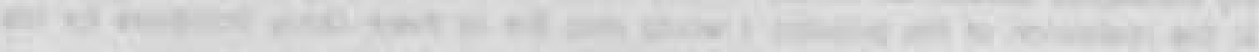
that

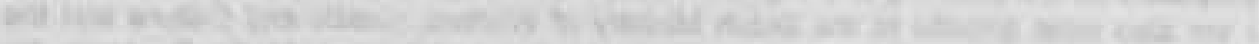

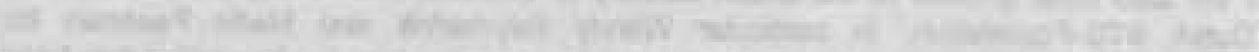

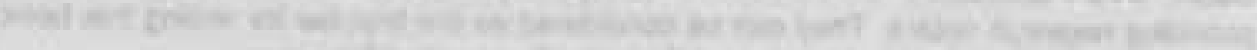
.

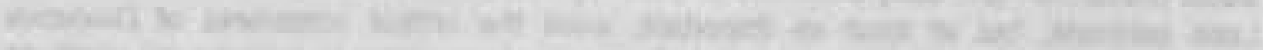

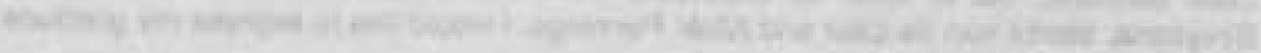

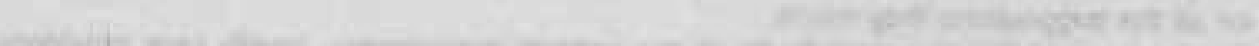
Chan

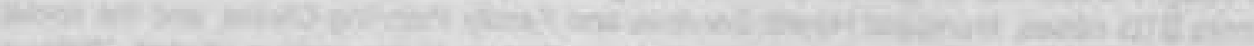

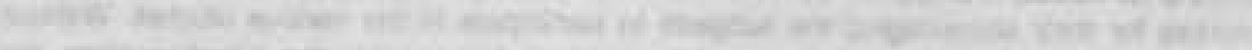

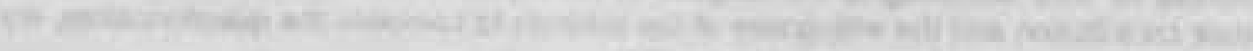

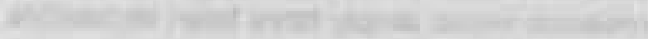

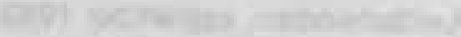

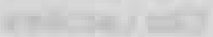




\section{GENERAL OUTLINE}

Sexually Transmitted Diseases (STDs) are defined as a group of communicable diseases which are predominantly transferred by sexual contact (Holmes, 1990). Transmission of the Hurnan Immunodeficiency Virus (HIV), the virus which causes the Acquired Immunodeficiency Syndrome (AIDS), is known to occur through sexual contact with infected individuals, after the exposure to infected blood or bloodproducts and perinatally (Friedland \& Klein, 1987). The prevalence of STDs has reached a disturbing level in many countries and the complications of untreated STDs (e.g. Pelvic Inflammatory Disease) not only have serious consequences for the individual and their family but also for the community. Moreover, the significance of STDs is not only related to the number of cases infected but also to its social, emotional and economic impact.

From this perspective the World Health Organization (WHO, 1985) stated that STD control must have two main aims:

- to interrupt the transmission of infections;

- to prevent the development of complications and sequelae.

The first aim is directed at primary prevention of STDs by STD risk-reduction behaviours and the second aim has to do with early detection of STDs, such as STD selfexamination, periodic clinical examination and prompt help-seeking behaviour. In addition, Meheus (1990) suggested that these aims can be accomplished by:

- reducing exposure to infection by educating persons at risk to reduce the number of sex partners and to avoid sexual intercourse with persons who have a high probability of being infected;

- preventing infection by promoting the use of condoms or other prophylactic barriers;

- detecting and curing diseases by implementing disease detection activities, providing adequate diagnostic and treatment facilities, and promoting help-seeking behaviour;

- limiting complications and turther transmission of infection by providing early and appropriate treatment for symptomatic and asymptomatic patients and their sex partner(s) and through counselling.

This thesis focuses on health promotion aimed at the prevention of STDs, including HIV infection and their late seguelae as a combined strategy for primary prevention, early detection and proper patient care. Health promotion, as defined by Green and Kreuter (1991) and their proposed framework for planning, intervention and implementation strategies (THE PRECEDE-PROCEED MODEL) forms the perspective for this thesis. This framework has been used successfully in planning health promotion activities for varying subjects (Green \& Kreuter, 1991). According to the model, PRECEOE takes into account the multiple factors that shape health status and helps the planner arrive at a subset of factors as targets for intervention. PROCEED provides additional steps for developing policy and initiating the implementation and evaluation process. As mentioned in their framework PRECEOE-PROCEED consists of nine basic phases (see chapter 1). Because of the central focus of this study on determinants of STD-preventive behaviours and behaviours aimed at early STD detection, the application of phases 1 to 4 will be dis- 
cussed in Chapter 1. Because of this central attention, phases 5 to 9 will not be presented in this thesis. The book itself is divided into three parts.

PART I, a general overview, will discuss the most essential points of a literature study Into STDs and the main results from our studies, followed by recommendations on different matters within the field of STD prevention and early STD detection. Before going any further, it should be pointed out that the models used in PART I enclose a variety of variables to explain health behaviour. These variables are not only discussed In the literature study, but also in the investigations we conducted. We therefore advice the reader to keep an eye on the continous thread of this thesis. Those readers interested in further information are also referred to the respective chapters of our studies that will be presented in parts II and III.

Chapter 1 describes the PRECEDE-PROCEED model and the application of the first part of this model to Sexually Transmitted Diseases. After a general introduction of the PRECEDE-PROCEED model, a social diagnosis is presented in terms of the identification of STDs as an urgent health problem. Special attention is paid to the effects of STDs and STD-related complications on the quality of life of the Dutch population.

Paragraph 1.2 gives detailed information about the epidemiological diagnosis and includes a classification and the characteristics of major STDs, trends of major STDs and HIV-infection, and a description of the incidence of a major complication of STDs, namely Pelvic Inflammatory Diseases (PID) in women. Finally, non-behavioural risk factors for acquiring STDs and for developing STD-related complications are distinguished.

Apart from these epidemiological findings a behavioural and environmental diagnosis of STDs is described in $\$ 1.3$. Firstly, behavioural risk factors for acquiring STDs are discussed, followed by a description of the high-risk sexual behaviours and behaviours aimed at preventing transmission of STDs. Attention is paid to the separate high-risk groups, their high-risk sexual behaviour and their preventive behaviours. Secondly, a description of behavioural risk factors for the development of STD-related complications and behaviours aimed at the early detection are presented, such as: STD self-examination, periodic clinical examination and prompt help-seeking behaviour. Thirdly, attention is paid to factors in the social and physical environment other than specific individual actions that could be linked causally to both the occurrence of STDs and STD-related complications.

In order to influence STD-preventive behaviours such as various risk-reduction behaviours and early STO detection activities, their respective determinants must be known. Therefore, an educational and organisational diagnosis is first presented broadly in § 1.4. Social psychological theories and research have provided several concepts which are essential to an understanding of determinants of divergent behaviours. Therefore, concepts from the Theory of Reasoned Action (Fishbein \& Ajzen, 1975), the Theory of Planned Behaviour (Ajzen \& Madden, 1986) and Bandura's concept of self-efficacy and modeling (Bandura, 1986) are presented. In order to gain more insight into determinants of specific health-protective behaviours, various social psychological models of health behaviours has been developed recantly, for example, the Health Belief Model (Rosenstock, 1974; Janz \& Becker, 1984), Weinstein's concept of unrealistic optimism (Weinstein, 1980) and the Protection Motivation Theory (Rogers, 1983). Each of these 
theories assume that the anticipation of a negative health outcome and the desire to avoid this outcome or reduce its impact, creates motivation for protection.

An extensive review of research on determinants (different concepts from the above theories) of the intention to STD-preventive behaviours (e.g. Intentions to use condoms) on the one hand and determinants of intentions to early STD detection behaviours on the other, is described in paragraphs 1.4.3 and 1.4.4. It starts with a review of research findings on determinants of intended STD-preventive behaviours. Selection and ordering of variables takes place according to the Predisposing. Enabling and Reinforcing factors, as mentioned in the PRECEDE-PROCEED model. Consecutively, determinants of early STD detection behaviours are discussed and are also classified under Predisposing, Enabling and Reinforcing factors. On the basis of these research findings a central research question is formed in the last part of chapter 1. In order to answer this central question we conducted several studies among different groups of respondents. Chapter 1 concludes with a rough description of the study population and the methods used in our studies. For a detailed description of the methods used in the different studies, we refer the reader to parts II and III of this thesis.

Chapter 2, a general discussion, starts with the presentation of major findings about determinants of behaviours aimed at primary prevention of STDs arnong adolescents and persons asking for an HIV test. In \$ 2.1.2 major findings of the studies on determinants of early detection behaviours are discussed. Attention will be paid to determinants of STD self-examination, periodic clinical examination and prompt help-seeking behaviour. The organisation of STD health care may also contribute to the early detection of STDs. Therefore, the most essential points of special service attributes in STD-health care are presented in the last part of $\$ 2.1$. In $\$ 2.2$. some methodological issues and limitations of our research are discussed, namely: the generalisability of the studies conducted, the problem of causality, self-report measures and the complexity of condom use. Chapter 2 ends with a discussion and recommendations to the following matters: (1) STD prevention activities, such as STD education and counselling; (2) interventions aimed at early detection of STDs; (3) patient care, such as the significance of service attributes in STD health care in the Netherlands.

PART II deals with the studies on STD-preventive behaviours among adolescents and among persons with an HIV test request. Three different studies among these two sanples are discussed. It should be mentioned here that these studies have been published in different journals and because of this there is an overlap between the introductions and methods of the separate presentations. Central attention is given on potential determinants of STD-preventive behaviour, namely, knowledge of STD transmission, attitudinal aspects of STD-preventive behaviour, social influence and self-efficacy expectations.

Chapter 3 presents the results of a study among heterosexuals with an HIV test request on determinants of the intention to use condoms during sexual intercourse with different partners. Subsequently, Chapter 4 describes some determinants of intended condom use with a casual partner arnong adolesents. Finally, Chapter 5 discusses the role of beliefs, evaluations and previous STD-preventive behaviours in the intention to STD-preventive behaviour among adolescents. 
PART III presents the studies on behaviours related to early detection of STDs, namely: STD self-exarnination, periodic clinical examination and prompt help-seeking behaviour among persons seeking an STD examination. In addition, the polential significance of specific service attributes in STD health care are discussed, based on a study among persons seeking an STD examination and a study among adolescents.

Chapter 6 deals with the determinants of two early STD detection behaviours: STD selfexamination and perlodic clinical STD examination, respectively. It describes the contribution of various determinants in predicting intentions to perform early STD detection behaviour, euch as some components of the Health Belief Model, previous STD detection behaviours and evaluations of early STD detection behaviours. Chapter 7 concentrates on determinants of delay-behaviour in persons with STD-related complaints. With regard to potential determinants of patients' delay the following items are included in the study: some components of the Health Belief Model, beliefs about the necessity of an STD examination, the attribution of symptoms to sexual behaviour and the significance of service attributes in STD health care.

Chapter 8 deals with the importance of an overall STD health care delivery system. The contribution of several service attributes (characteristics of health care facilities) in the choice of special STD health care facilities among persons seeking an STD examination is investigated. In addition to the potential influence of sixteen service attributes, ltems about shame and taboo on STDs are included in this study.

Finally, Chapter 9 presents the results of the investigation into the significance of service attributes in STD health care as perceived by adolescents. The contribution of sixteen service attributes in the explanation of choice of specific health care providers is assessed here.

Again, these chapters have been published (or are submitted for publication) in different journals and because of this the reader should consider that some matters will already have been presented before. 


\section{PART I}

\section{OVERVIEW}





\section{PLANNING hEALTH PROMOTION IN STDa: THE PRECEDE-PROCEED MODEL}

Health promotion may be described as a combination of educational and environmental support for actions and conditions of living conducive to health (Green \& Kreuter, 1991). Combination refers to the necessity of matching the multiple determinants of health with multiple interventions or sources of support. Educational refers to health education, defined as any combination of learning experience designed to facilitate voluntary actions conducive to health. Environmental refers to the social, political, economic, organisational, policies and regulatory circumstances bearing on the behaviour or more directly on health.

The PRECEDE-PROCEED health promotion planning framework (see Figure 1) developed by Green and Kreuter (1991) directs initial attention to desired outcomes. Whether the health promotion programme operates at the primary (hygiene and health enhancernent), secondary (early detection) or lertiary (therapeutic) stage of prevention, it may be accurately seen as an intervention whose purpose it is to short-circuit illness or enhance the quality of life by changing or developing health-related behaviour and living conditions (Green \& Kreuter, 1991).

In the PRECEDE-PROCEED model the main focus is on the importance of comprehensive planning. As can be seen in Figure 1 the model distinguishes nine basic phases. The first phase, the phase of social diagnosis, identifies factors which affect the quality of life in a certain population, namely more general problems (e.g. poverty, crime, discrimination) and health problems (e.g. specific diseases in a population). An analysis is usually made of data indicating the severity of the problems and the human and economic costs.

The task of the second phase, the phase of epidemiological diagnosis, is to describe a specific health problem in terms of its volume. The incidence and the prevalence of a specific disease in a given target population are central concepts in the first part of the epidemiological diagnosis. Identification of known risk factors that contribute to the health problem takes place in the second part of phase 2. More specifically, particular attention should be directed to identifying existing data on who is mostly affected (e.g. age, gender, race, residence).

A behavioural diagnosis is made in phase 3 and consists of describing specific modifiable behavioural causes of the health problem in question. The environmental diagnosis lists modifiable non-behavioural causes of the specific health problem, such as environmental and technological factors (e.g. aspects of medical care, health care facilities). 


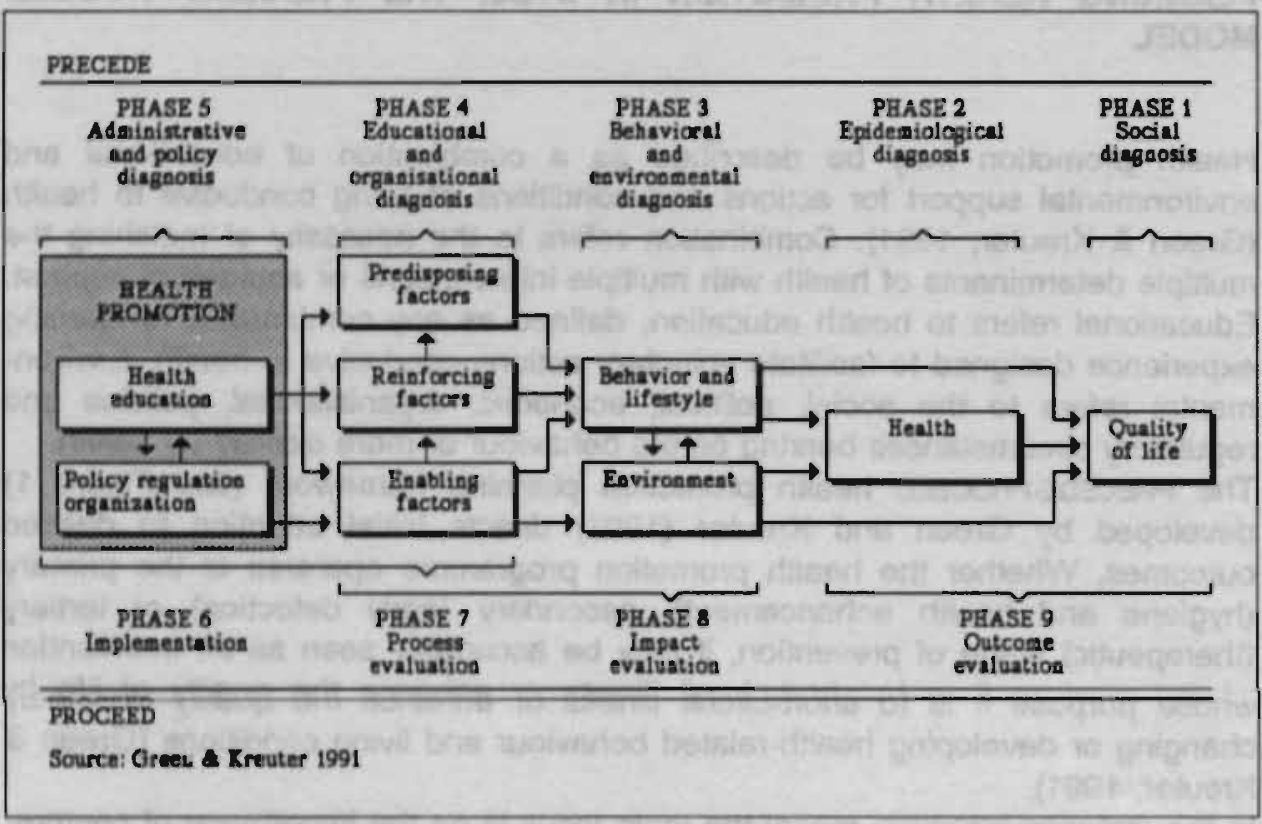

FIGURE 1. PRECEDE-PROCEED MODEL.

In the fourth phase, the phase of PRECEDE, determinants of identified behavioural risk factors are grouped according to an educational diagnosis likely to be employed in a health promotion programme. According to the model these determinants can be separated into three factors: Predisposing, Enabling and Reinforcing. Knowing how these factors work within a certain target group in a given context, one is able to plan the content of a health education programme.

Next, phase $\mathbf{5}$ deals with the assessment of organisational and administrative capabilities and resources for the development and implementation of a programme. Finally, phase 6 (implementation), phase 7 (process evaluation), phase 8 (impact evaluation) and phase 9 (outcorne evaluation) provide additional steps for developing policy and for initiating the implementation and evaluation process. The evaluation process, however, is considered to be an integral and continuous part of working within the entire framework.

The model can be used as a framework for the planning and evaluation of health promotion activities. Successful application depends; on the users' ability to utilise and integrate several relevant theories and empirical findings and to define the variables in each category of the planning model.

Fesearch on health promotion planning showed that the effectiveness of health education programmes is related to the quality of the planning process: (Mullen, Green \& Persinger, 1985; Green \& Kreuter, 1991; Kok \& De Vries, 1989; 
Jonkers, Liedekerken, De Haes, Kok \& Saan, 1988). So, in order to develop effective interventions for specific health problems, passing through such a planning process is very much advisable.

This thesis shows the process of determining the content of health promotion activities in the field of Sexually Transmitted Diseases.

The different diagnostic phases in the PRECEDE-PAOCEED model are mainly directed to factors (behavioural) that are changeable and therefore need interventions. By adopting the aforementioned framework for assessing varied needs and demands of interventions on STD prevention, one is able to take the complex array of influences into account in which STDs occur. Because of the ultimate goal of this thesis, gaining more insight into the determinants of STD. related health behaviours among specific target groups, it ends with an educational diagnosis, the fourth phase of the planning model and with recommendations to interventions. In 1987, the year we started the first study in the field of STDs, no such extensive health promotion programme was available. Because of that, we applied the PRECEDE-PROCEED model to STDs after we carried out our research. In addition, the description of the social, epidemiological, behavioural and educational diagnosis has been updated with results found after the research had taken place. Due to the ultimate goal of the different studies (see parts II and III), gaining more insight into determinants of primary prevention and early detection of STDs, central attention has been paid here to the educational diagnosis.

\subsection{Social diagnosis}

\subsubsection{Identifying STDs as an urgent health problem}

One of the first steps in planning health promotion activities is to establish why STDs are important. Morbidity and mortality data traditionally determine the urgency with which medical and public health problems are addressed. In the general outline we already noted that the prevalence of STDs has reached a disturbing level in many countries. When compared with other notifiable diseases, STDs are the most frequent occurring infectious diseases in the Netherlands. They can pass without visible symptoms, they can be transmitted perinately and in most of the cases early treatment is possible. Yet, many STDs are important not only for their infection itself, but also for the complications and their sequelae, particularly those which affect women. Complications, as mentioned by De Schrijver and Meheus (1990) include (1) adverse outcomes of pregnancy for mothers (post partum Salpingitis) and newborns (fetal death, prematurity and low birth weight), (2) infections in newborns and infants (iike 
opthalmia neonatorum and chlamydial pneumonia), (3) seguelae of Pelvic Inflammatory Diseases (PID), such as: infertility and ectopic pregnancy, (4) sequelae of urethritis and epidydimitis, such as: urethral stricture and male Infertility, and (5) cancers of the cervix, vulva, penis and anus associated with Human Papilloma Virus types 16 and 18. Moreover, infection with Human Immunodeficiency Virus (HIV), which ultimately leads to Acquired Immune Deficiency Syndrome (AIDS), has an enormous impact on the quality of life. Although there are parallels with patients suffering from other life-threatening diseases, the practical and emotional problems of AIDS are likely to be more complicated for people with AIDS (George, 1989).

Limited resources and current cornpeting health care needs, however, force the consideration of economic consequences as an additional criterion for determining the relative urgency of a problem. Treatment of persons with STDs is easier and cheaper at an initial stage of the disease than when complications are present. On average, a consultation for diagnosis and treatment of Sexually Transmitted Diseases (including prescribed drugs and overhead costs) costs about 500 guilders in the Netherlands (SOA-Stichting, 1986). The total direct costs of the medical treatment on complications of Chlamydia trachomatis Infections are estimated to be 27 million guilders (without the indirect costs) in 1987 in the Netherlands (Severijnen, Steyerberg \& Huisman, 1992). A total of 765 women diagnosed with infertility caused by STD pathogens were hospitalised in 1987. Direct costs (without laboratory diagnostics and medicine treatment) were calculated to be $\mathbf{3 . 9}$ million or $\mathbf{5 0 0 0}$ guilders per woman (Severijnen \& Huisman, 1992). Lastly, the costs of medical treatment of one person with AIDS are estimated to be minimally ^ $\mathrm{U} 70,000$ in the United States (Scitovsky \& Over, 1988) and about 112.000 guilders in the Netherlands (Reinking, Postma, Albers, Van den Boom \& Jager, 1992).

Another way of establishing the enduring importance of STDs is to look at the influence of social and cultural aspects of sexuality on the occurrence of STDs. Demographic, sociological and behavioural changes occurring in the Dutch population contribute to the growing importance of these diseases and will continue to do so in the near future. The number of young adolescents having sexual intercourse is increasing, the population is shifting from traditional rural to urban areas. Family and community ties are changing, consequently there is diminished social control over behaviour. From this finding we may conclude that more people are at risk of contracting STDs than ever before and these trends seem likely to continue.

The above findings suggest that not only the costs of complications related to untreated STDs are important, but it is also apparent that the quality of life is affectecl through such complications as mentioned by De Schrijver and Meheus 
(1990). In this respect STDs and their complications have serious consequences not only for the individuals and their families but also for the community and, therefore, STDs rank as formidable health problems. In the absence of adequate cure of most viral STDs, prevention of Sexually Transmitted Diseases and prevention of developing STD-related complications is needed both to reduce human suffering and to cut rising costs.

\subsection{Epldemlologlcal diagnosis}

In order to assess the importance of a specific health problem such as STD3, and to set priorities for STD-related interventions a second step in planning STD health promotion strategies is necessary in which morbidity data of major STDs (\$ 1.2.2) and the incidence of complications are examined in detail (\$ 1.2.3). An underlying principle of disease epidemiology is that, for example, STD3 and STD-related complications are not evenly distributed throughout the population. As a result some groups within the population are of greater importance 10 prevention and control than others. Therefore, in the second part of the epidemiological diagnosis nonbehavioural and nonmodifiable risk factors for contracting STDs and those for developing complications are described (\$ 1.2.4).

\subsubsection{Classification and characteristics of major STDs}

STDs can be seen as syndromes (clinical manifestations) or can be classified by their STD pathogens. Over twenty pathogens are known to be transmitted by sexual contact (see Table 1).

The nature of the predominant symptoms and complaints can be listed as follows: pain, painful urination, abnormal discharge, itching, burning or other irritation, unpleasant smell and other visible abnormalities. The clinical manife. station or spectrum of STDs is broad, including symptomatic, asymptomatic and complicated infections of several anatomic sites. Wigersma (1990) distinguishes the following syndromes: urethritis, epididymitis, vulvo-vaginitis, cervicitis, Pelvic Inflammatory Disease (PID), proctitis, genital/anal ulcera, genital/anal warts, intestinal infections, skin lesions and systemic diseases.

While medicines are available for most bacterial STDs, adequate medical treatment has to be found for several (new) viral STDs. In addition, the persistent character of some vira! infections with asymptomatic viral excretion also underlines the importance of primary prevention of STDs. With regard to bacterial STDs, the occurrence of adaptive changes in STD pathogens (Penicilfnase Producing Neisseria Gonorrhea) should also be mentioned here. Lastly, infection with HIV must be mentioned as a lethal disease in the range of Sexually Transmitted Diseases. 
TABLE 1. CLASSIFICATION OF SEXUALLY TRANSMITTED DISEASE AGENTS

1. Bectorlal agents

Nolseerla gonorrhose

Clamydia trachomatis

Treponema pallidum

Haemophlilus ducreyl

Mycoplasma hominis

Ureaplasma urealyticum

Calynnalobacterium granulomatis

Shigella epp.

Campylobacter epp.

Group B streptococcus

Bacterial vaginosis-associatod organisms

3. Protozoal agenta

Entamcoba histolytica

Giardia lambia

Trichornonas vaginalis

Phthirus pubis

Sacroptes scabiel
2. Vral agente

Herpes Simplex Virus type 1 and 2

Cytomegalovirus

Hepatitis B virus

Human Papiloma Virus

Molluscum Contagioeum Virus

Human Immunodeficiency Virus

Source: Do Schrijver \& Mohous, 1990

\subsubsection{Trends of major STDs}

In this paragraph we will present a rough picture of the occurrence of STDs. Attention is only paid to the most important STDs: gonorrhea, syphilis, Chlamydia trachomatis and infection with HIV.

Unfortunately, only a few countries have reporting systems that permit accurate estimations of the true inciderice of STDs. Since 1976, gonorrhea and primary and secondary syphilis are notifiable diseases in the Netherlands and have been registered by the Chief Medical officer of the Ministry Welfare, Public Health and Culture (GHI). Because of a considerable amount of underreporting (estimations range from $60 \% 1095 \%$ in different regions), the national notification data for gonorrhea and syphilis only permit estimations of true incidence of these STDs (Miltenburg. Paalman \& Reus, 1985). Therefore, interpretations of trends need to be undertaken cautiously. Table 2 shows the annual incidence of reported gonorrhea cases and of primary and secondary syphilis.

Table 2 indicates that the reported incidence of gonorrhea in the Netherlands reached a peak in 1981, showed a stable pattern between 1981 - 1984 and has declined dramatically since 1985. 
TABLE 2. THE NUMBER OF NOTIFIED CASES (MEN AND WOMEN) OF GONORRHEA AND SYPHILIS IN THE NETHERLANDS IN THE PERIOD 1976 - 1992

\begin{tabular}{lrr}
\hline YEAF & GONORRHEA & SYPHIL.S \\
\hline 1976 & 7,334 & 692 \\
1977 & 9,005 & 767 \\
1978 & 9,613 & 999 \\
1979 & 11,051 & 1,196 \\
1980 & 12,264 & 1,169 \\
1981 & 14,855 & 999 \\
1982 & 13,425 & 1,027 \\
1983 & 14,400 & 924 \\
1984 & 13,199 & 913 \\
1985 & 12,451 & 584 \\
1986 & 9,841 & 576 \\
1987 & 5,636 & 388 \\
1988 & 3,647 & 394 \\
1989 & 3,024 & 539 \\
1990 & 3,666 & 499 \\
1991 & 2,896 & 253 \\
1992 & 2,397 & 189 \\
\hline
\end{tabular}

Source: Chiel medical officer of the Dutch Ministry of Welfare, Health and Culture (GHI)

In imitation of gonorrhea, the incidence of syphilis remained fairly constant during 1981 - 1984, followed by a decline in 1985 until 1987, was stable between 1987 - 1989 and has declined further since then. With regard to gonoccoccal resistance to penicilline it has been shown that after a decrease to 400 cases during the period 1986-1988, an increase to 1100 cases had been reported in 1990, followed by a decline to about 500 cases in 1991 Nan de Laar, Schellekens \& Van Klingeren, 1993).

Until now Chlamydia infections and other STDs have not been registered, so the exact incidence and trends over times are unknown. Yet, some studies among specific population groups show that genital infections caused by Chlamydia trachomatis exceed the incidence of gonorrhea in the Netherlands (Severijnen \& Huisman, 1992). Based on a variety of data from STD clinics and other prevalence studies together with the gonorrhea notification data of the GHI, about 90,000 individuals were estimated to have been infected with Chlamydia trachomatis in the Netherlands in 1987 (Severijnen, 1993). De Schrijver and Meheus (1990) also suggest that infections with Chlamydia trachomatis exceed the incidence of gonorrhea

The estimated cumulative incidence of HIV infections in the Netherlands as of January 1989 is between 9,000-12,000 (Jager, Poos, Houweling, Postema \& Coutinho, 1990) and between 7,500-9,000 in mid 1990 (Nan de Water, Wiggers, 
Bijleveld \& Berkane, 1990). Yet, these estimations are based upon mathematical models. Until now, HIV infections and AIDS are no obligatory notifiable diseases in the Netherlands. Anyhow some trends may be distinguished among different risk groups. Yet, Houweling and Jager (1993) concluded in their review of these studies that significant differences on HIV-seroprevalence are found (between the investigations) within risk groups, such as: men with homosexual contacts and Intravenous Drugusers (IVDU). Lastly, about $13 \%$ of the 1,250 hemophilic patients are HIV-seropositive because they became HIV infected through bloodproducts (Roosendaal, Smit, Verekamp, Brocker, Van Dijck, Suurmeyer \& Briet, 1988).

Physicians notify voluntarily and anonymously ADS diagnosis to the GHI. The cumulative incidence of ADS at Juni 1993 amounted to 2678 diagnosed ADS cases (see also Figure 2, \$ 1.2.4) (GHI, 1993). For a description of the estimated HIV seroprevalence and ADS among specific groups the reader is referred to $\$ 1.3 .1$.

\subsubsection{The Incidence of major complications of STDs}

Owing to the fact that the most important complications of STDs (such as PID) are found in women, this paragraph mainly concentrates on PID in women. Pelvic Inflammatory Disease, infections of the upper genital tract, is the major cause of infertility, ectopic pregnancy and chronic pelvic pain in women (Cates, Rolts \& Aral, 1990). The majority of proven cases of PID are caused by Chlamydia trachomatis and Neiserria gonorrhea Westrom, Svensson, Wolner-Hansen \& Mardh, 1982; Pavoonen, Geisala \& Heinonen, 1987). Based on literature findings, Severijnen and Huisman (1992) assume that two-thirds of all PID cases are caused by Chlamydia trachomatis in the Netheriands. Owing to minor and vague complaints many PID cases are not recognized or are recognized until the acule phase has taken place. It is also assumed that asymptomatic PID occurs as onen as symptomatic PID Wolner-Hansen, Kiviat \& Holmes, 1990). Yet, the mechanisms and likelihood of the spread of these STDs from the lower to the upper genital tract are, however, still poorly defined (Rice \& Schachter, 1991).

Registration of the Amsterdam Sentinel of General Practitioners shows that the incidence of PID declined from 50 to 24 in 10,000 women in the period 1983 . 1990 (Coutinho, Rijisdijk, Van den Hoek \& Leentvaart-Kuiper, 1992). This decline did not take place among females younger than 20. Based on a nationally representative sample, one study (Aral, Mosher \& Cates, 1985) reported that one in seven American women of reproductive age had had PID. one in 10 had received ambulatory care and one in 25 , had been hospitalised for PID in 1982. Updated estimates for 1988 of the cumulative incidence of self- 
reported PID derived, showed a lower cumulative incidence in 1988 in all demographic subgroups (Aral, Mosher \& Cates, 1991). In addition, Rolfs, Galaid and Zaidi (1990) also reported that, while the absolute number of cases of PID has fallen recently, the percentage of women with PID who are younger than 24 and white has been increasing.

The incidence of infertility after first, second and third episodes of PID in women has been calculated to be $8 \%, 22 \%$ and $40 \%$ respectively (Westrom, Joesoef, Reynolds, Hagdu \& Thompson, 1992). With regard to intertility in the Netherlands, it was found that in about $25 \%-30 \%$ of the women attending a programme for In Vitro Fertilization in one hospital, the infertility could be attributed to the complications of Chlamydia trachomatis infection (Peeters, Bots, Merkus \& Prak, 1985).

Complications of STDs in male patients are sequelae of urethritis and epididymitis, such as urthral stricture and male infertility. Little information is available on the occurrence of male infertility related to STD pathogens.

\subsubsection{Risk factors for contracting STDs and for developing complications}

Although the PRECEDE-PROCEED model is mainly directed to factors that are changeable, unchangeable factors such as age, gender, race or ethinicity, sexual orientation and rural versus urban residence should also be considered. It is apparent that it are not the unchangeable factors, but the specific; behaviours associated with these factors that are actually the risk factors for contracting an STD. These factors do not lend themselves to interventions, but they must be taken into account to keep a perspective on the multiple cleterminants of STDs and to identify high-risk populations. Until recently only a few researchers in the field of STDs have differentiated between risk factors for initial infection and risk factors for the development of STD-related complications. In this paragraph we will only give a rough presentation of these nonmodifiable risk factors.

Nonmodifiable risk factors:

A recent study showed that reported cases of syphilis and gonorrhea and other STDs in the Netherlands tend to be concentrated in inner city areas, namely Amsterdam and Rotterdam, and are found more frequently in homosexual men, persons aged 25-29 and 30-34, and in female prostitutes Nan de Laar, Pickering, Van den Hoek, Van Griensven, Coutinho \& Van de Water, 1990). In addition, data from the STD clinics in Amsterdam showed that $50 \%$ of women with gonorrhea are prostitutes, $35 \%$ of the men acquired gonorrhea by sexual contact with a prostitute and $26 \%$ of the men are homosexual Nan de Laar et 
al., 1990). Almost $40 \%$ of the attenders of STD clinics has already had a gonorrhea infection before. These recurrent attenders may be defined as 'core groups' in the spread of gonorrhea. The term core groups of disease transmitters suggests that a subgroup of infected persons is responsible for the transmission of STDs in the community. Other groups are also recognised as possible risk groups. A recent study showed that gonorrhea figures are probably overrepresented in ethnic minority groups, mainly men of Turkish origin Nan de Laar, Sleutjes, Postema \& Van de Water, 1992).

Little is known about risk factors for contracting Chlamydia infections. Van de Laar et al. (1990) showed that Chlamydia trachomatis occurs relatively less often in men with homosexual contacts and ethnic minority groups than in heterosexuals and native Dutch persons. Similar to gonorrhea infection, Chlamydia trachomatis has a peak incidence in the 20-29 age brackets (SOA Stichting, 1990). With regard to the HIV-epidemic (GHI, 1993) it is said that AIDS cases are concentrated among men with homosexual contacts and Intravenous Drug Users (IVDU) (see Figure 2).

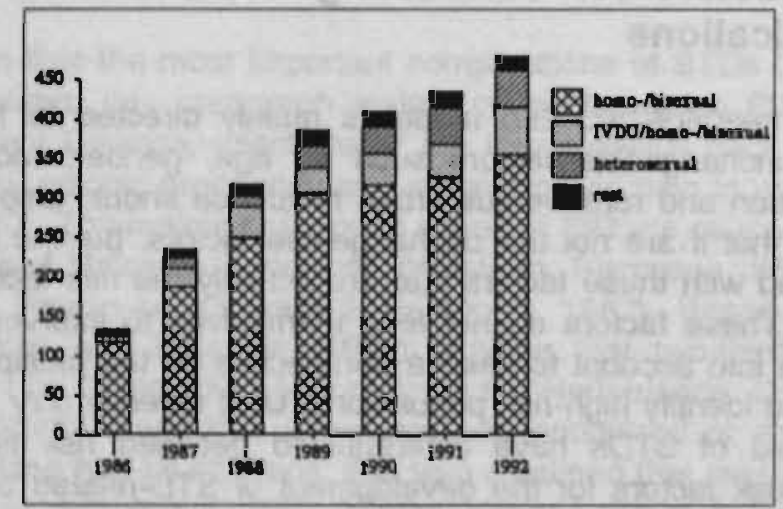

Souree GHI, 1993

FIGUAE 2. DIAGNOSED AIDS CASES BY RISK GROUP TO YEAR OF DIAGNOSIS

From 1982 to 1985 all diagnosed AIDS patients were men with homosexual contacts and at this moment three-quarters of the diagnosed AIDS patients still belong to this category of individuals. About $7 \%$ of the diagnosed AIDS cases are women.

The share of the second most important group of AIDS patients, the Intravenous Drug Users, has increased to $10 \%$ in recent years. More than half of the AIDS 
cases have been diagnosed in Amsterdam (GHI, 1993). As of June 1993, $10 \%$ of the diagnosed AIDS cases were transmitted heterosexually.

Apart from these nonmodifiable risk factors for contracting STDs it has been shown that age is a risk factor for the development of PID. Teenagers have a particular high rate of PID, especially when corrected for sexual activity (Washington, Aral, Wolner-Hansen, Grimes \& Holmes, 1991). These authors Washington et al., 1991) concluded in their review on risk for PID that such factors as education, employment and income are all risk markers (confounders, have an indirect effect) instead of risk factors.

From the above medical and epidemiological findings we may conclude that STDs, their complications and their late sequelae are major public health problems with considerable emotional and economic consequences. The findings on the incidence of specific STDs also suggest that the following risk groups for contracting STDs (priority groups), including HIV infection, can be distinguished: men with homosexual contacts, adolescents and young adults (with multiple sex partners), prostitutes and their clients, and ethnic minority groups.

\subsection{Behavioural and environmental diagnosis}

Apart from the epidemiological diagnosis, the third phase of the PRECEDEPROCEED model consists of exploring modifiable behavioural and environmental factors related to STDs. In this review a distinction has been made between behavioural factors which increase the risk of exposure (behavioural risk factors) on the one hand and those behaviours which decrease the risk of STD infection (STD-preventive behaviours) on the other (see \$ 1.3.1). After the presentation of more general behavioural factors a detailed description is presented per risk group (priority group) of behavioural risk factors and preventive health behaviours. Subsequently, behavioural risk factors for the development of complications and preventive behaviours aimed at early STD detection are analysed in $\$$ 1.3.2. Lastly, in \$ 1.3.3 environmental factors influencing the occurence of STDs and STD-related complications are presented.

\subsubsection{Assessment of behavioural factors pertaining to contrac- ting STDs}

\section{Assessment of behavloural risk factors}

STDs are transmitted primarily through the exchange of bodily fluids of infected sexual partners. HIV infection, Hepatitis B virus, and syphilis are also transmitted through the use of contaminated equipment by intravenous drugusers 
(needle sharing) and infected bloodproducts. In this paragraph, however, we will only pay attention to the sexual behaviour which will lead to the transmission of STDs, including HIV.

The importance of particular sexual behaviours in STD transmission has been highlighted by the AIDS epidernic. It is generally assumed that the most important STD and HIV-related problem behaviours are:

- unprotected analvaginal sexual intercourse with a new/casual partner, with multiple partners and with a nonmonogamous permanent partner;

- unprotected oral sex with a new/casual partner, with multiple partners and with a nonmonogamous permanent partner.

Apart from unprotected sexual intercourse with (infected) individuals belonging to high risk groups, multiple partners and a history of STDs have been found to be major risk factors for heterosexual transmission of HIV (CDC, 1988; Greenblatt, Lukehart \& Plurnmer, 1988). That the number of sexual partners within a specific time period is an important risk factor for contracting gonorrhea, Chlamydia trachomatis, genital herpes and Human Papilloma Virus infections is underlined by several studies (D'Costa, Plummer, Bowmer, Franser, Pilot \& Ronald, 1985; Handsfield, Jasman, Roberts, Hanson, Kothenbeutal \& Stamm, 1986; Schachter, Stoner \& Moncada, 1983). It is important to note, however, that if sexual techniques are used which do not transfer bodily fluids, the number of sexual partners is of no consequence.

\section{Assessment of STD-preventlve behaviour}

STD-preventive behaviours specifically aimed at primary prevention of STDs may range from total abstinence, having a mutual monogamous relationship, waiting longer to become sexually active with someone, performing safe sex techniques (e.g. massage, masturbation), avoiding high-risk sexual behaviour to consistent condom use. From a pragmatic perspective the limitations of total abstinence and fidelity as a universal control strategy are obvious.

In general, condom use is accepted as the most desirable and effective way of preventing transmission of STDs. Clinical studies have suggested, however, that condoms are effective in preventing the transmission of some, but not all STDs (Stone, Grimms \& Magder, 1986; Wellings, 1986). Recent laboratory studies tend to support these clinical findings. Synthetic (latex) condoms are likely to be effective in the transmission of viral pathogens, including HIV, while natural condoms appear to be effective only in preventing the transmission of the large viruses, (Munik, 1989). Lisking, Wharton and Blackburn (1992) also stated that latex condoms have proved to be effective against nearly all STDs, whereas condoms made of sheep intestine (skins) may allow passage of some sexually transmitted pathogens and are therefore not approved for disease prevention. 
HIgh-rlak groupe, behavioural risk factore and STD-preventlve behaviour In \$ 1.2.4 five risk groups (priority groups) for contracting STDs have been distinguished. Hereafter, these risk groups will be presented separately. Per risk group the following items are discussed: the estimated number of individuals belonging to the risk group, the estimated incidence of gonorrhea, syphylis and HIV infection, specific sexual techniques that place the individuals at risk of contracting STDs and lastly, in order to prevent STDs, the frequency of using condoms and other safe sex behaviours.

Since about $60 \%$ of the reported gonorrhea cases are diagnosed in the STD clinics in Amsterdam and Rotterdam (GHI, 1992) several sociodemographic characteristics per risk group are described on the basis of these data.

\section{Men with homosexual or blsexual contacts}

It has been estimated that $4 \%$ of the Dutch population have homosexual contacts; a total of 125,000 men aged 20-45 Nan Zessen \& Sandfort, 1990). About 25,000 homosexual men live in Amsterdam (Houweling, Jager, Coutinho, Bijkerk \& Ruilenberg, 1987). It is also estimated that there are about $10,000-15,000$ married men with homosexual contacts (Deenen \& Van Naerssen, 1988; Haverkos, Bukoski \& Amsel, 1989).

Van de Laar et al. (1990) showed that the incidence of gonorrhea among men with homosexual contacts diagnosed in STD clinics in Amsterdam declined from one-third to $12 \%$ ( $=131$ cases) of all men with gonorrhea during the period 1981-1987. Since 1988, however, an increase in the number of diagnosed gonorrhea cases among homosexual men has been observed at these STD clinics in Amsterdam (Van den Hoek, Van Haastrecht, Henquet \& Coutinho, 1993). In 1991 the total number of gonorrhea cases among homosexual men reached the level of 1986, with 277 cases of gonorrhea (138 with anal gonorrhea) being diagnosed among men with homosexual comtacts (Van den Hoek et al., 1993). Similar figures were found in Rotterdam; of the 285 diagnosed gonorrhea cases in Rotterdam in the period of $1990-1991,18 \%$ concerns homosexual men. Among men with homosexual contacts who asked for an HIV test at the STD clinics in Amsterdam, 10\% were HIV-seropositive and of 324 homosexual men who were tested anonymously, 22\% were HIV-seropositive Nan den Hoek, Fennema, Van Haastrecht, Van Doornum, Henquet \& Coutinho, 1992). In addition, after a decline in the number of HIV-serocomversions among homosexual men in the Amsterdam cohort study (43 in 1985 to 4 cases in 1989), an increase in the number of seroconversions was observed in 1990 Nan den Hoek, Van Griensven \& Countinho, 1990). In general, these data reveal that after a significant decline in the occurrence of gonorrhea and syphilis this trend was reversed as the number of cases of gonorrhea among homosexual men started to increase again. 
There is a growing body of information involving specific sexual techniques in the emergence of STDs common among men with homosexual contacts. In a study among gay-men, Altman and Ostrow (1980) observed that specific sexual techniques susceptible to contracting hepatitis B, syphilis and gonorrhea can be distinguished into (1) receptive anogenital intercourse; (2) oro-anal intercourse; (3) insertive anogenital intercourse; (4) rectal douching in association with receptive anogenital intercourse, and (5) receptive manual-anal intercourse. In addition, cohort studies of homosexual men in developed countries yielded important data about behavioural risk factors for HIV infection. These studies have uniformly shown that large numbers of partners combined with a high percentage of sexual encounters, such as receptive anal intercourse unprotected by condoms, substantially increase a homosexual man's risk of becoming infected with HIV (Darrow, 1987; McCusker, Zapka, Stoddard \& Mayer, 1989a; Van Griensven, Tielman \& Goudsmit, 1987; Kuiken, Van Griensven, De Vroome \& Coutinho, 1990). In line with Holmberg. Stewart and Russell (1988) the existence of Herpes infection has proven to be a second major risk factor for contracting HIV-infection (Kuiken et al., 1990). From this finding it might be concluded that the number of sexual partners as a risk factor for HIV infection may depend on the type of sexual technique used. Avoidance of unprotected anal intercourse has consistently reduced risk of infection with HIV in other cohort studies (Darrow, 1987).

When unprotected anal intercourse cannot be avoided, the safe and proper use of condoms (yet, the failure rate is relatively high) may be effective in preventing STD and HIV transmission. It may be concluded from several studies that the regular use of condoms among Dutch homosexual men is not a long-term practice (Kuiken et al., 1990; Van den Hoek et al., 1993). A recent study (De Wit, Van Griensven, Sandfort \& Coutinho, 1993) among the Amsterdam cohort showed a significant increase $(13 \%$ to $24 \%)$ of unprotected anogenital intercourse with casual partners in the period of 1990-1991. These data also revealed that the percentage of men with unprotected insertive and/or receptive sexual intercourse with a regular partner stayed about the same during this period (25\%).

\section{Adolescents and young adults}

As of 1 Januari 1993 about $21 / 4$ million adolescents and young adults (age 1524 years) live in the Netherlands. The notification data of the social nurses working at Municipal Health Services (MHS) and STD clinics in the Netherlands show that $30 \%$ of all STD diagnoses occur within the 15.19 and 20-24 age brackets $(\mathrm{GHI}, 1992)$. In addition, the average age pattern in reported gonorrhea cases shows a peak for males in the 25-29 and 30-34 age brackets. The peak for fernales has moved from the 20-24 to 25-29 and 30-34 age brackets since 
1980 (Van de Laar et al., 1990). Data on the reported syphilis cases showed that about $30 \%$ of the syphilis cases in wornen occur within the age brackets 15-19 and 20-24 years, while $10 \%$ of the men with a reported syphilis fall within these age categories (Schellekens, Schouls \& Van de Laar, 1993). In addition, data on the diagnosed AIDS cases reveal that about 60\% ( $N=1478)$ occur before the age of forty (GHI, 1993).

During adolescence many teenagers initiate sexual intercourse and more adolescents engage in initiation at an earlier age than their counterparts ten years ago (Cates \& Raugh, 1985). As a consequence they place themselves at risk for contracting STDs at earlier ages than before. A recent study on the sexual behaviour of Dutch adolescents also depicts a general trend of an increasing proportion of Dutch adolescents, who are sexually experienced (Nogels \& Van der Vliet, 1990). In general, about one-third of the adolescents aged sixteen and half of those aged 17-18 have had sexual intercourse. Apart from this they are more often sexually experienced than ten years ago and they have also switched more often from one sexual partner to a second partner. About $40 \%$ of the adolescents aged 12.19 have had two or more sexcual partners, while most of these relationships ended within three months (serial monogamous relationship).

In heterosexual contacts condoms can prevent both pregnancy and STDs. A study of Vogels and Van der Vliet $(1990)$ reveals that $60 \%$ of the adolescents used a condom during the first and $40 \%$ used a condom during the last sexual intercourse. Nevertheless, the most important motive to use condoms was protection against pregnancy; only $13 \%$ used the pill as well as a condom. That condom use is not a regular practice among adolescents is also supported by several other researchers (De Wit, Kok, Timmermans \& Wijnsma, 1990; Delii, 1988; Gieling \& Heuzer, 1988; Richard, Van der Pligt \& De Vries, 1991); more than half of the sexually active adolescents use condoms irregularly or they never use them.

\section{Prostitutes and thelr customers}

It is estimated that in the Netherlands 15,000 to 20,000 prostitutes, including them 1,300 males work in street, window and club prostitution, via escort services and in private situations (NCAB, 1990a; Boutellier, 1987). A.part from prostitutes of Dutch origin there is a group of highly mobile prostinutes from abroad who are difficult to reach and who are increasing in nurnber (Brussa, $1987 ;$ NCAB, 1990a). It is also estimated that almost $20 \%$ of Dutch men visit a prostitute at some point in their lives and $3 \%$ of them do this regularly (Boutellier, 1987).

The overall incidence of STDs and HIV infection among prostitutes in the Netherlands is unknown. Data from the surveillance system of the social nurses 
suggest that the number of women with syphilis has increased from 135 to 159 in the period 1986-1990. These figures also suggest that the share of female prostitutes has increased (23\% to $31 \%$ in 1990). In line with these findings the data from the STD clinics in Amsterdam show that about $50 \%$ of the diagnosed syphilis cases are related to prostitution (GGD, Amsterdam, 1990). Furthermore, Van de Laar et al. (1990) reported that $42 \%$ of the gonorrhea infections diagnosed among females at the Amsterdam STD clinics in 1987 occurred in those who claimed to be prostitutes. In addition, Van den Hoek and Van Haastrecht (1988) stated that HIV-infections are mainly observed in prostitutes who use drugs intravenously.

Recent studies on the sexual behaviour of prostitutes and their customers show high risk sexual behaviour among a relatively large number of the prostitutes and their clients. The number of sexual contacts for street prostitution is estimated at 4-5 a day, mostly protected sexual contacts in a four to five-day working week (De Graaf, Vanwesenbeeck, Van Zessen, Straver \& Visser, 1992). However, about 40 unprotected sexual encounters are also reported (Hooykaas, Van der Pligt, Van Doornum, Van der Linden \& Coutinho, 1989; Van Mens, 1989). Some researchers Nan Haastrecht, Van den Hoek \& Coutinho, 1990) noted a decline in the number of sexual partners of prostitutes attending an STD clinic. They also found that a small number $(9 \%)$ of these prostitutes had had receptive anal intercourse during the last six months, while about twothirds of the prostitutes stated they almost always use a condom during sexual intercourse (Nan Haastrecht et al., 1990). In another study among prostitutes (De Graat et al., 1992) it was found that $19 \%$ of the prostitutes and $25 \%$ of their customers do not use condoms consistently in commercial heterosexual contacts.

\section{Ethnic minority groups}

The exact number of persons with a non-Dutch cultural background living in the Netherlands is unknown.

A study into the share of the immigrant populations of Turkish, Moroccan, Surinamese and Antillian origin suggests that in the total STD population, the ethnic minority groups appear to be overrepresented (Van de Laar et al., 1992). Their share has increased from one-eighth to one-fith of the total number of STD cases. Caution, however, has to be taken in interpreting these results because the STD rates are based upon the registrations of social nurses working in MHSs and STD clinics. It may be that the ethnic minority groups have changed from seeking care at a general practitioner to STD clinics. Similarly, members of these groups who are not registered (illegal inhabitants) may have a preference for anonymous treatment facilities (STD clinics). Van Haastrecht et al. (1990) also found that Turkish men and immigrant prostitutes 
have shown an increase in STD examinations at the STD clinics as opposed to Dutch men and women, since 1987.

It is often assumed that these specific populations display higher rates of STDs because social control on sexual relationships are weak and promiscuity prevails. Differences in health behaviour (to detect and respond to symptorns, to seek prompt and effective medical treatinent, and to comply with directions for successful treatment) may also be important in explaining differences in STD infections. Research on the sexual behaviour and condom use of ethnic minority groups is rare in the Netherlands. One study (Van Haastrecht ef al., 1990) showed that about $82 \%$ of attenders of the Amsterdam STD clinics of Surinamese/Antillian origin never or irregularly used condoms in 1988 , while $71 \%$ of the persons with a non-Dutch cultural background (Turkish, Morroccan etc.) claimed to never or irregularly use condoms.

In summary, we conclude from these findings that a large part of the Dutch population is still at risk of contracting an STD. Within these specific risk groups a large part continously performs high-risk sexual behaviour and because of that they are important target groups for STD/HIV-related interventions.

\subsubsection{Assessment of behavioural factors pertaining to the development of complications}

\section{Assessment of behavloural risk factors}

As stated earlier, untreated STDs may cause serious complications, such as PID, especially in women. Several dimensions of sexual behaviour have been associated with an increased risk of PID: STD-history, age at first sexual intercourse, multiple sex partners, high frequency of sexual intercourse, and the rate of contracting new partners within a specific period of time Wolner-Hansen, Eschenbach \& Paavonen, 1990; Marchbanks, Lee \& Peterson, 1990; Lee; Rubin \& Borucki, 1988; Aral, et al., 1991). In addition, data from several studies suggest that women with PID, compared to women without PID, are more likely to have a history of vaginal douching after sexual intercourse, and of substances abuse (Wolner-Hansen et al., 1990). Yet, most of these studies fail to diflerentiate between the role of sexual behaviours as a risk factor for contracting lower genital tract infection and their role in the subsequent development of PID. Individuals who respond promptly and appropriately to symploms suggesting a chlamydial or gonococcal infection are presumed to be at lower risk for subsequently developing PID than those who delay in seeking medical care (Was. hington, Cates \& Wasserheit, 1991). It is also assumed (Washington et al., 1991) that individuals diagnosed with chlamydial or gonococcal cervical infection or PID who comply with their clinicians' therapy are likely to decrease their risk 
for developing subsequent complications. Yet, the relationship between patients' delay and compliance with therapy, and risk of developing PID has not been studied thoroughly.

\section{Assesement of preventive behaviours aimed at early detection of STD8}

Behaviours aimed at the prevention of PID involve keeping a lower genital tract infection from ascending to the upper genital tract. Health care behaviours which can reduce the risk of contracting complications after being infected with STDs include early consultation for diagnosis and treatment, compliance with therapy and follow up treatment for an infection, and patient referral Washington et al., 1991). Although compliance with therapy and patient referral (partner notification) are important behaviours related to reducing risk of complications and reinfection, only behaviours aimed at early consultation for diagnosis and treatment are discussed here.

Behaviours aimed at early consultation for diagnosis and treatment may include: STD self-examination (self-diagnosis) and monitoring, periodic clinical examination and prompt help-seeking behaviour. STD self-examination may involve inspection of one's genitals for abnormal discharge, unpleasant smell, itching. burning or other irritations, pain or painful urination, genital warts, ulcerations, and other visible abnormalities. Failure in STD self-examination and selfdiagnosis which should precede treatment, may also cause delay in seeking medical examination. Yet, as noted before, STD-related symptoms can be relatively mild or inconspicuous, and they might even be totally absent. To our knowledge no studies on the performance of STD self-examination have been carried out. Research on other self-examination behaviour such as Breast SelfExamination (BSE), has shown BSE to be efficient in discovering tumors at an earlier, more clinically treatable, stage (Tamburini, Massara, Bertario, Alberto \& Putio, 1981).

At present there are no accurate estimates either of the annual number of physician consultations or of the number of persons who do not seek medical examination for a suspected STD. Persons who do not seek medical examination immediately after noticing STD-related symptoms may transmit these communicable diseases in the community. There is evidence, however, that STD patients wait and see if symptoms persist, worsen or subside (CDC, 1980; Stadlander \& Kok, 1989). In his study on women's treatment decisions, Harrisson (1982) found that the reason why women did not seek medical treatment for genital symptoms was partially explained by classifying these symptoms as normal. When women were uncertain about symptoms, they stated that this uncertainty motivated them to seek medical assessment. Nevertheless, only $45 \%$ reported seeking medical treatrnent promptly. Data from the Centers for Disease Control $(\mathrm{CDC})$ in the United States indicated that men who have 
primary syphilis delayed seeking medical treatment on average twelve days after the initial appearance of their lesion. This delay behaviour varied with the educational background of the patient (CDC, 1980). Furthermore, one study reported that adolescents apparently showed longer delay behavior than older women (Spence, Adler \& McLellan, 1990). Orduna (1980) suggested that, on the basis of a study on gonorrhea transmission, if the duration of symptoms for each heterosexual patient was reduced by one day, the number off reported gonorrhea cases would decline by approximately eight percent.

Regular clinical check-ups may be seen as preventive behaviour aimed at the development of complications of STDs. Periodic clinical examination has also been suggested as an intervention to decrease the prevalence of STDs in prostitutes (Plummer \& Ngugi, 1990). Yet, no data are available on the effectiveness of this 'prevention strategy' for the development of complications in individuals with multiple partners.

From the above findings it is suggested that behavioural variables associalled with early diagnosis and treatment may involve STD self-examination, periodic clinical examination and prompt help-seeking behaviour after noticing STDrelated symptoms or after partner notification.

\subsubsection{Environmental factors influencing STDs and STD-related complications}

Modifiable environmental causes of STDs deal with the adequacy of medical care and the access to STD health care facilities. The attitudes towards STDs of the health providers may also contribute to the problem. For example, if the staff in an STD clinic interpret their role as a judgmental one, the patient may be deterred from attending during current or future infections. Washington et al. (1991) mentioned clinical training in STDs, detecting asymptomatic STDs, treating infections appropriately, counselling to reduce future risks and notifying all sex partners as effective means of minimising the risk of adverse consequences in patients and preventing the spread of STDs in the community. Unfortunately, no data are available on the effectiveness of these separate STD-controll activities on the development of complications.

\subsection{Educational and organisational diagnosis}

The fourth phase of the PRECEDE-PROCEED model consists of identifying factors that have the potential to influence a given health behaviour. The PRECEDE model classifies them under Predisposing. Enabiing and Feinforcingl factors. According to this model any given behaviour can be explained as a function of 
the collective influence of these three types of factors. Several social psychological theories have been developed that relate Predisposing. Enabling and Reinforcing factors to human behaviour. Attitude-behaviour theories as defined by Fishbein \& Ajzen (1975) and Ajzen \& Madden (1986) and Bandura's Social Cognitive Theory (Bandura, 1986) are examples of more general social psychological models of human behaviour, while the Health Belief Model (HBM) (Rosenstock, 1974) and Protection Motivation Theory (Rogers, 1983) have been specifically developed to account for differences in health-related behaviours.

First of all, social psychological models of human behaviour $(\$ 1.4 .1)$ and social psychological models for health behaviour $(\$ 1.4 .2)$ will be described. After the presentation of these psychological theories, all the theoretical concepts mentioned in these models will be classified under Predisposing. Enabling and Feinforcing factors (5 1.4.3). Subsequently, these Predisposing, Enabling and Reinforcing factors will be adapted to behaviour aimed at STD prevention (\$ 1.4.4) and to behaviour aimed at early STD detection (\$ 1.4.5).

\subsubsection{Soclal psychological models of human behaviours}

\section{Theory of Reasoned Action and the Theory of Planned Behavlour}

The Theory of Reasoned Action (Fishbein \& Ajzen, 1975; Ajzen \& Fishbein, 1980 ) is based on the assumption that human beings make systematic use of the available information. The model assumes that the performance or nonperformance of (recommended) behaviour is primarily a function of the person's intention to perform that behaviour. Important determinants of behavioural intentions are the attitude foward performing the behaviour and the social norm. Peoples' attitudes are their feelings of (un)favourableness towards performing the behaviour and can be measured by assessing the person's beliefs and their ovaluations about benefits of and the barriers to the behaviour of interest. A person's subjective norm is the person's perception that important others think they should perform the behaviour in question and their motivation to comply with those individuals. According to the theory, attitudes and social norms are the sole determinants of intentions. The effects of other variables on intentions are assumed to be mediated by the attitude towards the behaviour, the subjective norm and the relative weights of these components. Additionally, the Theory of Reasoned Action views behaviour change as a matter of changing the cognitive structure underlying that behaviour.

Recently. Ajzen and Madden (1986) suggested adding another variable, perceived behavioural control, as a determinant of intentions to the original model of reasoned action. Perceived behavioural control refers to the perceived ease or difficulty of performing the specific behaviour and is assumed to reflect past experience as well as anticipated barriers. Perceived behavioural controll is 
defined in the same terms as Bandura's (1986) concept of sell-efficacy, a persons' estimation of their abilities to perform the behaviour of interest in a specific situation (Kok, De Vries, Muclde \& Stretcher, 1991; Weinstein, 1993) and must be separated from the abilities or skills a person already possesses (or actual control).

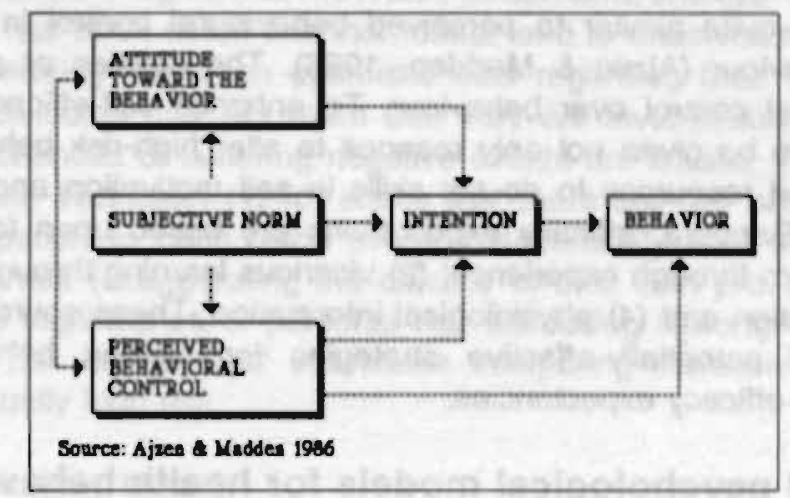

FIGURE 3. THEORY OF PLANNED BEHAVIOUR

At the most basic level of explanation, Ajzen's Theory of Planned Behaviour (see Figure 3) proposes that behaviour is a function of three kinds of beliefs: behavioural beliefs (which are assumed to influence attitudes towards the behaviour), normative beliefs (which constitute the underlying determinants of subjective norms) and control beliefs (which provide the basis for perceptions of behavioural control). The degree of success of predicting actual behaviour will not only depend on one's intention, but also on factors such as availability of requisite opportunities and resources. These variables represent the person's actual control over their behaviour. The dotted arrow in Figure 3 indicates that the association between perceived behavioural control and behaviour is only expected to emerge when there is some agreement between perceptions of control and the person's actual control over the behaviour. If a person has the required abilities and resources, and intends to perform the behaviour, to that extent, the person should succeed.

\section{Soclal Cognitive Theory: self-efflcacy expectations and modeling}

Social Cognitive Theory (Bandura 1986) assumes that protective health behaviours are self-regulatory processes determined by incentives and expectations about (1) consequences of one's own behaviour (opinions about how behaviour is likely to influence outcomes; outcome expectations) and (2) expectations about one's own competence to perform the behaviour needed to influence the outcomes (seff-efficacy expectations). Additionally, expectations about environ- 
mental cues, according to Rosenstock, Stretcher and Becker (1988) similar to perceptions of vulnerability and severity (threat), are also supposed to influence self-regulatory processes. Incentives are defined as the values of particular objects or outcomes, for example, physical appearance or approval of others.

Perceived self-efficacy, the individual's belief that one can exert control over (intentions) behaviour and the social environment forms a central concept of the theory and is quite similar to perceived behavioural control in the Theory of Planned Behaviour (Ajzen \& Madden, 1986). The abilities or skills, however, form the actual control over behaviour. To enhance self-efficacy expectations people need to be given not only reasons to alter high-risk behaviour but also the means and resources to do so: skills in self motivation and self-guidance. According to Bandura, efficacy expectations are based upon four sources (1) individuals learn through experience; (2) vicarious learning through modeling; (3) verbal persuasion and (4) physiological information. These sources may lead to a number of potentially-effective strategies for altering behaviour through modifying self-efficacy expectancies.

\subsubsection{Soclal psychological models for health behaviour}

There has been a gradual development of models to explain or modity health behaviour in the last twenty years. Research on determinants of health behaviour has revealed several unitying principles pertaining to decision-making. Useful models for organizing this knowledge are the Health Belief Model (Rosenstock, 1974; Maiman \& Becker, 1974, Becker \& Joseph, 1984) and the Protection Motivation Theory (Rogers, 1983). Central concepts in these theories are (1) the perception of oneself as being at risk and relating risk to one's actions and (2) the perceived effectiveness of behaviour change and response efficacy.

The Health Bellef Model and the concept of unrealistic blas

According to the Health Belief Model (Maiman \& Becker, 1974; Becker \& Joseph, 1984) people evaluate their risk for particular diseases rationally and change their behaviour accordingly. The model assurnes that people will most likely follow a health recommendation if they have an incentive or a motive to do so and if they hold four separate beliefs, namely (1) that they are vulnerable to the disease or to its consequences; (2) that the disease or its consequences have a serious negative impact on their lives: (3) that following a particular set of recommendations will be effective in reducing the severity of the disease; (4) that psychological and other costs or barriers associated with following the health recommendations are outweighed by their benefits. Psychological barriers include economic costs, convenience factors, and the number and 
severity of side effects. Diverse demographic characteristics, personality factors (e.g. perceived ability to control sexual impulses) and social factors (e.g. social network affiliation and group norms, alcohol and drug use) are included in the model as modifying factors, affecting taking action only indirectly via the other components of the model. The theory adds that this process is triggered by a specific cue to action, a signal that motivates behavioural change.

Nevertheless, it has been noted that individuals tend to underestimate their own vulnerability, operating under an optimistic bias regarding their health Weinstein, 1983). Individuals may not claim that they are invulnerable, but they do claim that their chances of suffering negative events are smaller than the chances of their peers. Weinstein (1990) states that there are two basic processes that could lead poople to claim below average vulnerability: they could underestimate their own risk (exaggerating the efficacy of their own preventive actions and denying the significance of personal risk increasing factors) or they could exaggerate the risk of 'average' individuals comparing themselves to people who are at unusually high risk.

\section{Protection Motivation Theory}

Protection Motivation Theory (PMT) is closely related to the HBM. In a recent revision, Rogers (1983) has added additional cognitive mediating processes (see Figure 4).

In PMT, the sources of information (environmental and intrapersonal) initiate two appraisal processes: threat appraisal and coping appraisal. These cognitive processes involve: a) either maladaptive or adaptive responses and b) the variables increasing or decreasing the probability of the occurrence of these responses. The threat appraisal process evaluates the factors associated with the response that elicits the danger. The variables increasing the probability of the occurrence of the maladaptive response include intrinsic and extrinsic rewards. The factors, decreasing the probability of the maladaptive response are the severity of the danger and one's vulnerability to it. The coping appraisal process evaluates one's ability to cope with and avert the threatening danger. The factors increasing the likelihood of the adaptive response include the efficacy of the response (outcome expectations) and one's ability to perform it successfully (self-efficacy). Figure 4 shows that these two cognitive processes mediate the persuasive effects of fear by eliciting protection motivation, an intervening variable that arouses, sustains and directs actions to cope with danger. 


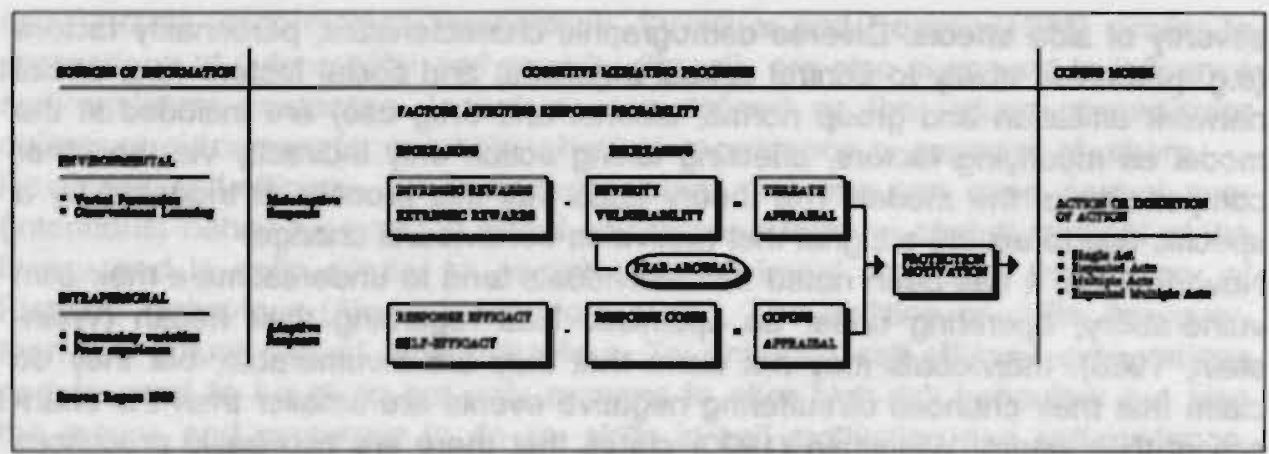

FIQURE 4. PROTECTION MOTIVATION THEOFY

\subsubsection{Predisposing, onabling and reinforcing factors}

Based on cumulative research, three broad groupings of factors are distinguished in the PRECEDE-PROCEED model, that have the potential to influence behaviour, namely: Predisposing, Enabling and Reinforcing factors (see Figure 5). The theories discussed in $\$ 1.4 .1$ and $\$ 1.4 .2$ delivered a lot of different key variables. Yet, in a review of four theories of health-protective behaviour Weinstein, 1993) pointed out the simularities and differences of key variables among these theories (HBM, Protection Motivation Theory, Subjective Expected Utility Theory and the Theory of Reasoned Action). According to Weinstein (1993) several key variables have the same underlying meaning. For example, the expected aversiveness of the outcome is discussed in terms of perceived severity of health consequences in the HBM and Protection Motivation Theory, and as negative evaluation in the Theory of Reasoned Action; likelihood that an outcome will occur is usually referred to as perceived vulnerability in the HBM and Protection Motivation Theory and as expectancy in the Theory of Reasoned Action.

For the purpose of ordering the various variables and notwithstanding these simularities we classified key variables of the separate theories in their original names under Predisposing. Enabling and Reinforcing factors. In Table 3 the educational diagnosis is presented in more general terms and variables are categorised according to predisposing, enabling and reinforcing factors. 
TABLE 3. EDUCATIONAL DIAGNOSIS

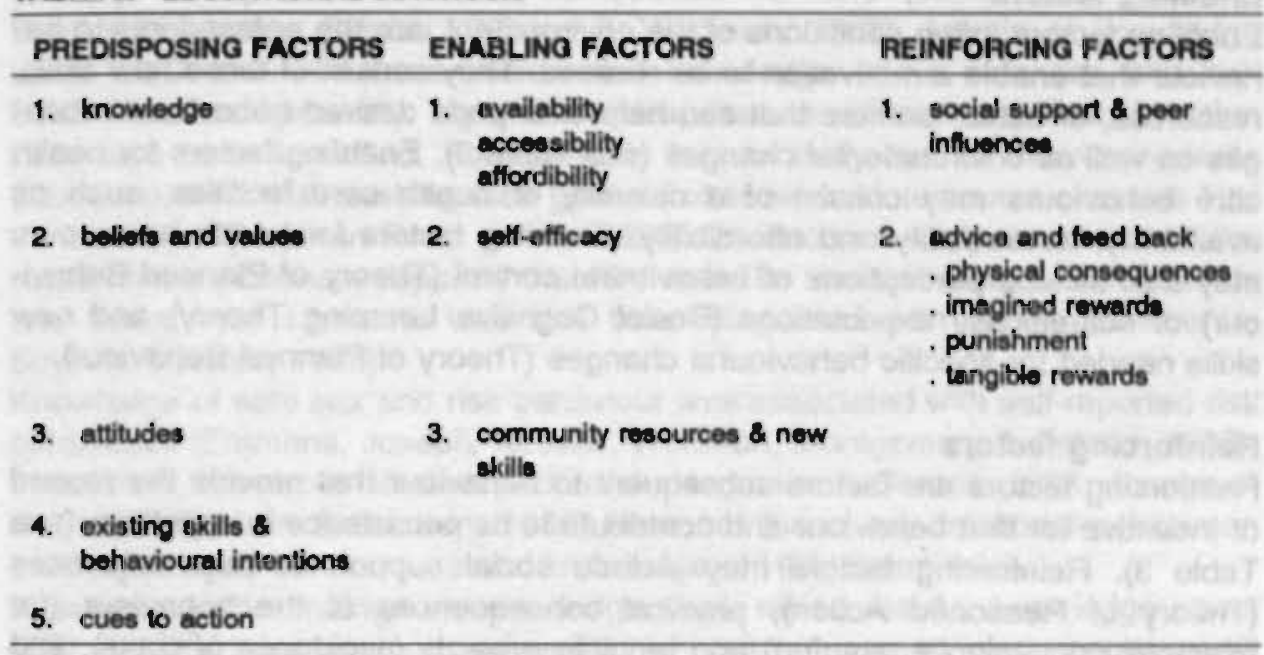

\section{Predlsposing factors}

Predisposing factors are those antecedents to behaviour that provide the rationale or motivation for the behaviour. They may include a number of variables that facilitate or impede motivation to change (see Table 3). A variety of demographic and personality factors could also predispose a given healthrelated behavior. According to the different theories these factors only influence behaviour through their mediating effects on other variables. These factors are not considered here because they cannot be easily and directly influenced by a health promotion programme. Nevertheless, such factors are useful for segmenting populations into subgroups and to determine whether different interventions should be planned for different groups.

Predisposing factors may include a person's knowledge about specific diseases, beliefs of consequences of behaviour and the evaluation of these consequences composing the person's attitude toward the recommended behaviour (Theory of Reasoned Action), or in other words: benefits of and barriers to the behaviour (Health Belief Model), outcome expectations or perceived effectiveness of the behaviour (Social Cognitive Theory), perceptions of vulnerability and severity (Health Belief Model), the concept of unrealistic optimism (Weinstein, 1983) and cues to action (Health Belief Model). Habits or existing skills (Theory of Planned Behaviour) may predispose a person to act in a particular way. The Theory of Reasoned action holds that the final step in the predisposing process is formulating a behavioural intention. 


\section{Enabling factore}

Enabling factors, often conditions of the environment, are the antecedents to behaviour that enable a motivation to be realised. They consist of those new skills, resources, or actual barriers that can help or impede desired behavioural changes as well as environmental changes (see Table 3). Enabling factors for health care behaviours may consist of a diversity of health care facilities, such as availability, accessibility and affordibility. Enabling factors for health behaviours may also include perceptions of behavioural control (Theory of Planned Behaviour) or self-efficacy expectations (Social Cognitive Learning Theory) and new skills needed for specific behavioural changes (Theory of Planned Behaviour).

\section{Relnforclng factors}

Reinforcing factors are factors subsequent to behaviour that provide the reward or incentive for that behaviour and contribute to its persistence or repetition (see Table 3). Reinforcing factors may include social support or peer influences (Theory of Reasoned Action), physical consequences of the behaviour (for example: convenience, comfort) and tangible rewards (avoidance of costs), and imagined or vicarious rewards (improved appearance, self-respect, or association with an admired person). Punishment includes adverse consequences of the behaviour.

\subsubsection{The application of predisposing, enabling and reinfor- cling factors on STD-preventive behaviour}

A review of the literature on predisposing, enabling and reinforcing factors as determinants of condom use will be discussed below. Table 3 is taken as the basis for ordering the research findings.

\section{PREDISPOSING FACTORS AS DETERMINANTS OF STD-PREVENTIVE BEHAVIOUR}

\section{Knowledge}

Knowledge of STD/HIV transinlssion and knowledge of safe sex guldelines Knowledge is generally assumed to be a prerequisite of preventive health behaviour. One must know how STDs and HIV are transmitted to recognise when one is at risk for contracting an STD. Several surveys have been conducted among adolescents to examine their knowledge about STD and HIV transmission and knowledge of safe sex technigues (Simon \& Das, 1984; DiClimente, Zorn \& Temeshok, 1986; Koopman, Rotherman, Henderson, Bradley \& Hunter, 1990; Hingson, Strunin \& Berlin, 1990; Hopman-Rock, Danz, Visser \& Vogels, 1990), arnong STD clinic attenders (Luykx, Marsman \& Van 
der Rijt, 1987) and men with homosexual contacts (Joseph, Montgomery. Emmons, Kessler, Ostrow, O'Brien \& Wortman, 1987). Levels of knowledge about STD and HIV transmission and knowledge about safe sex and risk behaviours are generally high, but widespread misconceptions in information have also been identified. For example, in one study half of the respondents (students) think that washing their genitals after sexual intercourse prevents HIV transmission (Hopman-Pock et al., 1990). In another study one-third of the respondents incorrectly reported that HIV could be transmitted by shaking hands and being near someone who has been diagnosed with AIDS (DiClimente, Boyer \& Morales, 1988).

Knowledge of safe sex and risk behaviour was associated with self-reported risk behaviours (Emmons, Joseph, Kessler, Wortman, Montgomery \& Ostrow, 1986). In contrast, numerous studies (McKusick, Hortsman \& Coates, 1985; Joseph et al., 1987; Baldwin \& Baldwin, 1988) have not found any relationship between the frequency of condom use and knowledge of HIV transmission.

In summary, the findings on knowledge show mixed results: knowledge about STD and HIV transmission is generally high, but widespread misconceptions still persist. In addition, no consistent relationship exists between knowledge of STD/HIV transmission and the frequency of condom use.

\section{Bellefs and values}

\section{Outcome expectations or percelved effectiveness of condom use}

Individuals need to believe that, with regard to contracting STDs, the recommended behaviour will actually make a difference for them. In a study by Emmons et al. (1986) an association was found in a group of homosexual men belween perceived effectiveness of behaviour change and behavioural change, except in groups perceiving themselves to be at highest risk; the authors suggested that those at high risk perceived a behavioural change to be too late for them. Kegeles, Adler and Irwin (1988) noted that although adolescent's perception of condom use as an effective method of preventing STDs is high and the value of condoms attached to avoiding STDs also remained high, these views are not related to increased intention to use condoms. Additionally, a recent study revealed that adolescents who use condoms do not differ from those who do not use them on perceived effectiveness of condoms in preventing STDs (Chapman, Stoker, Ward, Porritt \& Fahey, 1990).

To summarise, these studies suggest that although the effectiveness of condom use is generally supported, these perceptions are not related to actual condom use among adolescents, while they are related to condom use among homosexual men. 


\section{Perceptlons of pereonal risk or vulnerabllity and unreallstic optimlsm}

Perceived vulnerability refers to the likelihood of experiencing personal harm if no preventive action is undertaken. According to the $\mathrm{HBM}$, individuals who believe they are vulnerable to a disease are more likely to engage in behaviours designed to prevent the disease than those who do not.

A study by Simon and Das (1984) on beliefs about STDs and STD-preventive behaviours showed that one-third of college students perceived themselves vulnerable to contracting STDs. Moreover, a study by Cochran and Peplau (1991) showed that one in five psychology students stated there was a chance they could contract herpes and less than one in five claimed a chance of contracting HIV infection. Additionally, based on samples of clinic attenders some researchers (James, Gillies \& Bignell, 1991; Bownes, O'Gorman \& Dinsmore, 1990; Van der Velde \& Hooykaas, 1991) concluded that while the majority of the participants were at increased risk of HIV infection only a small part of the samples perceived themselves at risk of HIV infection.

A review of research findings on determinants of various health behaviours showed that perceptions of vulnerability are related to preventive health behaviours (Janz \& Becker, 1984). A study by James et al. (1991) showed that changes in sexual behaviour, such as increasing condom use, were reported more frequently by individuals who perceived themselves at risk of contracting HIV. Similar findings were also reported in several studies among gay men (McKusick, Coates \& Morin, 1990; Ekstrand \& Coates, 1990) and among college students (DiClimente, Forrest \& Mickler, 1990) but not among clinic attenders in San Francisco (Lindan, Kegeles \& Hearst, 1990) and among New York City students (Reuben, Hein \& Drucker, 1988). Despite these findings, it has been noted that heightened perception of risk does result in behavioural changes, but the effects may diminish over time (Allard, 1989; Becker \& Joseph. 1988; Emmons et al., 1986).

Nevertheless, research findings also indicate that people often fail to recognise their personal vulnerability to disease and instead see themselves as uniquely invulnerable (Weinstein, 1980). If individuals are unrealistically optimistic they will tend to feel less vulnerable and hence are expected to be less likely to intend to use condoms. Numerous groups of researchers reported that some gay men tend to regard themselves as less likely to develop AIDS than other gay men (age-contemporaries or friends) (Bauman \& Siegel, 1987; Joseph et al., 1987; McKusick et al., 1985). Bauman and Siegel (1987) concluded that especially those practicing high-risk sexual behaviours underestimate the riskiness of their sexual behaviour. Van der Velde and Hooykaas (1991) also assessed a tendency to show an optimistic bias for contracting HIV among Amsterdam STD clinic attenders. Contrary to other findings, these researchers found a positive relationship between optimism and intentions to reduce risk; 
their results also revealed that optimists showed lower levels of subsequent behavioural risks. They concluded that optimists were not unrealistically optimistic, but rather that pessimists were unrealistically pessimistic about their vulnerability to contracting HIV (Nan der Velde \& Hooykaas, 1991).

To summarise, a majority of college students and STD clinic attenders do not find themselves vulnerable to acquire an STD or HIV infection. The findings about associations between perceptions of vulnerability and intended condom use are not entirely consistent. In general, perceived vulnerability has been found to be related to reductions in high-risk sexual behaviour and numbers off sex partners, independent of knowledge. The associations between unrealistic optimism and high-risk sexual behaviour also show mixed results.

\section{Perceptions of severity}

Perceived severity denotes the amount of harm that will be experienced if the disease does occur. Simon and Das (1984) found that the majority of a sample of college students perceived STDs as moderately severe. In contrast, another study among Dutch adolescents (Hopman-Fock et al., 1990) showed that almost all adolescents perceive an STD as very severe. Yet, it may be supposed that perceptions of severity of AIDS as a sexually transmitted disease played a role in their statements about the severity of STDs.

In line with the expectations, some researchers (Van der Velde \& Hooykaas, 1991) found that heterosexuals with multiple sex partners attending an STD clinic perceive AIDS as very severe. While they found a significant relationship between severity and intended condom use, the practical meaning of this relationship was somehow negligible 0,2 difference in mean scores on a fivepoint scale). In addition, several studies support the finding that many adolescents consider AIDS as a severe disease (Notermans, 1987; Hopman-Rock et al., 1990; Baldwin \& Baldwin, 1988; Hinghson et al., 1990). In a prospective evaluation of the efficacy of the HBM (Montgomery, Joseph. Becker, Ostrow, Kessler \& Kirscht, 1989) it was found that perceived vulnerability appeared to be of little importance in predicting HIV-preventive behaviours, while perceived severity had the largest (but also of minor importance) beneficial impact on the behaviour.

The above findings suggest that individuals perceive an STD as a moderate disease, while in most cases AIDS is viewed as a very serious disease. Additionally, a rather small relationship holds between perceived severity of AIDS and intended condom use. 


\section{Attitudes}

\section{Attitude towarde condom use}

The decision to use condoms may be determined by an analysis of benefits of and barriers to engaging in condom use behaviour. Barriers to condom use may range from easily remedied problems, for example, not knowing where to get condoms to very complex problems involving extramarital sexual partners.

Previous studies identified broad categories of attitudinal barriers as reasons for not using condoms, such as ignorance and impaired pleasure (Hart, 1976). Recently, Chapman and Hodgson (1988) found a range of negative attitudes towards condom use among heterosexuals with multiple partners. These attitudes include beliefs about condoms as diminishers of sexual pleasure, as interferring with the sexual act and as possibly preventing sex from happening because of the discussion it might bring about by suggesting to use condoms, the belief that condoms are signs of juvenile sexuality and the unerotic nature of condoms. However, these studies fail to demonstrate whether these attitudes predict actual condom use.

In general, the attitude towards condom use is positive among adolescents and is positively related to condom use (De Wit, Kok, Timmermans \& Wijnsma, 1989; Schaalma, Kok \& Peters, 1993). Adolescents and gay men mentioned several barriers of condom use, such as viewing condoms as diminishing sexual pleasure and interrupting sex (Hingson et al., 1990; Turtle, Ford \& Habgood, 1989; MacDonald, Wells, Fisher, Warren, King, Doherty \& Bowie, 1990; Vogels \& Danz, 1990; Ross, 1988), the belief that condom use is a sign of distrust (among gay men) (Ross, 1988), embarrassment about purchasing condoms (college students) (MacDonald et al., 1990), difficulty in discussing condom use with partners (among adolescents) (MacDonald et al., 1990). However, a study among Canadian adolescents (Varnhagen, Svenson, Godin, Johnson \& Solmon, 1991) showed no difference in certain attitudinal barriers (buying condoms in a store, reducing sensation, interfering with sexual spontainety) between those who never and those who always used condoms.

In summary, adolescents claim a positive attitude towards condom use. In samples of adolescents and gay men the elements on which these attitudes are based, can be divided into benefits of and barriers to condom use. Some researchers found a positive association between the attitude towards condom use and actual condom use, while others did not find any association. From the above findings it can also be concluded that no specifically described behaviour (such as condom use with a casual partner the next time when having sexual intercourse) has been investigated in most studies. 


\section{ExIsting skllis and behavioural Intentions}

\section{Previous condom use and intentlon to use condoms}

The majority of the adolescent population presently never use or irregularly use condoms Nogels \& Van der Vliet, 1990; Gotlieb, Vacalis, Palmer, \& Colon, 1988; Strunin \& Hingson, 1987; Kegeles et al., 1988; Schaalma et al., 1993). Previous condom use was related to the intention to use condoms (BasenEngquist, 1992; Van der Velde \& Van der Pligt, 1991) and may reflect behavioural stability over time. Yet, in a random sample-telephone survey of the general population on the effects of the AIDS campaigns, a major discrepancy between intented condom use and previous condom use was found (De Vroome, Paalman, Sandfort, Sleutjes, de Vries \& Tielman, 1990).

In summary, most results indicate a relationship between previous condom use and intended condom use by adolescents.

\section{Cues to action}

Cues to actlon: medla messages

The HBM model postulates that (besides the components of vulnerability and severity and an analysis of benefits and costs) when a cue to action (e.g. mass media message) is present, the appropriate health behaviour may be elicited. Before the AIDS epidemic, there had been one national mass media campaign in the Netherlands about STDs aimed at the general public, the SIRE campaign (in 1985). The major objectives of this campaign were to increase the level of knowledge of STDs, to stimulate a more positive attitude towards the prevention of STDs and to promote behavioural changes. A study on the evaluation of the effectiveness of the SIRE campaign revealed that rather small and only short term effects existed on knowledge between the exposed and non-exposed groups (such as knowledge about prevention of STDs, knowledge about different types of STDs, condom use as means of STD prevention, higher intention to warn sexual partners) (Marsman, Van der Rijt \& Luykx, 1987).

The approach of AIDS prevention in the Netherlands has been characterised by an emphasis on facts (De Vroome et al., 1990). Until 1987 campaigns aimed at preventing HIV transmission were mainly focused on men with homosexual contacts, intravenous drug users and hemophiliacs. Since 1987, several national campaigns have been developed to control HIV infection (and other STDs), for example, the national condom promotion campaign aimed at the general public. These "behaviour campaigns" were mainly targeted at attitude and behavioural changes and promoting safe-sex techniques, such as condom use, postponing sexual intercourse, restricting sexual contact to a mutually monogamous relationship and practicing nonpenetrative sexual techniques. Moreover, the 
Dutch Ministry of Welfare, Public health and Culture developed a national campaign aimed at spreading the knowledge about HIV infection and AIDS. To inform the general population, mass media was used, such as: iv and radio commercials, printed advertisement, posters and flyers. Since then, several studies have taken place to gain a better understanding of the effects of these campaigns. Firstly, by conducting successive population surveys, De Vroome et al. (1990) assessed the effects of several years of campaigning. It appears that knowledge about the prevention of HIV transmission by using condoms has reached $98 \%$ of the sample. During the study there was an increase in the number of individuals who expressed an intention to use condoms $(23 \%$ in 1987 to $33 \%$ in 1989) or who were already using them. According to De Vroome et al. (1990) the observations are confirmed by condom sales figures and STD incidences. In addition, a second study among a representative sample of the Dutch population showed that Dutch individuals are well informed about AIDS, transmission routes, and condom use as a preventive means of HIV transmission, but a large part of the population still does not use condoms on a regular basis Nan Zessen \& Sandfort, 1991). Sherr, Strong and Goldmeire (1990) also concluded that health education campaigns had no apparent effect on determining whether STD clinic attenders used condoms or not; this was in spite of the high endorsement of the messages and the influence they had on the subjects' recorded feelings of influence.

Another indirect reflection of possible behavioural changes are the trends in visits of two STD clinics in Amsterdam. These trends showed that the number of male and female heterosexual visitors declined from 1987 to 1989. At the same time the number of customers reported by prostitutes in the sample declined noticeably and condom use with casual sexual partners tended to increase in the sample pericd. Since 1989, however, the number of STD clinic attenders has started to increase again (Van Haastrech et al., 1991). In addition, a significant decline in the number of notified gonorrhea and syphilis cases; among the general public $(G H I)$ has been observed since 1981, thus preceding the onset of the AIDS epidemic and the mass media campaigins. Yet, a remarkable decline of the incidence of rectal gonorrhea among homosexual and bisexual men has been observed in Amsterdam since 1983 (Van de Laar et al., 1990). This coincided with a focus on HIV transmission targeted at homosexual rnen in Amsterdam.

Another objective measure of possible behavioural changes; comes from the overall sale of condoms, which has increased by an average of $30 \%$ since 1987 . which is when the national campaigns aimed at the general public were started (SOA Stichting, 1988).

To summarise, some objective measures indicate that behavioural changes have developed since 1981. The developments found might be attributed to the 
campaigns, but other factors may also have influenced these behavioural changes.

\section{ENABLING FACTORS AS DETERMINANTS OF STD-PAEVENTIVE BEHAVIOUA}

\section{Avalilbility, acceeslbility and affordibility}

\section{Actual barrlers: access to and costs of condoms}

It has been assumed that the price of condoms and the location where they are sold, are important barriers to using condoms. Four kinds of distribution channels for condoms have been identified: health service provision, communitybased distribution, social marketing programmes and established commercial channels (Spencer, 1990). In the United States many supermarket chains started selling condoms in 1987. In 1988 food stores accounted for $15 \%$ of condom sales in the U.S., while drug stores accounted for $64 \%$, discount stores and vending machines $10 \%$ each, and mail order $1 \%$ (Murphy, 1990). In the Netherlands condoms can be purchased at drug stores, vending machines, in discos, and supermarkets. Drug stores accounted for $60 \%$ of the market in the Netherlands. According to the London Rubber Company condom sales have increased steadily since 1986 . No research has been carried out on perceptions of costs and availability of condoms on the one hand and actual condom use on the other.

\section{Community resources and new skills}

\section{Skills in the management of sexual activities}

Human competence requires not only self-efficacy expectations, but also actual skills in the management of self-protective control over sexual situations. Gilchrist and Schinke (1983) taught adolescents, through modeling, how to communicate and negotiate about sexual matters and contraceptives. They let the adolescents practice these social skills by role playing and giving constructive feedback. This programme enhanced perceived self-efficacy and skills in managing sexual activities. In addition, a study among gay men on exercising self-protective behaviour in sexual relationships found that they became more skilful in dealing with sexual relationships and resisting to coercion, reduced high-risk sexual practices and used condoms on a regular basis than the matched control subjects (Kelly, St. Lawrence, Hood \& Brasfield, 1989).

These findings suggest that training in skills enhances self-efficacy expectations and that possessing actual skills in the management of sexual activities are related to condom use. 


\section{Solf-officacy oxpectations}

\section{Self-efficacy or percelved behavioural control}

Individuals need to be able to carry out behaviours that ultimately lead to using condoms in different sexual situations. Self-efficacy expectations, the individual's belief that one can exert control over behaviour, is significantly related to condom use among high-risk adolescent females (Kegeles, Adler \& Irwin, 1989). In a study among Dutch adolescents (Richard et al., 1991) it was concluded that self-efficacy expectations had a strong effect on previous condom use by females, but this factor explained only little variance of the males' condom use. A similar study (De Wit ef al., 1990) showed that apart from the attitude toward condom use and the social norm, self-efficacy explained some variance in intention to use a condom with an unknown partner, while no relationship existed between self-efficacy expectations and intended condom use with a known partner. In addition, Basen-Engquist (1992) found that self-efficacy was related directly to the intention to discussion behaviour (about STDs and AIDS) and indirectly (via perceived barriers to preventive behaviour) to the intention to use condoms.

Once an individual has decided to use condoms, negotiating their use with their sexual partner may require some basic sexual communication skills. Past research on communicating skills of adolescents indicated that those teenagers who are better communicators are more effective contraceptive users (Cvetkovich \& Grote, 1981; Polit-O'Hara \& Kahn, 1985). On the contrary, a study on determinants of condom use as an STD/HIV preventive measure showed that it are not the general communication skills, but only specific communication skills with regard to condom use that were related to condom use (Catania, Dolcini, Coates, Kegeles, Greenblatt \& Puckett, 1989). These discrepancies may be related to differences in the focus on disease versus pregnancy prevention.

To summarise, the influential role played by self-efficacy expectations in the management of sexua! activities, specifically condom use, is documented in the above studies on contraceptive use as well as on STD preventive use.

\section{REINFORCING FACTORS AS DETERMINANTS OF STD-PFEVENTIVE BEHAVIOUR}

\section{Soclal support and peer Influence}

\section{Social influence}

Perceived social norms, the individual's beliefs what their pesers think of the recommended behaviour, have repeatedly been reported to be a significant predictor of the use of contraceptives (Adler, Kegeles, Irwin \& Wibblesman, 1990). Perceived popularity of condoms also revealed to be related to condom use among males and females (Kegeles et al., 1989). The salience of social 
factors in condom use may be obvious when considered that every sexual risk situation is a social transaction. A study among homosexual men showed that peer influences (beliefs of friends and pantners) were associated with behavioural changes (McCusker et al., 1989a). Another study found that women who felt that condoms could facilitate sexual enjoyment, that male partners and peers who advocated condom use and felt that condoms should be advertised, were more likely to use condoms (Naldiserri, Arena, Proctor \& Bonatti, 1989). Furthermore, in a study among Dutch adolescents (Schaalma et al., 1993) social norms predicted intended condom use (after attitude and self-efficacy expectations). Yet, in a study by Richard et al. (1991) social norms were not found to be a predictor of previous condom use.

In summary, mixed results were found regarding the influence of social norms on intended condom use.

\section{Advice and feed back}

\section{Relnforcement and punishment}

Reinforcement is the reward of desirable (condom use) as well as of undesirable behaviour (not using condoms). For example, a reinforcing factor might be the money which prostitutes earn by having unprotected sexual intercourse with a customer or the idea of being optimally protected by having protected sexual intercourse. Punishment includes the negative consequences of desirable and undesirable behaviour. A negative consequence of unprotected sexual intercourse might be the contracting of STDs. Yet, the chance of contracting an STD by having one unprotected sexual contact is relatively low. In behavioural terms this indicates that in most cases having unprotected sexual intercourse yields no short term negative consequences. Unfortunately, no data are available on the influence of reinforcing and punishing factors on STD-protective actions.

\subsubsection{The application of predisposing, enabling and reinfor- cing factors on early STD detection}

Apart from a vaccination for hepatitis B, no vaccines are available for other STDs including HIV infection. Successful treatment of STDs depends on early detection of these diseases (Cates \& Meheus, 1990). As mentioned before, early identification of Sexually Transmitted Diseases includes actions, such as self-examination and self-diagnosis, regular asymplomatic check-ups on STDs and prompt help-seeking behaviour in case of complaints or symptoms, or after partner notification. Until now little research has been carried out on determinants of STD self-examination, periodic clinical examination and prompt helpseeking behaviour, with some notable exceptions which will be discussed here. 
Thus, the absence in the presentation of research findings below indicates that, according to us, no research has been carried out on relationships between PRECEDE-factors and early detection behaviours. On the contrary, several other self-examination behaviours, such as Breast Self-Exarnination (BSE) (Shepperd, Solomon, Atkins, Foster, Frankowski, 1990; Champion, 1990), skin examination behaviour (Cody \& Lee, 1990) and testicular self-examination (Brubaker \& Wickersman, 1990) have been explored in detail. Owing to the absence of studies on STD self-examination behaviour the results of determinants of other self-examination behaviours will be presented here. Because of the stigmatised aspects surrounding STDs and the absence of acute life-threatening situations in STDs (not HIV infection) it does not seem relevant to compare the delay in responding to signs and symptoms of STDs to delay behaviour in patients with symptoms of other diseases, such as myocardial infection or cancer. Additionally, attitudes, perceptions of vulnerability and severity have already been discussed in more general terms in the paragraph on "determinants of condom use". To prevent repetition, this part focuses only on the relationship between the separate concepts (e.g. severity, vulnerability, attitudes) and (STD) self-examination, periodic clinical examinations and prompt help-seeking behaviour, respectively.

\section{PREDISPOSINO FACTORS AS DETERMIMANTS OF EARLY STD DETECTION}

\section{Knowledge}

Knowledge about STDs and STD-related symptoms

Individuals at risk of contracting STDS should know the major signs and symptorns of common STDs and should understand that asymptomatic infections are common. A recent study among 11,431 Dutch adolescents (HopmanRock ot al., 1990) shewed that half had never heard of gonorrhea, about twothirds had never heard of herpes and hepatitis B, and three-quarters had never heard of syphilis. Additionally, almost one-third did not kncis that STDs can be asymplomatic, and $37 \%$ did not know that STDs do not disappear spontaneously. Knowing where to seek medical treatment if one suspects an STD is likely to be an important factor in the case of prompt help-seeking behaviour. Yet, more than half of the adolescents reported not knowing where to go if they suspect an STD (Hopman-Fock et al., 1990).

From these findings it can be concluded that knowledge about STDs, STDrelated symptoms and STD health care facilities is poor among Dutch adolescents. 


\section{Beliefs and values}

\section{Percelved effectiveness of early STD detection behavlours}

The study on Testicular Self-Examination (Brubacker \& Wickersman, 1990) revealed that intenders believed more strongly than non-intenders that performing TSE would result in desirable outcomes (delect cancer in its early state). In addition, studies on other self-examination behaviours also showed that perceived effectiveness of specific BSE behaviours was related to intended BSE (Champion, 1990).

Despite the fact that no research findings are available about the perceived effectiveness of early STD detection behaviours, the above findings suggest that the perceived effectiveness of STD self-examination might influence intentions to early STD detection activities.

\section{Percelved vulnerability and percelved severity of STDs}

One study among college students examined the relationship between vulnerability and severity on the one hand and asymptomatic STD check-ups on the other (Simon \& Das, 1984). They found that perceived severity was not related to the frequency of asymptomatic check-ups. In addition, these researchers showed that students who reported they had gone for asymptomatic check-ups were more likely to perceive themselves as highly vulnerable to contracting an STD. Yet, the predictive power of vulnerability on the frequency of asymptomatic check-ups was rather small. To our knowledge no other research has been carried out to investigate relationships between perceptions of vulnerability and severity on the one hand and STD self-examination, periodic clinical examination and prompt help-seeking behaviour on the other.

Nevertheless, research on BSE and TSE behaviours, similar in many aspects to STD self-examination, showed that perceived vulnerability was found to be associated with BSE (Calnan \& Rutter, 1986; Massey, 1986). Other researchers found a relationship between vulnerability and the frequency of BSE, but not between severity and BSE (Champion, 1985; Rutledge, 1987). No relationships were found between vulnerability and severity on the one hand and prompt help-seeking behaviour in patients visiting their general physician for general complaints on the other (Cassee, 1973). Although, researchers into cancer delay have commonly asserted that great anxiety or fear in response to the discovery of cancer symptoms causes the symptoms to be defensively denied or avoided (Eardley, 1974) a study of Timko (1987) showed that a more extreme reaction of negative feelings was positively related to intentions to seek medical care promptly.

In summary, the above findings suggest that the influence of vulnerability on these various self-examination behaviours is rather small or completely absent. 
In addition, perceived severity was not related to asymptomatic check-ups for STDs and other self-examination behaviours either.

\section{Attitudes}

\section{Attitudes towards early STD detection behaviours}

The attitude towards early STD detection behaviours may influence actual STD self-examination, periodic clinical examination and prompt help seeking behaviour. The study by Simon and Das (1984) among college students revealed that benefits were perceived to be twice as high by those with frequent asymptomatic check ups for venereal diseases than by those who never had such checkups, while barriers were less for those with a history of asymptomatic checkups. In addition, for barriers, approximately one-quarter of the sample appeared to be concerned about pain during STD check-ups or ernbarrassment at the prospect of going for STD check-ups. Approximately one-third of the sample also appeared to be reluctant to disclose their sexual contacts (Simon \& Das, 1984). No other studies have been carried out on the attitude towards early STD detection behaviours.

With regard to various self-examination behaviours, benefits of Breast SelfExamination (BSE) have been related to BSE but the greatest amount of variance in BSE has been explained by perceived barriers, such as: fear about cancer, embarrassment, lack of time, unpleasantness, and difficulty in remembering (Champion, 1985; Lashley, 1987). Also, the attitude toward Testicular SelfExamination (TSE) contributed significantly to intended TSE (Brubaker \& Wickersman, 1990).

In summary, the findings reported here suggest that attitudes toward selfexamination behaviour play a role in some early detection activities. Barriers more than benefits accounted for some of the variance in self-examination behaviours.

\section{Existing skills and behavioural intentions}

Prevlous behaviour and Intentions to early STD detection behaviours When individuals suspect an STD it is necessary to seek medical assessment immediately. About two-thirds of 11,431 Dutch adolescents (Hopman-Rock et al., 1990) stated they would seek medical assessment if they suspect an STD, $15 \%$ would discuss their suspicion with others and $17 \%$ would wait and see. No research has been carried out on the frequency of previous STD self-examination behaviour and of periodic clinical exarninations either. 


\section{Cues to action}

\section{Medla messages}

According to the Health Belief Model a cue to action, internal or external, may influence health behaviour. It has also been assumed that cues to action, such as mass media messages influence anxiety and because of this fear increase the consulting behaviour of persons who are not at risk of contracting an STD. Yet, a study into the effects of mass media campaigns on STD-related consultations showed that among $16 \%$ of a sample of STD clinic attenders $(\mathrm{N}=839)$ mass media campaigns played a role in their decision to seek medical assessment of a suspected STD (Luykx, Marsman \& Van der Rijt, 1988). In addition, persons who visited a physician because of a mass media campaign were slightly more likely to have a negative diagnosis than those who did not receive any information.

\section{ENABLING FACTORS AS DETERMINANTS OF EARLY STD DETECTION}

\section{Avallablilty, accessiblilty and affordibllity}

\section{Avallability, accessibility and cost of treatment}

The existence of specific STD health services may influence periodic clinical examinations and prompt help-seeking behaviour. The formal state of affairs in the Netherlands is that persons who suspect an STD consult a general practitioner (GP) for medical care. Diagnosis and treatment is carried out by the GP, or the patient can be referred to an independent specialist or specialist connected to a hospital. The health insurance company covers the costs of the visit, diagnostic examinations and prescriptions.

Since 1976, the Ministry of Welfare. Public Health and Culture (WVC) has subsidised out-patient STD clinics in the four largest cities (Amsterdam, Rotterdam, The Hague and Utrecht) which have high-quality facilities for examination, diagnosis, treatment and contact tracing, and offer free access and anonymous medical care to all residents of the Netherlands who (1) are not insured; (2) do not have a GP; or (3) wish to remain anonymous because of the taboo on STDs. The patient does not need a letter of referral from a GP in order to obtain treatment in these clinics. Some clinics have an appoimment system, while others are walk-in type clinics. In order to make these "low-threshold facilities: possible in the other towns, the "VoMiL regulation" was set up (named after the then department of public health and environmental affairs). Persons who do not wish to visit a GP can, by virtue of this regulation, be referred to an independent dermatologist or a dermatologist in a general hospital by a social nurse working at a Municipal Health Service (MHS). In addition to these low-threshold out- 
patient STD clinics and institutions with VoMil-regulations, nine MHSs have been allowed to offer (under certain conditions) free and anonymous diagnosis and treatment for venereal diseases since 1985. Apart from the above mentioned health providers and institutions, there are a number of Family Planning Clinics in the Netherlands that deal with STD prevention and cureation. In these institutions, however, the patients have to pay for their diagnosis and treatment themselves.

A recent study on the consumer's view of genitourinary medicine services in the United Kingdom (Munday, 1990) revealed that a majority of patients wanted the out-patient STD clinic to be open in the evenings and to be sited away from the main out-patient departments. Three-quarters of the patients wanted to maintain an open access system although the majority would also like appointment systems to be available. Most patients did not want the results of the consultation to be sent to their GPs without their consent. Until now, no research has been carried out to assess relationships between specific service attributes of STD health care institutions on the one hand and prompt help-seeking behaviour and periodic clinical examaninations on the other.

\section{Communlty resources and new skills}

\section{Now skIIls}

To our knowledge no resarch has been conducted on new skills as determinants of early STD detection behaviors.

\section{Self-efflcacy expectations}

\section{Seli-offlicacy}

Recent studies have supported the proposed link between health-related intentions and self-efficacy expectations (Ajzen \& Madden, 1986). In the study on TSE (Brubaker \& Wickersman, 1990) it was found that self-efficacy expectations improved the prediction of intention to TSE significantly.

From these findings we may assume that self-efficacy expectations might influence early STD detection behaviours as well.

\section{REINFORCINQ FACTOAS AS DETERMINANTS OF EARLY STD DETECTHON}

\section{Soclal support and peer Influence}

\section{Social influence}

The individual's belief of what important others think of their behaviour may influence early detection behaviours. Yet, no studies have been conducted to investigate the influence of social norms on early STD detection behaviours. 
However, various studies have addressed the impact of social influence on Breast Self-Examination (Calnan \& Moss, 1984), while no relationship existed between social norms and BSE in a study by Champion (1990). Intentions of delay in seeking medical assessment for breast cancer symptoms were related to perceived social pressure to delay (Timko, 1987). Additionally, Cassee (1973) found that the social environment influenced the decision to seek medical treatment from a GP for diverse complaints.

From these findings we may conclude that the influence of social norms on early detection behaviours has been established in different studies and from this finding it may be assumed that social norms influence early STD detection behaviours as well.

\section{Advice and feed back}

\section{Relnforcement and punlshment}

No studies have been conducted to examine the influence of feed back on early STD detetcion behaviours.

\subsection{Research questions and design}

The aim of the preceding paragraphs was not only to gain a better insight into the occurrence of STDs and STD-preventive behaviours but also to better understand the determinants of STD-related health behaviours. It has already been noted that central concepts from social psychological models are proved to be determinants of intentions to both using condoms and performing other safe sex behaviours. However, the majority of these measures are operationalised poorly.

The literature reviewed until 1987, the year in which we started our studies among persons seeking treatment for an STD and among adolescents, showed a lack of theoretically based surveys on determinants of behaviours aimed at primary prevention of STDs. Again, until now, few theoretically based studies have been conducted to examine determinants of behaviours aimed at early STD detection. Considering these lacunae in the research literature, we therefore started a study on determinants of STD-preventive behaviours among Dutch adolescents and a study on determinants of early STD detection behaviours among persons seeking medical treatment for a suspected STD. In order to gain more theoretically based insight into the determinants of condom use among heterosexuals, we started a third study among Dutch heterosexuals: requesting an HIV test at MHSs or STD clinics in 1991. The results of these studies are presented in parts II and III. 
In this paragraph the main focus will be on some methodological aspects of these studies. First of all attention will be paid on central research questions to be answered by these studies. Next, the theoretical perspective and methods used to answer the central questions are discussed in more general terms. For a detailed description of specific methods (measures, data analysis and a description of respondents) we refer the reader to the separate research articles about these matters in parts II and III.

\subsubsection{Research questions}

The main purpose of this thesis is to analyse both determinants of STD-preventive behaviour and determinants of early STD detection behaviours, such as STD self-examination, periodic clinical examination and prompt help-seeking behaviour. These studies should not only result in assignments for the content of STD prevention programmes, but also in recommendations for early disease detection activities.

Central questions to be answered are:

1. What factors are related to the intention to perform STD-preventive behaviours, especially condom use?;

2. What factors are related to intentions to perform behaviours aimed at early detection of Sexually Transmitted Diseases?;

3. What is the role of specific service attributes (characteristics of health service institutions) in the choice of STD health care facilities?;

Finally, to link the results of these studies and the literature study to recommendations for policy makers, a fourth question should be answered:

4. What are the implications of the results for the development of interventions and other STD control activities?

\subsubsection{Methods}

\section{The studies}

In order to answer the above research questions three studies were conducted:

- A study on determinants of both STD-preventive behaviours and choice of STD health services among secondary school pupils (adolescents and young adults):

- A study on determinants of STD-preventive behaviours among persons requesting an HIV test (heterosexuals with an HIV testrequest);

- A study on both determinants of early detection of STDs and choice of STD health services among persons seeking a medical examination for a suspected STD (persons with an STD request). 


\section{The study among adolescents and young adults}

In order to answer a part of the research questions 1 and 3, a survey was distributed among adolescents in The Hague, in mid-February 1988. Two schools for intermediate vocational education, three schools for part-time education and the highest grades of four community schools participated in this study. To achieve an even distribution of the various school years, several classes were visited at schools. instructed by the researchers, the questionnaires were completed in class anonymously and then collected in a sealed envelope. A total of 308 pupils were approached to participate in this study. Two pupils refused to cooperate after examining the content of the questionnaires and after opening the envelopes, another 36 respondents were excluded because more than 7 answers were missing. Finally, the questionnaire was more or less completely filled in by 270 pupils.

\section{The study among heterosexuals with an HIV testrequest}

Subjects who attended Municipal Health Services or STD clinics for an HIV test in thirteen regions (major cities in the Netherlands) entered the study in 1991. Individuals who were not proficient in Dutch, respondents who sought for an HIV test after incest and those who came in a second time because of the window period, were excluded from the study. The health care provider encouraged the individuals to participate in the study and then provided them with instructions about answering a questionnaire. All subjects completed the first part of a structured questionnaire after the pretest consultation and the second part after the posttest consultation. Anonymity was guaranteed. Of the persons with an HIV testrequest $20 \%$ refused to participate in the study. In addition, almost $20 \%$ of the 326 respondents did not fill in the second part after the posttest consultation. No differences were found in age, gender and education between respondents who filled in the first and second parts of the questionnaire and those who only completed the first part. Reasons for not filiing in the second part of the questionnaire were: not enough time, first part was too long and some of the questions were too personal, did not return for the testresults, got the testresult by telephone and the health care provider forgot to hand out the questionnaire.

\section{The study among persons with an STD request}

Persons attending health services in eight regions (major cities in the Netherlands) for a suspected (new) STD in 1987 were selected as the study populati. on. Within each region participants were selected from (1) an STD clinic; (2) a Family Planning Clinic; (3) a general practitioner. Individuals who were not proficient in Dutch and those who were attending for a check-up of an old, existing STD were excluded from the study. Health care providers encouraged their patients to participate in the investigation and then provided instructions 
about answering the questionnaire. All subjects completed a structured questionnaire after the individual consultation. Anonymity was guaranteed. At the moment of filling in the questionnaire the respondents were not informed about the results of the examination; their diagnosis.

According to the information gathered from the health care providers an overall response-rate of $92 \%(\mathrm{~N}=855)$ was obtained. Reasons for not filling in the questionnaire were: not enough time, the questionnaire was too long and some of the questions were too personal.

\section{Theoretical perepective and Measuremente}

Concepts from both the Health Belief Model and the Theory of Reasoned Action underly our studies and guided our selection of variables. As mentioned earlier, several key variables of the Health Belief Model and the Theory of Reasoned Action have the same underlying meaning (Weinstein, 1993). Yet, the Theory of Reasoned Action differs from the Health Belief Model by considering a much wider range of consequences of continuing the current behaviour (such as: costs, health outcomes and other nonhealth outcomes), including the consequences from obeying the preferences of important others. When the issue is the adoption of health protective behaviour, health consequences are likely to be among the various consequences salient to people. In the Theory of Reasoned Action these health consequences are not seperated from other consequences. In addition, the Health belief Model assumes that general questions about vulnerability, severity, benefits of and barriers to action might be sufficient to predict protective health behaviour. Because of the latter, we used the Health Belief Model in the studies on determinants of specific health protective behaviors, such as: STD self-examination, periodic clinical examination and patients' delay. In addition, because of the wider range of consequences taken into consideration in the Theory of Reasoned Action we used this theory in the studies on condom use, STD-preventive behaviour and choice of circuits (health care services).

All the studies were conducted by means of structured questionnaires. For a detailed description of the questionnaires and the operationalisation of the different variables used in these studies the reader is referred to the separate articles of parts II and III.

\section{Data-analysis:}

All statistical analyses have been conducted with the software package SPSSPC+ 3.1. (Norusis, 1988). For the description of specific analysis carried out in the different studies we refer the reader to parts II and III. 


\section{GENERAL DISCUSSION}

In Chapter 1 of this overview four central research questions have been presented. These questions will be answered here successively. In $\$ 2.1$ attention will be paid to major findings of the research presented in part II and part III. For a detailed description of the results we refer the reader to part II with regard to the presentation of determinants of STD-preventive behaviours and to part III with regard to factors related to the early detection of STDs. When comparing our results with that of other researchers we mainly pay attention to Dutch studies. This decision has been made because several international studies are already referred to in the articles and in Chapter 1 . Another point to remember is that the results in $\$ 2.1 .1$ are ordered according to Table 3 of Chapter 1. Because the results of the studies on determinants of early detection behaviours concern one study population and because there are not so much variables analysed in these studies, we only present a summary of major findings per behaviour under investigation (independent variable) in \$2.1.2.

In the second part of this chapter some methodological issues will be discussed. Attention will be paid to the generalisability of the results, the problem of causality and self-report measures, the operationalisation of specific variables and the complexity of the behaviours investigated.

In $\$ 2.3$ the implications of the results on STD-related interventions are described with respect to STD prevention, early detection and the importance of special service attributes in STD health care. Recommendations focus on (1) target groups, in which the content of the advocated interventions are discussed; (2) the promotion of early detection behaviour and (3) the varied needs and demands in the organisation of STD health care. Finally, theoretical conclusions and some suggestions for future research are formulated.

\subsection{A summary of major findings}

\subsubsection{Determinants of STD-preventive behaviour}

In order to answer the first central question, namely "What factors are related to the intention to perform STD-preventive behaviour, especially condom use?", the results of three studies are presented. Central focus is on the main concepts of the theories mentioned in $\$ 1.4$ and these concepts again are classified under Predisposing, Enabling and Reinforcing factors in this paragraph (see Table 3). For each concept the research findings are summarised from our studies on (1) determinants of intention to use condoms among sexually active adolescents (see Chapter 4; Leenaars, Rombouts, Lammerdin \& Zwetsloot, 1990); (2) determinants of STD-preventive behaviour of sexually active and sexually inactive adolescents (see Chapter 5 ; Leenaars, Rombouts, Lammerdin 
\& Zwetsloot, 1990) and (3) determinants of condom use among heterosexuals with an HIV testrequest (see Chapter 3; Leenaars, Sandfort, Rombouts, Kok \& Schrijvers, submitted for publication). The reader should keep in mind that not all concepts have been investigated in these different studies. Thus, when describing the results on specific subjects attention will only be paid to the studies which examined these matters. In addition, the reader should also keep in mind that we discuss intentions to behaviour (intended condom use and intended STD-preventive behaviour) and self-reported previous behaviours (previous condom use) in stead of actual behaviour.

\section{PAEDISPOSINO FACTORS AS DETERIMINANTS OF STD-PREVENTIVE BEHAVIOUR}

\section{Knowledge}

\section{Knowledge of STD and HIV transmission routes}

The results of the study among adolescents show that on average these adolescents do know the answers of fourteen out of 22 questions about STDs, they do not know the answers of an average of five out of 22 questions, while they also incorrectly answered an average of three out of 22 questions. The adolescents also find their own level of STD related knowledge poor to reasonably sufficient and of the total population one-third want more information about STDs. In agreement with numerous other studies (Baldwin \& Baldwin, 1988; McKusick et al., 1985) knowledge of different aspects of STDs is not related to the intention to perform STD-preventive behaviour among these adolescents.

To summarise, the findings on knowledge show mixed results: knowledge about STDs is generally high, but widespread misconceptions still exist. In addition, no relationship exists between knowledge and intended STD-preventive behaviour.

\section{Bellefs and values}

\section{Percelved effectlveness of condom use}

The study among adolescents shows that adolescents who always use condoms view condoms as more effective in the prevention of venereal diseases than those who irregularly or never use condoms. Consistent with other findings (Kegeles et al., 1988; Chapman et al., 1990) expected effectiveness of condom use predicts the males' intention to use condoms. Yet, the females' intention is not explained by the expected effectiveness of condom use. One explanation for the observed difference may be that females usually do not feel themselves responsible for the use of condoms.

From these results it appears that expected effectiveness of condorn use only plays a role in the males' intention to use condoms, but not in the females'. 
Perceptions of pereonal risk/vulnerablity and unrealistic optimlem

The results of the study among adolescents show that adolescents estimate their own risk of contracting an STD infection to be very low, while this risk, viewed objectively, is definitely present. This finding has also been assessed by James ef al. (1991) and Bownes et al. (1990). Remarkably, this estimated infection risk shows no relationship with previous condom use.

From the study among persons with an HIV testrequest it can be concluded that a relatively large number of these heterosexuals felt themselves less vulnerable to HIV infection than peers and friends. This may indicate that they show an optimistic infection risk with regard to contracting HIV. These findings are comparable with other studies among gay men and STD clinic attenders (Bauman \& Siegel, 1987; Joseph of al., 1987; McKusick of al., 1985). Yet, this optimistic infection risk does not influence the intention to use condoms with a casual partner. Nearly all heterosexuals estimate their chance of getting HIV infected in the near future to be (very) small. This estimated infection risk does not play a part in explaining intended condom use with a casual partner either.

These findings suggest that adolescents and heterosexuals with an HIV testrequest somehow believe they will not contract an STD or HIV infection. In addition, we may conclude that determinants such as perceived vulnerability, reporting an optimistic infection risk, and the estimated infection risk do not contribute to the prediction of intended condom use.

\section{Perceptions of severlty}

The study among adolescents shows that consistent with Simon and Das (1984) but not with Hopman-Rock et al. (1990) the majority of adolescents do not find an STD a serious disease. In turn, no association has been found between perceived severity and intended condom use with a new partner.

Half of the heterosexuals with an HIV testrequest perceive AIDS as very threatening for the Dutch population. In addition, perceived threat of AIDS reveals to be a moderate predictor in the intention of these heterosexuals to use condoms.

In summary, the results show that the perceived severity of AIDS may lead to preventive behaviour, while the severity of STDs play no part in the intention to use condoms use among adolescents.

\section{Attludes}

\section{Attitude towards STD-preventlve behavlour}

In line with the findings of others (Hingson et al., 1990; Schaalma ef al., 1993) the direct attitude towards condom use plays an important part in previous condom use by sexually active adolescents. Further examination of the attitudinal factor shows that sexually active adolescents who always have used con- 
doms, report more positive beliefs about planning and buying condoms and they also claim to be more positive about the costs of condoms than those adolescents who irregularly or never have used condoms. In addition, pupils who irregularly have used condoms are more strongly convinced that condoms disrupt sexual pleasure than those who always or never have used condoms. Yet, in regression analysis the males' intention to use condoms is mainly predicted by determinants, such as (1) the perceived effectiveness of condoms; (2) beliefs about the necessity of using condoms when having sexual intercourse with several partners; (3) the belief that one has to use condoms with an STD; (4) the perception that others find it important to use condoms; (5) previous condom use and (6) the perceived disruptive effect of condoms. The female's intention to use condoms, however, is mainly explained by the perceived importance of using condoms. In addition, the fernale's intention is not influenced by any other belief. Similar to the findings of Varnhagen et al. (1991) beliefs about the planning, the purchase and the costs of condoms do not play any part in both males' and females' intention to use condoms.

In summary, these findings suggest that the attitude towards condom use plays a part in previous condom use and also in intentions to use them in the near future. Nevertheless, previous condom use shows a relationship with factors concerning barriers to use condoms, while these barriers are not related to the intention to use condoms. Furthermore, we may conclude from the above results that different beliefs play a role in the male's and female's intention to use condoms with a casual sexual partner.

Detailed examination of the results regarding the attitude toward condom use among heterosexuals with an HIV testrequest reveals that individuals who have not used condoms are more likely than individuals who have used condoms (1) to find the 'emotional' price of using condoms too high; (2) to regard condom use as a diminisher of sexual pleasure; (3) to evaluate condoms as a sign of mistrust; (4) to perceive difficulties in behaviours related to condom use and (5) to report STD/HIV protective communication as very annoying. Yet, regression analysis showed that of all these attitudinal aspects only two beliefs (regarding condom use as a positive act and evaluating condom use as a sign of distrust) play a rather small and positive role in intention to use condoms with a casual partner.

In summary, these results show that beliefs about personal consequences of using condoms are indeed related to previous condom use, but of all these attitudinal aspects the following two determinants, regarding condom use as a positive act, and evaluating its use as a sign of distrust, play a significant and positive role in intended condon use.

The study on the attitude towards more genera! STD-preventive actions among adolescents reveals on the one hand that sexually inactive adolescents who find talking about STDs important, those who do not find selective partner choice 
annoying, women more likely than men, those who do not find talking about STDs annoying and those who find selective partner choice important are more likely to take STD-preventive actions in the near future than sexually inactive adolescents who do not hold these beliefs. Factors, such as perceived importance and perceived annoyance of condom use do not contribute to the explanation of the intention to perform STD-preventive actions. On the other hand, (besides previous condom use) sexually active adolescents who find selective partner choice and the use of condoms important, those who do not find talking about STDs annoying, women more likely than men, and those who find talking about STDs important, are more likely to perform STD-preventive actions in the near future than sexually active adolescents who do not hold these beliefs.

From the findings on more general STD-preventive actions we may conclude that the following factors explain intended STD-preventive actions: attitudinal aspects of partner choice, attitudinal aspects of STD-protective communication and gender.

\section{Existing akllis and behavioural Intentions}

\section{Previous sexual behaviour and Intentlon to use condoms}

Just over half of the 270 adolescents have had sexual intercourse once or several times during the past year. The majority of these sexually active adolescents never or irregularly have used condoms. A national survey among Dutch adolescents (Vogels \& Van der Vliet, 1990) and other research (Delil, 1988; Richard et al., 1991; Schaalma et al., 1993) and research in the USA (Gottlieb et al., 1988; Strunin \& Hingson, 1987; Kegeles et al., 1988) support this finding. About two-thirds of the sexually active adolescents intend to use condoms in the near future when having sexual intercourse with a new/casual partner. In line with other researchers (Richard et al., 1991; Schaalma et al., 1993; Kegeles et al., 1988) previous condom use proves to be an important predictor of intended condom use. No relationship exists between the number of sexual partners (mutually monogamous versus polygamous relationship) and previous condom use.

The study among 149 heterosexuals with an HIV testrequest shows that a large part of these individuals performed high-risk sexual behaviour, such as the frequent reporting of multiple (anonymous) sexual partners combined with infrequent condom use. The fact that about one-third of this sample has already had an STD before, affirms the statement that heterosexuals with an HIV request are at risk of contracting STDs. About three-quarters of the persons with an HIV testrequest have used condoms irregularly or never during sexual intercourse with casual partners. About half of these heterosexuals state they will definitely use condoms in the near future. Contrary to the findings among adolescents previous condom use was not related to intentions to use condoms during 
sexual intercourse with casual partners among the sample of persons with an HIV testrequest.

The above findings suggest that a large percentage of both groups have shown risk behaviour which could have made transmission of STDs and HIV-infection possible. We may also conclude that condom use is not a regular practice among both Dutch adolescents and heterosexuals with an HIV testrequest. Therefore, both groups of respondents form target groups for STD-related interventions. Furthermore, it appears that previous condom use (habits) influences the adolescent's intention to use condoms with a new partner positively. This finding may reflect behaviour stability over time. Yet, previous condom use among heterosexuals with an HIV testrequest does not explain their intended condom use. One explanation for this discrepancy is that these heterosexuals overestimate their intentions due to the stressful situation in which they completed the questionnaire.

\section{ENABLINO FACTORS AS DETERMINANTS OF STD-PREVENTIVE BEHAVIOUR}

\section{Self-efficacy expectations}

\section{Seli-offlicacy}

In accordance with others (Schaalma et al., 1993; Kegeles et al., 1989; BasenEngquist, 1992), in our study among heterosexuals with an HIV testrequest the concept of self-efficacy expectations plays an important positive part in intended condom use with a casual sexual partner. Further examination of self-efficacy expectations reveals that those who report a low level of self-efficacy expectations are more likely than those who report a high level of self-efficacy expectations to (1) consider condoms as a sign of distrust; (2) perceive STD/HIV protective communication as annoying; (3) report practical problems of using condoms and (4) notice barriers concerning behaviours related to condom use. Those with a high level of self-efficacy expectations are more likely to consider themselves to have a smaller chance of getting HIV infected than peers or friends, regard condom use as a positive act, and show an internal Health Locus of Control than those with a low level of self-efficacy expectations. Yet, in a stepwise multiple regression analysis of these factors only two determinants, perceiving condoms as a sign of distrust and reporting an optimistic infection risk, explain a rather small part (18\%) of the variance in self-efficacy expectations.

To summarise, the findings show that self-efficacy expectation is an important determinant of intended condom use. The exannination of determinants of selfefficacy expectations in turn reveals that although some beliefs about condom use are related to self-efficacy in a multivariate model, only beliefs such as perceiving condoms as a sign of distrust and reporting an optimistic infection risk explain some variance in self-efficacy expectations. 


\section{REINFOACINO FACTORS AS DETERMINANTS OF STD-PAEVENTIVE BEHAVIOUA}

\section{Soclal support and peer Influence}

\section{Social Influence}

In the study among sexually active adolescents the factor perceived beliefs of significant others (parents and friends) about using condorns has a small but negative influence on the male's intention to use condoms $(r=-19 ; p<.05)$, while this determinant plays no part in the female's intention to use condoms. In contrast, other research showed that social norms play a small but positive role (Schaalma ot al., 1993) or did not play a role in intended condom use (Richard et al., 1991; Catania ot al., 1989), respectively.

From these findings we may conclude that our data are partly in contrast with the findings of others. The finding that the male's intended condom use is influenced negatively by social influences of their parents may be explained because the sexual activities may take place despite possibly restrictive beliefs of their parents and because of the adolescents' tendency to become more independent of adults, especially of their parents. In addition, a different operationalisation of the variable 'social influence' may also be the cause of the discrepancies found here. In our research social influence is measured according Ajzen and Fishbein's (1980) prescriptions, namely by normative beliefs multiplied by normative evaluations and then summed up to the variable social influence. We may also conclude that our research findings and the findings of othersi', suggest that the role of social influence on intended condom use is rather vague.

\subsubsection{Determinants of early STD detection behaviour}

The third part of this thesis concerns coping patterns when an STD might have been contracted. With the aim of answering the second question: "What factors are related to behaviours aimed at early detection of Sexually Transmitted Diseases?", three studies among persons with an STD request are discussed here. Firstly, a summary of major findings on determinants of STD self-examination (self diagnosis) and periodic clinical examination are described (see Chapter 6; Leenaars, Kok \& Rombouts, submitted for publication). Secondly, attention is given to the determinants of patients' delay of persons with an STDrequest (see Chapter 7; Leenaars, Rombouts \& Kok, 1993). In this paragraph we will present the results per behaviour under investgation instead of the ordering according to Predisposing, Enabling and Reinforcing factors.

\section{STD self-examination}

From the study among persons with an STD request it may be concluded that the frequency of STD self-examination is not a regular practice at all, while after 
the consultation the majority of the respondents intend to examine their genitals for STD-related symptoms regularly. The intention to STD self-examination is mainly explained by the direct attitude (perceived importance and annoyance) toward STD self-examination as well as the past performance of this recommended behaviour. These findings have already been assessed on other health behaviours (Ajzen \& Fishbein, 1980). Furthermore, we have found that factors from the HBM model such as perceptions of severity and vulnerability do not contribute to the explanation of intented STD self-examination. These results are supported by other studies on self-examination behaviours (Champion, 1985; Massey, 1986). Remarkably, no relationship exists between risk behaviour (unprotected sex) and intention to perform STD self-examination.

In summary, we may conclude from these findings that the performance of STD self-examination is not widespread among persons with an STD request. In addition, the Health Belief Model components, vuinerability and severity, play no role in the frequency of STD self-examination. Unfortunately, benefits of and berriers to STD self-examination have not been measured in this study.

\section{Periodic clinical examination}

The study among persons with an STD request shows that irrespective of prostitution, females score significantly higher than males on the intention to undergo periodic clinical examination. Factors from the HBM model, such as perceived vulnerability and perceived severity account for some of the variance in intended periodic clinical examination. The contribution of these determinants fade in comparison to the contributions of the direct attitude (perceived importance and annoyance) towards periodic clinical examinations which account for most of the variance in the intention to undergo periodic clinical examination. Interestingly, the barriers and benefits index (as measured by negative and positive service attributes of STD health care) do not act as benefits of and barriers to periodic clinical exams at all. Furthermore, it is remarkable that no relationship exists between risk behaviour and intention to seek periodic clinical examination.

From these findings it can be stated that the Health Belief Model components vulnerability and severity have a rather small and positive influence on intention to undergo periodic clinical examination, while benefits of and barriers to medical assessment do not influence the intention to seek periodic clinical examination at all.

\section{Prompt help-seekling behavlour and patiente' delay}

The study among persons with an STD request shows that about one-quarter of the persons with STD-related symptoms delay more than four weeks belore seeking medical assessment. Wornen, especially those with a middle educational background and those who cohabite with one regular partner show long 
delay-behaviour. The delay-behaviour shown by respondents with high-risk sexual behaviour is equal to that of respondents with low-risk sexual behaviour. Villagers, particularly men, are more likely to show delay-behaviour compared to persons living in small and medium-sized towns, and in big cities. While embarrassment and seeking the right physician are more often mentioned by these subjects as reasons for their delay-behaviour, these factors only lead to two weeks' delay-behaviour. Village inhabitants also more often relate consequences of the STD request for their relationships. In addition, heterosexual men and persons who 'know' their sexual partners are more likely to delay than homosexual men and persons who have had sex with anonymous partners. Finally, despite their experience with STD health care, recurrent attenders with STD-related symptoms do not seek medical assessment sooner than those who seek treatment for the first time.

Despite the overwhelming attention paid to sexual behaviour, HIV infection and other STDs, embarrassment and shame about having an STD are still present in the Dutch population and can be seen as barriers to seeking prompt medical assessment for at least some individuals. From the sixteen service attributes only expectations about the existence of walk-in consulting hours, being prescribed proper medication and being cured quickly are more likely to result in prompt help-seeking behaviour.

The four delay-behaviour groups differ from each other with regard to (1) beliefs about anticipated infertility and anticipated skin-injury; (2) the lack of need for a consultation; (3) the attribution of STD-related symptoms to specific sexual behaviour; (4) partner pressure; (5) having received information from mass media; (6) and expectations about the existence of the following services attributes: quick cure, being prescribed proper medication and walk-in consulting hours. Furthermore, personal characteristics such as habits in delay behaviour, shame/embarrassment about STDS and seeking the right physician are also related to delay-behaviour. However, discriminant analysis among two extreme delay-behaviour groups (one week versus more than four weeks' delay-behaviour) reveals that the predictive power of these variables is rather small.

In summary, a relative large part of the persons with an STD request shows delay behaviour. Some variance in delay behaviour is explained by the following determinants: lack of need for consultation, symplom attribution to sexual behaviour, recidence, gender, anticipated skin-injury, partner pressure, and expectations of being prescribed proper medication. 


\subsection{The role of service attributes in the choice of circuits}

In order to answer the third question: "What is the role of specific service attributes (characteristics of health care services) in the choice of STD health care facilities?", the results of two studies among adolescents and persons with an STD request have been taken into account. Firstly, the most important findings of the study on service attributes among persons with an STD request are presented (see Chapter 8; Leenaars, Rombouts \& Kok, 1993). Secondly, the role of service attributes in the intention to choose STD health care providers among adolescents is described (see Chapter 9; Leenaars, Rombouts, Lammerdin \& Zwetsloot, 1989).

\section{Cholce of circults among persone with an STD request}

The study among 825 persons with an STD request shows that special STD clinics are not well known among those visiting their General Practitioner $(\mathrm{N}=108)$ or a Family Planning Clinic $(\mathrm{N}=90)$ for a suspected STD. In the following we compared beliefs about service attributes of STD clinic attenders with beliefs of those visiting a Family Planning Clinic and beliefs of those visiting their GP. The results indicate on the one hand that STD clinics distinguish themselves positively from general practitioners and Family Planning Clinics in the following service attributes: professional treatment, proper medication, GP not notified of the STD examination, the insurance company not notified of the STD examination, free treatment, walk-in consultations and rapid cure. Most of the time, STD clinic attenders attach more importance to these attributes than the two other groups of respondents. On the other hand, STD clinics distinguish themselves negatively from Family Planning Clinics and GPs concerning time spent travelling, interrupting work, waiting time, personal treatment and the possibility of treatment after working hours. The attitude towards these service attributes and social norms about seeking medical treatment at specific health care facilities explain $24 \%$ of the variance in scores between the three choice behaviour groups. It also appears that perceived unprofessionality and embarrassment are important reasons for not asking a GP for medical treatment and for not being loyal to their GPs after a first visit.

To summarise, we may conclude that STD clinics have special service attributes which have been perceived as very important to STD clinic attenders, such as the high quality of medical care, anonymous treatment facilities and walk-in consulting hours. STD clinics also have some negative service attributes, such as the absence of evening consulting hours, the lack of personal treatment and long waiting times. 


\section{Cholce of clrcults among adolescents}

The investigation among adolesents shows that the more specialised institutions such as MHSs and low-threshold out-patient STD clinics are virtually unknown among adolescents. When these pupils suspect they have contracted an STD, most of them plan to go and see their general practitioner and a small percentage intend to go to a more specialised institution.

Hereafter, we compare expectations about the existence of service attributes of those intending to visit a special institution (STD specialists) with those intending to visit a Family Planning Clinic and those visiting their GP. With regard to sixteen service attributes, the Family Planning Clinics distinguish themselves positively from 'STD specialists' and the GP concerning the following service attributes: the STD examination stays unknown to family and friends, the STD examination stays unknown to their GP, the STD examination stays unknown to the medical insurance company, and the option of a male or female physician. Most of the time, adolescents who plan to see a Family Planning Clinic attach more importance to these attributes than the two other groups of respondents.

In summary, adolescents are badly informed about the existence of special STD clinics in the Netherlands, while these STD clinics have important service attributes. In addition, service attributes concerning the taboo on STDs, such as anonymous treatment facilities play an important role in the choice of STD health services. Furthermore, the existence of evening and weekend consultating hours seems to be an important service attribute in STD health care.

\subsection{Methodological issues}

Thus far, we have presented the most important findings of the separate studies. Before presenting recommendations for the content of STD health education and the organisation of STD health care, some limitations of the studies (\$2.2.1) and methodological remarks (\$2.2.2) should be discussed. The main discussion points of the studies are: (1) the generalisability; (2) the problem of causality, (3) self-report measures, (4) the operationalisation of specific variables and (5) the complexity of the behaviours under investigated.

\subsubsection{LImitations of the studles}

\section{The generallsability}

The sample of adolescents consists of 270 pupils from thirteen schools in The Hague. Whether our findings about sexual behaviour among adolescents in The Hague (urban area) can be generalised to secondary school children in other regions of the Netherlands may be a point of discussion. Yet, there is no reason to suspect that we have a select sample of adolescents. When comparing our study with large scale studies among Dutch adolescents Nogels \& Van der 
Vliet, 1990; Richard et al., 1990; Schaalma et al., 1993) we may conclude that our results on determinants of condom use are comparable with findings of these researchers. It should also be mentioned that the potential influence of regional differences on, for example, the availability of STD health care facilities has now been eliminated. Another important advantage of small-scale studies is that a more detailed questionnaire can be administered. Because the study among adolescents was focused on gaining more insight into the determinants of two specific behaviours (preventive behaviour and choice of STD health providers) we have opted for an extensive and detailed survey. In this case a large research group is not as necessary for answering these central questions. The study among heterosexuals with an HIV testrequest consists of 149 persons who attended MHSs or STD clinics in thirteen regions (major cities) in the Netherlands. Those persons who attended their GP or other health care providers did not enter the study. It is estimated, however, that over half of all HIV tests for clinical purposes are requested for by GPs in the Netherlands (Van Lierop, Houweling, Wiessing \& Katchaki, 1992). Yet, it is also generally assumed that persons seeking an STD examination at STD clinics are members of high risk groups (Nan de Laar et al., 1990). From this perspective we may conclude that we have a selected sample of heterosexuals requesting an HIV test, but we also have to note that these specific individuals form an important target group for STD/HIV-related interventions.

Finally, because the written questionnaires were in Dutch, individuals who were not proficient in Dutch were excluded from these studies. Due to the fact that ethnic minority groups represent a relatively large part of the Dutch population and are overrepresented in figures of gonorrhea and syphilis, the results obtained are not representative of the ethnic population in the Netherlands. When a Dutch questionnaire is used, however, this problem exists in most studies on the sexual behaviour of ethnic minority groups. Because of the cultural differences about the meaning of several items on sexual behaviour, simply translating a Dutch questionnaire does not solve the problem.

Crose-sectional character of the studles and causality

Because of the explorative character of the studies presented, definite conclusions about the direction of causality cannot be drawn. Above alli, previous STDpreventive behaviour is 'predicted' by psychological variables measured after the behaviour has occurred. Surveys adininistered only once, however, leave open the possibility that the beliefs expressed by the respondents are post hoc rationalisations for their behaviour rather than a cause of that behaviour. With the aim of validating the predictive utility of the variables assessed in this study a longitudinal design is needed to determine if beliefs ultimately lead to behavioural change. 
Another limitation in the methods of research is especially expressed in the analyses into the influence of service attributes on the intention to choice of STD health services. We have asked the respondents to indicate to what extent they expect a number of service attributes to be available with the institution/provider of their choice, but not to what extent they expect these same features to be available with other providers. As a result of the research method chosen, no definite answer can be given about the contrast between the three subgroups and there is scope for alternative explanations.

\subsubsection{Methodological remarks}

\section{Self-report measures}

The studies relied solely on peoples' self-report measures, which may be subject to problems of response bias. These reports may not be entirely accurate (e.g. over- and underreporting). Moreover, we should ask ourselves to what extent the self-report on sexual behaviour yields socially desirable answers. Yet, the influence of the potential over- or underreporting of, for instance, condom use by adolescents cannot be estimated.

In addition, the majority of adolescents perceive AIDS as a very severe disease; they also know that in order to prevent HIV transmission they have to use condoms with sexual intercourse. In our studies and also in others, socially desirable answers may be expected when asking about intentions to use condoms.

\section{The operationalisation of varlables}

In parts II and III the operationalisation of different variables has been described in detail. Here attention is paid only to some specific variables which have been operationalised in a slightly different way from what is generally supposed to be done.

The expected aversiveness of a negative health outcome is generally discussed in terms of the perceived severity in the HBM. In this study we developed a Perceived Severity Scale to assess perceived severity of ten possible negative consequences for the individual resulting from having an STD. For example: 'If I have an STD, then my regular partner may decide to leave me' and 'If I have an STD, I will feel dirty'. In other research perceived severity was operationalised differently, such as: 'the thought of breast cancer scares me' and 'breast cancer would endanger my life' (Champion, 1990).

Perceived vulnerability is generally assumed to reflect beliefs about the likelihood that a negative health outcome will occur by performing the undesirable behaviour (in this case contracting an STD in the absence of condom use). Consequently, perceived vulnerability is mostly measured as follows: 'I feel I will get breast cancer in the future' or 'I am more likely than the average woman to 
get breast cancer' (Champion, 1990). In this study, however, a Perceived Invulnerability Scale was developed to assess perceived invulnerability to contracting STDs due to nine possible (ineffective) risk reduction behaviours. For example, I thought I had a low risk of contracting an STD, because I/my sexual partner use oral contraceptives' or 'I thought I had a low risk of contracting an STD because I always use condoms'. Mostly, perceived vulnerbility and perceived severity too are measured with one or two items.

To conclude, we have measured some components of the Health Belief Model slightly different from others in this study, because we wanted to find out the specific beliefs behind perceived invulnerability and perceived severity.

\section{The complexity of condom use}

Sexuality is about physical intimacy, love, closeness, excitement, giving oneself to another person etc. In the midst of all this condom use requires people to be sensible and make rational decisions. At that moment they must take a condom, open it, put it correctly on the penis and not forget to hold the rim when pulling out after the act. In addition, condoms are needed most when they are most difficult to use, that is, in a new relationship or in a casual contact. All these situations typically require openness about sex, honesty, responsibility, and some assertiveness. In addition, condom use typically involves verbal negotiation between two people, including: broaching the topic of prophylaxis, dealing with defensiveness or refusal to use them, identifying and negotiating alternatives, how to exit from unsafe sexual situations, and empathy and assertiveness in reaching agreement. For this reason researchers (Darrow \& Pauli, 1985) suggest that both partners in a sexual relationship need to be studied, with special attention to factors that affect communication patterns. In this study only the behaviour of the respondents themselves and their expectations about behavioural consequences have been investigated. This means that no conclusions can be drawn about interactional processes among sexual partners, while our opinion is that not only the respondent's behaviour but also the reaction of a sexual partner ultimately leads to adequate condom use. These considerations have to be taken into account when going over the results from the different studies.

Besides these emotional and communicational aspects concerning condom use, a number of other behavioural patterns may influence actual condom use. These behaviours can be grouped as follows: familiarity with desired type of condoms to be used and distribution channels, buying condoms, and bringing condoms along regularly. With respect to these separate behaviours different determinants may influence their adequate performance. Furthermore, a distinction should also be made in increasing and maintaining regular condom use. At the same time a full identification of any behaviour requires consideration of four elements: action, target, context and time (Ajzen \& Fishbein, 1980). 
In our studies condom use has been operationalised in terms of increasing its use. Condom use has also been viewed at a more general level, namely a generalisation is made over time by using the word 'always'; also with regard to the target behaviour, that is, no specification is made about using, for example, a latex or non-latex condom. The context in our studies is specified by using condoms with a casual partner and the action is formulated in terms of increasing condom use.

The aforementioned methodological problems lead us to the conclusion that the results have to be regarded with a certain degree of caution.

\subsection{Discussion and recommendations}

In order to answer the fourth question of this thesis some recommendations will be presented in this section. The implications of the above results for STDrelated interventions deal with three different aspects: STD prevention (\$ 2.3.1), early detection of STDs (\$ 2.3.2) and the importance of special service attributes in STD health care (\$2.3.3). These aspects will be presented below.

\subsubsection{STD prevention: education and promotion of STD-pre- ventive behaviour}

Many individuals know the basic facts about STDs, yet most do not practice safe sex. In order to prevent STD and HIV infection, proper and consistent condom use has to be promoted. The results mentioned in $\$ 2.1 .1$ showed that there may be different reasons for different target groups to alter their sexual behaviour. There are also different ways in which individuals can be motivated to change their behaviour. Apart from education, facilities and regulation might be necessary conditions for the development of adequate STD control strategies. In this paragraph we will only focus on STD-prevention education.

From our results on primary prevention of STDs and HIV infection two target groups for STD-related interventions are distinguished, namely adolescents and heterosexuals with an HIV request.

\section{The adolescent population as a target group}

Our results, and that of others, suggest that adolescence is a critical period in which to intervene to promote STD-preventive behaviours. As previous condom use (habits) plays an important part in intended condom use with a casual partner, STD prevention education should start before adolescents begin to experiment with sexuality. Because the onset of sexual intercourse occurs at a relatively young age (Nogels \& Van der Vliet, 1990; Schaalma et al., 1993), this 
means that prevention programmes need to be directed to young adolescents as well.

STD prevention education should be aimed primarily at making the adolescents aware of the fact that they should take care of and are responsible for their own sexual behaviour. Consequently, it seems most important to teach adolescents to cope with sexuality in an healthy manner, instead of forbidding them to have sex. In addition, they need to learn some specific behaviours that may lead to prevention of STDs, including HIV-infection, such as:

- making adolescents aware of the fact that they themselves have to decide when and under what circumstances they may initiate sexual activity.

- always using condoms when having sexual intercourse with multiple sex partners or with a new sex partner;

- identifying alternatives for unprotected sexual intercourse, such as petting only or mutual masturbation;

The adolescents' tendency to view condoms primarily as a contraceptive device rather than as an STD prophylactic has already been described by several researchers (Vogels \& Van der Vliet, 1990; Richard et al., 1991; Kegeles et al., 1989). Because of the necessity of condom use among adolescents who have sexual intercourse with multiple permanent partners (serial monogamy) we have made no distinction between those who use condoms for STD prevention and those who use condoms for preginancy prophylaxis.

The results of the study among adolescents suggest that different groups of adolescents need to have different issues addressed in an intervention programme. For example, the results clearly indicate that different programmes for sexually active male and for sexually active female adolescents are required. This idea is virtually in line with the research findings of Schaalma et al. (1993) and Richard ot al. (1991). Detailed examination of the response to individual items also identified specific issues that should be addressed in these two intervention groups.

\section{STD prevention education}

According to our results it is advisable to adress different themes in interventions for sexually active male and female adolescents. For example, it is evident that the following items should be discussed with sexually active males:

- the effectiveness of condom use in preventing STD transmission;

- the importance of condiom use when having sex with different partners;

- the importance of condom use with STDs:

- beliefs about the disruptive effect of condom use.

Apparently, it should be stressed that condoms effectively reduce the transmission of many STDs.

In addition, our study showed that the male's intention to use condoms is likely to be influenced negatively by perceptions of significant others about using 
thern. The idea that the sexual behaviour takes place despite the possibly restrictive beliefs of parents may be an important factor here. This line of reasoning is supported by the idea that a more general tendency is visible during adolescence when adolescents becorne more independent of their parents. In this special case it also seerns advisable to inform parents about possible reactions to their interference with the sexual behaviour of their children. Furthermore, more research has to be done to establish the precise role of the social influence on condom use in this age group.

The intention of sexually active females to use condoms is explained partly by perceived importance to use condoms. This means that other factors play a role in the females" intention to use condoms. Therefore, it is advisable that other possible salient beliefs about condom use (e.g. sign of distrust, embarrassment, dirtiness, their use interfering with spontaneous sex, etc.) than those investigalted in our studies, should also be taken into consideration in future research among fernale adolescents.

Attitudinal aspects of condom use (most barriers) explain previous condom use of adolescents, while more rational expectations about their necessity in different situations and perceived effectiveness influence intended condom use. In accordance with Fishbein and Ajzen's model (1975) non-motivational factors, representing the actual control over behaviour (Ajzen \& Madden, 1986) and which, in this study, include barriers to regular condom use, may also influence the degree of success of actual condom use after the intention is made. As above, previous condom use explains a large part of the variance in the intention to use condoms, indicating behaviour stability over time. It is conceivable that the feedback from previous condom use contains the more positive aspects of condom use. Furthermore, it seems necessary to obtain more information about the consistency of the influence of these barriers to condom use over time.

The strongest but least illuminating associations to intentions to perform STDpreventive behaviours among adolescents, such as condom use, appear to be virtually tautological: regular condom users are best characterised by their strong affirmation about the necessity for condom use. On the surface such findings are not very helpful for health promotion planners. Of more interest are the determinants of the development of such attitudes. Future research should consider possible determinants of this perceived importance. With the aim of influencing the adolescents' direct attitude towards condom use it may be advisable to focus on short term positive consequences of condom use. Therefore, it is advisable to stress during discussion specific short-term advantages of condom use, such as the idea of being optimally protected, viewing condoms as a part of the modern way of life or socially approved behaviour, feeling safe and responsible, and prevention of STDs and HIV infection. 
Perceptions about the importance and annoyance of selective partner choice play a significant part in intention to perform STD-preventive behaviours. This finding suggests that adolescents might view selective partner choice as a remedy for STD prevention and as an alternative for condom use. Yet, selective choice of partners gives no guarantee of freedom from infection, so it is advisable to discuss the beliefs about the effectiveness of this 'preventive' behaviour.

Finally, discussion about these matters with the family and with peers is also mostly absent. For this reason parents should be involved in the set up of prevention programmes in schools. A way of encouraging communication about STDs and condom use is to ask the parents to discuss these matters of sexuality and the importance of prevention of STDs with the adolescents at schools.

\section{Training In communication skills}

Both perceived importance and perceived annoyance of STD communication have a strong influence on intended STD-preventive behaviour. Therefore, these attitudinal aspects of STD communication should be stressed during intervention. Adolescents, however, typically have no appropriate models for negotiating about condom use with sex partners. In film or literature, little or no discussion takes place before the actors and actrices have (unprotected?) sexual intercourse. Therefore, it is not only advisable to present good models on television, but also discuss these matters during the education programme. In addition, it might also be effective to include training in communicative skills, including learning to talk about STDs, learning to negotiate about ways of making love, learning to say no, learning to talk about sexuality. Experience with behaviourally focused interventions has shown that skill-training is successful in risk reduction of unintended pregnancy (Fisher, 1990).

One example of skill-straining is modeling on communication about condom use by using a videotape in which the gradual development of successful interpersonal and communication skills are illustrated. For example, a young woman learning to persuade her partner to use condoms. Another way of learning may be to rehearse specific scenarios with adolescents: for example, asking the persons to walk through how they might broach the topic of condom use with a sexual partner. In addition, discussion might focus on talking about the best time for raising the issue with their partners and encouraging the adolescents to imagine their responses to partner reactions that may range from acceptance to rejection. It also seems advisable to practice these complex behaviours gradually; in other words, suggest a small but steadily increasing number of behavioural changes in the desired direction and offer praise and encouragement with each step. Finally, these scenarios can also be made available in printed form. 


\section{Heterosexuals with an HIV testrequest as a target group}

The majority of persons with an HIV testrequest display sexual behaviour through which transmission of STDs and HIV is possible. We may therefore conclude that persons requesting an HIV test are an important target group for face-to-face interventions. It may be suggested to explore their specific beliefs about condoms and their condom use during pre-and posttest counselling. However, as staked earlier, a majority of these respondents do not like talking about preventive actions during pre- and posttest counselling (Leenaars, Sandfort, Oliehoek \& Schrijvers, in press). Methods used in pre- and posttest counselling may influence these rejective attitudes. It is also conceivable that a possibly moralistic approach towards these heterosexuals has a negative influence on their perceived needs of discussing preventive behaviour.

The results mentioned in \$ 1.2.2 make it clear that several concepts from the theoretical models chosen proved to be determinants of the intention to use condoms, narnely, self-efficacy expectations, perceived threat and some attitudinal aspects of condom use.

\section{Pre- and posttest counselling}

Regarding HIVISTD prevention education specifically, an important aim of preand posttest counselling should be to increase condom use among those who have sexual intercourse with multiple partners.

The results show that condom use may be increased through:

- enhancing self-efficacy expectations;

- discussing the individual's concern about AIDS;

- discussing and encouraging a positive attitude towards condom use.

In order to enhance self-efficacy expectations, the following topics should be talked about during the intervention programme: the belief that condom use is a sign of distrust and the existence of an optimistic infection risk. Consequently, it seems necessary to undermine the belief that condom use is a sign of distrust by focussing mainly on condom use as responsible social behaviour.

The results show that individuals who do report to have an infection risk are: more likely to downplay their infection risk. Such denial of personal risk proved to be greater among young men than young women. Individuals may believe their risk is below average because they sometimes use condoms or believe that they have relatively few sex partners. In addition, because of the stigma associated with AIDS, individuals may deny their own vulnerability. From this we may suggest that the perceived invulnerability observed here emphasises the need to personalise risk appraisal and responsibility in future interventions.

In addition, to encourage self-efficacy expectations among individuals with a low level of self-efficacy one should also tackle attitudinal aspects, such as:

- the annoyance of STD/HIV protective communication (negotiating and talking about STDs); 
- various practical problems of using condoms;

- perceived ease of behaviours related to condom use (such as buying condoms, talking about condom use with a new partner, talking about condom use with a regular partner and putting on a condom).

Like in behaviour therapy, it seems important to enhance self-efficacy expectations not only by entering into discussion, but also by training the skills necessary to perform these separate behaviours. According to Bandura (1986) self-efficacy enhancement may be employed from four sources of self-efficacy information (1) performance attainments based on personal mastery experience (actual condom use); (2) vicarious experience obtained through observation of successful performance of others (e.g. peer leaders may serve as a model); (3) verbal persuasion, and (4) physiological state (e.g. anxiety that the partner will leave) may inform the individual correctly or not that he is capable of performing the behaviour.

Behaviours that need to be learned in order to use condoms adequately may be arranged in a series of steps of increasing difficulty (from buying condoms to using thern). Methods can be found to cope with these matters. Kelley et al. (1989) experimented with such behaviourally focused interventions and showed them to be successful in reducing STO/HIV risk behaviour. Finally, in order to gain more insight into self-efficacy, more research is needed on determinants of self-efficacy about actual condom use as well as on the efficacy of intervention programmes, such as pre-and posttest counselling.

Finally, perceived threat of AIDS explains some of the variance in intended condom use among heterosexuals requesting an HIV test. From this finding we recommend that health promotion efforts should be focused on increasing personal concern about AIDS.

In summary, three components appear to be essential for effective STD/HIV prevention education among adolescents and among heterosexuals requesting an HIV test. Firstly; the programme must provicle relevant information about STDs and HIV infection which allows individuals at risk to both assess their personal risk objectively and to ascertain the best method of risk reduction in their individual social environment.

Secondly, the programme should focus on attitudinal aspects of condom use and STD/HIV protective communication. Special attention should be given to barriers to the recommended behaviours.

Thirdly, the programme should provide training in specific behavioural skills necessary for actually practicing safe sex, such as, how to introduce the topic of safe sex in pre-intercourse discussion, how to negotiate condom use with an unwilling partner and resisting pressures not to use condorns, how to exit from unsafe sexual situations and find alternatives for unprotected sexual intercourse. 
Finally, in the case of HIV testing, especially posttest counselling should be revised to include training in communication skills necessary to negotiate in sexual relationships.

\subsubsection{Early STD detection: education and promotion of early detection behaviour}

Persons seeking medical assessment of an STD or HIV infection have been at risk of infection or at least they perceive themselves to have been so. In addition, many of the individuals attending an STD clinic, or their partners, are members of the so-called 'core groups' of high frequency transmitters of STDs. So, this group of attenders forms a self-selected sample from a general population with regard to individual counselling. Therefore, it seems advisable to see the STD consultation as an opportunity for teaching. An outstanding means to enhance behavioural change and early detection is to add STD education to the treatment. Next, the implications of the results on determinants of STD selfexamination, periodic clinical examination and patients' delay are described in terms of their practical application in STD health care.

\section{STD education durlng consultation}

Although many behavioural messages may be given to patients in the context of STD counselling, in the light of the results on early STD detection four principal messages are discussed here:

1. learn to recognise the major signs and symptoms typical of common STDs and understand that asymptomatic infections are common;

2. promote STD self-examination on signs and symptoms (self-diagnesis) in individuals frequently having unprotected sexual intercourse, with multiple sex partners;

3. pursue prompt medical evaluation of signs and symptoms or after exposure to known or suspected STDs;

4. promote periodic clinical examination in individuals frequently having unprotected sexual intercourse with multiple sex partners.

\section{Recognlse major algns and symptoms}

A disease period typically begins when an individual becornes aware of a change in bodily sensation or feeling state. According to Pennebaker (1982) the recognition of unusual bodily sensations is a function of attention focused on those sensations. The study among persons with an STD request shows that several STD-related symptoms are regarded as normal and therefore people often do not seek medical evaluation immediately. From this finding it seems necessary to provide factual information about STDs to persons seeking a medical examination for a suspected STD. The following key points aimed at 
symptom recognition should be included in the patient-provider contacts: the existence of different types of symptoms showing an STD infection, normal versus abnormal physical symptoms and the existence of asymptomatic STDs. In addition, individuals should recognise (1) that even if their current infection is curable, a future STD may not be; (2) that future infections may be asymptomatic until permanent damage has occurred and (3) that a risk activity for STDs is also a risk activity for HIV infection.

This information should be presented in an explicit form, as rationalisation of symptoms is a device which may prevent self-referral for treatment. Thus, it is also advisable to present this information in pamphlets, brochures and in articles in specific men's or women's magazines. Yet, information alone is not enough to encourage early STD detection. Specific behaviours, such as the performance of regular STD self-examination and to undergo periodic clinical examinations might lead to early detection. Our results show that STD self-examination, periodic clinical examination and prompt help seeking behaviour have their own determinants.

\section{Promote STD self-examination}

Results indicate that STD self-examination is not a regular practice at all and therefore needs to be encouraged, especially among persons who report having frequent unprotected sexual intercourse with multiple sex partners. Moreover, the results suggest that individuals should be taught to consider their sexual behaviour when noticing abnormalities on their genitals or bodily organs such as vagina, penis, mouth and anus. Yet, causal attribution is increasingly subject to interpretive biasses as discomforting sensations increase vagueness, ambiguity and location in parts of the body not directly observable (Pennebaker, 1982). For this reason individuals who have repeated unprotected intercourse with multiple sex partners should be advised that when they are uncertain about vague signs, a medical examination is necessary to determine whether or not the signs indicate an STD. It should also be noted that regular inspection of one's genitals and other bodily organs cannot replace condoms or other safe sex practices.

An important disadvantage of STD self-examination is the fact that the absence of symptoms does not guarantee freedom of infection. Asymptomatic STDs exist and should therefore be taken into account when recommending STD selfexamination. In order to encourage STD self-examination one should mainly focus on the perceived importance and perceived annoyance of self-examination. Positive feedback should also be given to those individuals who already show a habit of self-examination. With the aim of enhancing STD self-examination no priority should be given to themes such as the severity of STDs and vulnerability to STDs. 
Compared with other self-examination behaviours, such as testicular selfexamination, it may be assumed that self-efficacy expectations and behaviour efficacy expectations are important points to discuss here. Another important issue to remind the individuals is to abstain from sexual contacts while symptoms are present or while suspicion of infection exists.

\section{Pursue prompt medical examination of signs}

Once unusual sensations are noticed and recognised as STD-related symptoms they are likely to be reported to a physician. However, $27 \%$ of the sample of persons with STD related symptoms delayed more than four weeks before they sought medical assessment. The findings on patient's delay also suggest that in order to stimulate prompt help seeking behaviour, particularly women and rural inhabitants should be addressed. It seems also meaningful to encourage recurrent attenders to seek prompt medical evaluation when suspecting an STD.

To promote prompt help-seeking behaviour one might start at the STD consultation. The results on determinants of patients' delay show that education about specific themes is advisable. For example, the information should focus on misconceptions, such as washing or cleaning one's genitals after sexual intercourse can prevent one from contracting an STD and the belief that the symptoms will disappear spontaneously. Information should also focus on items such as the need for a consultation when noticing STD-related symptoms or suspecting an STD and the necessity of medical treatment (it possible), the value of having an STD diagnosed quickly and professionally and the possible consequences of untreated STDs, such as infertility and skin-damage. Here again, special attention should paid to the attribution of STD-related symptoms to specific sexual techniques.

Some service attributes play a role in patients' delay. The results suggest that further information about the existence and approach of special STD clinics is necessary. Moreover, publicity about the existence of these special clinics may reduce delay due to seeking adequate medical treatment. More publicity should also be given to special service attributes, such as walk-in consulting hours and professional treatment in STD clinics. In order to promote prompt help seeking behaviour it is also advisable to make use of walk-in consulting hours and physician-designed protocols to ensure proper medication and rapid cure. The absence of any relationship between the other service attributes (e.g. anonymous treatment facilities) and prompt help seeking behaviour may be the result of the overall perceived importance of these special service attributes. This idea of a "ceiling effect" suggests that special service attributes may serve as necessary conditions for STD help-seeking behaviour.

Not only does individual counselling about these matters seem necessary, but also adding these messages to media campaigns, pamphlets and brochures 
and articles in specific men's and women's magazines. It is also advisable to pay special attention to these themes, when educating adolescents about STDs. Another way of encouraging prompt help seeking behaviour is through notitying all sex partners. Partner notification, in this case patient-referred contact tracing, should be encouraged by the health care provider, whereby clear instructions must be given about the importance of warning all sexual partners immediately and motivating them to seek medical treatment promptly.

\section{Promoto porlodic cllnical examinations}

Individuals reporting repeated unprotected sexual imtercourse with multiple sex partners may be motivated to undergo periodic clinical examination of STDs. Especially, when they come in for a consultation, they should be encouraged to come in for STD check-ups on a regular basis. Special attention should be paid to the perceived importance of periodic clinical examination and to perceptions of invulnerability of individuals who report unprotected sexual intercourse with multiple sex partners. The results also show that the availability of special service attributes does not contribute to intentions of periodic clinical examination. Again it may be possible that a 'ceiling effect' has been reached, so the absence of variation is the cause of these results. More research is needed to examine this possibility. It is also important to state that periodic clinical examination cannot replace condoms or other safe sex practices.

\subsubsection{Patlent care: the Importance of special service attributes In STD health care}

In the Netherlands one can obtain medical treatment for a suspected STD at different health care providers, such as: a general practitioner, special STD clinics. Family Planning Clinics, independent specialists and specialists connected to a hospital. Special STD clinics has been developed since 1976, which have free access and provide anonymous medical care to people who are not insured, do not have a GP or wish to remain anonymous because of the taboo on STDs. The results about the importance of service attributes give direction to the devolopment of an adequate STD health care system in the Netherlands.

\section{Acqaintance with STD health care}

Results of both studies on the acquaintance with STD health services suggest that not only adolescents, but also the general public should be supplied with information about the existence and availability of several facilities for curative STD health care in the provinces. Furthermore, it is advisable to stress that socalled STD clinics for curative STD health care do exist. When counselling about the local STD facilities extra attention should especially be paid to adolescents with a non-Dutch cultural background. In addition, information about 
special STD clinics should be given to people now seeking medical treatment from a GP.

\section{Service attrlbutes}

The results of the studies on the importance of service attributes may be useful in making persuasive arguments for the existence of special STD clinics, especially when competing for limited resources. Considering the service attributes where Family Planning Clinics in particular obtain a high score, it goes without saying that STD clinics should take into account such attributes as personal approach and possessing a tolerant attitude towards STD patients when selecting personnel. It is also advisable to hire both male and female physicians and males and females in nursing staff. Both perceived unprofessionality and embarrassment were reasons for not seeking medical treatment from a GP and for not being loyal to their GPs after a first visit. In order to guarantee professional medical treatment it is desirable to work with physiciandesigned protocols adjusted to the practice of GPs.

Regarding the taboo on STDs, it is remarkable that service attributes pertaining to the taboo of STDs, such as anonymous treatment facilities, are still important in STD health care. This means that this desired anonymity should be considered when developing new STD health services. An attempt can be made to avoid contact between STD patients when designing a waiting room and organising operating hours. Most patients visiting an STD clinic did not want their GPs to be informed about nor receive the results of the STD examination. The general practitioner should therefore not be informed about the clinic attendance of such persons. Moreover, it should be pointed out that the STD examination will be treated confidential not only by specialists at STD clinics but also by GPs.

Of the determinants of choice of health services none was more important than the existence of evening or weekend consultating hours. Therefore, it is advisa. ble that operating hours should take into consideration the way of life of patients and daily variations in demand of services, such as evening or weekend consultation hours.

Service attributes which are related to long time investment have been perceived as very annoying. Despite the variable and unpredictable volume of patients inherent in walk-in type clinics, waiting time should be greatly reduced by maintaining an efficient flow of patients from the time of entry until they are discharged. Franklin and Stolz (1984) gave excellent ideas through which long and decompensating delays are anticipated and avoided.

Considering this diversity in expectations and demands of potential STD patients it is, according to us, therefore, advisable to strive for a pluriform provision of assistance. By using already existing infrastructures (Family Planning Clinics, MHSs, STD clinics, general practitioners and independent 
dermatologists) in which cooperation and referral patterns are established, the curative prevention of STDs can acquire an effective structure which is reliable and financially responsible.

\subsubsection{Theoretical conclusions and future research}

\section{Theoretical remarke}

Finally, the theoretical models used in these studies deserve some attention. The results of our studies only partly support the usefulness of the variables from the separate social psychological theories.

Several concepts of the Health Belief Model were used in the studies on determinants of early STD detection behaviours. From the results we may conclude that perceived vulnerability, perceived severity and benefits of and barriers to medical assessment are not sufficient to explain the data. In addition, from the findings of the study on patients' delay in STD patients, it has been shown again that perceived vulnerability and perceived severity do not explain delay-behaviour in individuals with STD-related symptoms either. This may be due to the fact that STDs are generally not seen as severe diseases. In addition, the concepts perceived severity and vulnerability may have been defined inadequately. Another reason for the results may be that the questionnaire was completed right after the consultation. Although the patients had not, at that moment, been informed about the ultimate diagnosis (negative or positive) this might have influenced their perceptions of severity and invulnerability and their intentions to perform early STD detection behaviours. From these findings we conclude that the utility of these concepts of the Health Belief Model is limited in explaining the data obtained. Furthermore, while both intention to perform STD self-exarnination and intention to undergo periodic clinical exams were predicted by the subjects' direct attitude (perceived importance and perceived anneyance) no relationhip has been found between beliefs about treatment outcomes and other service attributes and direct attitude to periodic clinical examination.

With respect to the attitudinal approach in explaining health behaviours as proposed by the Theory of Reasoned Action, we may conclude that various beliefs about condom use were related to previous condom use and also to intentions to use condoms. Yet, in a multivariate model with the direct attitude and self-efficacy expectations, their predictive power was rather small in explaining intended condom use with a casual partner. In addition, the findings on the social norrn as a determinant of intentions of adolescents to use condoms was not related to the female's intended condom use, while it predicted the male's intention to use condoms negatively. We already stated that this finding is in sharp contrast with the findings of others who reported no or a slightly positive relationship with intended condom use. There are many reasons 
which may be responsible for our findings, for example, the adolescents' tendency to become independent of adults and the way social norms have been operationalised.

From the observed findings it should be pointed out that the concepts of the Theory of Reasoned Action gave useful insight into the importance of various beliefs about condom use. Yet, the predictive power of these beliefs on intended condom use is small. One explanation could be that we did not measure the most sailient beliets about outcomes of the recommended behaviours. In addition, the results suggest that other factors have to play a role in intended condom use. Yet, according to the Theory of Reasoned Action, these other factors are only considered as modifying factors. Further research is needed to trace the precise role of other factors in explaining condom use.

Of the determinants of intended condom use of heterosexuals with an HIV testrequest none were more important in explaining intended condom use than self-efficacy expectations. We may, therefore, conclude from the data obtained that the role of Bandura's concept of self-efficacy in intended health behaviour is upheld by our findings.

\section{Future research}

Besides these theoretical remarks the following suggestions for future research were made in the different studies.

Athough the results on determinants of condom use with a casual partner yielded important insight into explaining intended condom use, it is clear that a substantial proportion of the intention to use condoms is not explained by these variables. Clearly, the present studies did not examine all possible determinants of intention to use condoms with a casual partner. For example, we did not ask after beliefs about relationships and beliefs about communication processes, when discussing condom use. Moreover, we only measured beliefs of one person and not the behavioural beliefs of their sexual partners. Therefore, future research on STD-preventive behaviours should also consider relational and communicative processes among sexual partners. In addition to this approach, Luykx, Rademakers, Straver, Van der Rijt \& Van Zessen (1991) also stress the importance of the idea that sexual intercourse takes place within an interpersonal context and therefore more interpersonal and interactional models are necessary to describe sexual behaviour. Apart from this assumption, some researchers (Brown, Diclimente \& Reynolds, 1991) came to the conclusion that more insight into maturational and developmental constructs is needed to understand STD-preventive behaviour among adolescents. Moreover, since sexual decision-making occurs in a broader context of a sociocultural system (Darrow \& Siegel, 1990) it seems important to take into account many other factors, such as, stigma-related coping patterns (denial and misconceptions), the interpersonal nature of STD-disease causation, abnormal illness behaviour (venerop- 
hobia versus perceiving a STD as a minor risk of a particular lifestyle), homosexuality and personality problems.

Another important issue is the quantitative methods used in our studies. Qualitative methods are also advisable, especially to enhance ideas and theories (De Vries, Weits, Dijkstra \& Kok, 1992). Consequently, both partners should be interviewed about interpersonal processes at the same time.

Furthermore, the present studies did not examine all possible determinants of intentions to perform early STD detection behaviours. For example: benefits of and barriers to STD self-examination, issues of self-efficacy expectations (Bandura, 1982) and personality traits may also be important in early STD detection behaviour and should be addressed in future research. We therefore recommend that further research on periodic clinical examination as well as on STD self-examination should start with an exploration of sailient beliets (benefits and barriers) related to perceived importance and perceived annoyance of STD selfexamination. Further research is also necessary to assess determinants of delay-behaviour in women, considering symptom processing and taboos on non-monogamous relationships more briefly.

Finally, attitudes to service attributes and social norms explained only $24 \%$ of the variance in scores between the three choice behaviour groups. This means that other factors should play a role in actual choice behaviour. Therefore, further research should take factors into account, such as personality traits, selfefficacy expectations and confidentiality. 


\section{PART II}

\section{STD PREVENTION}




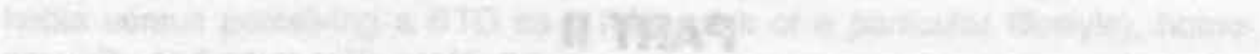

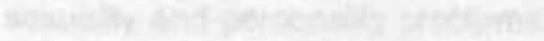

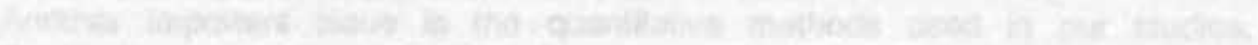

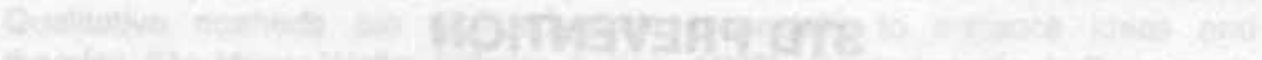
W

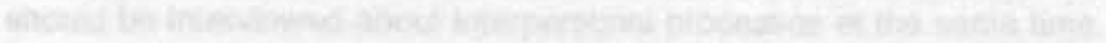

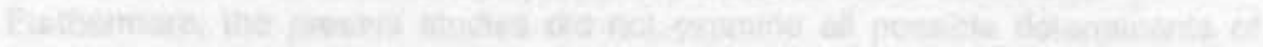

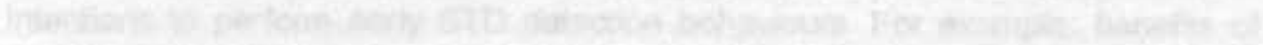

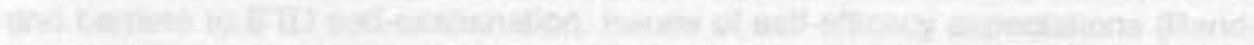

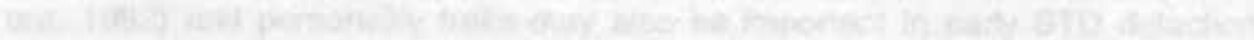
Whation

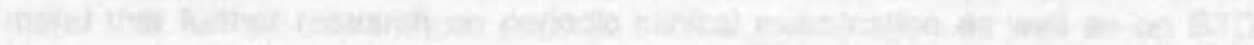

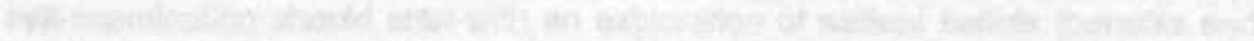

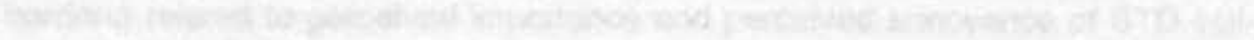

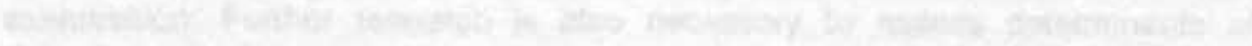

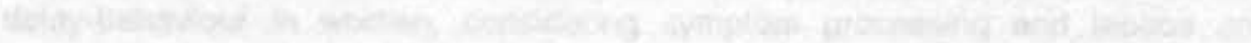

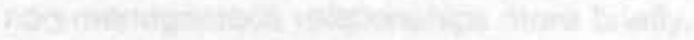

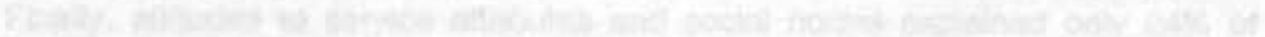

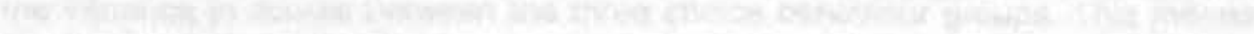

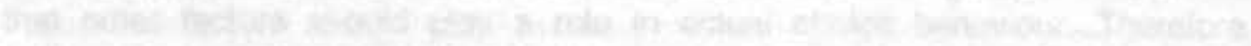

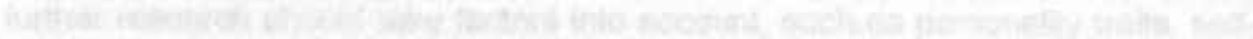

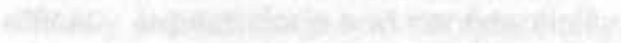




\section{SEXUAL BEHAVIOR AND DETERMINANTS OF CONDOM USE AMONG HETEROSEXUALS REQUESTING AN HIV TEST}

Submitted for publication as: Leenaars P.E.M., Sandfort Th.G.M., Rombouts R., Kok G., Schrijvers A.J.P. Sexual behavior and determinants of condom use among heterosexuals requesting an HIV test.

\subsection{Introduction}

In the Netherlands the Human Immunodeficiency Virus (HIV) is mainly transmitted by traditional risk groups for contracting Sexually Transmitted Diseases (STDs), such as: men with homosexual contacts and injecting drugusers (IDU). Cumulative figures show that as of 1-1-1993 a total of 2678 AIDS cases have been diagnosed in the Netherlands (GHI, 1993). Of the 481 AIDS cases diagnosed in the period $1-1-1992$ to $1-1-1993,87 \%$ belong to the of groups homo/bisexual men $(\mathrm{N}=356)$ and IDU $(\mathrm{N}=60)$. Based on mathematical models regarding the development of the AIDS epidemic, the number of HIV infected persons (including the AIDS patients) was estimated at 9,000-12,000 (of 15 million inhabitants) in the Netherlands as of the first of January 1990 (Jager, Poos, Houweling, Postema, Coutinho, 1990). Although infections as a result of heterosexual contacts are relatively rare, about $10 \%$ of the total population of the diagnosed AIDS cases in 1992 are transmitted heterosexually, a gradual increase in AIDS cases among individuals with heterosexual contacts occured recently (GHI, 1993).

While there is a growing body of information on determinants of STD/HIV. preventive behaviour and behavioural risk factors for contracting an HIV infection among adolescents and homosexual men (the cohor studies), such studies are seldom conducted among heterosexuals with multiple sex partners. The aim of this study is to fill in this gap by assessing the specific sexual behaviour of heterosexuals seeking an HIV test and to investigate delerminants of STD/HIV-preventive behaviours. Ross (1990) stated that specific behaviours, which may be directly related to an increased risk of STD and HIV infection, include psychological concomitants of high numbers of partners, of particular locations of sexual intercourse and of specific sexual practices. Sexual practices that place homosexual men at a particularly high risk for HIV include: unprotected anal intercourse and infections with Herpes virus Nan Griensven, De Vroome, Tielman, 1987; Holmberg, Stewart \& Russell, 1988). Apart from unprotected sexual intercourse with (infected) individuals belonging to high-risk groups, having multiple sex partners and a history of STDs are major risk factors for heterosexual transmission of HIV (CDC, 1988; Greenblatt, Lukehart, Plummer, 1988). Needle sharing and increased blood booting or 'kicking' may 
put especially IDU at increased risk of contracting HIV (Greenfield, Bigelow \& Brooner, 1992).

Besides mutual monogamy and celibacy, condom use is regarded as one of the most effective methods of preventing STD and HIV transmission. Latex condoms are proven to be effective against nearly all STDs, whereas condoms made of sheep intestine (skins) can allow passage of some sexually transmitted diseases and are, therefore, not approved for disease prevention (Lisking, Wharton, Blackburn, 1992). Despite mass media campaigns designed to Increase the publics awareness of HIV/AIDS and to promote 'safer sex', recent increases in homosexually transmitted anorectal and urethral gonorrhea and syphilis among attenders of an STD clinic in Amsterdam were reported Nan den Hoek, Van Haastrecht, Henquet \& Coutinho, 1993). Additionally, recent analysis of behavioural data reported by participants of a cohort of homosexual men in Amsterdam showed an increase in the percentage of men who had unprotected insertive or receptive anogenital intercourse (an increase from $13,1 \%$ to $24.0 \%$ among men with casual partners) (De Wit, Van Griensven, Sandfort \& Coutinho, 1993). These findings are supported by a study among the Dutch population, which showed that a remarkably high percentage persists in unsafe sexual behaviour (Nan Zessen \& Sandfort, 1991). Furthermore, Vogels and Van der Vliet (1990) concluded on the basis of a study among 11,500 secondary school children that condom use seems to be popular; $60 \%$ used a condom during their first sexual encounter and $40 \%$ used a condom during the last time they had sexual intercourse. Nevertheless, the most important motive for using condoms was protection against pregnancy; only $13 \%$ used both the pill and a condom. From the above research findings it seems probable that the preventive messages are not received by these groups, or that the educational message is targeted at the wrong determinants. These findings also stress the need for research on risk behaviours and determinants of condom use among persons who are sexually active with more than one partner.

In order to develop effective HIV prevention strategies for pre- and posttest counselling it is essential to get a better understanding of the high risk sexual behaviours of heterosexuals with multiple sex partners. Additionally, it seems worthwile to gain better insight into determinants of condom use by this specific risk group.

The theoretical perspective of the present study

The Theory of Reasoned Action (Fishbein \& Ajzen, 1975), which has been used successfully to describe contraceptive behaviour (Adler, Kegeles, Erwin \& Wibblesman, 1990), family planning behaviours (Jaccard \& Davidson, 1972) and intention to use condoms (De Wit, Kok, Timmermans \& Wiinsma, 1990; Hickson, Weatherburn, Davies, Hunt, Coxon \& McManus, 1992; McKusick, Coates, Wiley, Morin \& Stall, 1987), underlied this study and guided us in our selection 
of variables. Ajzen and Fishbeins' theory suggests that condom use can be predicted by the saliency of beliefs about the consequences of using or not using condoms (attitude) combined with beliefs about the views of significant others of this use or non-use and the motivation to comply with those individuals (social norms). While social norms may be important to predict condom use, in this study we only focussed on attitudes towards condom use.

Several extentions of this basic model were made and included in this study:

- Bandura's self-efficacy concept (Bandura, 1989), the respondent's belief that one can excert control over motivation, behaviour and social environment, also named perceived behavioural control in the Theory of Planned Behaviour(Ajzen \& Madden, 1986), appeared to be important in several healthrelated behaviours (Bandura, 1989; De Vries, Dijkstra \& Kuhlman, 1988) and specifically in predicting the intention to use condoms among adolescents (Emmons, Joseph, Kessler, Wortman, Montgomery \& Ostrow, 1986).

- Knowledge: it goes without saying that adequate knowledge about (prevention of) HIV transmission is a necessary condition for performing HIV preventive behaviour. Knowledge of the risk factors involved in HIV transmission played a role in motivating initial behavioural change (Emmons et al., 1986. On the other hand, knowledge of HIV transmission behaviours and safe sex practices were not associated with self-reported condom use (Baldwin \& Baldwin, 1988; Keller, Schleiffer, Bartlett, Johnson, 1988) and sexual risk behaviour (Joseph, Montgomery, Emmons, Kessler, Ostrow, Wortman, O'Brien \& Eshleman, 1987).

- Perceptions of personal risk: as a fundamental component of the Health Belief Model, perceived personal risk appears to be more important than objective knowledge in motivating behavioural change (Joseph et al., 1987) It has been noted that heightened perception of risk results in behaviour change, although the effects may diminish over time (Allard, 1989; Becker \& Joseph, 1988; Emmons et al., 1986). Meanwhile, other findings show that many people remain unaware of their own risk of becoming HIV infected (Becker \& Joseph, 1988; Cleary, Rogers, Singer, Avorn, Devanter, Perry \& Pindyck, 1986). Additionally, most research among adolescents shows that adolescents evaluate AIDS as a serious disease, but few see AIDS as a threat to their own health (Baldwin \& Baldwin, 1988; Hingson, Strunin, Berlin \& Heeren, 1990).

- Health Locus of Control (HLC): is one form of the Locus of Control construct derived from the Social Learning Theory of Rotter (Rotter, Chance \& Phares, 1972). Health Locus of Control appeared to be an important determinant of several health behaviours, for example: a relationship existed between internal HLC and compliance (Schlenk \& Hart, 1984). Despite the fact that $H L C$ is sometimes viewed as a personality trait, attempts to change it were 
described by Wallston and Wallston (1987). To our knowledge HLC was not incorporated in other studies on determinants of condom use.

\subsection{Methods}

\section{Subjects and procedure}

Subjects who attended Municipal Health Services (MHSs) or STD clinics in thirteen regions (major cities in the Netherlands) for an HIV test entered the current study. Individuals who were not proficient in Dutch, respondents who sought an HIV test after incest and those who attended a second time because of the window period, were excluded from the study. The health care provider encouraged the individuals to take part in the investigation and then provided them with instructions for answering the questionnaire. All subjects completed the first part of the questionnaire after the pretest consultation and the second part after the posttest consultation. Anonimity was guaranteed.

Apart from the 326 respondents who took part in the investigation, about one out of five persons seeking an HIV test refused to fill in the questionnaire after the pretest consultation. Almost $20 \%$ of the 326 respondents did not complete the second part after the posttest consultation. Reasons for this were not enough time, first part was too long and some of the questions were too personal, did not return for the test result, got the test result by telephone, health care provider forgot to hand out the questionnaire.

No differences were found in age, gender, educational level and sexual preference between respondents who filled in the first and second part of the questionnaire and those who only completed the first part.

Because of the central theme of this study, determinants of condom use with a new/casual sexual partner, the following respondents were excluded from the analyses: (1) those who did not have sexual intercourse in the past year $(\mathrm{N}=14)$; (2) those who did not have sexual intercourse with a casual partner apart from their steacly partner in the past year $(\mathrm{N}=46)$; (3) those who reported sexual intercourse with individuals of the same gender $(N=40)$ and $(4)$ those who were paid for sexual intercourse (prostitutes) $(\mathrm{N}=11)$. Thus, all respondents described in this study are heterosexuals who sought an HIV test because of their own sexual behaviour. A total of 149 respondents were left out for further analyses.

\section{Measures and datareduction}

This study involved the following variables:

- Sociodemographic characteristics: age, gender, education, paid job, frequency of past HIV and STD consultations;

- Sexual behaviour history: five aspects of the participants sexual behaviour history were assessed: (1) total number of different sex partners in the preceding year; (2) sexual preference; (3) sexual intercourse with anony- 
mous partners; (4) sexual intercourse with a prostitute and (5) being a prostitute. Sexual intercourse was defined as vaginal or anal sex.

- Intention to use condoms: the respondents were asked on what level they intend to use condoms every time they have sexual intercourse with a new/casual partner (7-point scale ranging from $1=$ no certainly not to $7=y e s$ very certain).

- Reported infection risk. was rated 'yes' if a person had unprotected vaginal or anal sexual intercourse in or outside a steady relationship during the previous year.

- Estimated infection risk. was measured with one item on a 7-point scale question (1=very small to $7=$ =very large). "How large do you estimate your chance of getting HIV infected in the near future?".

- Optimistic infection risk. measured by two items on a 5-point scale (1=a much larger chance to $5=a$ much smaller chance). For example: if you look at persons of your age do you think that you have a larger, an equal or a smaller chance of becoming infected with HIV? $(r=0.43)$.

- Beliefs about condom use: assessed by 23 beliefs about using condoms. A 5-point scale (1=disagree entirely to $5=$ agree entirely) was used for each belief item. We ultimately selected these 23 beliefs based on a literature study on the contribution of a variety of beliets about condom use in predicting condom use by Dutch adolescents and homosexual men. Factor analysis with varimax rotation resulted in seven components, accounting for $62 \%$ of the variance:

- condoms diminish sexual pleasure, comprising of eight items (e.g. a condom is an annoying interruption when making love), Cronbach's alpha was 0.82 ;

annoyance of STD/HIV protective communication, comprising of two items (e.g. talking about STDs with a new sexual partner is annoying), $\mathrm{r}=0.77$;

condoms as a sign of distrust, comprising of four items (e.g. using a condom is a sign of mistrust), Cronbach's alpha was 0.60 ;

(emotional) price of using condoms, comprising of three items (e.g.: a condom makes you think about AIDS), Cronbach's alpha was 0.53 ;

practical problems of using condoms, comprising of two items (e.g. condoms slip off easily), $r=0.30$;

negative consequences of wanting to use condoms, comprising of two items (e.g.: when I use condoms with a new sexual partner that person may decide against having further intercourse with me), $r=0.28$;

condom use as a positive act, comprising of two items (when I use condoms I protect myself from becoming HIV infected), $r=0.25$.

- Perceived ease of behaviours related to condom use: measured by five items involving five behavioural aspects of condom-use (buying condoms, 
putting on condoms, negotiating with a regular partner, negotiating with a casual/new partner, always using condoms). A 5-point scale with endpoints 'very easy' and very difficult' was used for each item. For example: how difficult do you find talking about condom use with a new partner?, Cronbach's alpha was 0.63 ;

- Knowledge of HIV-transmission: respondents were asked to which degree (3-point scale: entirely out of question, out of question, possible) it is possible for HIV to be transmitted in four situations (kissing, mosquito bite, sexual intercourse with an HIV infected partner, toilets);

- Self-efficacy expectations. measured by three items on a 7-point scale ( $1=n o$, certainly not to $7=y e s$ very certain), assessing self-efficacy expectaltions about using condoms in three different situations (a new partner, a known partner and under the influence of alcohol). For example: do you think you will always succeed in using condoms even when you are under the influence of alcohol? Cronbach's alpha was 0.71:

- Perceived threat of AIDS: measured by two iterns on a 3-point scale ( $1=$ not at all threatening to $3=$ very threatening) respondents were asked how threatening they perceive AIDS for their immediate environment?, $r=0.23$;

- Health Locus of Control: was measured by seven items evaluating Health Locus of Control (HLC). A 7-point scale (1=disagree entirely and 7=agree entirely) was used for each item. A high score indicates an internal HLC, a low score an external HLC (powerful others and chance). For example: it is my own fault if I become HIV infected. Cronbach's alpha for the total scale was 0.71 .

\section{Data analysis}

All data were performed by standard statistical procedures of the SPSS/PC version 3.0 (Norusis, 1988). Multivariate analysis of variance was used to investigate whether respondents who had run a risk of infection in the previous year differ from those who did not report a risk of infection in: HLC, sell-efficacy, benefits of and barriers to condom use, estimated risk of infection and optimistic risk of infection. Univariate analyses of variance and Scheffe multiple comparison tests were carried out to assess relationships between sociodemographic characteristics and intention to use condoms with a new/cassual sexual partners. Pearson cortelations were calculated between the intention to use condoms, with new/casual partners and all aforementioned psychological variables.

Next, to assess the relative predictive power of variables related to intention to use condoms with a casual/now partner a stepwise multivariate regression analysis was carried out on all variables significantly $(p<.05)$ related to the intention to use condoms. To gain more insight into the predicive power of behavioural variables related to self-efficacy expectations; a second stepwise multiple-regression analysis was carried out. 


\subsection{Results}

\section{Description of respondents}

The total group of respondents consisted of 88 men and $61(41 \%)$ women. Half of the respondents were aged between $24-35$ (mean age $=31 ; s . d=9.1$ ). More than half of the respondents $(59 \%)$ had a high educational background (higher vocational education and university), $22 \%$ completed grammar school (pre university education, girls' secondary school), $16 \%$ completed a technical or middle vocational training (a commercial school, advanced elementary school) and the remaining participants (4\%) only completed elementary school. Almost two-thirds have paid employment; of the respondents without paid employment $54 \%$ are students, $25 \%$ are out of work or are incapacitated for work $(14 \%)$ or retired early. Most respondents (95\%) have the Dutch nationality.

\section{Characterlstlc features of sexual behaviour and reported Infection rlsk}

\section{Respondents with a steady relationshlp}

The majority of the individuals (65\%) reported a steady relationship. Almost twothirds of the respondents with a steady relationship are men. The duration of the steady relationships varies from less than a month to over nineteen years and the average duration is two years. The number of different partners (apart from a regular partner) with whom one has had sexual intercourse during the last year varies from one $(40 \%)$ to thirty casual partners. Three-quarters of the participants had sexual intercourse with these casual partners more than once.

Almost one-third of the respondents had sexual intercourse with an unknown partner, $9 \%$ paid for the sexual intercourse and almost one-quarter had sexual intercourse with someone who lives outside the Netherlands. Half of the respondents had a STD examination in the previous year, $25 \%$ had an STD, while $18 \%$ already had an HIV test in the past.

If respondents with a regular partner had vaginal sexual intercourse with casual partners in the preceding year, $38 \%$ never used condoms, $42 \%$ irregularly and $21 \%$ used condoms very often. Eleven heterosexual respondents reported having had anal sex with a casual partner in the preceding year; seven out of these eleven individuals did not use condoms with anal sex. Finally, half of the respondents had unprotected sexual intercourse while they were under influence of alcohol or other drugs.

\section{Respondents without a steady relationshlp}

One-third of the respondents do not have a steady relationship. About threequarters of the respondents without a steady relationship are women. The mean number of different partners with whom they had sexual intercourse during the preceding year is well over four (modus=1 partner; $s . d$. = 8.6.). No difference 
was found between men and women in the number of sexual partners. Almost all respondents had sexual intercourse more than once with these partners. Half of the respondents without a steady relationship had sexual intercourse with an unknown partner in the preceding year, $13 \%$ paid for their sexual intercourse and $31 \%$ had sexual intercourse with a casual partner who lives outside the Netherlands. One-third had an STD examination in the last year, almost onethird was diagnosed positively on STDs, $16 \%$ had an HIV test. Three-quarters of the respondents without a steady relationship never $(26 \%)$ or irreqularly $(48 \%)$ used condoms during vaginal sexual intercourse. Three respondents reported unprotected anal sexual intercourse. Additionally, half of the respondents mentioned unprotected intercourse while they were under the influence of alcohol or drugs.

Reported-, optimistic- and estimated infection riak, and percelved threat Seven out of ten individuals who had sexual intercourse with casual partners during the year prior to the test reported sexual behaviour which could have made transmission of HIV possible. There are no differences in reported infection risk between men and women respectively and between respondents with a regular partner and those with a casual partner. There is also no connection between age and reported infection risk. Finally, respondents who reported an infection risk claimed more casual partners (mean partner number $=2$ to 3 ) than those who reported no infection risk (mean partner number $=1)(F=3.8 ; p<0$ .05).

We assumed that if these respondents estimate their chance of getting HIV Infected smaller than their peers they show an optimistic infection risk. Almost $30 \%$ of the respondents claimed their chance of becoming HIV infected to be smaller than their peers and $16 \%$ of the respondents reported their chance of getting HIV infected to be smaller than that of their friends.

Allmost all respondents estimated their chance of contracting HIV in the near future as small to very small, while $12 \%$ showed ambivalent feelings about becoming HIV infected. It should be noted that these items were stated in unconditional probabilities based on whatever set of factors the individuals took into account.

Half of the respondents evaluated AIDS as very threatening to the Dutch population and only $9 \%$ stated that AIDS is very threatening for their own communities. Nearly all respondents estimated their chance of getting HIV Infected in the near future small to very small and only $12 \%$ showed ambivalent feolings about getting infected with HIV.

In order to gain more insight into differences between respondents whe reportecl an infection risk and those who did not report an infection risk on factors, such as: benefits of and barriers to condom use, self-efficacy, perceived threat, 
knowledge of HIV transmission, optimistic infection risk and estimated infection risk a multivariate analysis of variance was carried out (see Table 4).

TABLE 4. MEAN SCORES AND F-STATISTICS OF BEUEFS ABOUT CONDOM USE, HLC, PERCEIVED THREAT, SELF EFFICACY AND INFECTION AISK

\begin{tabular}{|c|c|c|c|c|c|}
\hline \multirow{2}{*}{ 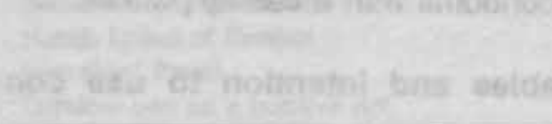 } & \multicolumn{2}{|c|}{$\begin{array}{c}\text { HAVE RUN A } \\
\text { RISK }\end{array}$} & \multicolumn{2}{|c|}{ NO RISK } & \multirow[t]{2}{*}{ F.VAL_UE } \\
\hline & M & S.D. & $M$ & S.D. & \\
\hline (emotional) price of using condoms & 2.1 & 0.7 & 1.8 & 0.7 & $4.2^{*}$ \\
\hline Condoms diminish sexual plessure & 3.3 & 0.6 & 3.0 & 0.6 & $5.0^{\text {th }}$ \\
\hline Practical problems of using condom & 2.4 & 0.8 & 2.2 & 0.7 & 1.1 \\
\hline Condome a sign of distruat & 2.5 & 0.6 & 2.2 & 0.6 & 6. $9^{4}$ \\
\hline Porceived ease of condom use behaviours & 2.8 & 0.5 & 2.5 & 0.6 & $18.3^{*}$ \\
\hline Neg. conseq. of wenting to use condoms & 2.3 & 0.7 & 2.3 & 0.8 & 0.1 \\
\hline Annoyance of STD/HIV protective comm. & 3.0 & 1.1 & 2.6 & 1.0 & $4.5^{\mathrm{th}}$ \\
\hline Condom use as a positive act & 1.8 & 0.5 & 1.8 & 0.4 & 0.0 \\
\hline Health Locus of Control & 2.2 & 0.8 & 2.4 & 0.7 & 0.5 \\
\hline Self-efficacy expectations & 4.3 & 0.7 & 4.8 & 0.7 & $6.8^{*}$ \\
\hline Perceived threat of ADS & 2.1 & 0.4 & 2.1 & 0.4 & 0.3 \\
\hline Knowledge of HIV transmission & 1.8 & 0.4 & 1.7 & 0.4 & 1.3 \\
\hline Estimated infection risk & 2.0 & 1.1 & 1.9 & 1.1 & 1.2 \\
\hline Optimistic infection risk & 3.3 & 0.6 & 2.9 & 0.6 & 4.4* \\
\hline
\end{tabular}

note: * $p<0.05$

Table 4 shows that respondents who reported an infection risk are more likely to claim the (emotional) price of condoms to be too high, they more often state that condoms diminish sexual pleasure, they more often report that condom use is a sign of mistrust, they are more likely to experience problems with specific: behaviours related to condom use, they find discussing about condom use and STDs more often annoying, they are less certain about using condoms in different situations and, finally, they are less likely to state that they have a smaller chance of getting HIV infected than their friends. Despite the aforementioned significant differences the practical meaning of these relatively small differences must be taken into account when discussing results.

\section{Determinants of Intention to use condoms with a casual partner}

\section{Intention to use condoms}

Nineteen out of 149 respondents (13\%) claimed they will not use condoms with a casual partner in the near future, one-third reported they will somehow use a condom and half stated they will definitely use a condom with a casual partner $(M=5.7 ;$;.d. $=1.1)$. 
Rolatlonshlps between sociodemographlc varlables and Intention to use condoms

No relationships existed between gender, educational level and type of sexual relationship on the one hand and intention to use condoms with a casual/nevv partner on the other. Age and number of sexual partners in the previous year were also not related to the intention to use condoms with a casual partner.

\section{Relationehips between behavioural varlables and Intention to use con- doms}

Pearson correlations were calculated between intention to use condoms and beliefs about condom use, perceived threat, HLC, self-efficacy, estimated infection risk and optimistic infection risk and previous condom use. Respondents who intended to use condoms with a casualnew partner were more likely: to report stronger self-efficacy expectations, to consider condom use a positive act, to perceive AIDS as threatening, to show an internal HLC and to claim they have a smaller chance to get HIV infected than peers or friends. Additionally, respondents who claimed a lower intention to use condoms with a casual partner are more likely: to evaluate condom use as a sign of distrust, to perceive STD/HIV protective communication as annoying, to perceive practical problems when using condoms, to perceive difficulties in behaviours related to condom use and to estimate their infection risk in the near future as small (see Table 5). No relationship existed between previous condom use, perceiving condom use as a diminisher of sexual pleasure, the (emotional) price of using condoms, knowledge of HIV transmission on the one hand and intention to use condoms on the other.

\section{Determinants of Interided condom-use}

In order to assess the relative predictive power a stepwise multiple regression analysis of the intention to use condoms with a new/casual partner on all significant related variables with the intention to use condoms was carried out (see Table 5).

Regression analysis of the intention to use condoms with a new/casual partner on the following factors: self-efficacy, condoms as a sign of distrust, annoyance of STD/HIV protective communication, perceived difficulties in behaviours related to condorn use, evaluating condom use as a positive act, estimated infection risk, optimistic infection risk, perceived threat and HLC revealed that the intention to use condoms with a new/casual sex partner was mainly explained by having positive self-efficacy expectations, showing an internal HLC and perceiving AIDS as a threat. In addition, percelving condom use as a positive act and viewing condoms, as sign of mistrust adds $3 \%$ of explained variance in the interition to use condoms. 
TABLE 5. RELATIONSHIPS BETWEEN SELF-EFFICACY, BENEFITS OF AND BARRIERS TO CONDON USE, PERCENED THREAT, ESTIMATED- AND OPTIMISTIC INFECTION RISK, HLC AND INTENTION TO USE CONDOMS

\begin{tabular}{llllll}
\hline & r & part. r & bota & MR \\
\hline Self-eficecy expectations & 0.47 & 0.38 & 0.47 & 0.47 \\
Health Locus of Control & 0.32 & 0.22 & 0.24 & 0.52 \\
Perceived threat & 0.19 & 0.22 & 0.21 & 0.56 \\
Condom use as a positive act & 0.30 & -0.19 & 0.20 & 0.60 \\
Condoms as sign of distrust & -0.39 & -0.17 & 0.19 & 0.62 \\
Annoyance of STD/HIV communication & -0.22 & & & \\
Practical problems of using condoms & -0.17 & & & \\
Nogative consequences of wanting to use condoms & -0.22 & & & \\
Perceived ease of condom use behaviors & -0.22 & & & \\
Estimated infection risk & -0.25 & & & \\
Optimistic infection risk & 0.18 & & & \\
\hline
\end{tabular}

note: all correlations mentioned are significant $(\rho<0.05)$

\section{Self-efficacy expectations and determinants of self-efficacy}

One-quarter of the respondents stated that they are more or less convinced they will use condoms in several different situations $(M=4.5 . ; s . d .=1.0)$. No differences were found in self-efficacy expectations between men and women, low, middle and high educated respondents respectively. In addition, respondents who were at risk for an HIV infection were less convinced about using condoms in different situations $(M=4.3)$ than those who did not run a risk of infection ( $M=4.8)(F=4.5 ; p<.03)$.

table 6. RELATIONSHIPS BETWEEN BENEFTTS OF AND BARAIERS TO CONDOM USE, PERCENED THREAT, ESTIMATED- AND OPTIMISTIC INFECTION RISK, HLC AND SELF-EFFICACY EXPECTATIONS

\begin{tabular}{lrrrrr}
\hline & $r$ & part.r & beta & MR \\
\hline Perceiving condoms as a sign of distrust & -0.35 & 0.36 & -0.35 & 0.36 \\
Optimistic infection risk & 0.24 & 0.42 & 0.19 & 0.42 \\
Annoyance of STD/HIV protective communication & -0.25 & & & \\
Practical problems of using condoms & -0.18 & & & \\
Condom use as a positive act & 0.19 & & \\
Perceived ease of condom use behaviours & 0.29 & & \\
Estimated infection risk & -0.24 & & \\
Optimistic infection risk & -0.24 & & & \\
Health Locus of Control & 0.18 & & & \\
\hline
\end{tabular}

note: alle correlations mentioned are significent $(p<0.05)$. 
Pearson correlations in Table 6 show that respondents with a low level of selfefficacy expectations were more likely: to consider condoms a sign of distrust, to perceive STD/HIV protective communication as annoying, to report practical problems of using condoms and to notice barriers in behaviours related to condom use. Table 6 also shows that respondents with a high level of selfefficacy expectations considered themselves to have a smaller chance of getting HIV infected than their peers, regard condom use as a positive act and show an internal HLC.

A second stepwise regression analysis of self-efficacy expectations on the following variables: condoms as a sign of distrust, annoyance of STD/HIV protective communication, practical problems of using condoms, condom use as a positive act, difficulties of behaviours related to condom use, estimated infection risk, optimistic infection risk and HLC, shows that: perceiving condoms as a sign of distrust and reporting an optimistic infection risk explain $18 \%$ of the variance in sell-efficacy expectations (see Table 6).

\subsection{Discusslon}

The present study focused on a specific group of heterosexuals attending STD clinics and Municipal Health Services for an HIV test. Considering the highly sensitive nature of some of the questions, particularly those on self-reported sexual behaviour and the length of the questionnaire, this study achieved a high response rate of $75 \%$. No differences were found in socio demographic characteristics between respondents and non-respondents.

By only including the research population of the persons who were tested anonymously at MHSs and STD clinics, we can speak of selection, particularly because according to recent research Nan Lierop, Houweling, Wiessing \& Katchaki, 1992) a large part of the HIV tests are carried out through the General Practitioners in accordance with the current policy in the Netherlands. Despite their expected important share in HIV prevalence figures, ethnic minority groups were also excluded from this investigation if they were unable to complete the questionnaire, which was in Dutch.

It is remarkable that half of the individuals requesting an HIV test were highly educated, while among persons seeking an STD examination at MHSs and STD clinics only $23 \%$ reported a high level of education (Leenaars, Rombouts \& Kok, 1993). A possible explanation for this discrepancy is that low educated persons are unacqainted with institutions where one can get an HIV test, such as MHSs and STD clinics. It is also possible that persons with a high education cope differently with HIV risks than those with a low education.

From the results on sexual behavioural characteristics we may conclude that a large part of these heterosexual individuals were indeed at increased risk of contracting HIV and other STDs. Partly consistent with our findings other 
researchers (Chapman, Stoker, Ward, Porritt \& Fahey, 1990) also concluded that among heterosexuals with multiple sex partners, the following attitudinal factors discriminated between condom-users and non-users: beliefs about condom use as a positive action, condom use as a cue to embarrassment and condom use as an antithetical to good sex. In our study we also found that individuals who had not used condoms were more likely than those who had used condoms: to find the (emotional) price of using condoms too high, to regard condom use as a diminisher of sexual pleasure, to evaluate condoms as a sign of distrust, to perceive difficulties in behaviours related to condom use and to report STD/HIV protective communication as very annoying. From these results it seems advisable to explore these specific beliefs about condoms and condom use during pré and posttest counselling.

In agreement with findings of other studies (Baldwin \& Baldwin, 1988; Keller et al., 1988) knowledge about HIV and HIV transmission was not related to intended condom use.

The results also showed that individuals who did not report an infection risk were more likely to downplay their infection risk on the one hand, while they showed a higher level of self-efficacy expectations on the other. Such denial of personal risk is shown to be greater among young men than young women, in particular those who report multiple sexual partners and irregular condom use (Hansen, Hahn \& Wolkenstein, 1990). The existence of an optimistic bias among heterosexuals at relative high risk of an HIV infection (STD clinic attenders) (Bauman \& Siegel, 1987) was about the same $(30 \%)$ as we measured in this population. Bauman and Siegel (1987) also concluded that gay men underestimate the severity of risk-increasing behaviours and overestimate the value of what they perceive to be risk-decreasing behaviours. Individuals may think their risk is below average because they sometimes use condoms or believe they have relatively few sex partners. It could also be argued that since the current prevalence of HIVIAIDS among the heterosexual population is relatively low (GHI, 1993) people's perception of risk may be correct.

In line with previous findings (Bauman \& Siegel, 1987; Richard, Van der Pligt \& De Vries, 1991) in this study self-efficacy expectations play an important role in the intention to use condoms. According to Bandura (1989) translating health knowledge into effective self protection against HIV infection requires social skills and a sense of personal power to exercise control over (interpersonal) sexual relationships.

Despite the fact that several beliefs about condom use were related to selfefficacy expectations, only one variable, perceiving condoms as a sign of distrust, explained a small amount of the variance of self-efficacy expectations in a multivariate model.

Having an internal Health Locus of Control explained some of the variance in intention to use condoms. Despite the view that HLC is regarded as a personali- 
ty trait, attempts to change it have been described by Wallston and Wallston (1987). In this case having an internal Health Locus of Control can be seen as a prerequisite for active STD/HIV preventive behaviour. Finally, some variance in intention to use condoms was explained by perceiving ADS as threatening, regarding condom use as a positive act and perceiving condoms as a sign of mistrust. From these findings we recommend that health promotion efforts should be focussed on increasing personal concern about AIDS. It also seems necessary to undermine the belief that condom use is a sign distrust by focussing mainly on condom use as responsible behaviour. While studies among adolescents (Leenaars, Rombouts, Lammerdin \& Zwetsloot, 1991: Van der velde \& Van der Pligt, 1991) showed that previous condom use predicted intention to use condoms in the near future in this investigation, however, previous behaviour was not related to intention to use condoms. It may be possible that these heterosexuals overestimated their intentions due to the distressing situation in which they answered the questionnaire.

The results of this study only partly support the usefulness of the variables in explaining intended condom use with a casual/new partner. Self-efficacy, some beliefs about consequences of using comdoms, perceptions of threat, optimistic infection risk and internal Health Locus of Control are related to intention to use condoms. Their predictive power, however, is rather small.

Social influence, a determinant of behavioural changes among homosexuals (McCusker, Zapka, Stoddard \& Mayer, 1989) was not included in this study. From this perspective it is a major weakness of this investigation, because an unknown amount of variance in the prediction of intention to use condoms is relatively uninterpretable in the context of the Theory of Reasoned Action. Yet, a study among Dutch adolesents showed that social norms predicted (after attitude and self-efficacy) only a small proportion of the variance in intended condom use (Schaalma, Kok \& Peters, 1993), while social norms were not found to be a predictor of condom use in another study among adolescents (Richard, ot al., 1991).

To summarise, the results reported here showed that it is not enough to convince heterosexuals that they should change their high risk sexual behaviour. They atso need guidance in how to translate their concerns into beliefs that are consistent with condom use. Additionally, persons who believe they are susceptible to HIV infection and therefore seek an HIV test, form a self-selected sample with regard to face-to-face interventions. Especially posttest counselling should therefore be revised to include training in communication skills necessary to negotiate in sexual relationships. Finally, more research is necessary to determine which factors influence a person's self-efficacy expectations. 


\section{DETERMINANTS OF CONDOM USE AMONG ADOLESCENTS}

Published as: Leenaars, P.E.M., Rombouts, R., Lammerdin, A.G., Zwetsloot, L.M. (1992). Determinanten van condoomgebruik onder jongeren. Gedrag en Gezondheid, 19(2), 77-87.

\subsection{Introduction}

Sexually Transmitted Diseases (STDs) are generally described as infectious diseases, which are mainly (exclusively) transmitted through sexual intercourse (WHO, 1986).

Many changes have occurred in curative and non-curative STD health care in the last few years. Until 1980, attention was primarily focused on classic STDs (such as gonorrhea and syphilis). After 1980, more emphasis was put on the problems around viral STDs (such as Chlamydia trachomatis, Herpes Simplex Virus, Human Papilloma Virus, and the Human Immune Deficiency Virus) the "second-generation STDs", which surpass the classic STDs in incidence and gravity of complications (arising infections, infertility, ectopic pregnancy). Not only the complications of untreated and untreatable STDs, but also the increased risk of cervical cancer with the presence of ulcerous STDs, indicate the importance of both primary prevention and early detection of STDs. Besides STDs with symptoms, especially complications of STDs that pass without symptoms or are coupled with vague symptoms, lead to the necessity of quick and adequate treatment. Precise information about the incidence of STDs is not available. It can be stated that STDs are not randomly divided among the population. The highest incidence is found in the younger age brackets: the group aged 20 to 24, followed by the group aged 15 to 19 and persons aged 25 to 29 (Meyer, 1988). It is estimated from the registration data of positively diagnosed gonorrhea- and syphilis cases that 20,000 adolescents aged 15 to 25 contract an STD in the Netherlands each year (STD foundation, 1988). Adolescents aged 15 to 24 , therefore, form an important target group for education about STD prevention.

Research into the sexual activities of adolescents in urban areas spread over the Netherlands, shows that in most cases about one-third of the students aged 14 to 17 has experience with sexual intercourse. A research project of Gieling and Heuzer (1988) among 500 students from Amsterdam showed that $3 \%$ of the adolescents aged 12 to $14,22 \%$ of those aged 14 to 15 and $52 \%$ of those aged 16 to 17 have sexual relationships. Furthermore, Delil (1988) established from data of 505 students in the eastern part of Zuid-Limburg, aged 14 to 16 , that $33 \%$ of these adolescents have experience with sexual intercourse. De Wit, Kok, Timmermans and Wijnsma (1990) also achieved similar results. Whether these findings also apply to rural areas is as yet unknown. In this age of 
puberty, exploring sexuality often leads to several sexual relationships and consequently to an increased risk of contracting an STD.

Condom use is considered to be one of the most effective methods of preventing venereal diseases. However, it appears from a number of studies into condom use among sexually active students, that the majority of adolescents Irregularly or never use them (De Wit et al. 1990, Delil, 1988, Gieling \& Heuzer, 1988). Research into determinants of condom use shows that contraception, knowledge about the effectiveness of condom use, the belief that condom use is a sign of distrust (Sandfort, De Vroome, Paalman \& Tielman, 1989), social acceptance of condom use, knowledge about the prevention of STDs and the extent to which condom use is experienced as pleasant (Luykx, Marsman \& Van der Rijt, 1989) play a part in condom usage. Moreover, Wijnsma (1990) adds several factors to this, based on poster and paper presentations, namely: risk estimations, ability to talk about sex, use of alcohol and drugs, perceptions of beliefs of peers, access to condoms, beliefs about buying condoms, beliefs about the influence of condoms on sexual feelings and sexual encounters.

In order to encourage condom use, it is first of all necessary to establish which factors impede and stimulate condom use. Azjen and Fishbein (1980) state that the intention to perform health behaviour depends chiefly on two factors: a person's attitude (a combination of beliefs about expected behavioural consequences and the evaluation of these consequences) and the subjective norm (a combination of perceived beliefs of others to demonstrate the behaviour as well as the motivation to comply to these beliefs). According to this theory, the intention of performing health behaviour is the most important predictor of the reccommended behaviour and a change only takes place when a change in beliefs has occurred. Whether a change in beliefs also results in a change of behaviour depends, according to Stretcher, De Vellis, Becker and Rosenstock (1986) on the assessment people make with regard to the expected effectiveness of the behaviour in question and self-efficacy expectations. Bandura (1986) also achieved similar findings.

In this article, we investigated which secondary school children use condoms and which determinants explain the intentions of using condoms during sexual intercourse with casual partners. Several variables from Azjen and Fishbein's model were used, in particular: beljefs about condom use, perceived beliefs of athers about condom use, evaluation of condom use and the intentions of using condoms with casual partners. Moreover, several other variables were added to the model: previous condom use, the expected effectiveness of condom use and the estimated risk. 


\subsection{Methods}

\section{Procedure and sample}

In order to answer these questions a questionnaire was distributed among secondary school children in the Hague. From February to March of 1988, two schools of technical and vocational training for 16-18-year-olds, three schools for part-time education and the highest grades of four comprehensive schools participated in this study. These schools were selected with regard to a wide distribution of the types of schools. A structured questionnaire was completed in class and collected afterwards in a sealed envelope.

The sample consisted of 130 men (48\%) and 140 women (52\%). The mean age of the respondents was 18 , of which $15 \%$ fall in the 20 to 22 years age bracket. The division by school type is as follows: $14 \%$ part-time education, $24 \%$ technical and vocational schools, 31\% secondary modern school and $31 \%$ grammar school. More than half of the students are of Dutch descent $(69 \%)$ and one-fifth are of Surinam descent $(21 \%)$. The remaining participants are of Turkish, Moroccan, Moluccan or Antillean descent (10\%). Half of the students $(51 \%)$ indicate to be non-religious and the remaining participants are RomanCatholic $(13 \%)$, Protestant-Christian (15\%) or Islamic, Hindy (21\%).

\section{Measures}

The following variables were used in this study:

- Social-demographic and individual characteristics, such as gender, age, education, cultural background, sexual activities, sexual preference and number of sexual partners;

- Beliefs about condom use. Beliefs about aspects of condom use have been measured with a questionnaire consisting of eleven items that were partially adopted from Luykx, Marsman and Van der Rijt (1989). The respondent indicated on a 5-point scale to what extent they agree with a statement about condom use $(1=$ disagree entirely to $5=$ agree entirely). Factor analysis: (oblique) on these items has yielded three factors (explained variance through these three factors is $53 \%$ ) each with an acceptable level of internal consistency, namely:

- beliefs about planning condom use (four items, Cronbach's alpha $=0.59$ ) with as the most important item: "those who always have condoms on them, are looking for sex":

- beliefs about buying condoms and the cost of them (three items, Cronbach's alpha $=0.70$ ) with as the most important item: "I find it difficult to buy condoms from a dispenser";

- beliefs about condom use as a disruptive factor (four items, Cronbach's alpha $=0.69$ ), with as the most important item: "putting on a condom while having sex is an annoying interruption". 


\section{STD PREVENTION}

There is a poor relationship between the factors beliefs about planning condom use and costs of condom use $(r=0.31 ; p<.001)$, while there is no relationship between the factors: beliefs about planning condom use and beliefs about condoms as having a disruptive effect $(r=0.14)$ and between the factors: costs of condom use and the disruptive effect of condom use ( $r=$ 0.14 ). With reference to the analogy between the factors in relation to each other and other variables included in the study, the scores of the factors in the tables are transformed to a 5-point scale;

- Perceived beliefs of significant others: (three items, Cronbach's alpha=0.60) 'I suspect that my mother (father, friends) think that I should use condorns': ( $1=$ disagree entirely to $5=$ agree entirely):

- Singular questions on:

- beliefs about the expected effectiveness of condom use: "I can avoid a venereal disease by suggesting to my partner that we use condoms, and making sure that we do";

- beliefs about the combination pill and condom use: "when every woman is on the pill, condoms are unnecessary";

- beliefs about condom use with several partners: "when you have sex with multiple partners, it is wise to use condoms";

. beliefs about condom use in the presence of an STD. "I will only have sex without using a condom when I am absolutely sure that neither I nor my partner have a VD";

- estimated infection risk. "how high do you estimate the risk of you contracting a venereal disease?" ( $1=$ no risk to $5=$ very high);

- evaluation of condom use: "how important/bothersome is it to you when your partner suggests to use condoms and making sure that you do?' (1= completely unimportant/not bothersome at all to $5=$ very important/ very bothersome):

- frequency of previous condom use with a casual partiner. $(1=$ never to $4=$ always):

intention of using condoms with a casual partner ( $1=$ never to $5=$ always).

\section{Data analysis}

Statistical analyses were conducted with the statistical software program SPSSpc 3.1 (Norusus, 1987).

The central analysis pertains to the question which combination of beliefs about condom use can explain the intention of using condoms. For that purpose, multiple-regression analyses were used. At the same time the effect of socialdemographic charateristics on beliefs about condom use and the previous use of condoms was studied by means of multivariate analyses. Chi-squared analysis have been used to retrieve relationships between social demographic characteristics and sexual activity. 


\subsection{Results}

Soclal-demographlc characterlstics of the sexual active

A total of 144 adolescents $(54 \%)$ who participated in this sample were sexually active in the past year. About $67 \%$ of these had one and $33 \%$ had two or more sexual partners. These students have a mean age of 18 years. More than half are men $(58 \%)$ and the majority attend technical and vocational schools $(29 \%)$ or lower general secondary schools, secondary modern school $(33 \%), 18 \%$ attend school part-time, and $19 \%$ attend grammar school. Compared with adolescents who have not had sexual relations yet, the sexual active ones are relatively more often men $\left(\chi^{2}=88.9 ; d f=1 ; p<.001\right)$ and they attend grammar school relatively less often $\left(\chi^{2}=18.9 ; d f=3 ; p<.001\right)$. Moreover, these students are usually slightly older (mean age $=18$ ) than those who have no sexual experience (mean age $=17)(F=13.7 ; p<001)$.

\section{Previous condom use}

Almost one-third of the sexually active respondents (30\%) presently never use condoms, $45 \%$ use them on an irregular basis and $25 \%$ indicate to always use condoms. The social-demographic characteristics gender, age, education, cultural heritage and religion do not show a relationship with the frequency of previous condom use. Just the same, no relationship has been established between the type of relationship and actual condom use. Those who have had sexual intercourse with one single partner in the past year (monogamous relationship) use condoms as often as those who have had intercourse with several partners (serial monogamy or polygamy).

Slightly more than half of the sexually active pupils $(54 \%)$ consider the risk of contracting an STD low to impossible. Moreover, one-fifth of the sexually active pupils $(21 \%)$ are still of the opinion that condom use is unnecessary if all women are on the pill. Multivariate analysis was conducted to examine whether the sexually active students who never, irregularly or always use condoms differ in beliefs about condom use. This analysis showed that the sexually active students who never, irregularly or always use condoms, differ in a combination of beliets about condom use (Hottelings' $T^{2}=.50 ; p<.001$ ). The results of the univariate tests indicate which beliefs are responsible for the established difference between sexually active students who never, irregularly or always use condoms.

Table 7 shows that the respondents who always use condoms, consider condom use more important and less bothersome and consider them more effective in preventing venereal diseases than those who never or irregularly use condoms. Likewise, these adolescents have more positive beliefs about the planning, the purchase and the costs of condom use, as opposed to those who never or irregularly use them. On the other hand, persons who irregularly use 
condoms have a more positive belief about their disruptive effect than those who never or always use them. Moreover, it is shown that persons who never, irregularly or always use condoms do not differ in the estimated risk of contracting an STD and the belief that being on the pill renders condom use unnecessary.

TABLE 7. MEAN SCORE AND F-VALUES ON BELIEFS ABOUT CONDOM USE BY ACTUAL CONDOM USE.

\begin{tabular}{|c|c|c|c|c|c|}
\hline \multirow{2}{*}{ 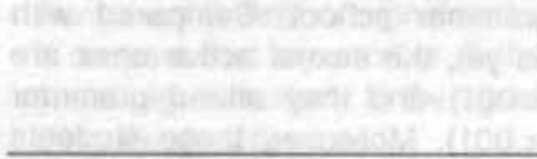 } & \multicolumn{3}{|c|}{ PAEVOUS CONDON USE } & \multirow[b]{2}{*}{$\begin{array}{c}\text { F- } \\
\text { value }\end{array}$} & \multirow[b]{2}{*}{$\begin{array}{l}\text { elgn. } \\
\text { Lovol }\end{array}$} \\
\hline & nover & Irregular & alwaye & & \\
\hline Estimated Infection riak & 2.5 & 2.6 & 2.4 & 0.9 & .400 \\
\hline Importance of condom use & 2.8 & 3.6 & 4.3 & 16.6 & .000 \\
\hline Annoyance of condom use & 3.1 & 3.0 & 2.2 & 5.7 & .004 \\
\hline Percelved offectiveness & 3.2 & 3.7 & 4.2 & 10.1 & .000 \\
\hline Planning condorn use* & 3.3 & 3.6 & 3.9 & 4.5 & .010 \\
\hline Pureheseo/cosis of condoms" & 3.0 & 3.0 & 4.1 & 2.0 & .050 \\
\hline Disruptive effect of condoms* & 2.0 & 3.1 & 2.5 & 3.6 & .030 \\
\hline $\begin{array}{l}\text { Boing on the pill renders condom use } \\
\text { unnecessary }\end{array}$ & 2.1 & 1.8 & 2.0 & 0.9 & .390 \\
\hline
\end{tabular}

N.B. score range: 1 = low agreement;

$$
1 \text { = entirely agree; } \quad 5 \text { = low agreement }
$$

The Intention of using condoms with a casual partner

Almost two-third of sexually active students intend to always use condoms with casual or different partners, while the remaining sexually active students intend to irregularly $(30 \%)$ or never $(6 \%)$ use condoms with a casual partner. Gender is related to the intention of using condoms with a casual partner $\left(\chi^{2}=3.7 ; d f=1 ; p<\right.$.05). Slightly more than half of the sexually active males $(54 \%)$ intend to always use condoms with a casual partner, while this percentage is clearly higher for the sexually active females $(74 \%)$.

The demographic characteristics age, education, religion and cultural background do not show a relationship with the intention of using condoms with casual partners.

To find out which beliefs about condom use (from Table 7) in addition to previous condom use now play a part in the intention to use condoms with casual partners, multiple-regression analysis was conducted for the total group of sexually active students. Apart from previous condom use only beliefs about the expected effectiveness of condom use contributes to the explanation of the intention to use them with casual partners. The explained variance of the 
intention to use condoms with a casual partner amounts to $26 \%$. However, when we add the variable 'perceived necessity of condom use when having sex with different partners', beliefs about condom use with an STD and the perceived beliefs from mothers, fathers and friends (peers) to this analogy, the explained variance can consequently be increased to $41 \%$. The variables that play a part in the explanation of the intention to use condoms with casual partners are shown in Table 8.

TABLE 8. RELATIONSHIPS BETWEEN BELIEFS ABOUT CONDON USE, PREVOUS CONDOM USE AND INTENTION TO USE CONDOMS WITH A CASUAL PARTNER

\begin{tabular}{lrrr}
\hline PAEDICTORS & r & partial r & MR \\
\hline previous condom use & & & 0.45 \\
Condom use when having sex with different partners & 0.33 & 0.31 & 0.45 \\
Perceived effectiviness of condom use & 0.45 & 0.21 & 0.54 \\
Condom use with an STD & 0.37 & 0.22 & 0.60 \\
Perceived beliefs of others & -0.08 & -0.21 & 0.64 \\
\hline
\end{tabular}

noto: only significant predictors $(p<.05)$ are shown

explained variance $=41 \%$

Table 8 shows that the intention of using condoms with casual partners is related to previous condom use, beliefs about the necessity of condom use when having sex with multiple partners, the expected effectiveness of condom use, beliets about the necessity of condom use with an STD and the perceived beliefs of significant others about using them. On the other hand, the variable beliefs about the costs, bias about planning and beliefs about the disruptive effect of condom use do not contribute to the prediction of intended condom use with a casual partner. The explained variance has now decreased to $38 \%$ and the belief about the expected effectiveness of condom use is in this comparison the most important determinant of intended condom use. In order to find out whether the difference in intention to use condoms among sexually active males and sexually active females can be explained because males and females differ in beliefs about condom use, another stepwise multiple-regression analysis was conducted for both groups once more (see Tables 9 and 10).

TABLE 9. RELATIONSHIPS BETWEEN BELIEFS ABOUT CONDOM USE, PREVOUS CONDOM USE AND THE INTENTION TO USE CONDOUS WHEN HAVING SEX WITH A CASUAL PARTNER FOR FEMALES ( $N=61$ )

\begin{tabular}{lcccc}
\hline PREDHCTORS & I & partial r & MR \\
\hline Porceived importance of condom use & 0.48 & 0.48 & 0.48 \\
\hline
\end{tabular}

explained variance $23 \%$ 
Table 9 shows that the intention among females to use condoms with a casual partner can only be explained by the experienced importance of using condoms. The remaining variables in the analysis do not contribute significantly to the explanation of the intention of females to use condoms with a casual partner.

TAELE 10. RELATIONSHIPS BETWEEN BELIEFS ABOUT CONDCM USE, PAEVOUS CONDOM USE AND THE, INTENTION TO USE CONDOMS WHEN HAVING SER WITH A CASUAL PARTNER FOR MALES $(\mathrm{N}=83$ )

\begin{tabular}{lccc}
\hline PREDICTORS & r & partial r & MR \\
\hline Eflectivenoses of condom use & 0.48 & 0.53 & 0.48 \\
Condom use with multiple partners & 0.48 & 0.52 & 0.60 \\
Condom use with an STD & 0.47 & 0.43 & 0.67 \\
Beliefs of significant others & -0.19 & -0.37 & 0.70 \\
Previous condom use & 0.43 & 0.33 & 0.74 \\
Disuptive offect of condorn & 0.24 & 0.30 & 0.75 \\
\hline
\end{tabular}

note: only significant predictors $(p<0.05)$ are shown explained varience $=57 \%$

Table 10 shows that intended condom use with a casual partner among males is related to the expected effectiveness of condom use, beliefs about the necessity of condom use when having sex with multiple partners, beliets about condom use with an STD, perceived beliefs of significant others about condom use. The variables that do not contribute significantly in inteded condom use are: beliefs about costs of condom use and bias about the planning condom use.

\subsection{Discussion}

Just over half of the 270 students who have participated in this study had sexual intercourse during the past year. However, the majority of these sexually active pupils are presently never or irregularly using condoms. These results are virtually in line with the findings of Gieling and Heuzer (1908) and De Wit et al. (1990). Whether these findings about sexual behaviour among adolescents in urban areas can be generalised to secondary school pupils in rural areas remains to be seen. Moreover, we should ask ourselves to what extent the selfreport on sexual behaviour raises socially desirable answers among adolescents. However, the influence of this possible over or underreporting of sexual behaviour among adolescents is unestimable.

Unprotected intercourse with casual partners increases the risk of contracting an STD. Insights into the determinants of condom use during sexual intercourse with casual partners is, therefore, important in decreasing the relatively high 
incidence of STDs among adolescents. It can be stated that these students estimate the risk of an STD for themselves to be very low, while this risk of an STD, viewed objectively, is definitely present. This low estimated risk may have to do with the phenomenon "unrealistic or optimistic bias", the tendency of estimating the risk of getting a disease lower for yourself than others (Weinstein, 1983). Cognitive errors (for example bias) that, according to Weinstein (1983), apply to the explanation of unrealistic bias, may play a part in the lowly estimated risk of contracting an STD. This means that, when educating sexually active adolescents, extra attention should be paid to establish misconceptions or bias about contracting an STD (for example, an STD only occurs among homosexual men). In this respect, it is therefore advisable to stress the relatively high incidence of STDs particularly among adolescents, the high-risk sexual behaviours as well as the asymptomatic development of a number of STDs among women.

It is repeatedly stated in the literature that condom use among adolescents can chiefly be explained by the use of condoms as contraceptives (Sandfort, Vroome, Paalman \& Tielman, 1989). However, it appears from our study that there is no discrepancy between those who never, irregularly or always use condoms in the belief that "when on the pill, condom use is unnecessary". Contrary to the findings of Luykx, Marsman and Van der Rijt (1989) $(40 \%$ of 628 respondents) $21 \%$ of the 144 sexually active pupils more or less agrees with this statement. This means that the majority of these sexually active students are of the opinion that condom use (in addition to a contraceptive) can also serve another purpose. The possibility exists that the discrepancy in the findings are caused by the difference in moments of measurement: before or after national educational campaigns about condom use.

That the type of relationship plays a part in previous condom use is not confirmed in this study. Those who had sexual intercourse with one single partner (monogamous relationship) during the past year use condoms as often as those who had sexual intercourse with multiple partners (serial monogamy or polyga$\mathrm{my}$ ). Beliefs about the desired behaviour do play a role in previous condom use of these sexually active students. Adolescents who always use condoms, consider condom use more important, less bothersome and more effective in the prevention of venereal diseases, as opposed to those who never or irregularly use them. Furthermore, pupils who always use condoms have more positive beliets about planning and buying them and the costs of condom use. These findings results in suggestions for educational purposes; adolescents who never or irregularly use condoms, need to be convinced of the importance of using condoms and of the effectiveness of condom use concerning STDprevention. Furthermore, it is advisable for the educator to go imto the bias against planning condom use, such as: emphasising that condom use is socially accepted and that condom use can be seen as a sign of responsible behaviour. 
Moreover, it is advisable to establish what exactly is considered to be bothersome about buying condoms in shops and from dispensers. The taboo on sexuality and STDs probably plays a part in this. In that case, it is also desirable for the educator to make the taboo of STDs a subject of discussion and give adequate instructions (if necessary followed by an exercise) about the planning of condom use and purchasing condoms. Finally, certain beliefs about the disruptive effect of condoms deserve particular attention. The educator can prepare the pupils who never use condoms about unexpected negative symptoms that can occur (such as: putting on a condom for the first time takes time and this could affect arousal and erection). Secondly, it should be stressed that the effect of condom use as a preventative device for STDs is expressed in the knowledge that you have been optimally protected. In earlier research among secondary children (Leenaars, Rombouts, Lammerdin \& Zwetsloot, 1990) we found it worthwile to take the sexual experiences of those involved in consideration and to encourage prevention of STDs by making STDs a subject of discussion in sexual relationships. In order to reduce the anticipated disruptive effect of condoms it is advisable to give detailed instructions about negotiating the prevention of STDs through condom use and, if necessary, followed by exercises about actual condom use.

The analyses of the intention to use condoms show that despite all the information only slightly more than half of these sexually active adolescents intend to always use condoms during sexual intercourse with a casual partner. Moreover, we have established that previous condom use is the most important predictor of the intention to use condoms in the future. Also, beliefs about the expected effectiveness of condom use, perceived necessity of condom use with multiple partners, condom use with an STD and the perceived importance of condom use contribute to the explanation of intended condom use. This means that once more factors which deal with previous condom use, form pretexts in STD prevention education. Furthermore, it is advisable for educational purposes, to pay extra attention to beliefs about condom use, such as: the importance of using condoms when having sex with multiple partners, the effectiveness of using condoms as a preventative device for STDs and the importance of using condoms with an STD. That the perceived beliels of others (father, mother, friends) about condom use have a negative influence on the intention of going to use condoms, can probably be explained through the age bracket (puberty) in which these edolescents find themselves and because the sexual activities of these secondary students take place despite the possibly restrictive beliefs of their parents.

It is remarkable that bias about planning condom use, purchase and costs of condoms and the disruptive effect of condoms do not play a role in intended condom use, while these factors are indeed related to previous condom use. In 
this case, the annoying aspects of condom use probably have an especially negative influence on the desired behaviour in the short term (habits).

The fact that females intend to use condoms more often than males stems from the difference in beliefs about condom use. Intentions of using condoms among males can chiefly be explained by beliefs about the expected effectiveness of condoms, previous condom use with multiple partners, condom use with an STD, and the disruptive effect of condoms. On the other hand, the intention of using condoms among females can only be explained by the perceived importance of using them. However, the explained variance is very low. This means that factors other than the ones researched here play an important part in the females' intention of using condoms with a casual partner. When educating high school students, a difference in beliefs about condom use between females and males should be taken into account.

In the Netherlands, an increasing amount of attention is paid to the development of educational material for adolescents about Sexually Transmitted Diseases and their prevention. The above research results and recommendations implicate that particularly the experience with condom use and specific beliefs about condom use are important themes in encouraging future condom use with casual partners. Moreover, there are indications that, especially when educating females about condom use, other themes should be covered as well. Finally, it is advisable to find out in a second study into condom use, which factors play a part in the females' intention to use condoms with a casual partner. 


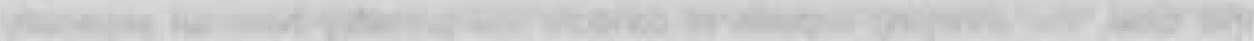

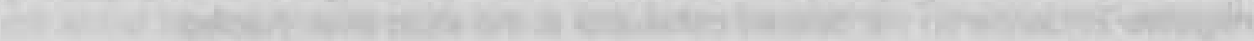

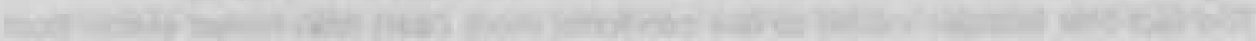

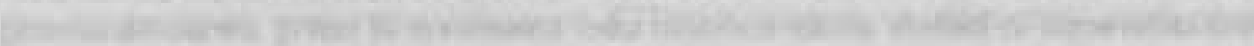

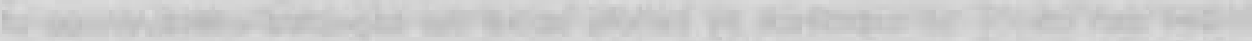

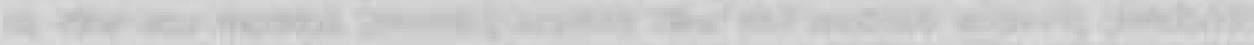

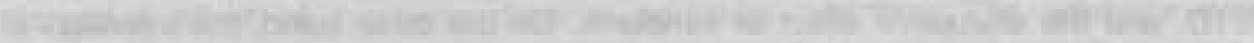
wit

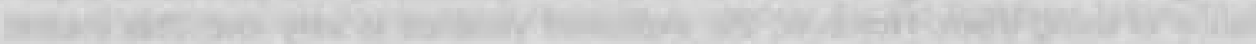

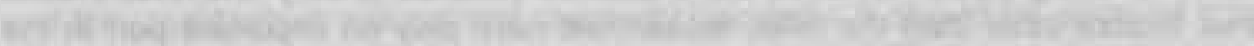

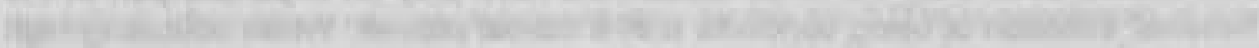

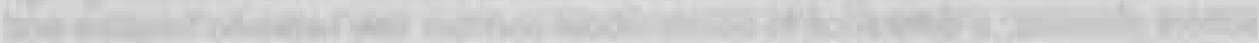

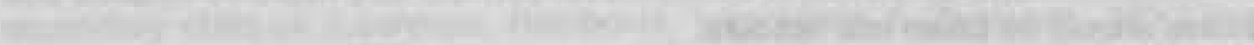

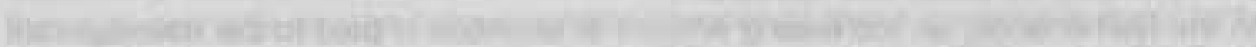

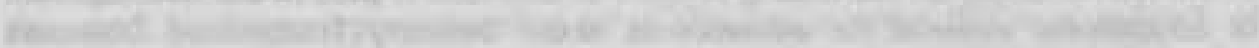

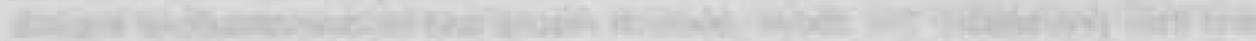

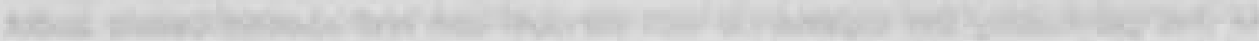

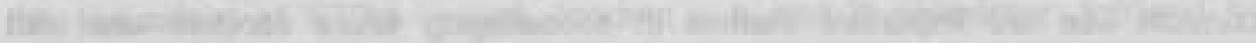

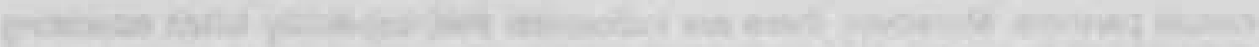

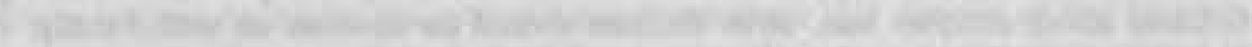

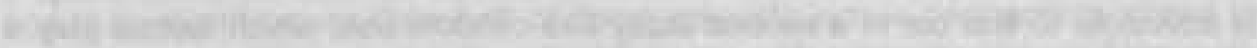

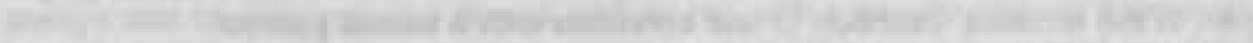

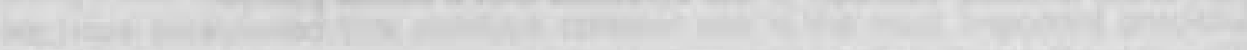

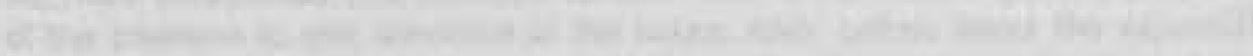

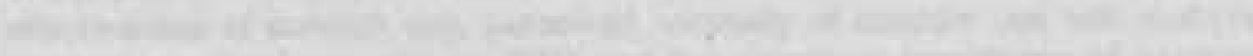

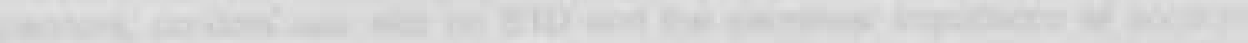

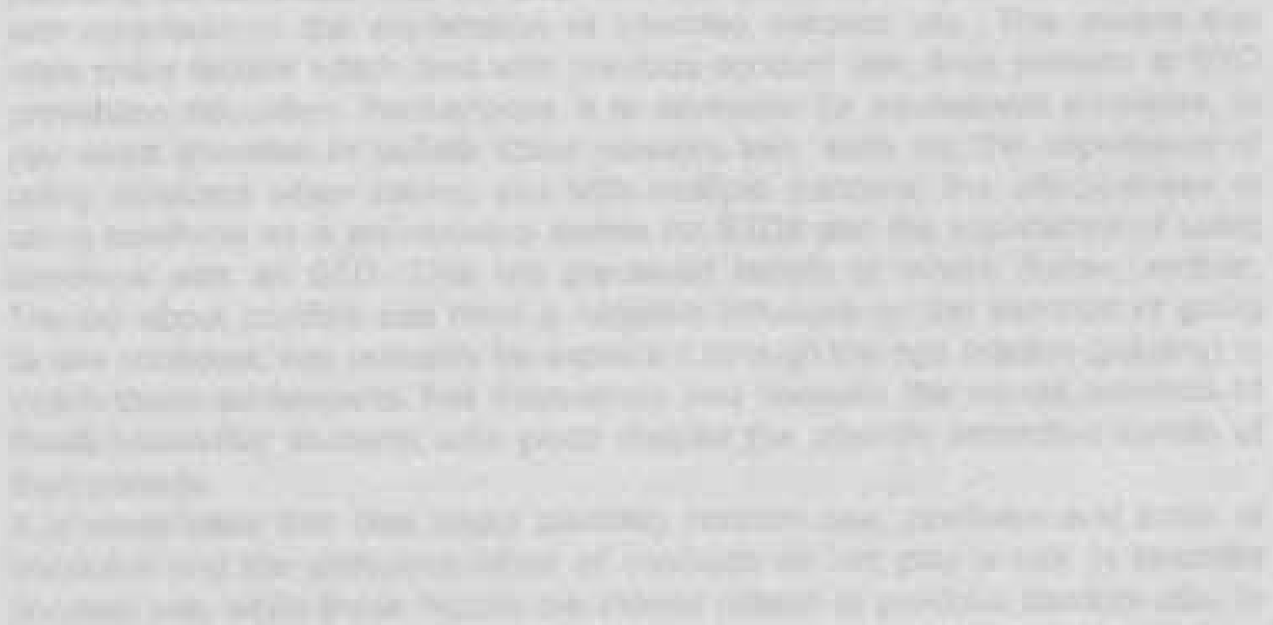




\section{BELIEFS, EVALUATIONS AND PREVENTION OF SEXUALLY TRANSMIT- TED DISEASE AMONG SECONDARY SCHOOL PUPILS}

Published as: Leenaars, P.E.M., Rombouts, R., Lammerdin, A.G., Zwetsloot, L.M. (1990). Beliefs, evaluaties on preventie van Sekueel Overdraagbare Aandoeningen onder middelbare scholieren. Tijdschrift voor Gezondheidsbevordering, 11, 2-12.

\subsection{Introduction}

The interest in Sexually Transmitted Diseases (STDs) in general, and in regarding AIDS in particular, has increased dramatically in the past year. Especially the interest in the secondary transmission of the HIV virus ftransmission of HIV by traditional risk groups, such as: homosexual and bisexual men, prostitutes and intravenous drug users, to the general public) has received much attention in the daily papers and scientific literature. It is suspected that the secondary transmission of HIV is mainly transmitted by the group of intravenous drug users. Apart from this, research showed that the risk of HIV infection is strongly associated with infections of STDs which are accompanied by genital warts (Quinn, 1988; Holmberg. Stewart \& Russell, 1987). Not only the risk of HIV infection but also the long term complications of untreated STDs increase the necessity for primary prevention of STDs. Complications in this context are Pelvic Inflammatory Diseases (PID) and their late sequelae such as infertility and ectopic pregnancy.

In several foreign studies (McCormach, Rossner \& McComb, 1985) a high incidence of Chlamydia infections was found among adolescents. Due to a lack of reported cases of a Chlamydia infection the prevalence of this STD is unknown in the Netherlands. Based on the reported cases of STD clinics it is estimated that 20,000 adolescents aged 15 to 25 acquire an STD in the Netherlands annua!ly (SOA-stichting, 1988).

The high incidence of STDs emong adolescents is probably related to the lack of adequate skills to prevent them. Adolescents start new sexual relationships which is often accompanied by frequent partner-changing. It is usually a matter of impulsive behaviour; nothing is planned beforehand. Aiso biases to STDs and unfamiliarity with safe-sex practices may contribute to the high incidence of STDs among adolescents. Until recently, education about STD prevention focused mainly on increasing knowledge. However, knowledge about the source of the disease is an insufficient basis to encourage health behaviour (Fishbein \& Ajzen, 1975). Azjen and Fishbein (1980) assume that the intention to demonstrate health behaviour, depends in particular on two factors: a person's attitude (combination of expected consequences of the behaviour and the evaluation of 
these consequences) and the subjective norm (combination of the perception of other important person's opinions and the degree to which an individual is inclined to conform to these opinions). According to this model a change in behaviour only occurs when one can speak of a change in beliefs.

According to Stretcher, De Vellis, Brecker and Rosenstock (1986), it partly depends on the way these people assess the anticipated effectiviness of the behaviour and personal effectiveness, whether a change in beliefs also brings about a change in behaviour. Bandura (1986) also stated that behaviour and behavioural change depends on two components (1) the assessment that the demonstrated behaviour will lead to the desired result (behaviour effectiveness) and (2) assessing one's own abilities to be able to carry out the behaviour successfully (personal effectiveness). In a recent study, Paulussen, Kok, Kinibbe and Cramer (1990) integrated both theories (of Fishbein \& Azjen, and Bandura) and Investigated these determinants of HIV-preventive behaviour among intravenous drug users. Paulussen et al. (1990) assumed that behaviour effectiveness can be considered a part of the attitude and that the personal effectiveness next to the attitude and social norm contribute significantly to the prediction of health behaviour.

In this article we describe the relationships between knowledge, beliefs about effectiveness of STD-preventive behaviours, evaluation of STD-preventive behaviour and the previous STD-preventive behaviour on the one hand and the intention to STD-preventive behaviour among secondary pupils on the other. The highest risk behaviour for acquiring an STD is chiefly unprotected sexual intercourse with frequent partner-changing, and unprotected sexual intercourse with anonymous sexual partners. We assumed that STD prevention consists of several partly complimentary behaviour alternatives or skills.

\subsection{Mothods}

\section{Procedure and sample}

In February and March of 1988 a written questionnaire was administered to a random sample of secondary school pupils in the Hague. Eleven secondary comprehensive schools were selected per school type. Eventually; two technical and vocational schools, three schools for part-time education and the highest grades of four comprehensive schools particpated in this study. In order to have an equal distribution of the various grades, we visited several classes in these schools.

The questionnaires were filled in written and anonymously in class supervised by the research staff and were collected afterwards in a sealed envelope. Two out of the 308 pupils refused to participate. Furthermore, 36 questionnaires with more than 7 answers missing were removed. Because of this selection procedu- 
re 270 useable surveys eventually remained. The random check consisted of 130 men (48\%) and 140 women (52\%). The mean age of the respondents was 18 years, of which $15 \%$ falls in the 15 - to 16 -year-old age group, $71 \%$ in the 17 to 19-year-old age group and $14 \%$ in the group of $20-22$ year olds. The selection per school type was as follows: $14 \%$ part-time education, $24 \%$ technical and vocational education, $31 \%$ secondary modern school, and $31 \%$ grammar school. More than half of the pupils were of Dutch descent $(69 \%)$ and one-fifth was of Surinam descent (22\%). The remaining students were of Turkish, Moroccan, Moluccian or Antilian descent. Half of the students (51\%) indicated that they were non-religious and the remaining participants were Roman-Catholic (13\%), Protestant-Christian (15\%) or Islamic/Hindy (21\%).

\section{Measures}

The following variables were measured in this study:

- Social demographic and individual characteristics, such as: gender, age, school attendence, origin, living arrangements, sexual activities, sexual preference and number of sexual partners.

- Knowledge about STDs was determined by means of a knowledge questionnaire of Luykx, Marsman and Van der Riijt (1987). This questionnaire consisted of 22 items with three response catagories (correct-incorrect-don't know). Three new variables were calculated: correct knowledge, incorrect knowledge and unknown knowledge. The internal consistency of the questionnaire was calculated by Cronbach's alpha $=0.56$. The correct knowledge score was obtained by summation of the number of correct answers (maximum score $=22$ ).

- Expected effectiveness of STD-preventive behaviours was measured by means of a self-designed questionnaire, consisting of 13 items. The respondent indicated on a 5-point scale for each behaviour alternative to what extent he agreed with the items, such as: "I can prevent acquiring a venereal disease by always using a condom"; Cronbach's alpha $=0.72$. The maximum score attainable was 65 ; respondents are strongly convinced that all actions lead to prevention of STDs.

- Evaluation of STD-preventive behaviour is determined by assessing 13 items about STD-preventive behaviour alternatives on a 5-point scale in terms of perceived importance and perceived annoyance. Examples of the items are: "Asking my partner whether he or she has a vd, is ..... and "always using condoms with a casual partner, is ....." ; 13 items on the importance scale; Cronbach's alpha $=0.82$ and 13 iterns on the annoyance scale, Cronbach's alpha $=0.78$. The maximum sumscore attainable for both variables was 75 , indicating that respondents assess the various behaviour alternatives as very important to very annoying respectively. 
- Previous STD-preventive behaviour has been determined by indicating on a 4-point scale to what extent respondents are using different STD-preventive actions; 13 items, Cronbach's alpha $=0.77$. The maximum sumscore attainable indicates that the past sexual behaviour is strongly focused on prevention of contracting an STD.

- Intention to STD-preventive behaviour has been determined by indicating on a 4-point scale how often the respondents think they will use the twelve STD-preventive actions in the future. The internal consistency amounts to Cronbach's alpha $=0.82$. The maximum sumscore attainable was 48 ; respondents are strongly inclined to focus their sexual behaviour on preventing an STD in the future.

\section{Data analyses}

Statistical analyses were conducted with the statistical software package SPSSPC 3.1 (Norusis, 1988). Univariate analyses of variance were conducted to study the effects of social demographic characteristics on independent variables. Next, stepwise multiple regression analysis was used to answer the central question. The central question is how to retrace variation in criterium variable (here: intention to STD preventive behaviours) on a variation in the combination of determinants (here: behaviour effectiveness, importance and annoyance of STD preventive behaviour, previous STD-preventive behaviour and knowledge).

\subsection{Results}

Soclal demographlc and Indlvidual chracteristlcs

As stated earlier the study population consisted of 130 men (48\%) and 140 women $(52 \%)$ with a mean age of 18 years. Of all respondents more than twothirds live with their parents $(69 \%)$, almost one-quarter belongs to a singleparent family (21\%) and the others (remaining respondents) live on their own or live together with a partner $(10 \%)$. The majority of the respondents $(96 \%)$ have a heterosexual preference.

In this study, those who have had sexual intercourse and where contact has been made between genitals or mouth-anal genitals belong to the sexually active. Of the 270 respondents more than half $(54 \%)$ have been sexually active in the past year, of which $35 \%$ had one sexual partner, $6 \%$ had two partners and $13 \%$ had more than two partners. Of the sexually active almost more than half had sexual intercourse one to three times a month $(44 \%)$ and $53 \%$ had serual intercourse one to several times a week. Of the total study population $6 \%$ have been examined for venereal disease once or more than once.

A relationship exists between gender and nurnber of sexual partners $\left(\chi^{2}=15.5\right.$; $d f=2 ; p<.001$ ). Well over one-third of the men had no sexual partner in the past 
year (36\%), $40 \%$ had one partner and one-quarter had more than one partner (25\%), while these percentages for women were $56 \%, 33 \%$ and $11 \%$ respectively. A relationship also exists between type of school attended and number of sexual partners $\left(\chi^{2}=22.8\right.$; $\left.d f=6 ; p<.001\right)$. Figure 5 shows the division of the number of sexual partners of the respondents by type of school.

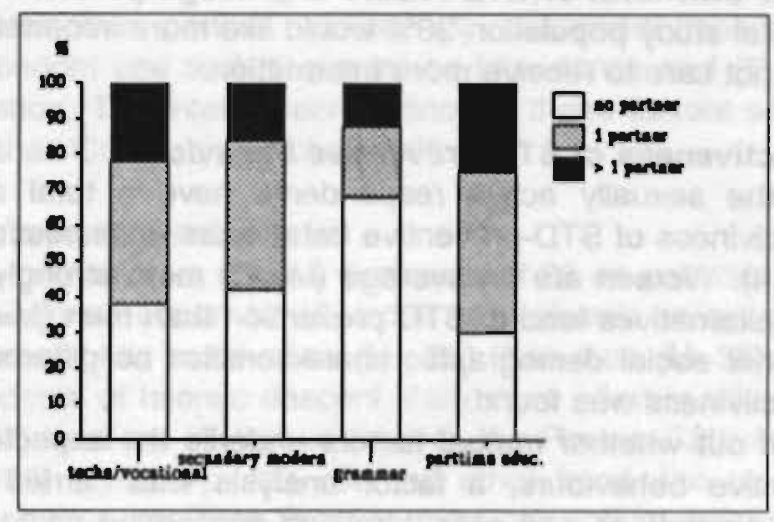

FIGURE 5. NUMBER OF SEXUAL PARTNERS BY TYPE OF SCHOOL

Respondents who attend grammar school indicate more often that they did not have a sexual partner in the past year and those who attend secondary modern school, technical and vocational schools and part-time education are more likely to have one partner. Furthermore, respondents who attend school part-time and who attend technical and vocational schools are more likely to have had more than one sexual partner in the past year than those of secondary modern schools and grammar schools. The religion of those involved was related to the number of sexual partners $\left(x^{2}=19.1 ; \mathrm{df}=6 ; \mathrm{p}<, 003\right)$. Of the respondents who indicated to be non-religious, $39 \%$ had no partner, $41 \%$ had one partner and $20 \%$ had more than one partner in the past year. For those respondents who were Roman-Catholic, the percentages are $37 \%, 40 \%$ and $23 \%$ respectively and for the Protestants $44 \%, 39 \%$ en $17 \%$. On the other hand $72 \%$ of the respondents of Islamic religion had no partner, $19 \%$ had one partner and $9 \%$ more than one partner.

\section{Knowledge about Sexually Transmitted Diseases}

Subjects have on average correctly answered fourteen out of the 22 questions, while they did not know the answer to an average of five out of 22 questions and incorrectly answered an average of three out of 22 questions. By means of analysis of variance it has been verified whether there is an effect of the social 
demographic variables: gender, school attended, religion and origin on the correct level of knowledge. Only religion and origin demonstrate relationships with correct knowledge about STDs. Respondents who are of Dutch descent correctly answered an average of two questions more than respondents who are of Surinam descent $(F=4.7 ; p<.01)$. Slightly more than half of the respondents find their own level of STD-related knowledge poor to reasonably sufficient. Of the total study population $38 \%$ would like more information about STDs and $52.2 \%$ do not care to receive more information.

\section{Expected effectivenese of STD-preventive behavioure}

On average, the sexually active respondents have a total score of 45 on expected effectiviness of STD-preventive behaviours (minimum/maximum score: $13-65$, s.d. $=6.4)$. Women are on average $(M=47)$ more strongly convinced that the behaviour alternatives lead to STD prevention than men $(M=45)(F=9.0 ; p<0$ .5). For the other social demographic characteristics no difference in expected behaviour effectiviness was found.

In order to find out whether mutual factors underlie the expected effectiveness of STD-preventive behaviours, a factor analysis was carried out. Based on conceptual considerations and considerations concerning content we chose for a three-factor solution, namely: expected behaviour effectiveness of (1) condom use, (2) selective partner choice, (3) STD communication. A characteristic item for the factor of condom use is: "I can prevent venereal disease by always using condoms"; for the factor selective partner choice: "I can prevent venereal disease by carefully selecting my sexual partners" and for the factor STD communication: "I can prevent venereal disease by asking my sexual partner whether he or she has an STD". The internal consistency for these factors separately was: Cronbach's alpha $=0.82$, Cronbach's alpha $=0.57$ and Cronbach's $=0.60$, respectively.

Percelved Importance and annoyance of STD preventive behaviour On average, the respondents had a sumscore of 57 on the perceived importance of STD-preventive behaviour ( $\mathrm{min} / \mathrm{max}$ score: $25-74, \mathrm{s.d}=7.3$ ). The average score on perceived annoyance of STD-preventive behaviour was 45 ( $\mathrm{min} / \mathrm{max}$ score: $16-70 ;$ s.d. $=9.6$ ). On average, women find STD-preventive behaviour a little less important for themselves $(M=58)$ than men $(M=56) \quad(F=7.8 ; p<.005)$. Respondents who attend school part-time find STD-preventive behaviour on average less important for themselves $(M=53)$ than those who attend technical and vocational schools $(M=58)$, secondary modern school $(M=58)$ and grammar school $(M=59)$ ( $F=4.0 ; p<.005)$. Moreover, Roman-Catholics $(M=47)$ and Protestants $(M=49)$ find STD-preventive behaviour on average less annoying than the non-religious $(M=44)$ and Islamics $(M=43)(F=3.3 ; p<.05)$. For the other social 
demographic chracteristics no difference was found in perceived importance and perceived annoyance of STD-preventive behaviour. However, the respondents who are sexually inactive do find STD-preventive behaviour more important for themselves $(M=60)$ than those who are sexually active $(M=55)(F=21.0 ; p<.001)$. Separate factor analysis of the items on perceived importance and perceived annoyance results in the following 6 factors: (1) perceived importance and (2) annoyance of selective partner choice, (3) perceived importance and (4) annoyance of condom use and (5) perceived importance and (6) annoyance of STD communication. The internal consistency of these factors separately is for all scale higher than Cronbach's alpha $=0.65$.

\section{Previous STD-preventive behaviour}

On average, the respondents have a finalscore of 29 on previous STD-preventive behaviour ( $\min . / \max$. score: $12-48, \mathrm{~s} . \mathrm{d}=\mathbf{=} .0$ ). Women on average take STDpreventive actions slightly more often $(M=38)$ than men $(M=32)(F=4.4 ; p<.01)$. Likewise respondents of Islamic descent state more often to take STD-preventive actions $(M=37)$ than the irreligious $(M=34)$, the Roman-Catholics $(M=34)$ and Protestants $(M=35)(F=2.8 ; p<.05)$. On the other hand, the respondents who attend school part-time are less likely to take preventive actions in the future $(M=30)$ than those who attend technical and vocational schools $(M=36)$, secondary modern school $(M=35)$ and grammar school $(M=36)(F=5.7 ; p<.001)$.

\section{Determinants of the Intention to STD-preventive behaviour}

To study which above factors contribute significantly to the intention to perform STD-preventive behaviours, stepwise regression analyses were conducted for the group of the sexually inactive and the sexually active separately. Table 11 shows the results of the analysis for the group of the sexually inactive.

TABLE 11. AELATIONSHIPS BETWEEN EVALUATIONS, GENDER AND INTENTION TO STDPREYENTIVE BEHAVIOUR FOR THE SEXUALLY INACTIVES

\begin{tabular}{lrrr}
\hline PREDICTOR & $\boldsymbol{r}$ & beta & MR \\
\hline Perceived importance of STD communication & 0.51 & 0.51 & 0.51 \\
Perceived annoyance of selective partner choice & -0.40 & 0.27 & 0.57 \\
Gender $(0=\sigma ; 1=$ ?) & 0.23 & 0.19 & 0.61 \\
Perceived annoyance of STD communication & -0.40 & -0.19 & 0.63 \\
Perceived importance of selective partner choice & 0.45 & 0.20 & 0.66 \\
\hline
\end{tabular}

note: only significant predictors $(p<0.05)$ are shown.

explained variance $=43 \%$ 
Table 11 demonstrates that the intention to STD-preventive behaviour among the group of the sexually inactive can mainly be explained by the following factors: perceived importance of STD communication, perceived annoyance of selective partner choice, gender, perceived annoyance of STD communication and perceived importance of selective partner choice. By means of the beta coefficient, it has been established that especially persons who find talking about STD important, do not find selective partner choice annoying, women more likely than men, those who do not find talking about STD annoying and those who find selective partner choice important are more likely to take STDpreventive actions in future. Perceived importance and annoyance of condom use, expected effectiveness STD-preventive behaviours and the correct level of knowledge do not contribute significantly to the explanation of the variance in the intention to STD-preventive behaviour (F-to-enter probability is $p<.05)$. Table 12 shows the results of the analysis for the sexually active.

TABLE 12. RELATIONSHIPS BETWEEN PREVIOUS STD-PREVENTIVE BEHAVIOUR, EXPECTED BEHAVIOUR EFFECTIVENESS, EVALUATIONS, OENDER AND INTENTION TO STD-PREVENTIVE BEHAVIOUR FOR THE SEXUALLY ACTIVES

\begin{tabular}{llll}
\hline PAEDICTOR & & bola & MR \\
\hline Previout STD-preventive behaviour & & & \\
Expected behaviour effectiveness of STD communication & 0.73 & 0.73 & 0.73 \\
Gender $(0=d ; 1=$ ) & 0.38 & 0.23 & 0.76 \\
\hline
\end{tabular}

nole: only significant predictors $(p<0.05)$ are shown.

explained variance $=63 \%$

Table 12 shows that the most important contribution to the intention to STDpreventive behaviours ameng the group of the sexually active originates from previous STD-preventivg behaviour. Furthermore, beliefs about the expected effectiveness of STD communication and gender contribute significantly to the intention to perform STD-preventive action. The total explained variance was $63 \%$. It emerged that especially persons who now take preventive action, those who are convinced that talking about STD can prevent venereal diseases and women are more likely to take STD-preventive action in tuture. Perceived importance and annoyance of STD communication, perceived importance and annoyance of selective partner choice, perceived importance and annoyance of condom use, and correct knowledge do not contribute to the prediction of the intention to STD-preventive behaviour. 
To find out which variables play a role in previous STD-preventive behaviour of the sexually active, once again a stepwise multiple regression analysis was conducted (see Table 13).

TABLE 13. RELATIONSHIPS BETWEEN EXPECTED BEHAVOUR EFFECTIVENESS, EVALLLA TIONS, GENDER AND PREVIOUS STD-PREVENTIVE, BEHAVIOIUR FOR THE SEXUALLIY ACTIVES

\begin{tabular}{lrrc}
\hline PREDICTOR & & bela & MA \\
\hline Perceived importance selective partnerchoice & 0.52 & 0.52 & 0.52 \\
Perceived importance condom use & 0.42 & 0.33 & 0.68 \\
Perceived annoyance of STD communication & -0.27 & -0.29 & 0.613 \\
Gender (0=di, 1-8) & 0.24 & 0.18 & 0.70 \\
Porceived importance STD communication & 0.48 & 0.16 & 0.71 \\
\hline
\end{tabular}

note: only significant predictors $p<0.05$ are shown explained varlance $=51 \%$

Table 13 shows that perceived importance of selective partner choice, perceived importance of condom use, perceived importance and annoyance of STD communication and gender contribute most significantly to the prediction of current STD-preventive behaviour. In particular respondents who find selective partner choice and condom use important, those who do not find talking about STD annoying, women more likely than men, and those who find talking about STD important, are more likely to demonstrate STD-preventive behaviour. The correct level of knowledge, expectations about the behaviour effectiveness of: condom use, STD communication and selective partner choice, perceived annoyance of condom use and perceived annoyance of selective partiner choice play no role in current STD-preventive behaviour.

\subsection{Discussion}

The level of knowledge of the secondary school pupils is sufficient but not high. On average, only 14 out of the 22 questions were answered correctly. However, the level of knowledge of these adolescents can be compared with that of the Dutch population (Luykx ef al., 1987). The unknown level of knowledge, however, is relatively high among this group of pupils. Many adolescents would, therefore, like to receive more information about STDs. As is to be expected, knowledge about STDs does not contribute to intended STD-preventivei behaviour among secondary pupils. Women are more strongly convincecl of the behaviour effectiveness of several STD-preventive actions than men. Conside- 
ring the components from which the sumscore has been composed, it is mainly the expectation that a venereal disease can be prevented by condom use, talking about STDs and making a caretul selection of one's sexual partners. Most pupils do find the different STD-preventive actions important for themselves, but find it annoying at the same time. Particularly those who attend school part-time find STD-preventive actions less important for themselves. This could possibly be explained by the fact that their relationship is considered "steady". However, one-quarter of this group of adolescents has had more than one sexual partner in the past year. Moreover, the explanation could be that those who attend school part-time use one strategy consistently (for example having sex with a regular partner; serial monogamy) which is why they consider the other actions less important.

Half of the respondents strongly intend to take STD-preventive action. Particularly part-time pupils are less likely to demonstrate STD-preventive behaviour in the future. It is remarkable that respondents who are of Moroccan or Turkisch descent are more likely to take STD-preventive actions than those who are of Dutch and Surinam descent. However, especially pupils who are Islamic prove to be less sexually active. The stronger intention to take STD-preventive action has probably to do with a lack of sexual experience.

We established that the intention to STD prevention among sexually inactive pupils can chiefly be explained by the evaluatory aspects of STD-preventive behaviour, such as: perceived importance of STD communication, perceived annoyance of selective partner choice, perceived annoyance of STD communication and the perceived importance of selective partner choice (total explained variance $43 \%$ ). It is remarkable that the perceived importance and annoyance of condom use do not contribute at all to intended STD-preventive behaviour among sexually inactive pupils.

These findings implicate that the following elements should be stressed in STD prevention education for the sexually inactive: (1) discussing beliefs regarding the effectiveness and evaluation of selective partner choice and the effectiveness of STD communication in a sexual relationship and (2) empowering the youths with skills necessary for adequate STD communication. Also talking about STD preventive actions with sexual partners play an important role. Moreover, it is advisable, especially with regard to these pupils, to pay attention to the perceived importance and annoyance of condom use.

It was to be expected that the intention to STD-preventive behaviour among the groups of the sexually active can chiefly be explained by previous STD-preventive behaviour and by expected effectiveness of STD communication (total explained variance 63\%).

Based on these results it can be stated that among the group of the sexually active aftention should be paid to aspects of previous STD-preventive behavi- 
our. In education it should be found out which beliefs exist about selective partner choice, condom use and communication about STDs in a sexual relationship. Moreover, we advise to reduce the perceived annoyance of STDpreventive behaviour by increased habituation when teaching social skills (focused on these aspects of STD-preventive behaviour). Apart from the pupils who attend technical and vocational schools, secondary modern school and grammar school, those who attend school part-time form an important target group for health education in STDs and STD prevention.

Our research results partly support the setup of national campaigns by the Family Planning Clinic (Braeken, 1988). This campaign promotes communication between partners (how to make clear what you want) and making choices (have sex yes or no). The setup of this study implicates that the generalisability of the research results may be limited because the group of respondents investigated consisted only of pupils from a few schools in the Hague.

To summarise, we conclude that it is advisable to take the sexual experiences of those involved into account. Furthermore, the findings show that the intention to STD prevention can be promoted by focusing the intervention on making STDs a subject of discussion in a sexual relationship, and in addition emphasise to training social skills and talking about preventive behaviour. 


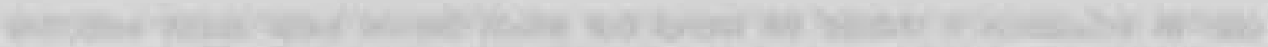

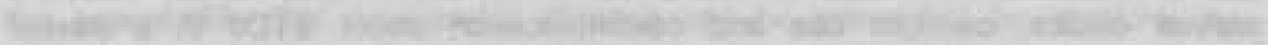

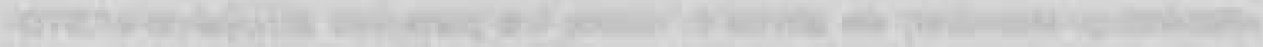

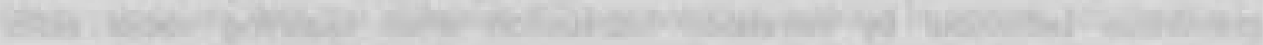

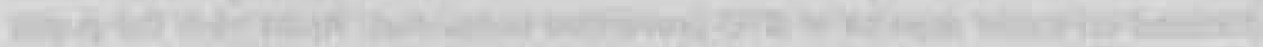

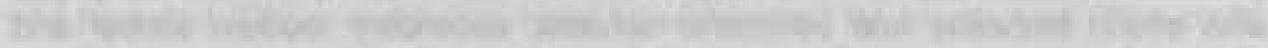

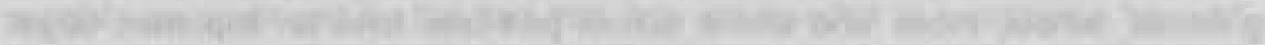

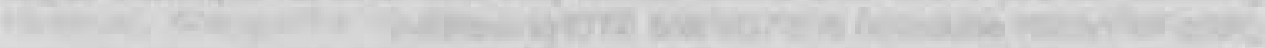

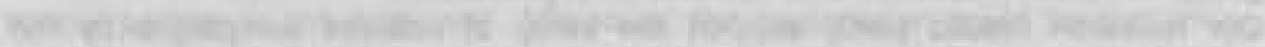

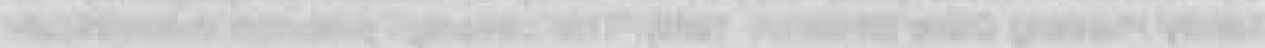

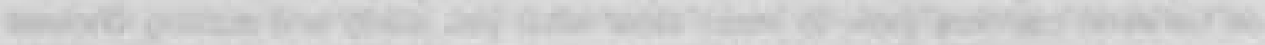

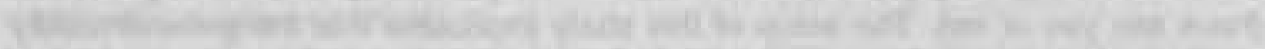

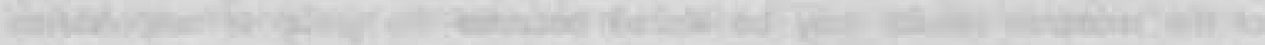

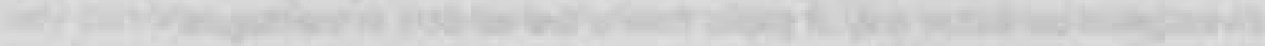

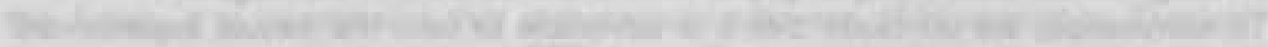

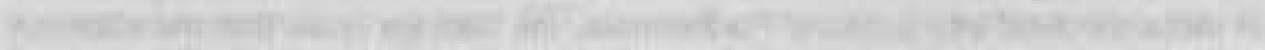
4.

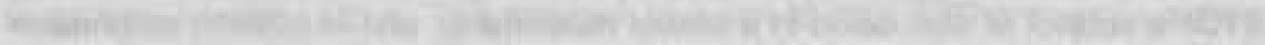

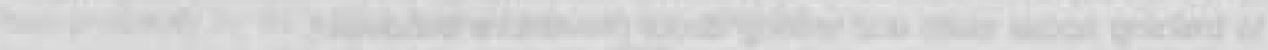

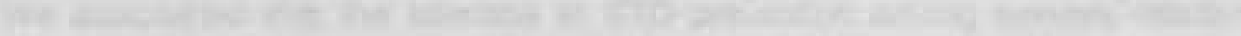

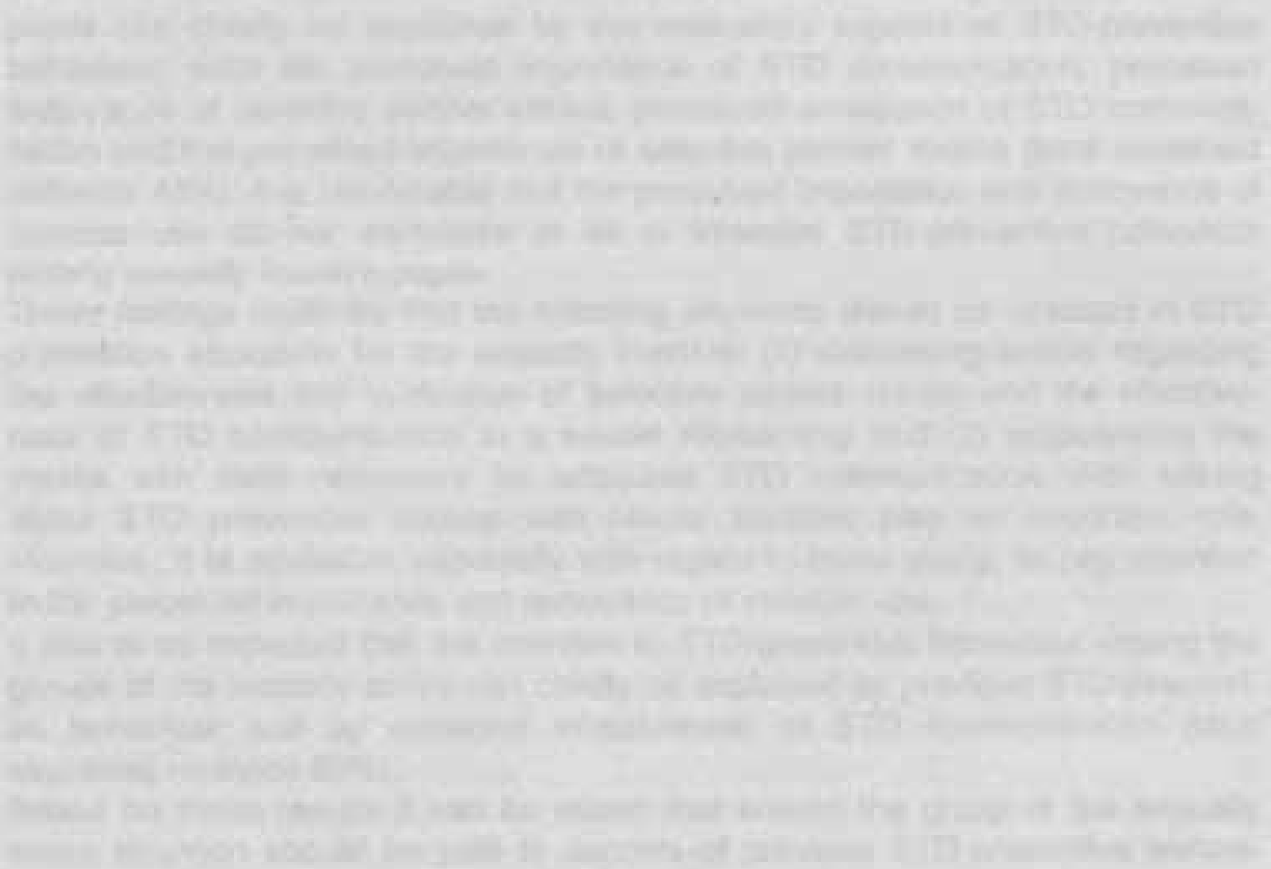




\section{PART III}

\section{EARLY DEIECTION}




\section{TRAS}

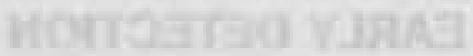




\section{STD-RELATED HEALTH BEHAVIOR: DETERMINANTS OF EARLY STD- DETECTION}

Submitted for publication as: Leenaars, P.E.M., Kok, G., Rombouts, R. STDrelated health behavior. determinants of early STD-detection.

\subsection{Introduction}

Sexually Transmitted Diseases (STDs) are communicable diseases that are estimated to occur frequently in the Netherlands. Apart from the epidemiological aspects of STDs, untreated STDs can have serious complications, such as Pelvic Inflammatory Disease (PID) and their late sequelae! (De Schrijver \& Meheus, 1990). Severijnen and Huisman (1992) assurne that two-thirds of all PID cases in the Netherlands are caused by STD pathogens. Registration of the Amsterdam Sentinel of General Practitioners shows that the incidence of PID in Amsterdam (a major city in the Netherlands) declined from 50 to 24 in 10,000 women in the period 1983-1990 (Coutinho, Rijsdijk, Van den Hoek \& Kuiper, 1992). Yet, in line with the findings of other researchers (Rolfs, Galaid \& Zaide, $1990)$ this decline was not present among females younger than 24 years old.

Besides vaccination for hepatitis $B$, no vaccins are available for the prevention of other STDs. Therefore, successful treatment depends on early detection of these infectious diseases (Cates \& Meheus, 1990). Yet, notwithstanding the existence of possible STD-related symptoms more than one-quarter of persons requesting a medical examination for a suspected STD waited more than four woeks before they sought medical treatment (Leenaars, Rombouts \& Kok, 1993).

In order to detect STD-related signs or symptoms at an early stage, STD selfexamination by persons reporting unprotected sexual intercourse with multiple sex partners may be one of the health-protective behaviours that can result in early disease detection. STD self-examination involves inspection of one's genitals for abnormal discharge, unpleasant smell, itching, burning or other irritations, pain or painful urination, genital warts, ulcerations and other visible abnormalities. Unfortunately, the symptoms of many STDs can be inconspicous, mild or even completely absent (asymptomatic), especially in women. Because the lack of symptoms is an unreliable guide to freedom from infection, periodic clinical examinations by persons performing high-risk sexual behaviour may also be seen as an important health-protective strategy to prevent the development of complications.

Until now no research has been carried out on the effectiveness of STD sellexamination and periodic clinical examinations woth regard to complications of STDs. Yet, research on other health-protective behaviours showed that selfexamination behaviour resulted in the detection of tumors at an earlier, more treatable stage (Tamburini, Massara, Bertario, Alberto \& Putio, 1981). Knowled- 
ge about determinants of STD self-examination and periodic clinical examinations is needed 80 that interventions to encourage health protective behaviour can be developed.

Various psychological theories of value expectancy and decision-makingl attempt to explain the adoption of health behaviours, for example: the Health Belief Model (HBM) (Maiman \& Becker, 1974; Rosenstock, 1974; Janz \& Becker, 1984), the Theory of Reasoned Action (Fishbein \& Ajzen, 1975; Ajzen \& Fisbein, 1980), Protection Motivation Theory (Rogers, 1983) and Bandura's Social Cognitive Learning Theory (SCLT) (Bandura, 1986). In a recent comparison of these models Weinstein (1993) stated that these theories (SCLT not included) emphasise beliefs about health hazards and health-protective behaviours and have many features in common, although the simularities are seldom recognised.

Considering the variables of interest in this investigation namely, STD selfexamination and periodic clinical examination, we believe that with the aim of developing a short questionnaire, the HBM model leads to more meaningful questions than other models. In addition, the idea that theories may not only be used to predict behaviour but also to guide recommendations for intervertions, supports the choice for the HBM model. The HBM-model (Rosenstock, 1974) assumes that people will be most likely to follow a health recommendation if they have an incentive or a motive to do so and if they hold four separate beliefs: (1) that they are vulnerable to the disease or to its consequences; (2) that the disease or its consequences have a serious negative impact on their lives; (3) that following a particular set of recommendations will be effective in reducing the severity of the disease and (4) that psychological and other barriers associated with following the health recommendation are outweighted by its benefits. The theory adds that this process is triggered by a cue to action, a signal that motivates health-protective behaviour. in their review of 46 ampirical studies in which the Health Belief Model was used, Janz and Becker (1984) found evidence that some of the HBM components (vulnerability, severity, benefits and barriers) contribute to the prediction of a wide range of health behaviours.

Despite the possible usefulness of STD self-examination and periodic; clinical examination, little research has been done to explore determinants of these early STD detection behaviours until now. Only one study (Simon \& Das, 1984) examined health beliefs in relation to the frequency of past asymptomatic check. ups for venereal disease and found that $10 \%$ of the variance in past asympto. matic check-ups was explained mainly by barriers to taking health action and by perceived vulnerability to venereal diseases.

In order to develop effective intervention programmes it is essential to get a better understanding of early STD detection behaviors. As part off a larger study designed to explore help-seeking behaviour, the present study was designed to 
address the lacunae in research on (determinants of) both STD self-examination and periodic clinical examination by persons reporting frequent unprotected intercourse with multiple sex partners. The specific aim of this study was to gain insight into the contribution of perceived severity, perceived vulnerability. benefits of and barriers to both behaviours, so that recommendations for the content of interventions can be made. We also investigated the effects of two sets of variables, not specificly incorporated in the HBM, that inay also influence intentions to early STD detection behaviours. The first selt reflects an evaluative approach of the recommended behaviours (direct attitude toward periodic clinical examination, direct attitude toward STD self-examination). Furthermore, the role of risk behaviour and previous STD detection activities (previous STD self-examination and previous periodic clinical examination) in intendecl STD self-examination and intended periodic clinical examination was explicitly investigated in this study.

\subsection{Method}

\section{Subjecte and procedure}

Ali subjects ( $\mathrm{N}=855)$ sought a medical examination for a suspected (new) STD. Patients visiting health services in eight tegions (major cities in the Netherlands) participated in the study and within each region subjects were recruited from an STD-clinic, a Family Planning Clinic, a General Practitioner (GP) and an independent dermatologist/venereologist. Individuals who were not proficient in Dutch and those who were attending for a check-up of an old STD, were excluded from the study. According to the information gathered from the healthcare providers a 92 percent response-rate $(\mathrm{N}=855)$ was obtained. The healthcare provider encouraged the patients to participate in the investigation and then gave instructions for answering the questionnaire. All participants completed a structured questionnaire after the individual consultation. Anonymity was guaranteed. At the moment of answering the questionnaire the respondents: were not informed about the results of the examination. The structured questionnaire was developed after a literature study and interviews with health providers and professionals in the field of STDs. During the interviews special attention was paid to the benefits of and barriers to medical treatment.

\section{Measures and data reduction}

This study involved the following questionnaires and variables:

- Sociodemographic variables: age, gender, education, urban versus rural recidence, sexual preference, professional prostitution, customer of prostitutes, sexual contact with anonymous partners;

- Risk behaviour was assessed by: 
the number of sexual partners in the past six months and type of sexual partner $(0=$ no sexual partner, $1=$ one regular or a new partner, 2 = one different sexual partner in addition to one regular partner (including one time visiting a prostitute), $3=$ more than two familiar sexual partners, $4=$ more than two anonymous sexual partners (including customers of prostitutes) and 5 = being a prostitute;

the frequency of condom use ( 0 =almost always, 1 =irregularly, $2=$ almost never).

Multiplying number (type) of sexual partners by the frequency of condom use results in an index for risk behaviour, indicating: $0=$ no risk behaviour to score $10=$ very high risk behaviour. The combination of response categories is not entirely mutually exclusive, but only reflects a global measure of risk behaviour;

- Intention to early STD-detection was assessed by:

- the intention to periodic clinical examination: I intend to have myself tested on STDs regularly (every three months);

. the intention to STD self-examination: 1 intend to examine my own genitals regularly on STD-related symptoms.

A 5-point scale with endpoints 'extremely unlikely' and 'extremely likely' (1-5) was used for each item $(r=0.46 ; p<.001)$.

- Previous STD-detection behaviours (habits) were assessed by two items $(r=0.51 ; p<.001)$ :

. previous STD self-examination, such as: I examined my genitals on STDrelated symptoms. A 4-point scale with endpoints 'never' and 'very often' (1-4) was used for each item;

- previous periodic clinical examination was measured by asking the respondent how many times they visited a physician for a suspected STD in the past two years: never, once, two to four times and four to eight times and more than eight times.

- Direct attitude to periodic clinical examination and the direct attitude to STD self-examination were measured by four items, assessing perceived importance and perceived annoyance of periodic clinical examination $(r=0.51 ; p<.0-$ 01) and STO self-examination $(r=0.50 ; p<.001)$, respectively. For example: how important do you personally find examining your own genitals on STDrelated symptoms? A 5-point scale with endpoints 'extremely unimportant' and 'extremely important' was used for each item.

Questions concerning Health Belief Model components:

- A Perceived Severity Scale: developed to assess perceived severity of ten possible consequences resulting from having an STD. A 5-point scale with endpoints 'disagree entirely' and 'agree entirely' (coded 1-5) was used for each item, for example: if I have an STD, I will feel dirty. Factor analysis resulted in three groups of components (health beliefs): 
- anticipated affective reactions: assessed by evaluating five potential feelings (guilt, dirtiness, embarrassment, uncertainty, sickness) when having an STD (Cronbach's alpha $=0.78$ );

- anticipated consequences on relationships: measured by evaluating three potential consequences of STDs on their relationships (getting into arguments, getting divorced, feeling disloyal) (Cronbach's alpha $=0.81$ );

- anticipated physical consequences: measured by asking the level of agreement with two possible bodily consequences (infertility and skininjury). Because of their low association $(r=0.14 ; p<.01)$ these variables were analysed separately.

- A Perceived Invulnerability Scale: developed to assess perceived invulnerability to contracting an STD due to several (ineffective) risk-reducing behaviours. A 5-point scale with endpoints 'strongly disagree' and 'strongly agree' (coded 1-5) was used for each item, for example: I thought I had a low risk of contracting an STD, because $1 /$ my sexual partner use(s) the pill/oral contraceptives (Cronbach's alpha $=0.63$ ). Factor analyses with varimax rotation resulted in three factors (health beliefs) corresponding with three different (ineffective) risk reducing practices, namely:

- perceived invulnerability due to safe-sex practices: measured by two items: condom use and safe-sex $(r=0.37 ; p<.001)$;

- perceived invulnerability due to careful choice of partners was measured by two items: selective partner choice and careful partner choice ( $r$ $=0.32 ; p<.001$ ):

- perceived invulnerability due to lay health beliefs (ineffective risk-reducing practices): measured by four items (cleaning genitals, using pill as contraception, healthy life style, treated for an STD before (Cronbach's alpha $=0.74$ ).

- Perceived benefits of and barriers to medical treatment were measured on a 5-point scale by asking the patient's levell of agreement with the existence of sixteen service attributes, for example: if I seek a medical examination here, I will get adequate medical treatment. Factor analysis resulted in four factors:

- Quality of medical care. four items consisted off getting: professional medical treatment, personal treatment, adequate medical treatment and rapid cure (Cronbach's alpha $=0.80$ );

- time investment of an STD examination. mealsured by four items (travelling time, waiting time, interruption of (daily) work and walk-in consulting hours (Cronbach's alpha $=0.74$ );

- anonymous treatment facilities. assessed by three items: the general practitioner, health insurance company and family and friends do not get informed of the STD consultation (Cronbach's alpha reliabilty $=0.71$ ).

Because the fourth factor was not an interpretable one (Cronbach's alpha $=0.11$ ) we analysed the rest of the service attributes (five) separately. 
- Cue to action. assessed by two questions asking if the respondents obtained information from either leaflets and posters, or radio and television. A 3-point scale with endpoints 'not at all' and 'very much' was used for each item $(r=0.48 ; p<.001)$.

\section{Data analyale}

All data analyses were performed by standard statistical procedures of the SPSS/PC+ version 3.0 (Norusis, 1988).

Univariate analyses of variance and Scheffe multiple comparison tests were used to assess differences in risk behaviour, direct attitude, intention to STD self-examination and intention to periodic clinical examination between men and women and respondents with a low, middle and high educational background respectively. Pearson correlations were calculated between age, urbanisation of residential town, health beliefs, benefits of and barriers to medical treatment, cues to action, direct attitudes, previous behaviours and the separate intentions (only $1>0.20 ; p<.05$ are reported).

Next, to assess the relative predictive power of variables related to intention to STD sell-examination and intention to periodic clinical examination, two multiple regression analyses (stepwise entry) were carried out. A two-sided significance level of $5 \%$ was used for all analyses.

\subsection{Results}

\section{Description of respondents}

A total of 855 persons completed the questionnaire. The mean age was 29 years (s.d. $=9$ years) and $59 \%$ were men. The majority of the respondents had a low $(32 \%)$ or middle $(43 \%)$ educational background; almost half of the patients (48\%) were working class employees (unskilled and skilled labourers) and $22 \%$ were students. Almost $40 \%$ of the respondents lived in a big city (inhabitants > 200.000 ). The majority had heterosexual relationships, $12 \%$ reported homo- or bisexual relationships, $6 \%$ of the respondents were prostitutes and $11 \%$ were customers of prostitutes.

\section{Intention to early STD-detectlon and soclal demographic characterlstics}

About one-quarter of the sample reported having the intention to periodic clinical examination to some degree, $38 \%$ showed a more ambivalent intention and the other respondents had no intention to periodic clinical examination at all. In addition, $58 \%$ claimed, to scme degree, the intention to STD self-examination, while one-quarter reported a more ambivalent intention and $16 \%$ claimed no Intention to STD self-examination at all.

Co-variance analysis with prostitution as a covariate and gender as an independent variable revealed that irrespective of being a prostitute, women $(M=3.9)$ 
are more likely than men to seek periodic clinical examinations $(M=3.3).(F=7 ; p$ $<.001)$. In addition, respondents with a low education are more likely $(M=3.5)$ than those with middle $(M=3.0)$ and high education $(M=2.9)$ to seek periodic clinical examinations $(F=21.3 ; p<.001)$. No relationships were found between the other social demographic characteristic and intended periodic clinical exarninations, and intended STD self-examination.

\section{Risk behavlour and Intentlon to early STD detection behaviour}

About $20 \%$ of the respondents $(N=169)$ showed a score ' 0 ' on the index for risk behaviour, indicating they almost never had unprotected sexual intercourse with a new partner in the preceding six months (minimal risk behaviour); $45 \%$ had an index score 1 or 2, indicating they had unprotected sexual intercourse with one casual pertner or prostitute (moderate risk behaviour); $28 \%$ had an index score 4 or 5 and $7 \%$ had a score 8 to 10 , which means they have had sexual intercourse with more than two (anonymous) sexual partners, while irreqularly or never using condoms (high risk behaviour) $(M=2.6 ; s . d .=2.4)$. No relationships existed between risk behaviour and intended STD self-examination, and intended periodic clinical examinations.

Previous STD detection behaviour and direct attitude to STD detection Forty percent of the respondents stated that they often examine their own genitals on STD-related symptoms. Three-quarters of the respondents evaluated STD self-examination for themselves as (very) important, while most of them perceived STD self-examination not at all annoying.

About half of the respondents $(46 \%)$ claimed they never had a medical examination for venereal diseases, $28 \%$ said they had visited a physician for a suspected STD once before and one-quarter indicated they had venereal disease check-ups at least twice in the past two years. One-third of the respendents evaluated periodic clinical examinations for themselves as (very) important and forty percent expressed more ambivalent feelings. Furthermore, $40 \%$ found periodic clinical examinations (very) annoying.

\section{THE HEALTH BELIEF MODEL}

In this section we will first examine interrelations among factors (health beliefs) within each belief set (perceived severity and perceived invulnerability). Elsewhere, we gave a detailed description of differences in health beliefs between men and women, low, middle and high educated respondents, and between respondents living in villages, small towns, medium-sized towns and big cities, respectively (Leenaars, Rombouts \& Kok, 1993). Furthermore, relationships are calculated between health beliefs, benefits of and barriers to medical treatment, cues to action, previous early detection behaviours and attitudes (we will only present significant relationships). 
Rolationships within bellef sets, between bellef sots, prevlous behaviours and attltudes

A strong relationship was found between anticipated affective reactions and anticipated consequences for relationships $(r=0.53 ; p<.001)$. Moderate to small relationships existed between perceived invulnerability due to lay health beliefs and both invulnerability due to careful partner choice $(r=0.25 ; p<.001)$ and anticipated affective reactions $(r=0.21 ; p<.001)$. The other relationships were all r<.20;p>0.05. In addition, strong relationships ( $>0.30 ; p<.01)$ were found between previous STD self-examination, attitude to STD self-examination and previous periodic clinical examination, the attitude to periodic clinical examination, respectively. Finally, rather small relationships ( $r>0.20$ and $r<0.23 ; p<.05$ ) were found between invulnerability due to lay health beliefs and invulnerability due to safe sex practices on the one hand and direct attitude to periodic clinical exarnination on the other. Relationships between other health beliefs, benefits of and barriers to medical treatment and direct attitude to periodic clinical examination were absent. No relationships existed either between attitude to STD selfexamination and the separate health beliefs.

\section{Cues to action}

Almost all respondents indicated they obtained information about STDs from leaflets or posters or from television or radio. No relationships were found between intention to STD self-examination and periodic clinical examination on the one hand and being exposed to cues to action on the other.

\section{Determinante of Intentlon to STD self-examination}

In order to examine which above variables contribute significantly to the intention to perform STD self-examination a stepwise multiple regression analyses was carried out. The results of the analysis (pearson correlations, partial correlation coefficients, beta's and multiple R) are presented in Table 14.

TABLE 14 RELATIONSHIPS BETWEEN PREMOUS STD SELF-EXAM, DIRECT ATTITUDE, MEALTH BELIEFS AND INTENTION TO STO SELF-EXAMINATION

\begin{tabular}{lcccc}
\hline PREDICTORs & r & partial r & bot & MR \\
\hline Diroct attitude & 0.68 & 0.55 & 0.68 & 0.68 \\
Previgus STD self-examination & 0.55 & 0.29 & 0.25 & 0.72 \\
Antic. relational consequences & -0.05 & -0.12 & -0.06 & 0.72 \\
Invulnerability due to safo sox & 0.16 & 0.08 & 0.05 & 0.72 \\
Anticipated affective reactions & 0.01 & 0.09 & 0.08 & 0.72 \\
\hline
\end{tabular}

Explained variance $=52 \%$ 
Stepwise regression analysis of intention to STD self-examination on direct attitude, previous STD self-examination, components of perceived severity and perceived invulnerability showed that $52 \%$ of the variance in intended STD selfexamination was mainly explained by the direct attitude toward STD selfexamination and previous STD self-examination behaviour.

\section{Determinante of Intention to perlodic clinical examination}

In order to assess the contribution of variables in predicting intention to periodic clinical examinations a second stepwise multiple regression analyses was carried out. The results of the analysis (pearson correlations, partial correlation coefficients, beta's and multiple R) are presented in Table 15.

TABLE 15. RELATIONSHIPS BETWEEN GENDER, EDUCATION, PREVIOUS PERIODIC EXAMS, HEALTH BELIEFS, DIRECT ATTITUDE AND INTENTION TO PERIODIC CLINICAL. EXAMINATION

\begin{tabular}{lcccc}
\hline PREDICTORS & r & partial r & beta & MR \\
\hline Direct attitude & 0.72 & 0.60 & 0.70 & 0.73 \\
Previous poriodic clinical exam & 0.47 & 0.20 & 0.12 & 0.74 \\
Invuln. due to lay health beliefs & 0.29 & 0.18 & 0.21 & 0.75 \\
Invuln. due to salo sex & 0.21 & 0.08 & 0.10 & 0.75 \\
Education & -0.21 & -0.06 & -0.21 & 0.75 \\
Anticipated infertility & 0.10 & 0.07 & 0.07 & 0.75 \\
Inviln due to partner choice & -0.07 & -0.07 & -0.09 & 0.75 \\
\hline
\end{tabular}

Explained varlance $=56 \%$

Stepwise regression analysis of intention to periodic clinical examination on gender, education, direct attitude, previous periodic clinical examinations, components of perceived invulnerability and perceived severity and benefits of and barriers to medical examination showed that the direct attitude toward periodic clinical examinations and previous periodic clinical exarninations accounted for most of the variance in intended periodic clinical examination.

Gender, some components of perceived severity and perceived invulnerability and benefits of and barriers to medical treatment did not contribute significantly to intended periodic clinical examinations.

\subsection{Discusslon}

This study reveals that STD self-examination is not a regular practice at all among most individuals seeking a medical examination for STDs, while a considerable amount of these persons are at increased risk of contracting STDs. It is remarkable that no relationship existed between risk behaviour and 
both intended STD self-examination and intended periodic clinical examination. The findings support the idea of discussing the importance of STD self-examination behaviours as well as periodic clinical examination during consultating hours with those persons showing high risk sexual behavior. This idea is also supported by the finding that, not suprisingly, previous behaviour is one of the strongest predictors of both behavioural intentions and actually may reflect behaviour stability over time.

Irrespective of prostitution, females are more likely than males to seek periodic clinical examinations. The notion that females are more concerned with health and health behaviour (Hibbard, 1984) and that they report a greater tendency to consult physicians for hypothetical health problems was also reported by others (Cleary, Mechanic \& Greenley, 1982).

Apart from the results of Simon and Das (1984) little is known about behaviourial determinants of STD self-examination as well as periodic clinical examination. Yet, many investigations have been carried out on several other self-examination behavlours, such as breast self-examination (Shepperd, et al., 1990; Champion, 1990), skin examination behaviour (Cody \& Lee, 1990) and testicular self-examination (Brubaker \& Wickersman, 1990). In these studies past frequency of breast self-examination, barriers, health motivation, control, being taught by a doctor, benefits and susceptibility (Champion, 1990), barriers, age, history of harmless breast problems and knowledge (Sheppercl et al., 1990) and barriers, previous experience with skin cancer (Cody \& Lee, 1990) and attitude, self-efficacy, knowledge and subjective norm (Brubaker \& Wickersman, 1990) predicted the recommended health behaviour.

In our study intention to STD self-exarnination was mainly explained by the direct attitude toward STD self-examination as well as the past performance of the recommended behaviour. Furthermore, we found that perceptions of severity and invulnerability accounted for a negligible proportion of the variance in intention to STD self-examination. Even $\mathrm{H}$ thess variables are subject to change, the results of this study indicate that manipulation of these variables will not have any effect on intention to STD self-examination. We therefore recommend that further research on STD self-examination should start with an exploration of sailient beliefs (benefits and barriers) related to perceived importance and perceived annoyance (direct attitude) of STD self-examination. In addition, self-efficacy expectations (Bandura, 1982) and social norms (Ajzen \& Fishbein, 1980) as well as other nonmotivational factors may also influence the performance of STD self-examination.

With regard to periodic clinical examination, perceived vulnerability and perceived severity accounted for a small proportion of the variance, while benefits of and barriers to medical treatment explained next to nothing. However, these determinants again fade in comparison to the contributions of the direct attifude toward periodic clinical examination which accounted for most of the variance in 
intention to periodic clinical examination. In accordance with our results and the findings of Simon and Das (1984) as well, we may conclude that in further research a detailed examination of beliefs contributing to perceptions of importance and annoyance of periodic clinical examination has to be done. Interesting enough, the barriers and benefits index (as measured by possible negative and positive service attributes of STD health care facilities) do not act as benefits of and barriers to periodic clinical examination. The absence of any predictive value of perceived benefits of periodic clinical examination may be improved by measuring perceptions of non-health consequences as well as health related concerns. In addition, exploration of other facilitating factors should be done in future research.

As opposed to assumptions of the HBM model in this study perceived invulnerability explained only a small amount of variance in intended periodic clinical examination. Furthermore, the results show that several other components of the HBM model do not contribute to the prediction of intention to early STD detection behaviours at all. We, therefore, may conclude that the utility of the HBM model in explaining early STD detection behaviours is limited. Yet, the selection and operationalisation of measures should also be considered as reasons for the failure of the research to find results. In this study we measured perceived invulnerability in terms of the consequences of various (perceived) risk reducing practices, while in most other studies vulnerability is generally assumed to reflect beliefs about the likelihood that a negative health outcome will occur by performing the undesirable behaviour.

The results of the present study do support the applicability of an evaluative approach to periodic clinical examinations. Yet, while intention tu periodic clinical examination was predicted by the subjects' direct attitude toward periodic clinical examinations, no relationhip was found between beliefs about treatment outcomes or other service attributes (barriers and benefits) and direct attitude to periodic clinical examination. One explanation for this finding is that we did not measure the most sailient beliefs about the outcomes of the recommended behaviour. This means that further investigation into intention to periodic clinical examination must start with assessing sailient beliefs of periodic clinical examination among persons performing high-risk sexual behaviour, for example recurrent attenders of an STD-clinic.

An important methodological issue involves the cross sectional nature of the study and consequently the prediction of intentions, while we did not measure actual behaviour. This means that we could not test the extent to which intentions predicted the separate behaviours. Ajzen and Fishbein (1980), however, have demonstrated that if specific conditions are fulfilled, intentions predict behaviour in a wide range of behaviour domains. In addition, while a longitudina! investigation into early STD detection behaviours should be recommended, such research is almost impossible to carry out among persons suspecting an STD. 
Another methodological problem is revealed from the fact that the selection of respondents leads to a deficit in generalisation of the findings to the population of STD patients. For example, including ethnic minority groups to the sample selected, may have repercussions on the research findings.

In summary, encouraging the regular practice of STD self-examination and periodic clinical examinations by educetion and individual counselling seems to be important in order to prevent STD-related complications in infected individuals as well as preventing further transmission of STDs. The variables mainly associated with increased intention to early STD-detection appear to be those of attitudes toward the proposed behaviour and habits. These dimensions are important in raising the performance of early STD-detection behaviour in persons with high-risk sexual behaviour. Further research should focus on beliefs influencing the attitude and means of changing these attitudes. 


\section{SEEKING MEDICAL CARE FOR A SEXUALLY TRANSMITTED DISEASE: DETERMINANTS OF DELAY BEHAVIOUR}

Published as: Leenaars, P.E.M., Rombouts, R., Kok, G. (1993). Seeking medical care for a Sexually Transmitted Disease: determinants of delay behaviour. Psychology and Health, 8, 17-32.

\subsection{Introduction}

Sexually Transmitted Diseases (STDs) are defined as a group of communicable diseases which are transferred predominantly by sexual contact (WHO, 1986). Until now the number of pathogens known to be sexually transmitted has increased to more than twenty. Because of a considerable amount of underreporting (estimated from $60 \%$ to $95 \%$ in different regions of the Netherlands), the national notification data for gonorrhea and syphilis only allows for estimations of true incidence of several STDs (Miltenburg, Paalman \& Reus, 1985). For example, the reported incidence of gonorrhea in the Netherlands in 1990 was 3,666 cases (GHI, 1991). In general, trencis have shown a significant decline in traditional venereal diseases Nan de Laar, Van den Hoek, Pickering, Van Griensven, Coutinho \& Van der Water, 1990). However, De Schrijver and Meheus (1990) have recently stated that these classical bacterial diseases have been replaced (in industrialized countries) by both bacterial and virai syndromes associated with sexually transmitted organisms, which are usually more difficult to identify, to treat and to control.

Apart from the public health aspects of STDs, untreated STDs can have serious complications and late sequelae in infected individuals, such as: salpingitis, sequelae of Pelvic Inflammatory Disease (PID) (for example: ectopic pregnancy and infertility), sequelae of urethritis and epididymitis, and cancers of the cervic, vulva, penis and anus associated with the Human Papilloma Virus (De Schrijver \& Meheus, 1990). So, not only the epidemiological impact but also the medical consequences and economic costs of treating STD-related complications confirm the relevance of early STD-detection and proper medical treatment of patients and their sexual partners.

At present there are no accurate estimates either of the annual number of physician consultations by persons reporting STD-related symptoms, or of the number of persons who do not obtain any medical treatment for their symptoms potentially attributable to an STD. Yet, STD-related symptoms can be relatively mild or inconspicous (for example: abnormal discharge, unpleasant smell, itching, burning or other irritation, pain or painful urination, genital warts, ulcerations or other visible abnormalities), and they might even be completely absent. Meanwhile, an immediate response to STD-related symptoms can contribute greatly to disease prevention. Orduna (1980) suggested, on the basis 
of a study on gonorrhea transmission that if the duration of symptoms for each heterosexual patient was reduced by one day, the number of reported cases of gonorrhea would decline by approximately eight percent. Unfortunately, a common response to symptoms and disease is to wait and see if symptoms persist, worsen, or subside (Suchman, 1965). There is evidence that this appears to be true for STDs as well (CDC, 1980; Stadlander \& Kok, 1989). Data from the Centers for Disease Control (CDC) in the United States indicated that men with primary syphilis delayed obtaining medical treatment on average twelve days after the initial appearance of their lesion (CDC, 1980). This delay behaviour varied with the educational background of the patient.

An increasing realisation of a number of non-medical factors influencing patients' perception and decision to obtain medical care (such as: the manner in which persons monitor their bodies, define and interpret their symptoms, take remedial action and utilise various sources of help, including the formal health care system) has resulted in a more detailed examination of the problem of underutilisation (Mechanic, 1982). In his study on women's treatment decisions with regard to STDs, Harrisson (1982) found that the reason why women did not obtain medical treatment for genital symptoms was partially explained by the classifying of these symptoms as normal. When women were uncertain about symptoms they stated that this uncertainty motivated them to obtain medical treatment. However, only $45 \%$ reported seeking medical treatment promptly.

In this study we are particularly interested in the extent of delay behaviour in persons reporting STD-related symptoms and the determinants of this delay behavlour. Delay behaviour refers to the time spent between perceiving physical symptoms potentially attributable to an STD (STD-related symptoms) and health care utilisation. Many aspects of delay behaviour are suitable for investigation with components of the Health Belief Model (HBM), originally developed by Hochbaum, Kegeles, Leventhal and Rosenstock (Rosenstock, 1974; Janz \& Becker, 1984). In their review of 46 studies which have used the HBM, Janz and Becker (1984) found evidence that some of the HBM components are important contributors to the prediction of health behaviour. With regard to STDs only one study (Simon \& Das, 1984) has used the HBM model in order to assess health beliefs in relation to the frequency of past asymptomatic STD check-ups. The results showed that $10 \%$ of the variance in past asymptomatic check-ups was explained mainly by barriers to taking health action and susceptibility to STDs. Apart from Harrisson's (1984), no other research into determinants of delay behaviour has boen carried out among persons suspecting an STD.

As part of a larger study designed to explore help-seeking behaviour, the present study examines determinants of delay behaviour in persons with STDrelated symploms. Special attention is paid to social demographic characteristics, high-risk sexual behaviour and Health Belief Model components that may' 
contribute to variations in delay behaviour. It is important to note that delay behaviour in persons with STD-related symptoms can result in the development of complications and late sequelae. So, it is assumed that HBM-determinants of delay behaviour are equal to HBM-determinants of STD-related health behaviour aimed at preventing complications.

\subsection{Methods}

\section{Procedure and sample}

All subjects $(\mathrm{N}=855)$ had attended a physician for a suspected (new) STD. Patients visiting health services in eight regions (major cities in the Netherlands) were selected as the eligible study population and within each region participants were selected from: (1) an STD-clinic; (2) a family planning clinic; (3) a general practitioner. Individuals who were not proficient in Dutch and those who were attending for a check-up of an old existing STD, were excluded from the study. According to the information gathered from the health care providers an overall response-rate of 92 percent $(\mathrm{N}=855)$ was obtained.

Health care providers encouraged their patients to participate in the investigation and then provided instructions for answering the questionnaire. All subjects completed a structured questionnaire after the individual consultation. Anonymity was guaranteed. A total of 242 respondents (28\%) reported no STD-related symptoms at all, but were advised to seek medical treatment by their sexual partner or other health care providers. Because of the central theme of this (sub)study, which is exploration of delay behaviour in respondents with STDrelated symptoms, persons without STD-related symptoms and those who did not have sexual intercourse in the preceding six months $(\mathrm{N}=28)$ were excluded from the study. All analyses were therefore based on the remaining respondents ( $N=585)$.

\section{Measures}

This study involved the following questionnaires and variables:

- sociodemographic variables: age, gender, education, urbanisation of residential town, sexual preference, prostitution as profession, customers of prostitutes, history of STD consultations (recurrent attenders).

- Delay behaviour. respondents reporting STD-related symptoms were asked how long they have been experiencing these symptoms: (1) one week, (2) two weeks, (3) two to four weeks and (4) longer than four weeks.

- High-Risk Sexual Behaviour was measured by asking:

- the number of sexual partners in the past six months $\quad(1=$ one regular partner, 2 = one different sexual partner in addition to a regular parther and $3=$ more than two different sexual partners); 
the frequency of sexual intercourse with anonymous partners ( $1=$ never, 2=irregularly or always);

- the frequency of condorn use ( $1=$ never, 2 =irregularly, $3=a$ always).

- Symptom IIIness Questionnaire: respondents who reported STD-related symptoms were asked which of the following symptoms they experienced: abnormal discharge, itching, pain, ulcers, genital/anal warts or other symptoms.

Questionnaires concerning Health Belief Model components, consisted of:

- Perceived Severity Scale: developed to assess perceived severity of 10 possible consequences resulting trom having an STD. A five-point scale ranging from 'disagree entirely' and 'agree entirely' was used for each item. Cronbach's alpha reliability for the total scale (10 items) was 0.79 . Factor analysis resulted in three groups of factors:

anticipated affective reactions: measured by asking respondents to ovaluate five potential feelings (guill, dirtiness, embarrassement, uncertainty, sickness). Cronbach's alpha reliability was 0.78 ;

anticipated consequences on relationships: measured by asking respondents to evaluate three potential consequences for their relationships (getting into arguments, getting divorced, feeling disloyal). Cronbach's alpha reliability was 0.81 ;

anticipated physical consequences. measured by asking the level of agreement with two possible bodily consequences (infertility and skininjury). Because of their low association $(r=0.14)$ these two variables were analysed separately.

- Perceived Invulnerability Scale: developed to assess perceived invulnerability to contracting an STD due to several (ineffective) risk-reducing behaviours. A five-point scale ranging from 'strongly disagree' and 'strongly agree' was used for each item. Cronbach's alpha reliability for the total scale (8 items) was 0.63 . Factor analyses resulted in three factors corresponding with three different risk-reducing practices, namely:

perceived invulnerability due to lay health beliefs (ineffective risk-reducing practices): measured by four items (cleaning genitals, using the pill as contraception, healthy life style and having been treated before for an STD). Cronbach's alpha reliability was 0.74 ;

perceived invulnerability due to safe sex practices: measured by two items (condom use and safe sex) $(r=0.37 ; p<.001)$;

perceived invulnerability due to careful choice of sexual partners: measured by two items (selective partner choice and careful partner choice) ( $r=0.32 ; p<.001$ ).

- Perceived benefits of and barriers to medical treatment were measured on a five-point scale by asking the patient's level of agreement with the existence of sixteen service attributes. If some service attributes are expected to exist 
they may act as barriers, while most of them may act as benefits. The following service attributes are questioned: professional medical treatment, proper medication, rapid cure, personal treatment, travelling time, waiting time, interruption of work, walk-in consulting hours, anonymous treatment facilities (family and friends, GP and the insurance company are not informed of the STD examination), evening consulting hours, option of a male or female physician, free medical care, professionals have a judgmental attitude to STDs and, lastly, one stays uncertain of test results for a long time.

- External Cues to Action: measured by asking whether respondent3 obtained information from either leaflets and posters, or television and radio. In addition, respondents were asked if they discussed their suspicion of having an STD with someone else and if so, whether they were advised to obtain medical treatment by their partners, family/friends or by another health care provider (provider-referred contact tracing) or it they came of their own accord.

- Internal cues to action were separated in:

- the attribution of symptoms to sexual behaviour: assessed by asking the respondents on what level (four-point scale) their sexual behaviour influenced them to seek medical treatment.

lack of need for a consultation: assessed by asking the respondents' level of agreement with two misconceptions (such as: the symptoms will disappear spontaneously) $(r=0.64 ; p<.001)$.

- personal characteristics: measured by asking the respondents' level of agreement with four possible reasons for their delay behaviour (delay as a habit, embarassment or shame of an STD, anticipated painful examination and seeking the right physician).

\section{Data analysls}

Data analyses were performed by standard statistical procedures of the SPSS/PC+ version 3.0 (Norusis, 1988). All data analyses were based on 585 respondents with STD-related symptoms. According to the extent of delay behaviour in respondents with STD-related symptoms, four delay behaviour groups were distinguished: group 1, respondents who waited one week; group 2, respondents who waited two weeks; group 3, respondents who waited between two to four weeks; group 4, respondents who delayed longer than four weeks.

Chi-square tests of independence were used to assess the relationship between social demographic characteristics and delay behaviour. Because of interrelations between the individual dependent variables, multivariate analyses of variance (MANOVA) were used to determine if the four delay behaviour groups differed from each other on a combination of dependent variables. Afterward, univariate F-tests were used to assess which of the combined dependent variables were related to delay behaviour. Scheffé multiple comparison tests 
were used to determine which of the four groups was responsible for the difference in mean scores on the dependent variables. Discriminant analysis was applied to make a linear combination of the observed variables with the ultimate goal of distinguishing optimally between respondents belonging to two extreme delay behaviour groups (group 1 versus group 4). For all analyses, a two tailed significance level of five percent was used.

\subsection{Results}

Description of respondente with STD-related eymptoms

A total of $\mathbf{5 8 5}$ persons having had sexual intercourse in the preceding six months and reporting STD-related symptoms, completed a questionnaire. The mean age of the patients was 29 years (s.d. $=9$ years) and $64 \%$ were male. The majority of the respondents had a low $(31 \%)$ or middle $(46 \%)$ educational background. More than half of the patients $(66 \%)$ were lower class employees; and $22 \%$ were students. Almost $45 \%$ of the respondents lived in a big city (inhabitants $>200.000$ ). The majority of the respondents $(90 \%)$ reported a heterosexual preference, $6 \%$ were prostitutes and $14 \%$ were customers of prostitutes. About half of the respondents (46\%) claimed they never had an STD examination, $28 \%$ had visited a physician for an STD once before and about one-quarter had at least two STD check-ups in the past two years.

\section{Patlent characterletice and delay behaviour}

In this section we will examine relationships between delay behaviour and the following patient characteristics: type of symptoms experienced, social demographic variables, high-risk sexual behaviour.

\section{Reported symptoms and delay behaviour}

In this sample $77 \%$ experienced secretion or itching, $8 \%$ pain, $11 \%$ ulcerations and $4 \%$ genital warts. The respondents were asked how long they had been experiencing these symptoms before they decided to consult a physician. Almost half of the respondents $(\mathrm{N}=238)$ waited less than one week, $19 \%$ waited one to two weeks $(\mathrm{N}=113), 13 \%$ waited two to four weeks $(\mathrm{N}=76)$ and $27 \%$ walted more than four weeks $(N=158)$. No relationship was found between the type of symptoms experienced and delay behaviour.

\section{Soclal demographic characteristics and delay behaviour}

Several chi-square analyses were carried out to discover whether deley behaviour is related to social demographic characteristics. Gender differences were found in the level of delay behaviour $\left(x^{2}=13.6 ; \mathrm{di}=3 ; p<.001\right)$. Women more often showed two weeks, two to four weeks and moret than four weeks of delay behaviour and men were more likely to seek medicall assessment within one 
week after noticing STD-related symptoms. Detailed examination of the delay behaviour in women yielded a relationship between educational level and delay behaviour $\left(x^{2}=18\right.$; df $\left.=6 ; p<.05\right)$ as well as between their cohabiting situation and delay behaviour $\left(x^{2}=14.5 ; d f=6 ; p<.05\right)$. Women with a middle educational background more often showed two to four weeks delay behaviour and women with a low or high education more often came within one or within two weeks. Women cohabiting with their regular (sexual) partner more often showed more than four weeks delay behaviour, whereas women living on their own or with their parents more often came within one week and within two weeks. No relationships were found between delay behaviour in women on the one hand and age, recurrent visitors, urbanisation of residential town and female prostitution on the other hand.

A relationship existed between urbanisation of residential town and delay behaviour $\left(x^{2}=34 . ; d f=9 ; p<.001\right)$. Fespondents living in a village more often showed more than four weeks delay behaviour than those living in small, medium sized or big cities. Respondents living in small towns reported two to four weeks delay behaviour more often than those others living in medium sized or big cities. A closer examination of the delay behaviour among respondents living in a village revealed a relation between gender and delay behaviour $\left(\alpha^{2}=33 ; d f=2 ; p<.001\right)$. Men living in a village more often showed more than four weeks delay behaviour, while women living in a village more often consulted a physician within one week. There was no relationship between delay behaviour of respondents living in a village and age. The minimum expected cell frequencies were too small to test interdependency between other social demographic variables and delay behaviour among village inhabitants.

Sexual preference was also related to delay behaviour $\left(\alpha^{2}=9.2 ; d f=3 ; p<.05\right)$. Respondents with a homosexual preference were more likely to visit a physician within one week and heterosexual respondents were more likely to show two to four weeks delay behaviour.

No differences were found between the four delay behaviour groups with respect to the distributions of educational background and age. Also, no relationship was found between the number of previous STD consultations (recurrent attenders) and delay behaviour nor between choice for a health care professional and delay behaviour.

High risk sexual behavlour and celay behavlour

Knowledge of one's own high risk sexual behaviour may influence immediate help-seeking behaviour. Forty two percent of the respondents had sexual intercourse with one permanent partner in the preceding six months, $20 \%$ had one different sexual partner in addition to one permanent partner, and $38 \%$ had sexual intercourse with more than two different partners. Women more often stated that they have had sexual intercourse with one permanent partner and 
men more often claimed they had sexual intercourse with more than two different partners $\left.Q^{2}=26 ; d f=2 ; p<.001\right)$. No relationship was found between the number of sexual partners and delay behaviour. When looking at condom use in respondents with more than one partner $(\mathrm{N}=341)$, we found that $24 \%$ never used condoms, $57 \%$ indicated they irregularly and $19 \%$ said they always used condoms during sexual intercourse. No relationship was found between condom use and delay behaviour in respondents with more than one sexual partner. About one third of the respondents (32\%) reported having had sexual intercourse with anonymous partners. Having had sexual intercourse with anonymous partners was related to delay behaviour $\left(x^{2}=14 ; d f=3 ; p<.001\right)$. Respondents having had sexual intercourse with anonymous partners were more likely to obtain medical treatment within one week as opposed to those who stated that they had never had sex with anonymous partners. No relationships were found between visitors of prostitutes and delay behaviour nor between being a prostitute and delay behaviour.

THE HEALTH BELJEF MODEL

In this section we will first examine relationships between social demographic characteristics (gender and urbanisation of residential town) and the more general individual health beliefs, followed by a description of interrelations among factors within each set of beliefs and relationships between the various HBM-components.

\section{Percelved severity}

Analysis of perceived severity due to anticipated affective reactions showed that more than half of the respondents reported to some degree feelings of guilt (57\%), embarassment (62\%), uncertainty $(79 \%)$ and dirtiness (75\%). Sixty-six percent believed themselves to be ill. When having an STD, $57 \%$ of the respondents expected consequences for their relationships and $70 \%$ believed that an STD may result in infertility and skin-injury. Women expected stronger than men that a STD may result in infertility $(F=15.1 ; p<.001)$, while men had a stronger belief than women that an STD may result in skin-injury ( $F=9.2 ; p<.001)$. Furthermore, there appeared to be a relationship between gender and anticipated consequences for relationships ( $F=27 ; p<.001)$. Men had a stronger expectation than women that a STD may have consequences for their relationship.

Village inhabitants expected consequences for their relationship more often than respondents living in small, medium sized and big cities ( $F=4.3 ; p<.005)$, whila no relation existed between anticipated affective reactions, anticipated skininjury, anticipated infertility on the one hand and urbanisation of residential town on the other. 


\section{Perceived Invulnerablilty}

Regarding perceived invulnerability due to risk-reduction behaviours, about three-quarters of the respondents indicated that they felt themselves invulnerable due to their careful choice of partners and due to their safe sex-practices. Furthermore, 25\% fett themselves invulnerable to a STD due to lay health beliefs. With regard to the individual lay health beliefs we found that $33 \%$ of the respondents still believe that they are unable to contract a STD because they wash their genitals after sexual intercourse. Only the perception of invulnerability due to careful choice of partners was related to gender $(F=5.1 ; p<.01)$, indicating that women felt themselves more invulnerable due to their careful choice of partners than men.

No relationships existed between urbanisation of residential town and percelved invulnerability due to lay health beliefs and due to safe sex practices, respectively. However, a relationship was found between urbanisation of residential town and perceived invulnerability due to careful choice of partners $(F=4.9 ; p<.0$ 01). A Scheffé multiple comparison test revealed that respondents living in villages and those living in big cities perceived themselves more invulnerable due to careful choice of partners than those living in small and medium sized cities.

\section{Percelved benefits and barrlers}

In response to questions on expected benefits of and barriers to medical trealment, one-quarter agreed that the following service attributes exist: waiting in a waiting room for a long time, spend much time commuting, interrupling their daily routine life, evening consulting hours, choice for male or female physician and also that the health professionals show a judgmental attitude to their sexual behaviour. Almost half of the respondents believed that there are walk-in consulting hours, free medical care and anonymous treatment facilities (the general practitioner and the health insurance company will not be informed of the STD examination). In addition, more than half of the respendents expressed agreement with the following service attributes: quick cure, receiving professional treatment, being prescribed proper medication and get personal treatment. Furthermore, the majority of the respondents believed that their friends and family would not find out about the STD consultation.

\section{External cues to action}

The majority of the respondents $(83 \%)$ stated that they came of their own accord, while $17 \%$ claimed that the reason they obtained medical treatment was because they were warned to do so by their sexual partner $(10 \%)$ (patient referred contact tracing) or a health care provider (7\%) (provider referred contact tracing). Furthermore, one third of the respondents acquired information from leaflets or posters before seeking medical treatment and more than half of the respondents talked with someone else about the possibility of having a STD. 
A relationship was found between gender and being advised by others $\left(x^{2}=24\right.$; $d i=1 ; p<.01$ ), indicating that women were more likely to be advised by their sexual partner or health care providers and men were more likely to obtain medical treatment of their own accord. No relationship existed between acquiring information from leaflets and posters on the one hand and gender and urbanisation of residential town on the other hand.

\section{Internal cues to action}

Internal cues to action were measured in terms of lack of need for consultation, the attribution of STD-related symptoms to specific sexual behaviours and personal characteristics. About forty percent of the respondents expressed a high need for a consultation, indicating that they did not expect their syrnptoms to disappear spontaneously, while almost half of the respondents stated that their sexual behaviour did not play a role in the decision to seek medical treatment. No relationship existed between lack of need for a consultation and gender or urbanisation of residential town. Homosexual men $\left(\chi^{2}=6.8 ; d f=1 ; p\right.$ <.001) and respondents with more than two sexual parthers $\left(x^{2}=92 ; d f=2 ; p<.001\right)$ more often attributed their STD-related symtpoms to their sexual behaviour than heterosexual men and respondents having had sexual intercourse with one or with two sexual partners.

When asking patients why they did not seek medical treatment immediately after perceiving eymptoms, about half of the respondents stated that delay behaviour as a 'habit' in seeking medical care $(50 \%)$ and embarassment $(40 \%)$ were reasons for their delay behaviour. In addition, $20 \%$ claimed that finding the right physician was a reason for their delay behaviour.

Village inhabitants more often reported searching for the right physician as a reason for delay behaviour than respondents living in cities $\left(\chi^{2}=10 ; p<.01\right)$ as well as embarassment was more often a reason for their delay behaviour $\left(x^{2}=10 ; p<.01\right)$. No relationships were found between delay as a habit, embarassement, searching for the right physician and gender.

\section{Relations between Health Belief Model varlables}

In order to assess relationships within each set of belief and between sets of beliefs, Pearson correlations were calculated. A strong relationship was only found between anticipated affective reactions and anticipated consequences for relationships $(r=0.53 ; p<.001)$. Moderate to small relationships were found between perceived invulnerability due to lay health beliefs and both invulnerability due to careful choice of partners $(r=0.25 ; p<.001)$ and anticipated affective reactions $(r=0.21 ; p<.001)$. In addition, the attribution of STD-related symptoms to specific sexual behaviour was moderately related to anticipated affective reactions $(r=0.20 ; p<.001)$ and to anticipated consequences for relationships $(r=0.24 ; p<.001)$. Moreover, a small relationship existed between lack of need for 
a consultation and delay behaviour as a habit $(r=0.20 ; p<.01)$. The other Pearson correlations were rather weak $(r<0.20)$ or completely absent.

The Health Bellef Model and delay behavlour

Because of the nominal measures and the skewed frequency distributionsi of some dependent variables, we first used crosstabulation and chi-square analyses to examine relationships between some of the internal and external cues to action, and delay behaviour. Chi-square anslyses revealed thet respondents with two to four weeks and those with more than four weeks delay behaviour more often claimed that they were advisecl to obtain medical treatment by their (sexual) partrier, while respondents with one week and those with two weeks delay behaviour more often stated that they came of their own accord $\left(x^{2}=24 ; d f=3 ; p<.001\right)$.

In addition, a relationship existed between delay as a habit and delay behaviour $\left(x^{2}=11.9 ; d f=3 ; p<.001\right)$, indicating that respondents with two weeks and those with two to four weeks more often claimed than those who came within one week and those who waited more than four weeks that delay as a habit was a reason for their delay behaviour. Delay behaviour was also related to embarassment $\left(\chi^{2}=8.6 ; d f=3 ; p<.05\right)$ and searching for the right physician $\left(\chi^{2}=9.2 ; d f=3\right.$; $p<.05)$, indicating that respondents with two weeks and those with two to four weeks and those with more than four weeks delay behaviour more otten claimed that embarassement and searching for the right physician were reasons for their delay behaviour.

Multivariate analyses of variance (MANOVA) with four levels of delay behaviour were carried out to determine relationships between the HBM components and delay behaviour. The results showed significant differences between the four delay behaviour groups in a combination of health beliefs. (Wilk's lambda =.82; p<.001).

\section{Main effects of delay behaviour on HBM variables}

As presented in Table 16 a relationship existed between delay behaviour and the following variables: anticipated infertility, anticipated skin-injury, beliefs about the existence of three service attributes (walk-in consulting hours, rapid cure and being prescribed proper medication), lack of a need for a consultation, the attribution of STD-related symptoms to specific sexual behaviour, pressure of the partner and acquiring mass media information. A more detailed analysis of the data with Scheffe multiple comparison tests revealed that respondents with two weeks delay behaviour had a stronger expectation that a STD may result in infertility than those with one week delay behaviour. Furthermore, respondents with two to four weeks and those with more than four weeks delay behaviour had a stronger expectation that an STD may result in skin-injury than those with one week delay behaviour. Also, respondents with two to four weeks delay 
behaviour were more likely to believe that they have to make an appointment first than respondents who came within one week. In addition, respondents who sought medical treatment within one week believed stronger that they would be prescribed proper medication and that they would be cured quickly than those who waited more than four weeks. Respondents who sought medical treatment within one week experienced a stronger need for a consultation than those with two to four weeks and more than four weeks delay behaviour. Also, respondents with more than four woeks delay behaviour related their symptoms less often to specific sexual behaviour than those with one week, two weeks and two to four weeks delay behaviour. In addition, respondents with two to four woeks delay behaviour experienced more pressure from their partner(s) and family than those who came within one week and those who sought medical treatment within two weeks. Lastly, respondents with two to four weeks and those with more than four weeks delay behaviour were more likely to acquire information from leaflets and posters than those who came within one week.

In Table 16 mean scores and standard deviations of the dependent health beliefs, cues to action and only the significant service attributes are presented for the seperate delay behaviour groups, together with the F-ratios.

TABLE 16. MEANS AND STANDARD DEVLATIONS FOR THE DELAY BEHAVOUR GROUPS, TOGETHER WITH F-VALUES

\begin{tabular}{|c|c|c|c|c|c|}
\hline 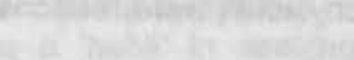 & & DELAY & EHAVIOUR & & \\
\hline 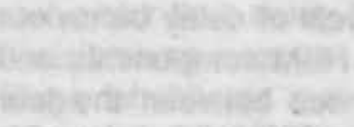 & $\begin{array}{l}\text { one } \\
\text { week } \\
\text { (1) }\end{array}$ & $\begin{array}{l}\text { two } \\
\text { weeks } \\
\text { (2.) }\end{array}$ & $\begin{array}{l}\text { two-four } \\
\text { weekss } \\
\text { (3) }\end{array}$ & $\begin{array}{l}>\text { four } \\
\text { weeks } \\
\text { (4) }\end{array}$ & 60 \\
\hline variables & $n=23 ?$ & $n=114$ & $n=76$ & $n=158$ & i-ration \\
\hline pere. Invuln. dus to & & & & & \\
\hline lay nealth betiefs & $2.3(0.93)$ & $2.0(0.86)$ & $2.3(0.93)$ & $2.1(0.87)$ & 2.3 \\
\hline Bafts sox practicen & $3.1(0.96)$ & $3.3(0.99)$ & $3.3(1.00)$ & $3.1(0.92)$ & 1.7 \\
\hline carotul cholce for partiners & $3.2(0.98)$ & $3.2(0.82)$ & $3.4(1.00)$ & $3.2(0.94)$ & 1.0 \\
\hline Perc. eoverity & & & & & \\
\hline antic. aff, reactions & $3.0(1.00)$ & 3.1 (0.96) & $3.2(1.00)$ & $3.0(1.00)$ & 0.2 \\
\hline antic. relational cons. & $2.7(1.00)$ & $2.7(1.10)$ & $2.4(1.20)$ & $2.4(1.00)$ & 1.2 \\
\hline entic, infertility & $3.1(1.10)$ & $3.5(1.1)^{i}$ & $3.4(1.00)$ & $3.2(1.00)$ & $4.0^{\circ-m}$ \\
\hline antic. skin-injury & $3.0(1.00)$ & $3.4(0.90)$ & $3.5(1.10)^{\prime}$ & $3.4(1.10)^{\prime}$ & $5.2^{-1 *}$ \\
\hline Benefite a Barriere & & & & & \\
\hline walk in consulting hour & $0.5(0.89)$ & $1.0(0.96)$ & $1.3(0.91)^{\prime}$ & $1.1(0.99)$ & $4.1^{\mathrm{m}}$ \\
\hline qukk cure & $2.0(0.81)^{4}$ & $1.8(0.87)$ & $1.9(0.81)$ & $1.7(0.85)$ & $4.5^{m+*}$ \\
\hline proper medication & $2.2(0.66)^{4}$ & $2.1(0.68)$ & $2.1(0.68)$ & $2.0(0.73)$ & $2.8 *$ \\
\hline
\end{tabular}




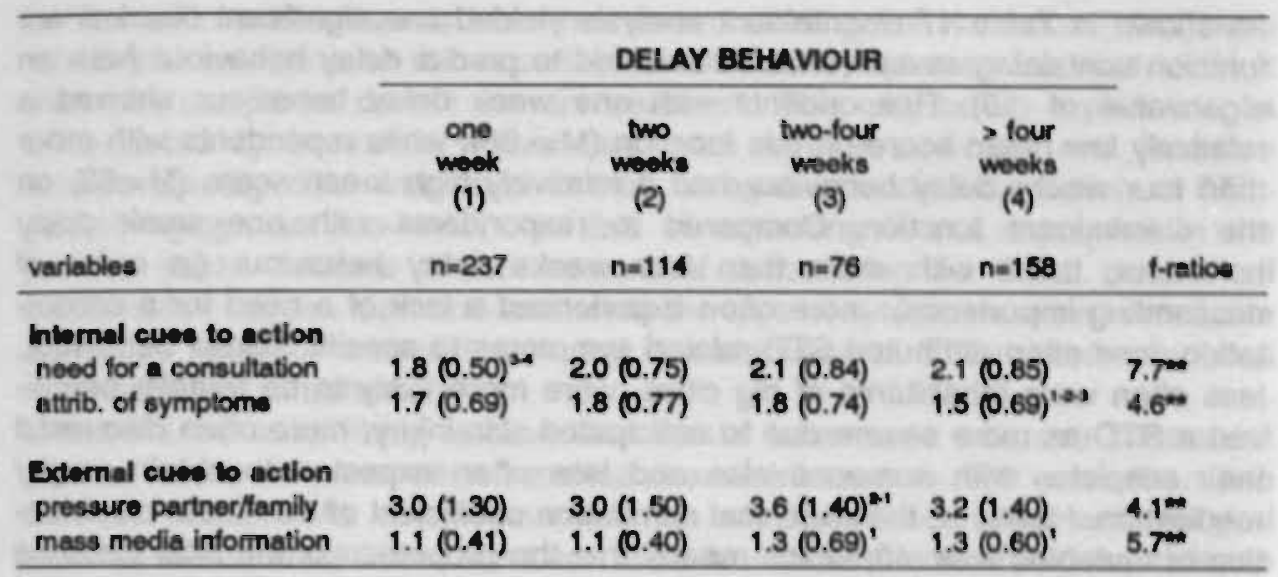

note: "p<.01; "p<.001; Subscripts $(1-4)$ indicate differences between pairs of means. The number refers to the different groups.

Interaction offects of gender and delay behaviour on health beliofe No interaction effects existed between delay behaviour, gender and the separate HBM components.

\section{Comparlsons among subgroups}

To gain a clear understanding of the variables which may explain the long delay behaviour in respondents with STD-related symptoms and to asses the predictive power of the established significant variables, we compared respondents in the extreme delay behaviour groups: respondents with one wiek delay behaviour $(\mathrm{N}=238)$ were compared to respondents who reported more than four weeks delay behaviour $(\mathrm{N}=158)$ (see Table 17).

TABLE 17. STANDARDIZED DISCAIMINANT FUNCTION COEFFICIENTS OF VARIABLES USEED TO PREDICT DELAY BEHAVIOUR (TWO GROUPS)

\begin{tabular}{|c|c|c|}
\hline $\begin{array}{l}\text { ORDER } \\
\text { OF ENTRY }\end{array}$ & PREDICTOR & $\begin{array}{l}\text { DISCAIMINANTT } \\
\text { COEFFICIENT }\end{array}$ \\
\hline 1 & leck of need for consultation & -35 \\
\hline 2 & symptoms aftributed to specifie sexual behaviour &. .35 \\
\hline 3 & urbanisation of residential lown &,- 40 \\
\hline 4 & gender & 47 \\
\hline 5 & anticipated skin-injury & .33 \\
\hline 6 & partner pressure & .28 \\
\hline 7 & being prescribed proper medications & -.28 \\
\hline$x=2$ & cononical conrolation & .40 \\
\hline
\end{tabular}


As shown in Table 17 discriminant analysis yielded one significant discriminant function containing seven variables selected to predict delay behaviour (with an eigenvalue of .19). Respondents with one week delay behaviour showed a relatively low mean score on this function $(M=-.35)$, while repondents with more than four weeks delay behaviour had a relatively high mean score $(M=.53)$ on the discriminant function. Compared to respondents with one week delay behaviour, those with more than four weeks delay behaviour (in order of descending importance): more often experienced a lack of a need for a consultation, less often attributed STD-related symptoms to specific sexual behaviour, less often were inhabitants of big cities, were more likely to be female, perceived a STD as more severe due to anticipated skin-injury, more often discussed their suspicion with someone else and less often expected to obtain proper medication. However, the canonical correlation coefficient of this linear combination of variables was .40 , which means that the proportion of the total variance in the discriminant scores explained by differences in variables between the two groups is rather small, namely $16 \%$. Classification of delay behaviour based on these seven variables led to $70 \%$ correctly classified cases. Almost $73 \%$ of the respondents with one week delay behaviour were correctly classified (prior probability .58) and $64 \%$ of the respondents with more than four weeks delay behaviour (prior probability .42).

\subsection{Dlscusslon}

The principal aim of this study was to assess determinants of delay behaviour in Individuals suspecting an STD. About one-quarter of these persons waited longer than four weeks before obtaining medical treatment. Compared to findings in similar studies on delay behaviour in STD patients (Stadlander \& Kok, 1989; CDC, 1980) our results and the findings of Harrisson (1982) suggest that delay behaviour in individuals with STD-related symptoms is much higher than expected.

The results of the present study underline the notion that certain subgroups of the population, especially women with a middle educational background and those who live together with one permanent partner may need to be specially targeted for delay behaviour related interventions. On the contrary, in a recent review of physician utilization, Kessler (1986) concluded that men and women with life threatening acute health problems do not differ in speed for an initial contact. In regard to STDs as less threatening diseases, one might expect that a possible explanation for the women's delay behaviour could be that women perceive themselves less vulnerable to an STD due to their careful choice of partners. The findings from this study demonstrates that perceived invulnerability due to careful choice of partners did not lead to longer delay behaviour in women as compared to men. A second possible explanation for the delay 
behaviour in women sterns from the research findings of Harrisson (1982) who found that women who are uncertain about their symptoms also tend to think that their symptoms do not warrant treatment. This may be an explanation as STD-related symptoms in women are more atypical and mild and therefore women actually differ from men in their recognition and interpretation of STD. related symptoms. However, this line of reasoning is not supported in the present study. At least at the moment of consultation we found no marked differences between men and women in the type of symptoms experienced, the perceived need for a consultation and the attribution of STD-related symptoms to specific sexual behaviour.

Women were more likely to have had sexual intercourse with one permanent partner and men more often reported sexual intercourse with more than two different partners. As others have pointed out (Thompson, 1983) self reported rates of extramarital intercourse are likely to underestimate the true incidence since non-monogamy is still widely disapproved. So it may be assumed that at least some of the women in this sample were infected through their non-monogamous (permanent) partner; this may explain the prolonged delay behaviour in women. The fact that men were more likely to expect consequences for their relationships supports the idea that their permanent female partner is uninformed about their extra marital sexual behaviour. Further research is necessary to assess determinants of delay behaviour in women, taking into account symptom processing and taboos concerning non-monogamous relations more briefly.

Village inhabitants, particularly men, were more likely to show delay behaviour compared to persons living in cities. While embarassment and searching for the right physician were more often mentioned by these subjects as reasons for their delay behaviour these only lead to two weeks delay behaviour. They also more often related consequences for their relationships to the delay behaviour, which may indicate that the taboo concerning STDs acts as a barrier to prompt help-seeking behaviour, particularly in this group.

Perceptions of vulnerability are related to preventive behaviours (Bauman \& Siegel, 1987). Taking protective action against STDs may lower peoples' vulnerability. From this point of view, one could argue that perceptions of invulnerability due to risk- reducing (ineffective) practices may explain the long delay behaviour. The present data, however, do not support the idea that low perceptions of vulnerability prolong delay behaviour. Although levels of knowledge about STDs (not including AIDS) are generally high (Luykx, Marsman \& Riit, 1987; Armonker, 1980), widespread misconceptions or gaps in infor!nation have been identified (Kroger \& Wiesner, 1981; Leenaars, Rombouts, Lammerdin \& Zwetsloot, 1990). In this sample too, about one third of the respondents still believed that washing or cleaning their genitals after sexual intercourse could prevent them from contracting an STD. 
Heterosexual men and persons who 'know ' their sexual partners were more likely to delay than homosexual men and persons who have had sex with anonymous partners. The underestimation of ones chances, the tendency to perceive oneself es somehow immune to health risks, which Weinstein (1980) defined as unrealistic optimism, was recently investigated in persons requesting a medical examination in an STD clinic (Hooykaas, Van der Pligt, Van Doornum, Van der Linden \& Coutinho, 1989). With regard to STDs, all samples showed significant levels of unrealistic optimism for contracting gonorrhea, syphilis and HIV Nan der Velde, Van der Pligt \& Hooykaas, 1992). In our sample, perceptions of invulnerability may be explained by unrealistic optimism as a result of overestimating the efficacy of ones preventive activities. Evidence from other empirical studies suggests that peoples' theories about disease causation not only contain ideas about the typical circumstances likely to produce an illness but also stereotypes or images of those types of people who are particularly vulnerable to a specific disease (Calnan, 1987). For example, Van der Pligt (1991) concluded that AIDS and STDs were seen as the two (out of twelve) health risks that were most clearly associated with specific groups. These extreme prototypes or stereotypical judgments may produce unrealistic optimism, thereby explaining prolonged delay behaviour in heterosexual men and in persons who know their sexual partners. Nevertheless, having sex with more than one different partner without using a condom leads to a higher perception of vulnerability, but not to more immediate help-seeking behaviour.

Most persons perceive an STD as moderately severe. Only perceived severity due to anticipated skin-injury leads to more immediate help-seeking behaviour, while perceptions of severity due to anticipated infertility is stronger in respondents with two weeks and two to four weeks delay behaviour. Despite their experience with STD health care, recurrent attenders with STD-related symptoms do not obtain medical treatment sooner than those who seek treatment for the first time. That previous delay behaviour, delay as a habit, predicts subsequent action is supported in several investigations on delay behaviour (Timko, 1987). Based on these findings it may be worth noting to consider the consultation for STDs as 'a teachable moment'; special attention can be given to primary as well as to secondary preventive strategies, such as prompt help-seeking behaviour.

Despite the overwhelming attention paid to sexual behaviour, HIV-infection and other STDs, embarassment about having an STD is still present in the Dutch population. Moreover, in spite of the existence of special STD clinics (their principal aim is to eliminate any barrier between the patient and his or her access to specialist diagnosis and treatment), embarassment still is a barrier to seeking immediate medical treatment for at least some individuals. From the results presented with regard to searching for the right physician, it seems necessary to give detailed information about the existence and approach of special STD- 
clinics. Moreover, giving more publicity to these special clinics may reduce delay due to searching for adequate medical treatment. From the sixteen service attributes, only expectations about: the existence of walk-in consulting hours, being prescribed proper medications and being cured quickly more often resulted in immediate help-seeking behaviour. From this point of view it is desirable to work with walk-in consulting hours and physician-designed protocols to ensure prescribing proper medications and quick cure. As regards the overall perceived importance of all of the special service attributes (Leenaars, Rombouts, Lammerdin \& Zwetsloot, 1992) it is possible that they serve as necessary conditions for STD help-seeking behaviour.

Persons with long delay behaviour more often reported that they obtained medical treatment for an STD after they were advised to do so by their (sexual) partner. Partner notification, in this case patient-referred contact tracing, ought to be encouraged by the health care provider, whereby clear instructions must be given about the importance of warning all sexual partners immediately and motivating them to seek medical treatment promptly. Believing that the syrnptoms will disappear spontaneously as well as not attributing STD-related symptoms to specific sexual behaviour were also reasons for delay behaviour. One might recommend that patients should be taught how to recognize STDrelated symptoms as a signal to immediate help-seeking behaviour. Furthermore, it is also important to stress that STDs may be asymptomatic, so patients should be encouraged to obtain medical treatment after having unprotected sexual intercourse with someone who is suspected of having an STD.

When taking the results of the discriminant analysis into account it must be concluded that the predictive power of several health beliefs, expectations of getting proper medications and pressure by the partner was rather small. While the various components of the Health Belief Model have helped us to organize the data, its use to predict delay behaviour in the context of STDs appears to be limited. For example, only anticipated infertility, anticipated skin-injury and internal cues to action are marginally related to delay behaviour. These results are supported by the findings of Simon and Das (1984) who found that the HBM accounted for only $10 \%$ of the variance in previous asymptomatic check-ups for venereal diseases. This is, however, not surprising given the inconsistency in findings and low explained variance in preventive behaviours reported across studies thus far (Rosenstock, Stretcher \& Becker, 1988).

Perhaps, the retrospective nature of the present study, the heterogenous research population and the type of behaviour predicted (actual delay behaviour in the context of STDs) have also weakened the findings. Moreover, since sexual decision-making occurs in a broader context of a sociocultural systern (Darrow \& Siegel, 1990) it seems important to take into account many other factors, for exarnple: stigma related coping patterns (denial and misconceptions), the interpersonal nature of STD-disease causation, abnormal illness 
behaviour (venerophobia versus perceiving a STD as a minor risk of a particular lifestyle), maturational constructs, homosexuality and personality problems.

To conclude, the results of this study show that a persuasive communication system urging immediate help-seeking behaviour should be targeted at changing several cognitions. In addition, we recommend the introduction of novel, previously non-salient cognitions. Such cognitions might emphasize the value of having an STD diagnosed quickly and professionally. From these findings we may also conclude that the utility of the Health Belief Model is limited in explaining delay behaviour in individuals reporting STD-related symptoms. 


\section{SERVICE ATTRIBUTES AND THE CHOICE OF STD HEALTH SERVICES IN PERSONS SEEKING A MEDICAL EXAMINATION FOR AN STD}

Published as: Leenaars, P.E.M., Rombouts R., Kok, G. (1994). Service attributes and the choice of STD health services in persons seeking a medical examination for an STD, Social Science and Medicine, 8 (2), 363-371.

\subsection{Introduction}

Treatment of Sexually Transmitted Diseases (STDs) is a way to prevent them from being transmitted any further. In order to facilitate patients' help-seeking behaviour, free and anonymous access to diagnosis and treatment of venereal diseases are recognised as important services to control STDs (Catterall, 1984). National efforts to control STDs are strongly influenced by the complexily of the health care system in the Netherlands (Leenaars, Oliehoek \& Schrijvers, 1991). In the Netherlands, one can usually obtain diagnosis and treatment for an STD from three different types of institutions. The formal state of affairs is that persons who suspect an STD go to see a general practitioner (GP) for medical care. Diagnosis and treatment is carried out by the general practitioner concerned, or the patient can be referred to an independent specialist or a specialist in a hospital. The health insurance company pays for the visit, diagnostic examinations and prescriptions.

Since 1976, the department of Welfare, Public Health and Culture (MVC) has subsidised out-patient STD clinics in the four largest cities (Amsterdam, Rotterdam, The Hague and Utrecht), which have high quality facilities for examination, diagnosis, treatment and contact tracing, and offer free access and anonymous medical care to all residents of the Netherlands who: (1) are not insured; (2) do not have a general practitioner; or (3) wish to remain anonymous because of the taboo on STDs. The patient does not need a letter of referral from a GP in order to obtain treatment from these clinics. Some clinics have an appointment system, while others are walk-in clinics. In order to make these "low-threshold facilities" possible in the remaining towns, the "VoMiL regulation" was set up (named after the then department of public health and environmental affairs). Persons who do not wish to visit a general practitioner can, by virtue of this regulation, be referred to an independent dermatologist or a dermatologist in a general hospital by a social nurse working at a Municipal Health Service (MHS). In addition to these low-threshold out-patient STD clinics and institutions with VoMil-regulations, nine MHSs have been allowed to offer, under certain conditions, free and anonymous diagnosis and treatment for venereal diseases, since 1985. Apart from the above health providers and institutions, there are a number of Family Planning Clinics in the Netherlands that deal with STD prevention and 
curation. In these institutions, however, the patients themselves have to pay for their diagnosis and treatment.

The above description shows that the structure of curative STD services is rather vague. This complicated structure may have repercussions on persons who seek medical assessment for a suspected STD. We already noted that STD clinics are badly known among adolescents (Leenaars, Rombouls, Lammerdin \& Zwetsloot, 1992). Until now, no research was carried out to assess patients' familiarity with STD health services in their immediate environment. Following Lucas and Britt (1963), familiarity concerns either unaided recall (remembrance of a product or service) or aided recall (recognition of a product or service). Likewise, it is advisable for the institutions and providers concerned to know about the expectations of their clients so they can improve the accessibility and quality of STD-services. In order to be market-oriented an analysis of the needs and demands of potential patients is most valuable according to Schweizer (1984). So it is important to find out what STD patients know about and expect from curative STD-services and what service attributes are important to them. This central question may be divided into three research questions:

1. What services and providers in curative STD services are known among STD patients?;

2. What service attributes do STD patients expect from their preferred providers or institutions?;

3. Wich factors explain choice behaviour (choice of STD health services)?;

The Theory of Reasoned Action (Fishbein \& Ajzen, 1975; Ajzen \& Fisbein, 1980) provides a suitable framework for conceptualising choice behaviour (see Figure 6).

It is assumed in the Theory of Reasoned Action that most actions of social relevance are under volitional control and therefore regard an individual's intention to perform an action as the immediate determinant of that action (for example: choice behaviour). This intention, in turn, is seen as a function of two basic determinants: attitude towards the behaviour and the subjective norm. An attitude represents a person's general feeling of (un) favourableness toward performing the behaviour (for example: choice of a particular institution is good). An attitude consists of several relevant beliefs about whether the behaviour results in specific consequences (for example: choice of an STD clinic causes much waiting time) and corresponding evaluations of these consequences (for example: waiting in a waiting room is very annoying). Multiplying the beliefs with the evaluations results in the attitude towards the behaviour. A person's subjective norm is the person's perception that important others think they should perform the behaviour in question (normative beliets) and their motivation to comply with those individuals. According to the theory, attitudes and subjective norms are the sole determinants of intentions. The effects of other variables on intention are assumed to be mediated through the attitude toward the behaviour, the sub- 
jective norm, and/or the relative weights of these components. This model has already been applied successfully to a variety of behaviours (Ajzen \& Fishbeiri, 1980). In this study we operationalised the model to explain choice of an STD clinic, a Family Planning Clinic or a general practitioner.

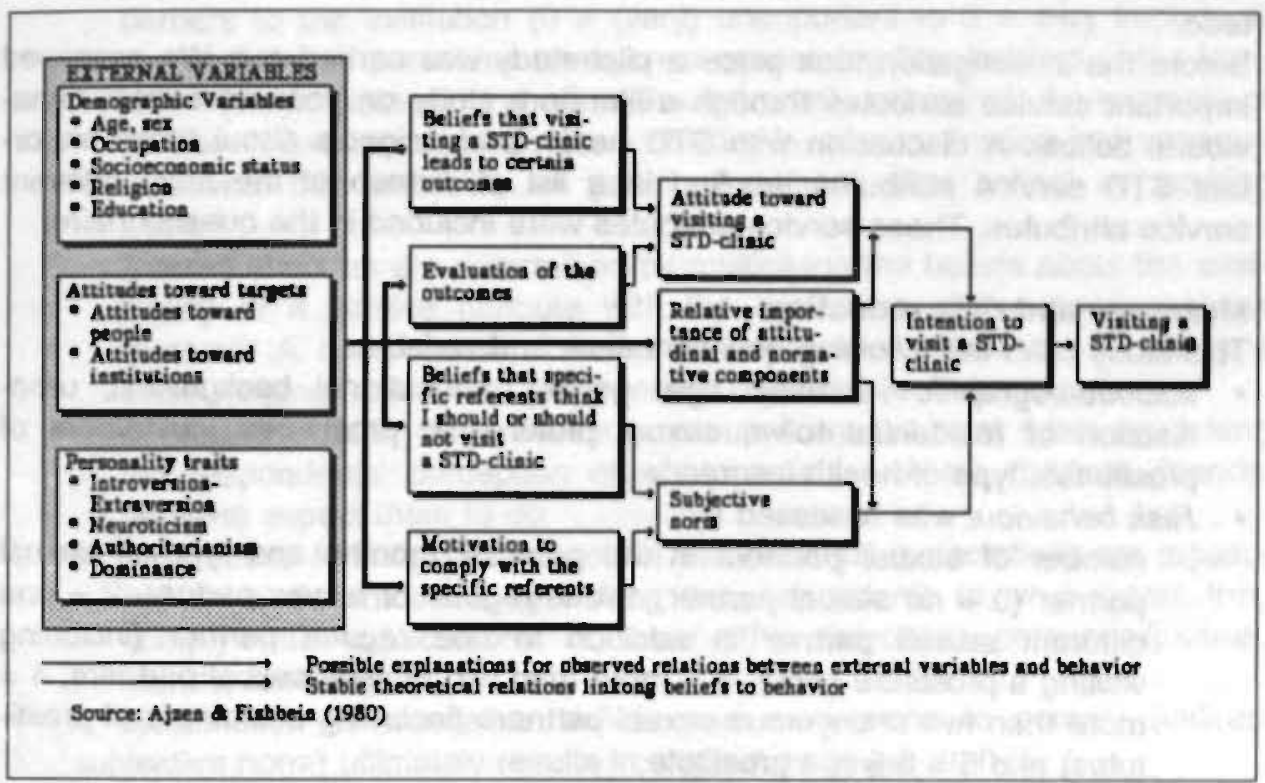

FIGURE 6. THEORY OF REASONED ACTION ADAPTED TO CHOICE OF CIRCUITS

\subsection{Methods}

\section{Procedure and sample}

All subjects $(\mathrm{N}=825)$ visited a health provider for the medical assessment of a suspected (new) STD. Patients visiting health services in eight regions (major cities in the Netherlands) entered the study and in each region participants were recruited from: (1) STD-clinics (low threshold facilities, such as: out-patient STD clinics, institutions with VoMiL regulations and MHSs); (2) Family Planning Clinics; (3) general practitioners. Individuals who are not proficient in Dutch and those who were attending for a check-up of an existing (old) STD and those who were referred to an STD clinic by their general practitioners or other physicians, were excluded from the study. This study lasted about one year in the Family Planning Clinics and among general practitioners, while at the STD clinics and institutions with VoMiL regulation the gathering of respondents took place once or two times a week during nine months. According to the information gathered from the health care providers a response-rate of $90 \%$ was 
obtained from special low-threshold facilities, 95\% from Family Planning Clinics and $96 \%$ from general practitioners.

Health care providers encouraged their patients to take part in the study and gave them instructions in answering the questionnaire. All subjects completed a structured questionnaire after individual consultations. Anomymity was guaranteed.

Before the investigation took place a pilot-study was carried out. We assessed important service attributes through a literature study on possible salient behavioural beliefs. A discussion with STD health professionals about these important STD service attributes resulted in a list of sixteen of the most relevant service attributes. These service attributes were included in the questionnaire.

\section{Measures and date reduction}

This study used the following questionnaires and variables:

- sociodemographic variables: age, gender, educational background, urbanisation of residential town, sexual preference, prostitutes, customers of prostitutes, type of health insurance.

- Risk behaviour was assessed by:

- number of sexual partners in the past six months and type of sexual partner $(0=$ no sexual partner, $1=$ one regular or a new partner, $2=$ one different sexual partner in addition to one regular partner (including visiting a prostitute once), $3=$ more than two familiar sexual partners, $4=$ more than two anonymous sexual partners (including customers of prostitutes) and 5 = being a prostitute;

. frequency of condom use ( $0=$ always, $1=$ irregularly, $2=$ never).

Multiplying number (type) of sexual partners by the frequency of condom-use results in an index for risk behaviour (range: $0=$ no risk behaviour to $10=$ high risk behaviour).

- Previous clinical examination: measured by asking the respondents how many times they visited a physician for a suspected STD in the past two years: never, once, two to four times and four to eight times and more than eight times.

- Unaided recall: spontaneous familiarity with STD heatth services was assessed by asking the respondents from which institutions or health providers they can obtain a medical examination for a suspected STD.

- In accordance with Ajzen and Fishbein's theory (1980) but measured in a slightly different way, attitudes to behaviour and subjective norms were assessed by measuring their proposed constituents, namely:

behavioural beliefs: measured on a 4-point scale $(0=$ disagree entirely to 3 = agree completely) by asking respondents to what extent they expect sixteen service attributes to be available at the institution or with the provider of their choice (i.e. beliets about expected consequences of 
behaviour). One example of an item is: "When I ask this health provider for an STD examination, I expect to have to wait a long time in the waiting room.

outcome evaluations: measured on a 4-point scale in terms of perceived importance concerning consequences of attributes, benefits of and barriers to the institution ( $0=$ (very) unimportant to $3=$ very important) and in terms of perceived annoyance concerning four barriers of the institution ( $0=$ not annoying at all to $3=$ extremely annoying). An example of an item: How important do you consider in an examination into venereal diseases: the possibility of getting treatment after school or working hours?

Indirect attitudes are determined by multiplying the beliefs about the availability of a service attribute with the evaluation of the consequences involved. A combination of belief and evaluation ultimately results in a scale ranging from 0 to 9 (importance) or from 0 to -9 (annoyance);

normative beliefs: three beliefs, measured on a 4-point scale by asking the respondents' perception of what particular others (partner, friends, families) expect them to do;

motivations to comply with each of these others' expectations are measured on a 4-point scale by asking the respondents to what extent they comply with expectations of three other important persons (partner, friends, families).

A combination of normative beliefs and motivations to comply (indirect subjective norm) ultimately results in a scale ranging from 0-9.

\section{Data analysis}

Statistical analyses have been conducted with the statistical software package SPSS-PC 3.1 (Norusis, 1988). Chi-squared analyses have been used to retrieve connections between social demographic characteristics and unaided recall of services. With the intention to find out whether the respondents who chose the various providers differ in expectations from the provision of services, three groups were distinguished: group 1: respondents, who chose an STD clinic (anonymous treatment, free of charge, walk-in consultating hours); group 2: respondents, who chose a Family Planning Clinic (anonymous treatment, not free of charge, evening consultating hours); group 3: respondents, who chose a general practitioner (not anonymous, depending on the type of health insurance, not free of charge). Respondents only evaluated their chosen health care facilities.

Multivariate variance analysis (MANOVA) and Scheffé multiple comparison tests have been used to study whether respondents in these three groups differ in beliefs and evaluations of service attributes. Discriminant analyses were conducted to make a linear combination of the observed variables to explain 
choice of STD health services with the ultimate goal of distinguishing optimally between respondents belonging to the different groups of choice behaviour. For all analyses, a two-tailed significance level of 5 percent was used.

\subsection{Results}

\section{Description of respondents}

A total of 825 persons completed the structured questionnaire. The mean age was 29 years (range 15-61 years) and $59 \%$ were men. The majority of the respondents had a low $(32 \%)$ or middle $(43 \%)$ educational background and almost half of the respondents (48\%) were working-class employees (unskilled and skilled labourers), $22 \%$ were students. Almost $40 \%$ of the respondents lived in a major city (inhabitants $>200,000$ ). The majority (88\%) reported heterosexual relationships, $6 \%$ of the respondents were prostitutes and $11 \%$ were customers of prostitutes.

\section{Demographic characteristics, risk behavlour and choice behavlour}

A total of 627 respondents sought medical assessment at an STD clinic, 90 sought help at Family Planning Clinics and 108 patients visited GPs for a suspected STD.

Gender $\left(x^{2}=18 ; d f=2 ; p<.005\right)$, educational background $\left(x^{2}=16 ; d f=4 ; p<.001\right)$ and type of health insurance company of the respondent $\left(\chi^{2}=17 ;\right.$ of $\left.=4 ; p<.001\right)$ were related to choice behaviour. Respondents at an STD clinic were more likely to be male and lowly educated, while those seeking medical treatment from their GP were more often women and highly educated. Furthermore, respondents seeking medical treatment from their GP were more likely to be covered by the sickness fund than those who sought an examination at STD clinics and Family Planning Clinics, while respondents at Family Planning Clinics were more often compulsory covered by private insurance. No relationship was found between age, sexual preference and choice behaviour.

About 20\% of the respondents $(\mathrm{N}=126)$ showed a score ' 0 ' on the index for risk behaviour, indicating they did not have unprotected sexual intercourse in the preceding six months (no-risk behaviour); $45 \%$ had an index score 1 or 2 , indicating they had (un)protected sexual intercourse with one casual partner or prostitute (low-risk behaviour); $28 \%$ had a index score 4 or 5 and $7 \%$ had a score 8 to 10, which means having had sexual intercourse with more than two anonymous partners, while irregularly or never using condoms (high-risk behaviour) $(M=2.6 ; s d .=2.4)$.

Women were more likely to show low-risk behaviour $(M=2.3)$ and men more often claimed low to high-risk behaviour $(M=3.4)$ ( $F=7.9 ; p<.001)$. Respondents with a low education were more likely to perform low-risk behaviour $(M=2.4)$ and respondents with a high education more often reported low to high-risk beha- 
viour $(\mathrm{M}=3.2) \quad(\mathrm{F}=4.2 ; p<.01)$. No relationship was found between risk behaviouir and choice behaviour.

\section{Famillarity with STD Health Servicee}

The respondents were asked whether they knew, and if so, from what other institutions or providers they can obtain medical treatment for STDs. Almosit $40 \%$ of the respondents at STD clinics, $38 \%$ of those attending Family Planning Clinics and $36 \%$ of those visiting their GPs were not able to spontaneously name another institution for obtaining STD diagnosis and treatment. From the respondents at a STD-clinic who mentioned one, two or three other STD health services $(\mathrm{N}=250)$ almost $70 \%$ mentioned the general practitioner and $54 \%$, mentioned a Family Planning Clinic. From the respondents visiting Family Planning Clinics and who mentioned one, two, or three other STD health services, $69 \%$ mentioned a STD clinic and $75 \%$ the general practitioner. From respondents seeking care at their general practitioners and who mentioned other STD health services, $30 \%$ indicated an STD clinic and $\mathbf{4 7 \%}$ a Family Planning Clinic.

\section{Reasons for not seeklng medical assessment at a General Practltioner}

Respondents were asked why they did not seek medical treatment from a general practitioner for their suspected STD. Almost half of the responderits indicated that embarrassment $(44 \%)$ and perceived unprofessionality $(42 \%)$ were reasons not to visit their GPs.

\section{Bellefs, evaluations of STD Health Services and cholce behavlour}

To understand why respondents sought a medical examination at an STD clinic while others chose a Family Planning Clinic or a general practitioner, it is worth comparing the three groups at the level of behavioural beliets and outcome evaluations. Table 18 shows the mean behavioural beliefs and mean outcome evaluations of the three groups. To test the overall differencel in behavioural beliefs between the groups, Wilk's lambda was computed. This proved to be significant (lambda $=.70$; multivariate $F=9.6 ; p<.001$ ), which indicates that univariate statistics can be used to locate sources of group discrimination.

Table 18 shows that differences in beliefs about several service attributes of the three types of services were found among respondents. The respondents who chose an STD clinic are more strongly convinced than those who consulted their GP, that they: (would) spend much time travelling, have to interrupt their work and will get the proper medication. Also, respondents at an STD clinic are more strongly convinced than those who are with a Family Planning Clinic and those who are with a GP that they need to make an appointment beforehand. Moreover, the respondents who chose an STD clinic are less strongly convinced than those who are with a Family Planning Clinic that they will get personal treatment. Likewise respondents who are with an STD clinic and a Family 
Planning Clinic are more strongly convinced than those with a GP that they will get professional treatment and that the health insurance company will not be informed about the consultation. Respondents who visited an STD clinic and those who sought help with their GP are less strongly convinced than those a Family Planning Clinic that they will receive personal treatment and that treatment is possible outside working hours, while they are more strongly convinced that treatment is free.

TABLE 18. MEAN SCORES OF BELIEFS AND EVALUATIONS FOR THREE CHOICE-BEHAVOUR GROUPS

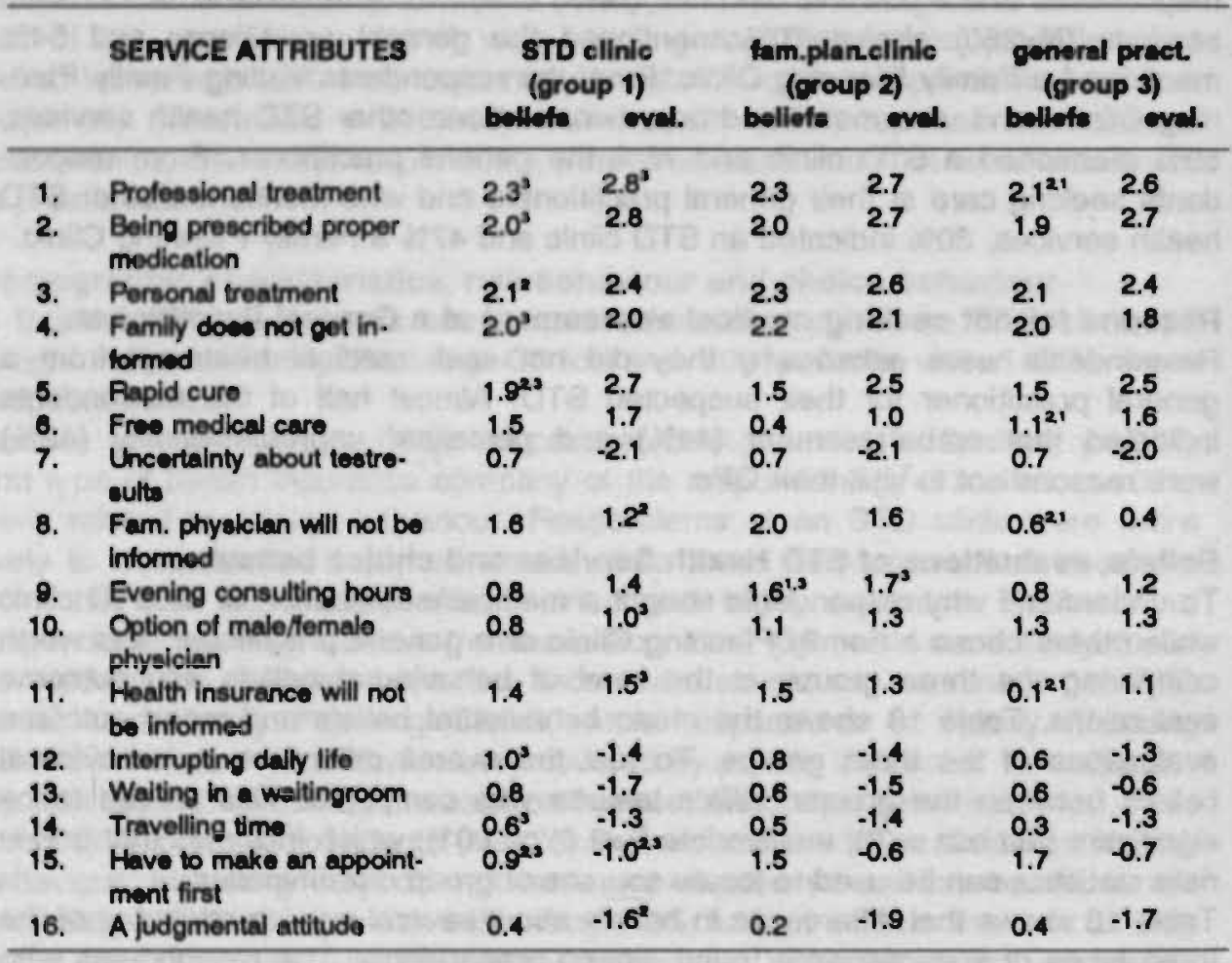

nots: ccore range: Bellots 0 to 3; Evaluations 'importance' 0 to 3; Evaluations 'annoyance' 0 to -3; Subscripts $(1-3)$ Indicate significant differences $(p<.01)$ between pairs of means. The number retere to the different groups.

When we review the evaluation in terms of perceived importance or annoyance of the service attributes, again differences are observed among the respondents with the three types of services. Respondents with an STD clinic consider it more important than those with a GP that they are cured quickly, that the insurance company will not be informed about the STD consultation and that 
they will receive professional treatment, while they find it less important that they can choose between a male or female physician. Likewise, persons at an STD clinic find it more important than those with a Family Planning Clinic that treatment is free, while they find it less annoying if the provider acts derogatorily and find it less important whether or not it is possible to be treated after working hours. Also, respondents who chose an STD clinic find consultations by appointment more annoying than those who chose a Family Planning Clinic or a GP. Finally, respondents who are at an STD clinic find it less important than those with a Family Planning Clinic that they can be treated after working hours.

\section{Social norm and cholce behavlour}

Again a MANOVA was carried out to assess relationships between social pressure from the partner, the families and from friends, and choice of STD health care facilities. Only social pressure from the partner was related to choice behaviour ( $F=4.3 ; p<.01)$. Respondents visiting an STD clinic or a Family Planning Clinic experienced more social pressure trom their partners to seek medical treatment than those seeking medical treatment with their GP.

\section{Determinants of cholce for STD health services}

Before assessing determinants of choice behaviour we carried out a factor analysis on the attitude scales (beliefs * evaluations). This analysis resulted in four factors:

1. Attitude to quality of medical care: four items consisted of attitudes towards getting: professional medical treatment, personal treatment, receive proper medicine and rapid cure. Cronbach's alpha reliability was 0.80 ;

2. Attitude to time investment. measured by four items (travelling time, waiting time, interrupting work and walk-in consultating hours). Cionbach's alpha reliability was 0.74 ;

3. Attitude to anonymous treatment facilities. assessed by four items (that their family doctors, health insurance companies, families and friends do not get informed about the STD consult). Cronbach's alpha reliability was 0.71 .

Because the fourth factor was not an interpretable one (Cronbach's alpha $=0.11$ ) we analysed the rest of the service attributes (free medical treatment, evening consultatiing hours, spend a lot of time commuting, stay uncertain of testresults for a long time, anticipate a professional judgmental attitude) separately.

To better understand the importance of service attributes and social pressure in the choice of a special STD clinic, a Family Planning Clinic or a general practitioner, we compared respondents at STD clinics (group 1) and respondents at Family Planning Clinics (group 2) and those visiting their GPs (group 3). 
TABLE 19. DISCFIMINANT FUNCTION COEFFICIENTS

\begin{tabular}{|c|c|c|c|c|}
\hline \multirow{2}{*}{$\begin{array}{l}\text { cervice } \\
\text { attributes }\end{array}$} & \multicolumn{2}{|c|}{ FUNCTION 1} & \multicolumn{2}{|c|}{ FUNCTION 2} \\
\hline & $\begin{array}{l}\text { stand.diser. } \\
\text { function coeff. }\end{array}$ & $\begin{array}{l}\text { correl. } \\
\text { with funct. }\end{array}$ & $\begin{array}{l}\text { stand. discr. } \\
\text { hunction coeff. }\end{array}$ & $\begin{array}{l}\text { corrol. } \\
\text { with funce. }\end{array}$ \\
\hline Evening consulting hours & -.79 & .61 & .24 & .12 \\
\hline Free medical care & 70 & .59 & .00 & .33 \\
\hline Option of malo/femalo phyeician & .39 & .11 & .70 & .29 \\
\hline Time-investment &.$\infty$ & .11 & -.48 &. .43 \\
\hline Anonymons treatment & .03 & .22 &. .38 & .73 \\
\hline Partner pressure & -.07 & .03 & .28 & .32 \\
\hline Canonical correlation & .40 & & .30 & \\
\hline Wilk'e lamba & $.76^{\circ}$ & & $.91 *$ & \\
\hline
\end{tabular}

Discriminant analysis revealed two functions, including six variables. As revealed by the evaluation of group centroids, the first function most clearly differentiates between respondents who attended a Family Planning Clinic (groupcentroids $=1.2$ ) and those who attended an STD clinic (groupcentroids $=-14$ ) or their GPs (groupcentroids =-.19). The function derived is largely defined by the attitude towards evening consultating hours as well as the attitude to free medical care. Compared to respondents visiting an STD clinic or their GPS, those attending a Family Planning Clinic had a more positive attitude to evening consultating hours and a more negative attitude to free medical care offered by the institution of their choice.

The group centroids indicated that the second function most clearly differentiates between respondents who attended their GPs (groupcentroids $=-.78$ ) and those who visited an STD clinic (groupcentroids $=.14$ ) or a Family Planning Clinic (groupcentroids $=-.08$ ). This function is primarily defined by the attitude to the option of a male/female physician, the attitude to time investinent and the attitude to anonymous treatment possibilities. Compared to those visiting their GPs, respondents who attended an STD clinic or a Family Planning Clinic had a more positive attitude towards the option of a male/female physician, a more negative attitude to time-investment and a more positive attitude to anonymous treatment possibilities and they experienced more pressure from their partner. The total variance in discriminant scores explained by differences in attitudes and social pressure between the groups was small, namely $16 \%$ for the first function and $9 \%$ for the second function.

Classification of choice behaviour based on these six variables led to $55 \%$ correctly classified cases. Half of the respondents at the STD clinics were correctly classified as well as two-thirds of the respondents visiting a Family Planning Clinic and $70 \%$ of the respondents who visited their GPs. 
Repeaters and loyallty

Half of the respondents claimed they never had a medical examination for STDs before, one-third had visited a physician for an STD once before and onequarter had STD check-ups at least twice in the past two years.

A strong relationship existed between past choice of STD health services and present choice of STD health services (eta $=.62 ; \mathrm{df}=4 ; \mathrm{p}<\mathrm{.0001}$ ). Almost all respondents (95\%) who chose an STD clinic in the past, attended an STD clinic again. In addition, almost three-quarters of the respondents who chose a Family Planning Clinic in the past, sought medical treatment at the Family Planning Clinic again. On the contrary, half of the respondents who obtained medical treament from their GPs in the past, visited an STD clinic this time. Most important reasons mentioned for this shift in health providers were: perceived unprofessionality (64\%), embarrassment $(45 \%)$ or time investment $(21 \%)$.

Most respondents stated that in the case of future STDs they will also get medical treatment at the same institution or health care provider of their first/second choice.

\subsection{Discussion}

The development of new STD Health Services as well as the success of existing programmes are important tools for STD managernent and STD control strategies.

The purpose of this study was to try to identify those service attributes which would improve patient satisfaction with the service in order to encourage them and others to attend. Several service attributes in the quality of medical care, costs and barriers, clinical staff, location and clinic hours were discussed. The generalisability of the results is, however, limited by selection procedures. The persons who are not proficient in Dutch (mainly ethnic minorities) have been exluded from participation while other STD clinic attenders may be well represented. Yet, it is unknown how many individuals seek a medical exarnination for a suspected STD at STD clinics, Family Planning Clinics and General Practitioners in both urban and rural regions in the Netherlands.

Additionally, it is possible that the GPs who participated in this study are more tolerant towards STDS and STD patients. This could mean a positive bias of the expectations of the services with GPs of persons who visited their GPs for medical treatment. By having the providers encourage participation we may have also introduced a bias which may have affected not only the response ralte in a positive sense, but also the findings reported here. It is also important to note that in this study determinants of actual (past) behaviour rather than intentions to choice behaviour, were investigated with psychosocial variables measured after the behaviour has occured. 
It appears that other institutions for STD health care than the one chosen, especially out-patient STD clinics, are not well known among a relatively large number of patients. It is therefore advisable to pay much more attention to the existence of special STD clinics, especially in mass media campaigns.

After questioning patients' views of services, health care providers are able to plan new developments which are most suited to local needs and demands. In addition, the results of this study may be useful in making persuasive arguments in favour of speciall low-threshold treatment facilities, especially when competing for limited resources. As expected, most patients evaluate aspects of the quality of medical care as very important attributes. It is advisable to work with physician-designed protocols in order to quarantee quality of medical care at different institutions. When considering the image and evaluation of STD health services, it is striking that service attributes which concern the taboo on STDs, such as the possibility of anonymous treatment, are still important in STD health care. This means that one should consider this desired anonimity when developing new STD health services. An attempt can be made to avoid contact between STD patients when designing the waiting room and organising operating hours. Most patients visiting an STD clinic did not want their GPs to be informed about the STD examination afterwards as well as to receive the results of the STD examination. The general practitioner should therefore not be informed of the clinical attendance of his patients. Moreover, it should be pointed out that specialists as well as general practioners have professional secrecy and that the STD request will therefore be treated with confidentiallity not only by specialists at STD clinics but also by GPs.

The differences in beliefs and evaluations between the patients at the different instltutions show that STD clinics distinguish themselves from GPs and/or Family Planning Clinic in the following service attributes: professional treatment, proper medication, GP will not be notified of the STD consultation, the insurance company will not be notified of the STD consultation, free treatment, walk-in consultations and rapid cure. Most of the time, respondents who attend a STDclinic attach more importance to these attributes than the two other groups of respondents. From these findings we conclude that the raison d'etre of low threshold STD out-patient departments is confirmed as institutions where one can obtain free, anonymous and professional care.

On the other hand, STD clinics distinguish themselves negatively from Family Planning Clinics and/or GPs concerning: travelling time, interrupting work, waiting time, personal treatment and possibility of treatment after working hours. With the exception of personal treatment these service attributes deal with a perceived long investment of time for medical care and evening consultating hours. Despite the variable and unpredictable volume of patients inherent in walk-in type clinics, waiting time can be greatly reduced by maintaining an efficient flow of patients from the time they sign in until they are discharged. 
Franklin and Stolz (1984) gave excellent examples of ways through which long and decompensating delays are anticipated and avoided.

Considering the service attributes where the Family Planning Clinics in particular obtain a high score, it speaks for itself that STD clinics should take into consideration, with the selection of personnel, such attributes as: personal approach and a tolerant attitude towards STD patients. Moreover, it is advisable to hire both male and female physicians and males and females in nursing staft.

Of the determinants for choice of health services, none was more important than the existence of evening or weekend consultating hours. Therefore, operaling hours should take into account the way of life of patients and daily variations in the demand of services. In a recent study on genitourinary medicine services (STD clinics), Munday (1990) also concluded that it is no longer sufficient to provide a competent service, but that STD clinics should also offer services in an environment conducive to the consultations at a time suitable for the patient. However, these service attributes accounted for only $24 \%$ of the variance in attitudes to choice behaviour between the three groups. This means that other factors (have to) play a role in choice behaviour as well.

That previous behaviour predicts subsequent behaviour is only partly ascribed through the results. The relatively important shift of patients from GPs to STD clinics was mainly explained by perceptions of unprofessionality of GPs and embarrassment among clients. In addition, unprofessionality and embarrassment were also reasons for the other patients not to visit their GPs. Perceived unprofessionality of GPs indicates that STD clinic attenders do not evaluate their GPS as specialists in the medical treatment of STDs. Physician designed-protocols adjusted to the practice of general practitioners may also improve this perceived professionality of GPs.

Clearly, this study did not examine all possible determinants of choice behaviour. Issues of perceived behavioural control (the perceived ease or difficulty of performing the behaviour (Ajzen, 1987)) and self-efficacy expectations (Bandura, 1989) were not included in this study. Most theories of consumer behaviour (Howard \& Sheth, 1969) also consider exogenous factors (such as importance of the purchase, personality traits, routinised response behaviour, limited and extensive problem solving), perceptual constructs (such as: ovent search, stimulus ambiguity, attention) and confidentiality. The resulting satisfaction or dissatisfaction is assumed to feed back into the system.

An important methodological issue is that, because of the length of the questionnaire, we only measured attitudes to the choice of one institution or health providers, namely their chosen health care provider. The results showed that the three groups differed mostly in attitude towards professional treatment, receiving proper medication etc. However, these differences fade because of the reported high absolute scores on these attitudes. This means that the difference between the groups is not the relative importance of this beliet, but 
the fact that the group that chose an STD clinic feels they receive (for example) professional treatment from the STD clinics and those who went to their GPs also feel they receive professional treatment from them. We do not have any data about how patients at STD clinics rate the professionalism of their GPs. Assessing differential beliefs and attitudes may be more appropriate in a choice situation (Aizen \& Fishbein, 1980) than are the separate measures of these variables with respect to each institution. 


\section{CONSUMER BEHAVIOUR OF ADOLESCENTS WHEN SUSPECTING A} SEXUALLY TRANSMITTED DISEASE

Published as: Leenaars, P.E.M., Rombouts, R., Lammerdin, A.G., Zwetsloot, L.M. (1992). Consumentengedrag van middelbare scholieren bij vermoeden van een Seksueel Overdraagbare Aandoening. Tijdschrift voor de Sociale Gezondheidszorg, 70(2), 95-102.

\subsection{Introduction}

Several studies into the sexual behaviour of adolescents show that approximately one-third of the pupils aged 14 to 17 have had sexual intercourse (Gieling \& Heuzer 1988; Delil 1988; De Wit, Kok, Timmermans \& Wijnsma, 1990). Moreover, a recent study Vogels and Van der Vliet (1990) showed that half of 11,000 adolescents aged $\mathbf{1 7 . 5}$ have had sexual intercourse. Using condoms is one of the most effective methods of preventing Sexually Transmitted Diseases (STDs). However, approximately half of the pupils who indicated to have had sexual intercourse do not always use them (Nogels \& Van der Vliet 1990). These findings are confirmed by Leenaars, Rombouts, Lammerdin \& Zwetsloot (1991). How often adolescents contract an STD cannot exactly be found out due to underreporting. On account of the national reporting figures of gonorrhea it was estimated (Van de Laar, van den Hoek, Pickering, van Griensven, Coutinho \& van de Water, 1990) that approximately 3 out of 1,000 females aged 15 and 2 out of 1,000 males aged 15 contracted gonorrhea in the Netherlands in 1987. The incidence rate of gonorrhea among adolescents increases with age up to approximately five out of one thousand adolescents aged 20. De Vries (1988) also suspects that about 20,000 addescents between the ages of 15 and 25 annually contract STDs in the Netherlands, such as: gonorrhea, syphilis, Chlamydia trachomatis, Herpes simplex and condylomata (reference year, reporting figures 1985).

Because of the increase in difficult or often untreatable viral infections '(e.g. Chlamydia trachomatis, Herpes Simplex, Human Papilloma Virus and HIV-infection) among the Dutch population, primary prevention is focused on these 'second generation STDs'. However, early detection of STDs also deserves high priority. The taboo on STDs and the access to curative STD-services can, however, play an impeding role in activities focused on early detection of STDs.

The structure, organisation, and funding of curative STD-health care in the Netherlands is quite complicated. This is also enhanced by regional differences in the provision of assistance (Leenaars, Oliehoek \& Schrijvers, 1992). Generally speaking, a distinction can be made between (1) the regular circuit, to which the general practitioner and facilities with low-threshold regulations belong (STD 
clinics and Municipal Health Services) and, (2) the non-regular or alternative circuit including the Family Planning Clinics.

The regular circult

The formal state of affairs is that persons who suspect an STD go to see a General Practitioner (GP) for medical assessment. Diagnosis and treatment of an STD can take place with the GP concerned or one can be referred to an independent specialist or a specialist connected to a hospital. Funding of consultation, diagnosis and treatment takes place through legal medical insurance.

Since 1976, the department of Welfare, Public Health and Culture has subsidised out-patient STD clinics in the four largest cities (Amsterdam, Rotterdam, The Hague and Utrecht) which have free access and provide anonymous medical care to people who: (1) are not insured, (2) do not have a GP or (3) wish to remain anonymous because of the taboo on STDs. In order to make these low-threshold facilities possible in the remaining towns, the VoMil. regulation' was set up (named after the then depantrnent of Public Health and Environmental Affairs). Persons who do not want to visit a GP or low-threshold facilities, can, by virtue of this regulation, be referred to an independent dermatologist or a dermatologist in a general hospital by the department for noncurative STD-health care of Municipal Health Services (MHSs). At the moment, the costs of diagnosis and treatment of all STDs for residents in the Netherlands (the inhabitants) who use these low-threshold facilities, are paid for by the AWBZ-foundation. The costs of diagnostics and treatment of all STDs for nonresidents are still paid for by the State. Apart from these out-patient STD clinics and institutions with VoMil-regulations, MHSs can, under certain conditions, work with curative venereal disease prevention since 1985. Until recently, nine MHSs have been recognised as such in the Netherlands. This means that diagnosis of classic STDs in these institutions is financed by the state. The costs of consultations and treatment, however, are in most cases paid for by the STD patients themselves (own contribution) or by the institution.

The non-regular or alternatlve clrcult

Apart from the regular circuit, there are a number of Family Planning Clinics in the Netherlands that deal with STD prevention and curation. Since 1986, persons who suspect an STD can go here without referral, but usually have to pay for the costs of consultations, diagnosis and treatment themselves. A number of Family Planning Clinics also refer persons who have difficulties paying for consultations, to a low-threshold facility.

As emerges in the above description, the structure of curative STD-health care is quite vague. To what extent this complicated structure has repercussions on persons who seek medical assessment is unknown. 
It is important for curative STD-health care that potential STD patients are familiar with the provision of assistance in the immediate environment. Following Lucas and Britt (1963), it concerns spontaneous familiarity (recall of a product or service) or assisted familiarity (recognition of a product or service). Recent research, among 825 persons who have already sought medical assessment for an STD, shows that approximately half cannot 'spontaneously' name other institutions for curative STD-health care (Leenaars, Rombouts \& Schrijvers, 1990). To what extent these results apply to the group of adolescents is as yet unknown.

Likewise, it is advisable for the institutions and providers concerned to know the expectations of their clients in order to improve the access and quality of STD. services. In order to be market-oriented an analysis of needs and demands of potential patients is, according to Schweizer (1984), most important. From this approach seen it is important to find out what adolescents now know ancl expect from curative STD-health care in their immediate environment and which service attributes are important to them. This central question may be divided into three research questions:

1. which services and providers of curative STD-health care are familiar among adolescents?;

2. which providers or services will the adolescents turn to seeking medical assessment when suspecting an STD?;

3. which service attributes do adolescents expect from their chosen providers or services and which service attributes play a role in the intention of choice of circuits?

In order to answer the third question, concepts from Fishbein and Ajzen's theory (1975) were used. Ajzen and Fishbein (1980) assume that behaviour (in this case circuit choice) can be predicted from the intention to perform the recommended behaviour. The intention to behaviour is, according to their theory, a function of two determinants: the attitude towards the behaviour concerned and the subjective norm. The attitude may, in turn, be explained by beliefs about the expected consequences of the behaviour (beliets) and the evaluation of these consequences (evaluations). According to their model, the subjective norm may be explained by the beliefs a person has about the opinions of others to show the behaviour on the one hand and by the motivation to comply to the opinion of others on the other. Yet, Kok, Buisman, Kampschuur, Krist and Van der Vange (1981) state that the influence of the social environment depends on the, public or not, showing of the behaviour. In this study, the behaviour under study is a behaviour in which anonimity may play a major role. We have therefore assumed that it concerns the displaying of non-public behaviour and suspect that the social environment only plays a role through the existence of specific altributes, such as the possibility of anonymous treatment. 
In the town of the Hague, where the research took place, the provision of STD assistance consists of two out-patient STD clinics for the curation of venereal diseases. In addition, one can seek medical care at Family Planning Clinics. Moreover, GP8, independent dermatologists and gyneacologists are present who practice STD curation. Drug-addicted prostitutes can also seek medical care al a 'living room project'.

\subsection{Methods}

\section{Procedure and sample}

In order to answer the questions, a survey was distributed among adolescents in The Hague, mid-February of 1988 . Two schools for intermediate vocational education, three schools for part-time education and the highest grades of four school communities participated in this study. For the benefit of an even distribution over the various school years, several classes were visited at school. Under the direction of the researchers, the questionnaires were completed classically and anonymously and then collected in a sealed envelope. Three hundred and eight pupils were approached to participate in this study. Two pupils refused to cooperate when examining the contents of the questionnaires, and, after opening the envelopes, another 36 respondents were excluded because more than 7 answers were missing.

The questionnaire was more or less completed by 270 pupils, 130 men and 140 women. The mean age of these respondents was 18 years with as extreme limits 15 and 22. The division per type of school was as follows: part-time education $14 \%$, intermediate vocational education $24 \%$, secondary modern school $31 \%$, grammar school $31 \%$. Over half of the pupils is of Dutch descent (79\%), one-fith is of Surinam descent and the remaining pupils are of Turkish, Moroccan or Antilian descent $(10 \%)$. Half of the pupils indicated that they are non-religious and the remaining respondents are Catholic (13\%), ProtestantChristian (15\%) or Islamic/Hindu (21\%).

In this study, persons who have had sexual intercourse belong to the sexuallyactive when contact has taken place between the genitals or mouth-anal genitals. Of the 270 respondents, almost half has not been sexually active during the past year, while $35 \%$ has had sexual intercourse with one partner, $6 \%$ with two partners and $13 \%$ has had sexual intercourse with more than two partners. Of the entire study population $5 \%$ were tested for an STD in the last two years and $2 \%$ twice or more.

\section{Measures}

In this study, the following variables were measured:

- Social demographical characteristics: age, gender, education, cultural background, religion; 
- Sexual activities are measured by asking the respondents the number of persons they have had sexual intercourse with in the past twelve months. What is meant by sexual intercourse is indicated in a separate commentary: necking plus contact between genitals and/or mouth genitals (alternatively including anal contact);

- Assisted familiarity with services of curative STD-health care is measured in imitation of Lucas and Britt (1963) by asking the respondents which services, of a number of described services and providers, treat venereal diseases: out-patient STD clinics, Municipal Health Services, Family Planning Clinics, specialists in hospitals, out-patient clinics for homosexual men, specialists independent of hospitals and GP. Because of the method used (yes-or-no questions and marking the institutions involved) the recognition of institutions for curative STD-health care (i.e. assisted familiarity) is important;

- The image of institutions for curative STD-health care is measured by asking respondents to what extent they expect sixteen service attributes to be available (such as: free treatment, walk-in consultations, professional treatment, possibility of anonymous treatment etc.) at the institution or with the provider of their choice (i.e. beliefs about expected consequences of circuit choice). One example of an item is: When I go to the provider of my choice for treatment, I expect that I will have to wait a long time in the waiting room. On a 4-point scale ( 1 = disagree entirely to $3=$ agree entirely) is indicated to what extent they expect to find the service attributes concerned;

- Evaluation of the service attributes is measured in terms of perceived importance concerning the benefits of the institution and in terms of perceived annoyance concerning the barriers of the institution. An example of an item: How important do you find for examination of venereal diseases: - to be treated after school or working hours? It is indicated on a 4-point scale to what extent the service attributes are considered important $(0=$ (very) unimportant to $3=$ very important). How annoying do you find for exarninalion of venereal diseases: - to spend much time commuting? It is indicated on a 4-point scale to what extent one finds the feature concerned annoying $(0=$ not annoying at all to $-3=$ extremely annoying);

- Aspects of attitude are determined by multiplying the belief about the availability of a service attribute with the evaluation of the attribute involved. A combination of belief and evaluation ultimately results in a scale ranging from 0 to 9 (importance) or from 0 to -9 (annoyance);

- The intention to circuit choice is measured by asking the respondents through an open question what institution or provider they will choose when they suspect an STD. 


\section{Date analyeos}

Statistical analyses were conducted with the statistical softwarepackage SPSSPC 3.1 (Norusis, 1987). With the intention of finding out if the respondents who will choose the various providers or facilities differ in expectations from the provision of services, several analyses were carried out. Three groups have been put together on the basis of intention to circuit choice:

group 1: respondents, who choose the GP;

group 2: respondents, who choose the Family Planning Clinic;

group 3: respondents, who choose a specialist or specialist institution.

Because of the srnall number of respondents who intend to go to an STD clinic, MHSs, or other specialist institution/provider, the third group was put together from respondents who intend to go to MHSs, a specialist in or outside a hospital, or an STD clinic.

Chi-squared analyses were used to retrieve the connection between social demographic characteristics and familiarity with services.

Through analyses of variance (and Scheffe-tests) it was observed if respondents who intend to choose a 'specialist', Farnily Planning Clinic or a GP differ in expectations about service attributes. Multivariate analysis of variance (MANOVA) has been used to study whether the intention to choice of circuits can be explained through the attitude towards the various service attributes. The statistic significance level has continually been set at $5 \%$.

\subsection{Results}

Famillarlty with curatlve STD-health care

A total of $83 \%$ of the respondents recognises (assisted familiarity) the GP as a treater of venereal diseases, three-quarters recognises the Family Planning Clinic, $40 \%$ the MHSs and one-third the specialist in a hospital. Only $8 \%$ of this group of adolescents recognises the STD clinic and the independent specialist as the institutions or providers for curative STD-health care. In order to assess how many institutions and providers the pupils actually know about, a sumscore was calculated for the number of recognised institutions and providers. On average, the pupils know two to three institutions/providers where one can seek treatment of STDs. A relationship exists between the number of recognised facilities and cultural background $(F=9.1 ; p<.000)$. Pupils of Dutch cultural background know an average of two to three institutions or providers, whereas pupils of Surinam background know an average of one to two institutions. Gender, age and education do not show a relationship with the number of recognized institutions. 


\section{Intention to cholce of circults}

If these adclescents suspect an STD, almost $43 \%$ intend to go to the GP. $26 \%$ to the Family Planning Clinic, $17 \%$ to a specialist in the hospital, $5 \%$ to the MHSs, $3 \%$ to an STD clinic, $2 \%$ to an independent specialist and the rernaining respondents $(4 \%)$ intend to choose an other institution. A relationship exists between gender and intention to choice of circuits $\left(\Psi^{2}=8.7 ; p<.01\right)$. Fernales: seeking medical assessment of a suspected STD are more likely than males to intend to go to a Family Planning Clinic and males intend to go see their GP more often than females. Moreover, pupilis who intend to go to their GP are ${ }_{4}$ on average, older than those who will choose the Family Planning Clinic, or those who are thinking of going to 'a specialist' $(F=4.2 ; p<0.05)$. No relationship exists between education and cultural background on the one hand and intention 10 choice of circuits on the other.

\section{Image of Institutione for curative STD-health care and intention to cholce of clrcults}

More than half of the respondents more or less expect (aimbivalent to strongly agree) with a medical exarnination: to have to wait a long time in the waiting room, to have to interrupt work, to make an appointment beforehand, to be treated after school or work, to receive free treatment, to receive proper medication, to stay uncertain about the results, that family, friends and the medical insurance company will not be informed about the STD examination, that they can choose between a male and female physician and that they will be cured rapidly. In addition, nearly half of these pupils more or less expect (ambivalent to strongly agree) that they may be critised for their sexual behaviour, their GP will be informed about the STD examination, and that they will spend a lot of time commuting.

Through analysis of variance it has been investigated whether the respondents, who intend to see a specialist, a GP or a Family Planning Clinic respectively, differ in beliefs about the provision of care by the institution of their choice (see Table 20).

It appears from Table 20 that persons who intend to go to a Family Planning Clinic differ in beliets to those who intend to go to a 'specialist' or a GP. Pupils who will go to a Family Planning Clinic expect more strongly than the two other groups, that family, friends and their medical insurance company will not find out about the STD examination and that they have the option of a male or female physician. Moreover, they do not expect as strongly than the two other groups that they need to make an appointment beforehand. They also expect more strongly than those who choose a 'specialist' that their GP will not be informed about the STD examination. Of course, persons who intend to go to their GP expect less strongly than the two other groups that their GP will not be informed about the STD examination. Table 20 also shows that persons who will go to 
their GP expect less strongly than the two other groups that they will spend a lot of time travelling and that work or school will be interrupted for a long period of time. Finally, Table 20 shows that pupils who will go to a 'specialist' expect more strongly to have the option of a male or female physician than those who intend to go to their GP.

TABLE 20. MEAN 8COFES FOF BELIEFS AND EVALUATIONS OF SERMCE ATTRIBUTES BY INTENTION TO CHOICE OF CIACUTS

\begin{tabular}{|c|c|c|c|c|c|c|}
\hline cervice attribuibe & \multicolumn{2}{|c|}{$\begin{array}{l}\text { "epeclallot' } \\
\text { bollote ovel. }\end{array}$} & \multicolumn{2}{|c|}{ general prect. } & \multicolumn{2}{|c|}{ fam. plann. clinke } \\
\hline Evening consulting hours & 1.5 & 1.5 & 1.5 & 1.6 & 1.7 & 1.6 \\
\hline Free medical care & 1.1 & $1.7 *$ & 0.9 & $1.3^{*}$ & 1.1 & $1.8^{\star}$ \\
\hline Family does not get. informed & $1.4^{m-m}$ & 1.3 & $1.5^{\text {to }}$ & 1.4 & $2.1=$ & 1.7 \\
\hline Being prescribed proper medication & 2.3 & $2.8^{\circ}$ & 2.2 & $2.6^{*}$ & 2.2 & $2.8^{*}$ \\
\hline Quick cure & 1.8 & 2.7 & 1.7 & 2.6 & 1.8 & 2.8 \\
\hline Professional treatment & 2.4 & $2.7 \%$ & 2.3 & $2.7^{*}$ & 2.3 & $2.9^{*}$ \\
\hline Poreonal treatment & 2.0 & $2.2^{\star}$ & 2.2 & $2.1^{*}$ & 2.2 & $2.5^{\star}$ \\
\hline GP will not be informed & $0.7=$ & $0.6^{*}$ & $0.2^{\text {ntw }}$ & $0.4^{*}$ & $1.5^{\star \Delta}$ & $g^{*}$ \\
\hline Option of male/lemale physician & $1.3^{\text {nt }}$ & 1.4 & $0.9^{\text {m*t }}$ & $1.1^{*}$ & $1.6^{* *}$ & $1.7^{*}$ \\
\hline Health insurance will not be informed & $0.8^{\text {*tat }}$ & $0.8^{*}$ & $0.8^{\text {tat }}$ & $0.7 *$ & $1.2^{* *}$ & $1.1^{*}$ \\
\hline Wailting In a walting room longly & 1.0 & -1.5 & 0.7 & -1.3 & 0.8 & -1.4 \\
\hline Have to make an appointment first & $1.8^{\text {the }}$ & -0.7 & $1.7^{m+}$ & -0.5 & $1.5^{\text {t*t }}$ & -0.5 \\
\hline Internupting daily life & $1.0^{\text {mat }}$ & -1.4 & $0.5^{\text {st* }}$ & -1.6 & $0.8^{\text {m* }}$ & -1.3 \\
\hline Uncertainty about testresults & 0.7 & -2.5 & 0.9 & -2.6 & 0.7 & -2.6 \\
\hline Time spend travelling & $0.5^{\text {th }}$ & -1.4 & $0.2^{*}$ & -1.3 & $0.4^{\text {th }}$ & -1.1 \\
\hline A judgmental attitude & 0.7 & -2.1 & 0.7 & -2.0 & 0.7 & -2.1 \\
\hline
\end{tabular}

note: $p<0.05$ : difference in evaluations; $p<0.05$ : difference in beliefs

Evaluation of service attributes and Intention to cholce of clrcults

More than half of the respondents find it important to very important that: they can be treated aner (school)hours, get tree treatment, family and friends are not informed about their STD examination, they get proper medication, they are cured rapidly, they get professional and personal treatment and that they can choose a male or female physician. Apart from this, it has been established that a little more than half of the respondents find it unimportant to very unimportant that: their STD examination stays unknown to their GP and medical insurance company. Likewise, it became apparent that more than half of the respondents find it (extremely) annoying that: they have to wait a long time in the waiting room, they have to interrupt work for a long period of time, they stay uncertain 
of the results for a long time, they spend a lo of time travelling, and that they find criticism about their sexual behaviour annoying. However, $56 \%$ of the respondents find having to make an appointment beforehand not bothersome. Analysis of variance has been conducted to investigate whether pupils who intend to go to a 'specialist', GP or Family Planning Clinic differ from each other when evaluating the service attributes.

Table 20 shows that pupils who intend to go the Family Planning Clinic differ in evaluation of service attributes from pupils who will choose a 'specialist' or GP. Pupils who intend to go to a Family Planning Clinic find the following attributes more important than the two other groups: (1) personal treatment; (2) professional treatment; (3) the GP is not informed of the STD examination; (4) the oplion of a male or female physician and (5) the medical insurance company is not informed of the STD examination. Moreover, Table 20 shows that the respondents who go to a GP find tree treatment and proper medication nol as important as those who intend to choose the Family Planning Clinic or a 'specialisf'.

Also, students who intend to go see a 'specialist' find it a little more important than those who intend go to their GP, that the GP is not informed about the STD examination and that they have the option to choose a male or female physician.

Attitude towards eervice attributes and the Intentlon to cholce of clrcults

In order to examine whether the intention to choice of circuits can be explained by a difference in attitude aspects (beliefs $x$ evaluations) between those who intend to see a 'specialist', GP or Family Planning Clinic, Multivariate Analysis of Variance was conducted on the scores compiled.

This analysis shows that pupils who suspect an STD intend to choose a 'specialist', GP or Family Planning Clinic differ in a combination of aftitude aspects (Hotellings' $T^{2}=.63 ; p<.000$ ). The results of the univariate tests show which attributes are responsible for the observed differences between pupils who intend to choose a 'specialist', GP and Family Planning Clinic respectively.

Table 21 shows that pupils who intend to go to the Family Planning Clinic, differ from the other two groups concerning four attitude aspects: (1) Family and friends do not get informed of STD examination, (2) the GP is not informed of the STD examination, (3) the medical insurance company is not informed of the STD examination, and lastly (4) the option of a male or female physician. Table 21 also shows that pupils who intend to go to a 'specialist' have a lower score on the attitude aspect 'a possibility of personal treatment' than the two other groups. Moreover, the pupils who will go to a specialist score higher on the attitude aspect 'option of a male or female physician' than those who will go to a GP. 


\section{TABLE, 21. MEAN SCORES AND F-VALUES FOR BELJEFS AMD EVALUATIONS BY MTENTION TO CHOICE OF CIRCUITS}

\begin{tabular}{|c|c|c|c|c|c|}
\hline \multirow[b]{2}{*}{ service attributea } & \multirow{2}{*}{$\begin{array}{l}\text { 'epecialiot' } \\
\text { bellefe } x \text { oval. }\end{array}$} & \multirow[t]{2}{*}{$\mathbf{G P}$} & \multirow{2}{*}{$\begin{array}{l}\text { Fam. Plan. Clinic } \\
\text { bellefe } x \text { oval. }\end{array}$} & \multirow[b]{2}{*}{ f-valus } & \multirow[b]{2}{*}{ aign. } \\
\hline & & & & & \\
\hline $\begin{array}{l}\text { Evening consulting } \\
\text { hours }\end{array}$ & 2.3 & 2.5 & 2.8 & 0.85 & 0.42 \\
\hline Free medical cere & 2.1 & 1.4 & 2.2 & 2.80 & 0.08 \\
\hline $\begin{array}{l}\text { Family does not get } \\
\text { informed }\end{array}$ & 2.5 & 2.5 & 40 & 7.32 & 0.00 \\
\hline $\begin{array}{l}\text { Being prescribed } \\
\text { proper' medication }\end{array}$ & 6.7 & 6.1 & 6.3 & 1.18 & 0.30 \\
\hline Rapid cl & 5.1 & 4.7 & 5.3 & 0.79 & 0.45 \\
\hline $\begin{array}{l}\text { Professional treat- } \\
\text { ment }\end{array}$ & 6.7 & 6.6 & 7.0 & 0.50 & 0.60 \\
\hline Personal treatment & 5.5 & 6.5 & 6.5 & 3.00 & 0.05 \\
\hline $\begin{array}{l}\text { QP' do not get infor- } \\
\text { med }\end{array}$ & 0.6 & 0.0 & 1.7 & 34.27 & 0.00 \\
\hline $\begin{array}{l}\text { Option of malefiema- } \\
\text { lo phyolician }\end{array}$ & 2.6 & 1.2 & 3.3 & 12.62 & 0.00 \\
\hline $\begin{array}{l}\text { Health insurance } \\
\text { does not got infor. } \\
\text { med }\end{array}$ & 1.0 & 0.8 & 1.5 & 3.75 & 0.02 \\
\hline $\begin{array}{l}\text { Waiting in a waiting } \\
\text { room for a long tirne }\end{array}$ & -1.6 & -1.2 & 25 & 1.32 & 0.12 \\
\hline $\begin{array}{l}\text { Have to make an ap- } \\
\text { point-ment first }\end{array}$ & -1.2 & -0.8 & -0.7 & 1.77 & 0.17 \\
\hline $\begin{array}{l}\text { Interrupting daily } \\
\text { routine }\end{array}$ & -1.5 & -1.0 & -1.0 & 2.02 & 0.13 \\
\hline $\begin{array}{l}\text { Uncertainty about } \\
\text { tostresultis }\end{array}$ & -1.9 & -2.4 & -1.8 & 1.65 & 0.19 \\
\hline Travelling time & .0 .8 & -0.3 & -0 . & 2.7 & 0.06 \\
\hline Judgmental attitude & -1.5 & -1.3 & -1.5 & 0.18 & 0.83 \\
\hline
\end{tabular}

In order to find out whether the difference in attitude aspects can be explained by a difference in gender, MANOVA was once more conducted at which, apart from intention to choice of circuits, gender was admitted as a separate factor. This analysis shows that there is no interaction effect between the factor 'intention to choice of circuits' and the factor 'gender'. This means, that females who intend to choose a specialist, GP or Family Planning Clinic respectively do not differ from each other in attifuce aspects. Likewise, the absence of an interaction effect means that males who intend to choose a specialist, GP or Family Planning Clinic, do not differ from each other in attitude aspects (Hotellings' $\mathrm{T}^{2}=.07 ; \mathrm{p<.001)}$. Females score higher on the attitude aspect 'option of a 
male or female physician' than males, regardless of intention to choice of circuits ( $F=14.4 ; p<.001)$. In Table 21 the main effect of the factor 'intention to choice of circuits' has already been discussed.

\subsection{Discussion}

In the Netherlands, persons who suspect an STD can get treatment from several providers and institutions. The results of this study show that virtually all adolescents know about one or more institutions for curative STD-health care. However, it is remarkable that $17 \%$ still does not recognise the GP as a provider in the field of STD. Likewise, it appears that the more specialised institutions such as MHSs and out-patient STD clinics are not well known among these adolescents. The fact that the so-called STD clinics are unknown among a very high percentage of these adolescents, might be caused by the unfamiliarity of the names of these type of facilities. It is therefore advisable to provide adolescents with information about the existence of and availability of several facilities for curative STD-health care in the provinces when educating them about venereal diseases. Furthermore, it is advisable to stress that STD clinics do exist and that one can get treatment there when one suspects an STD. When counselling about the local availability of STD facilities, extra attention should especially be paid to adolescents with a non-Dutch cultural background.

When it concerns the image of the availability of STD facilities in general, it is striking that attributes which concern the taboo on STDs, such as the possibility of anonymous treatment and the absence of a judgmental attitude by providers, still play an important role in STD health care. From a previous study among 825 persons with a request for an STD examination, it emerged that shame and the taboo on STDs can influence the decision not to go to their own GP (Leenaars, Rombouts \& Kok, 1993). This means that one needs to take into account the desired anonimity when organising STD-health care facilities in general and with regard to adolescents in particular. This way, an attempt can be made to avoid contact between potential (STD) patients when designing the waiting room and organising operating hours. Moreover, it may be pointed out that physicians have professional secrecy. When selecting providers for the prevention of STDs, the desired attitude of the provider towards sexual behaviour and STDs should be considered. In addition, it is advisable to not only implement these service attributes, but to also make them known. The fact that more than half of these pupils do not find it important that their GP get informed about the STD examination, probably has to do with the fact that the majority of the pupils eventually plan to go to their GP.

The majority of the adolescents expect to be treated afler school, get free treatment and they also expect to be able to choose a male or female physician. This means that evening and/or weekend operating hours are necessary for 
adolescents. Practically speaking, free treatment is already available at STD clinics and a couple of MHSs. Moreover, the medical insurance companies reimburse diagnosis and treatment of STDs with a GP. Only those who are privately insured with own-risk and those who are not insured, will have to pay for their consultation, diagnosis and treatment with their GP themselves. Considering the fact that the low-threshold facilities are relatively unknown, it seems sensible to pay extra attention to specific service attributes (such as the opportunity of anonymous and free treatment) of these institutions, when counselling. The significance of taking on both female and male physicians at institutions for curative STD-health care is confirmed by the results of this study. Beliefs and evaluations of the adolescents about the existence of service attributes which result in a long investment of time lead to the fact that long waiting and travelling times must be prevented. An appointment system can possibly solve the problem, as these pupils do not mind having to make an appointment beforehand. Improving the accessibility by public transportation is also possibility, as well as distributing services for curative STD-health care all over town.

When these pupils suspect an STD, most of them intend to go to their GP and a remarkably low percentage intends to go to a more specialised institution. In Fishbein and Azjen's (1975) model, it is assumed that the intention to behaviour (in this case seeking medical examination of an STD) can be explained by the attitude towards the behaviour on the one hand, and the expected pressure from the social environment on the other. In this model, the intention to behaviour is considered a motivational factor and immediate determinant of actual behaviour. However, research shows that habits, previous experiences with this behaviour (Azjen, 1985; Leenaars ot al. 1991), personal effectiveness (Kok \& Oostveen, 1987) and the presence of behavioural alternatives (Azjen \& Fishbein, 1980; Petty \& Cacioppo, 1981) are factors that can make an important contribution in addition to the prediction of the intention to behaviour. Whether the intention to behaviour everitually leads to the implementation of the actual behaviour is, according to Azjen (1985) and Kok \& Oostveen (1987), also determined by a number of non-motivational factors, such as the presence of barriers and the accessibility of resources and capabilities.

Considering the subject of research, the study population (has little to no experience with request for STD examination) and the measuring methods used, variables such as: habits, previous experiences, personal effectiveness, intention to behavioural alternatives and unexpected barriers can not be admitted as separate factors in this study. The role of these factors in the ultimate choice of an institution/provider is as yet unknown. However, it has been established that the intention to choice of circuits can be explained through beliefs and evaluations about several service attributes. Males who intend to go to a Family Planning Clinic expect more strongly than those who 
will choose the GP and 'specialist', that their requests for STD assistance will stay unknown to their GP, to their farnily and friends, and to the medical insurance companies. Moreover, they also consider this anonimity more important. These findings support the significance of the option that diagnosis and treatment for STDs also take place at Family Planning Clinics. However, this desired anonimity can have a impeding effect on conducting and implementing successful partner notification for males who will go to a Family Planning Clinic. Considering the results, it is also desirable to not refer adoles. cents at Family Planning Clinics to their own GP. Also the presence of male and female physicians and beliefs about personal treatment are important service attributes that contribute towards adolescents choosing the Family Planning Clinic when suspecting an STD. Apart from these service attributes, the unfamiliarity with STD clinics and the MHSs as institutions for curative STD-health care possibly play a role with the intention to choose a Family Planning Clinic.

The assumption that females intend to go to a Family Planning Clinic more often because of service attributes such as: anonimity and the option of a male or female physician, is not confirmed by these findings. In general, fernales do expect, more so than males, that male and female physicians will be present at the institutions of their choice. The fact that females intend to go to a Family Planning Clinic more often, probably has to do with the image of the Family Planning Clinics as institutions where one can go to with questions regarding sexuality and contraception.

If the research results, especially the results concerning the unfamiliarity with services and the image of several services, can be generalised to adolescents from other cities or to adolescents living in rural areas, however, remains to be seen and seems, ofcourse, to be dependent of the organisation and structure of provision of services in a specific region.

A limitation in the method of research is especially expressed in the analyses into the role of service attributes with the intention to choice of circuits. We have asked the respondents to indicate to what extent they expect a number of service attributes to be available at the institution/provider of their choice, but not to what extent they expect these same attributes to be available with other providers. The research method chosen results in the fact that no definite answer can be given about the contrast between the three subgroups and also leaves room for alternative explanations.

In short, the findings of this study boil down to what education about curative STD-health care for adolescents is definitely necessary. In order to relise a prompt medical examination of adolescents suspecting an STD, we advise the 'ideal care facilities' in a region to meet the following demands: anonymous opportunities for treatment, a non-judgmental attitude of the providers, an even distribution of male and female physicians who deal with curative prevention of STDs, evening and/or weekend operating hours, opportunity for free treatment, 
consultations by appointment, an even distribution of low-threshold facilities in the region, good accessibility of services and personal attention to a medical help question. Ofcourse, the quality of medical care should be quaranteed. Considering this diversity in expectations and demands of potential STDpatients it is therefore, according to us, desirable to strive for a pluriform provision of assistance. By using already existent infrastructures (Family Planning Clinics, MHSs, STD clinics, GPs and independent dermatologists) in which cooperation and referral patterns are established, the curative prevention of STDs can get an effective structure which is reliable and financially responsible. 


\section{REFERENCES}

Adler, N.E., Kegeles, S.M., Inwin, C.E. \& Wibblesman, C. (1990). Adolescent contraceptlve behavior: an assessment of decision processes. Journal of Pediatrics, 116, 463-471.

Ajzen, I. (1985). From intentions to actions: A theory of planned behavior. In J. Kuhl \& J. Beckmann (Eds), Action control: From cognition to behavior (pp. 11-40). Berlin: Springer-Verlag.

Ajzen, I. (1987). Attitudes, traits and action: dispositional prediction of behavior In personality and social psychology. In L. Berkowitz (Ed), Advances in Experimental Social Psychology. Volume 20. New York: Academic Press.

Ajzen, I. \& Fishbein, M. (1980). Understanding attitudes and predicting social behaviour. Englewood Cliffs: Prentice Hall.

Ajzen, I. \& Madden, T.J. (1986). Prediction of goal-directed behavior: attitudes, intentions and perceived behavioral control. Journal of Experimental Social Psychology, 22, 453 474.

Allard, R. (1989). Beliefs about AIDS as determinants of preventive practices and of support of coercive measures. American Journal of Public Health, 79, 448-452.

Altman, N.A. \& Ostrow, D.G. (1980). Risk factor identification for STDs In gay men. Paper presented at the meeting of the American Public Health Association, Detroit.

Aral, S.O., Mosher, W.D. \& Cates, W. (1991). Self reported Pelvic Inflammatory Disease in the United States, 1988. Journal of the American Medical Association, 266(18), 2570-2573.

Aral, S.O., Mosher, W.D. \& Cates, W. (1985). Self reported Pelvic Inflammatory Disease in the United States: a common occurrence. American Journal of Public Health, 75, 1216-1218.

Armonker, R.G. (1980). What do teens know about the facts of life? Journal of School Health, 50, 527-531.

Baldwin, J.D. \& Baldwin, J.I. (1988). Fecters affecting AIDS rolated esxual rick taking behaviors among college students. The Journa! of Sex Fesearch, 25, 181-196.

Bandura, A. (1982). Self-efficacy mechanism in human agency. American Psychology, 37, $122-147$.

Bandura, A. (1986). Soclal foundations of thought and action: a social cognitive theory. Englewood Clifis: Prentice Hall.

Bandura, A. (1989). Self-efficacy mechanism in physilogical activation and health promotion behavior. In J. Madden, S. Matthyse \& J. Barchas (Eds), Adaption, tearning and affect. New York: Raven Press.

Bauman, L.J. \& Siegel, K. (1987). Misperceptions among gay-man of the risk for AIDS asscciated with their sexual behavior. Journal for Applled Soclal Psychology, 17, 329. 350.

Basen-Engquist, K. (1992). Psychosocial predictors of "safer sex" behaviors in young adults. AIDS Education and Prevention, $4(2), 120-134$.

Becker, M.H. (1974). The Health Belief Model and personal health behavior. Health Education Monograph, 2, 324-508.

Becker, M.H. \& Joseph, J.G. (1988). AIDS and behavioral change to reduce risk: a review. American Journal of Public Health, 78(4), 394-410. 
Boutellier, C.J. (1987). Enkele cijfers over prostitutie. Justitiele Verkenningen, 13(1), 36-44. Bownes, I.T., O'Gorman, E.C. \& Dinsmore, W. (1990). Factors influencing perception of risk of HIV aquisition among male heterosexual STD clinic attenders. Irish Journal of Psychological Medicine, 7, 94-100.

Braeken, D. (1988). Persbericht: jongerencampagne "wat ik je wil zeggen", Rutgers Stlchting: Den Haag.

Brown, L.K., DIClimente, R.J. \& Reynolds, L.A. (1991). HIV prevention for adolescents: utility of the health Belief Model. AIDS Education and Prevention, 3(1) 50-59.

Brubaker, R.G. \& Wickersman, D. (1990). Encouraging the practice of testicular selfexamination: a fleld application of the Theorie of Reasoned Action. Psychology and Health, 9(2), 154-163.

Brussa, L. (1987). De bultenlandse prostituee. In F. Belderbos \& J. Visser (Eds.). Beroep: Prostituee, Utrecht: Stichting Welzijns Publicaties.

Calnan, M. (1987). Health and IIIness; the lay perspective. London: Tavistock Publications.

Calnan, M. \& Moss, S. (1984). The Health Belief Model in compliance with education given at a class in breast self examination. Journal of Health and Social Behavior, 25, 198 210.

Calnan, M. \& Rutter, D. (1986). The health beliets predict health behavior: An analysis of breast self-examination. Social Sclence and Medicine, 22, 673-678.

Cassee, E. TH. (1973). Naar de Dokter. Meppel.

Catania, J.A., Dolcini, M.M., Coates, T.J., Kegeles, S.M., Greenblatt, R.M. \& Puckett, S. (1989). Predlctors of condom use and multiple partnered sex among sexually active adolescent women: Implications for AIDS related health interventions. The Journal of Sex Research, 26(4), 514-524.

Catanla, J.A., Kegeles, S.M. \& Coates, T.J. (1990). Towards an understanding of risk behavior: an AIDS risk reduction model. Health Education Quarterly, 17, 53-72.

Cattarrall, R.D. (1984). National Strategies for STD control: A U.K. perspective In K.K. Holmes, P.A. Mardh, P.F. Sparling \& P.J. Wienser (Eds). Sexually Transmitted Diseases, New York: McGraw-Hill.

Cates, W., Rolfs R.T. \& Aral, S.O. (1990). Sexually Transmitted Diseases, Pelvic Inflammatory Disease and infertility: an epidemiologic update. Epidemiological Roviews, 12, $199-220$.

Cates, W. (1984). Sexually transmitted organisms and infertility: the proof of the pudding. Sexually Transmitted Diseases, 11, 113-116.

Cates, W. \& Meheus, A. (1990). Strategies for development of sexually transmitted diseases control programs. In K. Holmes, P.A. Mardh, P.F. Sparling, P.J. Wiesner, W. Cates, S.M. Lemon \& W.E. Stamm (Eds), Sexually Transmitted Diseases. sec. edition. New York: Mc.Graw-Hill Book Company.

Cates, W. \& Raugh, J.L. (1985). Adolescence and sexually transmitted diseases: an expanding problem. Journal of Adolescent Health Care, 6, 257-261.

Centers for Disease Control (1980). Venereal Disease Control Division, SSS, Atlanta GA 30333.

Centers for Disease Control (1988). Number of sex partners and potential risk for sexual exposure to Hurnan Immunodeficiency Virus. MMWJ, 37, 565-568. 
Chacko, M.R. Lovchik, J.C. (1986). Chlamydia trachomatis infections in sexually active adolescents: prevalence and risk factors. Pediatrics, 73, 836-840.

Champion, V.L. (1990). Breast Self Examination in women 35 and older: a prospective study. Joumal of Behavioral Medicine, 13 (6), 523-538.

Champion, V.L. (1985). Use of the Health Belief Model in delermining the frequency of breast self examination. Research Nursing and Health, 8(4), 373-379.

Chapman, S., Stoker, L., Ward, M., Porritt, D. \& Fahey, P. (1990). Discriminant attitudes and beliefs about condoms in young multi-partner heterosexuals. International Journal of STD and AIDS, 1, 422-428.

Chapman, S. \& Hodgson, J. (1988). Showers in raincoats: attitudinal barriers to condom use in high-risk heterosexuals. Community Health Studies, 12, 97-105.

Cleary, P.D., Mechanic, D. \& Greenley, J.R. (1982). Sex differences in medical utilisation: an empirical Investigation. Journal of Health and Social Behavior, 23, 106-118.

Cleary, P.D., Rogers, T.F., Singer, E., Avorn, J., Van Devanter N., Perry, S. \& Pindyck, J. (1986). Health education about AIDS among seropositive blooddonors. Health Education Quarterty, 13(4), 317-329.

Cochran, S.D. \& Peplau, L.A. (1991). Sexual risk reduction behaviors among young heterosexual adults. Social Science and Medicine, 33(1), 25-36.

Cody, R. \& Lee, C. (1990). Behaviors, beliefs, and intentions in skin cancer prevention. Journal of Behavioral Medicine, 13 (4), 373-389.

Coutinho, R.A., Rijsdijk, A.J., Hoek, J.A.R van den \& Leentvaart-Kuiper, A. (1992). Decreasing incidence of PID in Amsterdam, Genitourinary Medicine, 68, 353-355.

Coutinho, R.A., Schoonhoven, J.F., Hoek, J.A.R. van den \& Emsbroek, J.A. (1987). Influence of special surveillance programmes and AIDS on the declining incidence of syphilis in Amsterdam. Genitourinary Medicine, 63, 210-213.

Cvetkovich, G. \& Grote, B. (1981). Psychology and contraceptive use: an investigation of decision making and communication skills. Journal of Pediatrics, 211-225.

D'Costa, L.J., Plummer, F.A., Bowmer, J., Franser, L., Pilot, P. \& Ronald, A.R. (1985). Prostitutes as a major reservoir of sexually transmitted diseases in Nairobi, Kenya. Sexually Transmitted Diseases, 12, 64-67.

Darrow, W.W. (1987). Risk factors for Human Immunodeficiency Virus infections in homosexual men. American Journal of Public health, 77, 479-483.

Darrow, W.W. \& Pauli, M.L (1985). Health Behavior and Sexually Transmitted Diseases. In K.K. Holmes (Eds.). Sexually Transmilted Diseases. New York: McGraw-Hill.

Darrow, W.W. Siegel, K. (1990). Preventive health behavior and STDs. In K.K. Holmes, P.A. Mardh, P.F. Sparling, P.J. Wiesner, W. Cates, S.M. Lemon \& W.A. Stamm (Eds), Sexually Transmitted Diseases. New York: McGraw-Hill.

De Schrijver, A. \& Meheus, A. (1990). Review/analyses: epidemiology of Sexually Transmitted Diseases: the global picture. Bulletin of the World Health Organisation, 68(5), 639-654.

Deenen, A. \& Naerssen, L. van (1988). Een onderzoek naar enkele aspecten van de homoseksuele identiteitsontwikkeling. Tijdschrift voor Seksuologie, 12, 105-116.

Delil, M.J. (1988). Een exploratief onderzoek naar kennis, attitudes en gedrag ten aanzien van AIDS en het voorkomen van besmetting met HIV bij leerlingen van het $L B B$ en Mavo. Unpublished report, Easisgezondheidsclenst Ostelijk Zuid Limburg, Heerlen. 
DiClemente, R., Forrest, K. \& Mickler, S. (1990). Differential effects of AIDS knowledge and perceived susceptibility on the reduction of high risk sexual behaviors among college adolescents. Proceedings of the Vth International conference on AIDS 4-9 juni, 1990, Montreal: Abstract number TDP92.

DiClimente, R.J., Boyer, C.B. \& Morales, E.S. (1988). Minorities and AIDS: knowledge, attitudes and misconceptions among Black and Latino adolescents. American Journal of Publlc Health, 78, 55-57.

DiClimente, R.J., Zorn, J. \& Temoshok, L (1986). Adolescents and AIDS: a survey of knowledge, beliefs and attitudes about AIDS in San Fransisco. American Journal of Public Health, 76, 324-331.

Eardley, A. (1974). Triggers to action: a study of what makes women seek advice for breast conditions. International Journal of Heahth Education, 17, 256-265.

Ekstrand, M.L \& Coates, T.C. (1990). Maintenance of safer sexual behavior and predictors of risky sex: the San Fransisco men's health study. American Joumal of Public Health, 80, 973-977.

Emmons, C., Joseph, J., Kessler, R., Wortman, C., Montgomery, S. \& Ostrow, D. (1986). Psychosoclal predlctors of reported behavior change in homosexual men at risk for AIDS. Health Education Quarterly, 13, 331-345.

Fishbein, M. \& Ajzen, I. (1975). Belief, attitude, intention and behavior: an introduction to theorie and research, Addisson Wesley: Reading Mass.

Franklin, F.N. \& Stolz, E. (1984). Organization of clinical facilities for STD control. In K.K. Holmes, P.A. Mardh, P.F. Sparling \& P.J. Wiesner (Eds). Sexually Transmitted Diseases. New York: McGraw-Hill.

Friedland, G.H. \& Klein, R.S. (1987). Transmission of the hunman immunodeficiency virus. New England Journal of Medicine, 317, 1125-1135.

George, H. (1989). Counselling people with AIDS, their lovers, friends and relations. In J. Green \& A. McCreamer (Eds), Counselling in HIV infections and AIDS. London: Blackwell Publishers.

Geneeskundlge Hoofdinspectie van de Volksgezondheid (GHI) (1990). Aangifteciffers van gonorroe en syfilis. Nederlands Tijdschrift voor Geneeskunde, 134, 1008

Geneeskundige Hoofdinspectie van de Volksgezondheid (GHI) (1993). Anonieme registraties AIDS diagnosis, Continue registratie. GHI, Rijswijk.

Geneeskundige Hoofdinspektie van de Volksgezondheid (1992). Registratie niet curatieve SOA-bestrljding bij GGD'en. Landelijke tabellen, 1986-1990.

Geslachtsziektenbestrijding GGD Amsterdam (1990), jaarverslag.

Gieling. R. A. \& Heuzer, A. (1988). AIDS op school; een onderzoek naar kennis en attitudes ten aanzien van AIDS bij leerlingen in het voortgezet onderwijs in de regio Amsterdam. Unpublished Report, Hogeschool Holland, Amsterdam.

Gilchrist, L.D. \& Schinke, S.P. (1983). Coping with contraception: Cognitive and behavioral methods with adolescents. Cognitive Therapy and Research, 7, 379-388.

Gottlieb, N.H., Vecalis, T.D., Palmer, D.R. \& Collon, R.T. (1988). AIDS related knowledge, attitudes behaviors and intentions among Texas college students. Health Education Research, 3, 67-73. 
Graaf, R. de, Vanwesenbeeck, I., Zessen, G.J. van, Straver, J.C. \& Visser, J.H. (1992). Prostitutie, seksueel gedrag en de mogelijke verspreiding van HIV. Tijdschrift voor Sociale Gezondheidszorg, 5, 12-20.

Green, LW. \& Kreuter, M.W. (1991). Health Promotion Planning: an educational and environmental approach. Mountain View: Mayfield Publishing Company.

Greenblatt, R.M., Lukehart, S.A. \& Plummer, F.A. (1988). Genital ulcerations as risk factor for Human Immunodeficiency Virus Infection. AIDS, 2, 47-50.

Greenfield, L.G., Bigelow, G.E. \& Brooner, R.K. (1992). HIV risk behavior in drugusers: increased blood booting during cocaine injection. AIDS Education and Prevention, 4(2), 95-107.

Griensven, G.J.P. van, Vroome E.M.M. de, \& Tielman, R.A.P. (1987). Risk factors and prevalence of HIV antibodies in homosexual men in the Netherlands. American Joumal of Public Health, 125, 1048-1057.

Griensven, G.J.P. van, Tieiman, R.A.P., Goudsmit, J., Noordaa, J. van der, Wolf, F. de, \& Coutinho, R.A. (1986). Risicofactoren en prevalentie van LAV/HTLVIII antistoffen bij] homoseksuele mannen in Nederland. Tijdschrit voor de Sociale Gezondheidszorg, 64, 100-107.

Griensven, G.J.P. van, Tielman, R.A.P. \& Goudsmlt, J. (1987). Risk factors and prevalence of HIV antibodies in homosexual men in the Netherlands. American Journal of Public Health, 125, 1048-1057.

Haastrecht, H.J.A. van, Hoek, J.A.R. van den, \& Coutinho, R.A. (1991). Aanwijzingen voor veiliger seksueel gedrag bij heteroseksuelen in Amsterdam onder invloed van AIDS. Tijdschrift voor Sociale Gezondheidszorg, 69, 3-8.

Handsfield, H.H., Jasman, L.L., Roberts, P.L., Hanson, V.W., Kothenbeutal, R.L. \& Stamm, W.E. (1986). Criteria for selective screening for chlamydia trachomatic infection In women attending family planning clinics. Journal of the American Medical Assoclation, 255, 1730-1734.

Hansen, W.B., Hahn, G.L. \& Wolkenstein, B.H. (199). Perceived personal immunity: beliefs about susceptibility. The Journal of Sex Fesearch, 24, 622-628.

Harrison, R.M. (1982). Women's treatment decisions for genital symptoms. Journal of the Royal Society of Medicine, 75, 23-26.

Hart, G. (1976). Sexually Transmitted Diseases. In J.J. Head (Ed.), Carolina Biology readers. Burlington N.C.: Carolina Biology Supply Co.

Haverkos, H.W., Bukoski, W.J. \& Amsel, Z. (1989). The initiation of male homosexual behavior. Joumal of the American Medical Association, 262(4), 501.

Hibbard, J.H. (1984). Sex differences in health and illness orientation. Int. Q. Commun. Health Education, 4, 95-104.

Hickson, F., Weatherburn, P., Davies, P.M., Hunt, A.J., Coxon, A.P.M. \& McManus, T.J. (1992). Why gay men engage in anal intercourse, oral presentation, Eight international conference on AIDS, Amsterdam.

Hingson, A., Strunin, L \& Berlin, B. (1987). Acquired Immune Deficiency Syndrome: changes in knowledge and behaviors among teenagers, Massachusetts Statewide Surveys, 1986-1988. Pediatrics, 85, 24-29. 
Hingson, R.W., Strunin, L., Berlin, B.M. \& Heeren, T. (1990). Beliefs about AIDS, use of alcohol and drugs and unprotected sex among Massachusetts adolescents. American Journal of Public Health, 80, 295-299.

Hoek, J.A.R. van den, Haastrecht, H.J.A. van, Henquet, C.J.M. \& Coutinho, R.A. (1993). Toename van gonorroe bij jongere homoseksuele mannen in Amsterdam. Nederlands Tijdschrift voor Geneeskunde, 137(3), 144-145.

Hoek, J.A.R. van den, Fennerna, J.S.A., Haastrecht, H.J.A. van, Doornum, G.J.J. van, Henquet C.J.M. \& Countinho R.A. (1992). HIV prevalentie onder bezoekers van een polikliniek voor seksueel overdraagbare aandoeningen en bij een groep prostituees en prostituanten. Nederlands Tijdschrift voor Geneeskunde, 136, 1813-1818.

Hoek, J.A.P. van den, Griensven, G.J.P. van \& Coutinho, R. (1990). Aanwijzingen voor een toename van onveilig seksueel gedrag bij homoseksuele mannen in Amsterdam. Nederlands Tljdschrift voor de Geneeskunde, 134, 1229-1230.

Holmes, K.K. (1990). Sexually Transmitted Diseases. In K. Holmes, P.A. Mardh, P.F. Sparling, P.J. Wiesner, W. Cates, S.M. Lemon \& W.E. Stamm (Eds). Sexually Transmitted Diseases, sec. ed. New York: McGraw-Hill.

Holmberg, S.D., Stewart, J.A. \& Russell, A. (1987. Prior herpes simplex type 2 infections as a risk factor for HIV infection. Journal of the American Medical Association, 259, 1048-1050.

Hoof, A. van, \& Laga, M. (1991). HIV-infectie en SOA, wederzijdse beinvloeding. SOA Bulletin, 12(6), 6-9.

Hooykaas, C., Pligt, J. van der, Doornum, G.J.J. van, Linden, M.M.D. van der \& Coutinho, R.A. (1989). Heterosexuals at risk for HIV: differences between private and commercial partners In sexual behavior and condom use. AIDS, 3, 525-532.

Hopman-Rock, M., Danz, M.J., Visser, A.C.B. \& Vogels, T. (1990). Kennis en houding ten aanzien van AIDS. In T. Vogels \& R. van der Vliet (Eds.), Jeugd en Seks. (pp 152. 169), Den Haag, SDU.

Houweling, H., Jager, J.C., Coutinho, R.A., Bijkerk, H. \& Ruitenberg, E.J. (1987). Epldemlologle van AIDS en HIV infecties in Nederland: huidige situatie en prognose voor de periode 1987-1990. Nederlands Tijdschrift voor Geneeskunde, 131 (19), 818 824.

Houweling, H. \& Jager, J.C., (1993). HIV infecties en AIDS. In M.J.W. van de Laar (Ed), SOA in Nederland. Interne publicatie, Bilthoven, RIVM.

Howard, J.A. \& Sheth, J.N. (1969). The theory of buyer behavior. New York, John Wiley. Jaccard, J. \& Davidson, A.P. (1972). Toward an understanding of family planning behaviors: an initial investigation. Journal of applied Social Psychology, 2, 228-235.

Jager, J.C., Poos, M.J.J.C., Houweling, H., Postema, C.A. \& Coutinho, R.A. (1990). Prognose aangaande HIV infecties en AIDS epidemie in Nederland op basis van wiskundige analyse. Nederlands Tijdschrift voor Geneeskunde, 134, 2486-2491.

James, N.J., Gillies, P.A. \& Bignell, C.J. (1990). AIDS related risk perception and the sexual behavior among sexually transmitted disease clinic attenders. International Journal of STD \& AIDS, 2, 264-271.

Jonkers, R., Liedekerken, P.C., Haes, W.F.M. de, Kok, G.J. \& Saan, J.A.M. (1988). Effectlviteit van Gezondheidsvoorlichting en Opvoeding (GVO). Den Haag: Care. 
Janz, N.K \& Becker, M.H. (1984). The Health Belief Model: a decade later. Health Education Quarterty, 11, 1-47.

Joseph, J., Montgomery, S., Emmons, C., Kessler, R., Ostrow, D., Wortman, C., O'Brien, M. \& Eshleman S. (1987). Magnitude and determinants of behavioral risk reduction: longitudinal analysis of a cohort at risk for AIDS. Psychology and Health, 1, 73-96.

Kegeles, S.M., Adler, N.E. \& Inwin, C.E. (1989). Adolescents and condoms; association of beliefs with intention to use. American Journal Dis. Child, 143, 911-915.

Kegeles, S.M., Adler, N.E. \& Irwin, C.E. (1988). Sexually active adolescents and condoms: changes over one year in knowiedge, attitudes and use. American Journal of Public Health, 78, 460-461.

Keller S.E., Schleiffer, S.J., Bartlett, J.A. \& Johnson, R.L. (1988). The sexual behavior of adolescents and risk of AIDS. Journa/ of the American Medical Association, 260, 3586 3590.

Kelly, J.A., ST. Lawrence, J.C., Hood, H.V. \& Brasfield, T.L. (1989). Behavioral intervention to reduce AIDS risk activities. Journal of Consutting and Clinical Psychology, 8, 21-26.

Kessler, R. (1986)). Sex differences in the use of health services. In S. McHugh, T.M. Vallis (Eds). Illness behavior: A Multidisciplinary Model. New York: Plenum Press.

Kok, G.J. (1988). Health motivation, health education from a social psychological point of view. In S. Maes, C.D. Spielberger, P.B. Defares \& I.G. Sarason (Eds). Topics in health psychology, New York: Wiley.

Kok, G.J, Buisman, W., Kampschuur, P., Krist, L. \& Vange, P. van der (1981). Attltudes, sociale normen en gedrag. Nederlands Tljdschritt voor de Psychologie, 36, 57-63.

Kok, G.J., Vries, H. de, Mudde, A.N. \& Stretcher, V.J. (1991). Planned Health Education and the role of self-efficacy: Dutch research. Health Education Research, 6 (2), 231. 238.

Kok, G.J. \& Oostveen, T. (1987). Modellen ter verklaring van gezondheidsgedrag II: recente ontwikkelingen van belang bij GVO. GVO-Preventie, 4, 225-233.

Kok, G.J. \& Vries, H. de (1989). Primary prevention of cancers: the need for health education and intersectoral health promotion. In T. Heller, B. Davey \& L. Bailey (Eds), Reducing the risk of cancers (pp 99-111). London: Hodder \& Soughton.

Koopman, C., Rotheram, M.J., Henderson, R., Bradley, J.S. \& Hunter, J. (1990). Assessment of knowledge of AIDS and beliefs about AIDS prevention among adolescents. AIDS Education and Prevention, 2(1), 58-70.

Kroger, F. \& Wiesner, P.J. (1981). STD education: Challenge for the 80s. Journal of School Health, 51, 242-246.

Kuiken, C.J., Griensven, G.J.P. van, Vroome, E.M.M. de, \& Coutinho, R.A. (1990). Risicofactoren en sexuele gedragsverandering in voor antilichamen geseroconverteerde homosexuele mannen. Tijdschrift voor de Sociale Geneeskunde, 8, 329-334.

Laar, M.J.W. van de, Schellekens, J.F.P. \& Klingeren, B. van (1993). Gonorroe. In M.J.W. van de Laar (Ed). SOA in Nederland. Unpublished report, Bitthoven: RIVM.

Laar, M.J.W. van de, Sleutjes, M.P.M., Postema, C.A. \& van de Water, H.P.A. (1992). Seksueel overdraagbare aandoeningen bij allochtone bevolkingsgroepen; een orienterend onderzoek. Nederlands Tijdschritt voor Geneeskunde, 135, 34, 1542-1546.

Laar, M.J.W. van de, Pickering, J., Hoek, J.A.R. van den, Griensven, G.J.P. van, Coutinho, R.A., \& Water, H.P.A. van de, (1990). Declining gonorrhoea rates in the Netherlands, 
1976-1988: consequences for the AIDS epidemic. Genitourinary Medicine, 66, 148-155. Lashley, M.E. (1987). Predictors of Breast Self Examination practice among eldery women. Advanced Nursing Science, 35(3), 183-185.

Lee, N.C., Rubin, G.L. \& Borucki, R. (1988). The intrauterine device and Pelvic Inflammatory Disease revisited: new results from the Women's Health Study. Obstetric Gynecology, 72, 1-6.

Leenaars, P.E.M., Rombouts, A., Lammerdin, A.G. \& Zwetsloot, L.M. (1990). Opvattingen, evaluaties en preventie van Seksueel Overdraagbare Aandoeningen. Tijdschrift voor Gezondheidsbevordering, 11(2), 13-23.

Leenaars, P.E.M., Rombouts, R. \& Schrijvers, A.J.P. (1990). Bekendheid met voorzieningen voor geslachtsziektenbestrijding. SOA-bulletin, 11 (3), 10-12.

Leenaars, P.E.M., Oliehoek, A.J.M. \& Schrijvers, A.J.P. (1991). Regionale geslachtsziektenbestrljding: ver variaties op een thema. Unpublished report, nr. 91.23., Department of Health Science and Epidemiology, University of Utrecht, the Netherlands.

Leenaars, P.E.M., Rombouts, R., Lammerdin, A.G. \& Zwetsloot, L.M. (1991). Determinanten van condoomgebruik onder jongeren. Gedrag en Gezondheid, 19, (2) 77-86.

Leenaars, P.E.M., Rombouts, P., Lammerdin, A. G. \& Zwetsloot, L.M. (1992). Consumentengedrag van middelbare scholieren bij vermoeden van een Seksueel Overdraagbare Aandoening. Tijdschrift voor Sociale Gezondheidszorg, 70, 2, 95-102.

Leenaars, P.E.M., Rombouts, R. \& Kok, G.J. (1993). Seeking medical care for a sexually transmitted disease: determinants of delay behavior. Psychology and Health, 8, 17-32.

Leenaars, P.E.M, Rombouts, A. \& Kok, G. (1994). Service Attributes and the choice for STD Health services in persons seeking a medical examination for an STD. Social Science and Medicine, 8, 363-371.

Leenaars, P.E.M., Sandfort, Th. G.M., Oliehoek, A.J.M. \& Schrijvers, A.J.P. (in press). Psychosociale begeleiding bij de afname van een HIV test: ervaren noodzaak on tevredenheid. Gedrag en Gezondheid.

Lierop, G.S. van, Houweling, H., Wlessing, L.G. \& Katchaki, J.N. (1992). Surveillance van HIV Intectles in de regio Arnhem; deel 2: monitoring van laboratoriumdiagnostiek naar HIV Infecties, april 1991-juni 1991, Unpublished manuscript, RIVM Bilthoven.

Linden, M.M.D. van der, Velde, F.W. van der, Hooykaas, C., Doornum, G.J.J. van, \& Coutinho, R.A. (1990). HIV prevalentie onder heteroseksuelen met veel wisselende partners. Nederlands Tijdschrit voor Geneeskunde, 134, 28, 1361-1364.

Lindan, C., Kegeles, S. \& Hearst, N. (1990). Heterosexual behaviors and factors that influence condom use among patients attending a sexually transmitted disease clinic In San Fransisco, MMWR, 39, 685-689.

Lisking, L., Wharton, C. \& Blackburn, R. (1992). Condoms now more than ever. Population Report, 8, 1-36.

Lucas, D.B. \& Britt, S.H. (1963). Measuring advertising effectiviness. New York, Mc. Graw Hill.

Luijkx, J.B., Rademakers, J., Straver, C.J., Rijt, G.A.J. van der, \& Zessen, G. van (1991). Uitgangspunten voor onderzoek naar determinanten van condoomgebruik bij heteroseksuelen. Nederlands Tijdschrif voor de Psychologie, 46, 309-321. 
Luykx, J.B. Marsman, G., \& Rijt, G. van der, (1989). Het condoom: anticonceptie of profylactisch middel ter voorkoming van Sexueel Overdraagbare Aandoeningen. Gedrag en Gezondheid, 16, 4-10.

Luykx, J.B., Marsman, G.W. \& Rijt, G.A.J. van der (1987). De seksuele moraal: een verklarend gegeven voor kennis- en houdingsaspekten van geslachtsziekten. Gezondheid en Samenleving, 8(2), 108-119.

Luykx, J.B., Marsman, G.W. \& Rijt, G.A.J. van der (1988). De invloed van SOA voorlichting op consultaties. Tljdschrif voor Sociale Gezondheidszorg, 66, 24-76.

MacDonald, N.E., Wells, G.A., Fisher, W.A., Warren, W.K. King, M.A., Dohertey, J.A. \& Bowie, W.R. (1990). High risk STD/HIV belhavior among college students. Journal of the American Medical Association, 263, 23, 3155-3159.

Maiman, LA. Becker, M.H. (1974). The Health Belief Model: origins and correlates in psychological theorles. Health Education Monographs, 2, 336-353.

Marchbanks, P.A., Lee, N.C. \& Peterson, H.B. (1990). Cigarrette smoking as a risk factor for Pelvic Inflammatory Disease. American Journal Obstetrics and Gynocology, 162, 639-644.

Massey, V. (1986). Perceived susceptibility to breast cancer and practice of breast self exarnination. Nursing Research, 35(11), 183-185.

McCormach, W.M., Rosner, B. \& McComb, D.E. (1985). Infection with Chlamydia trachomatis in women college students. American Journal of Epldemiology, 121, 107 113.

McCusker, J., Zapka, J.G., Stoddard, A.M. \& Mayer, K. (1989a). Responses to the AIDS epldemic among homosexually active men: factors associated with preventive behavior. Patient Education and Counselling, 13, 15-30.

McKusick, L., Coates, T.J., Wiley, J., Morin, S. \& Stall, R. (1987). Prevention of HIV infection among gay and bisexual men. Paper presented at the Third International Conference on AIDS, Washington DC.

McKusick, L., Hortsman, W. \& Coates, T.J. (1985). AIDS and the sexual behavior reported by gay men in San Francisco. American Journal of Public Health, 75, 493-496.

McKussick, L., Coates, T.J. \& Morin, S.F. (1990). Longitudinal predictors of reductlons in unprotected anal intercourse among gay men in San Fransisco: The AIDS Behavioral Research Project. American Journal of Public Health, 80, 978-983.

Mechanic, D. (1982). The epidemiology of illness behavior and its relationship to physical and psychological distress. In D. Mechanic (Ed). Symptoms, illness behavior, and helpseeking, (pp 1-25), New Brunswick NJ: Rutgers University Press.

Mechanic, D. (1986). Illness behavior: an overview. In S. McHugh and T.M. Vallis (Eds). IIInessbehavior: a multidisciplinary Model (pp 101-110). New York: Plenum press.

Meheus, A. (1990). The spectrum of Sexually Transmitted Diseases and approaches for their control. In M. Paalman (Ed) Promoting Safer Sex. (pp 17-24). Amsterdam/Lisse, Zwets and Zeitlinger.

Mens, L.P.M. van, (1989). De invloed van de vrij veilig campagne op condoomgebruik door prostituanten. Maandblad voor de Geestelijke Volksgezondheid, 44, 7/8, 474-486.

Meyer, A. (1988). Keynote address at the second International Youth Services Conference, Chicago. 
Miltenburg, H.M.Th.M., Paalman, M.E.M. \& Reus, J.Th.N.M. (1985). Gonorroe in Nederland. Report, Utrecht SOA Stichting.

Montgomery, S.B., Joseph, J.G., Becker, M.H., Ostrow, D.G., Kessler, R.C. \& Kirscht, J.P. (1989). The Health Belief Model in understanding compliance with preventive recommendations for AIDS: How useful? AIDS Education and Prevention, 1, 303-323.

Mullen, P.D., Green, L.W. \& Persinger, G. (1985). Clinical trials of patient education for chronic conditions; a comparative meta analysis of intervention types. Preventive Medicine, 14, 753-781.

Munday, P.E. (1990). Genitourinary Medicine services: consumer's view. Genitourinary Medicine, 66, 108-111.

Munik, G.Y. (1989). Condoms and Hepatitis B Virus infection. In: Condoms in the prevention of Sexually Transmitted Diseases. The proceedings of a conference of the American Social Health Association.

Murphy, J.S. (1990). The condom industry in the United States, Jefferson, North Carolina, McFarland.

Nationale Commissie AIDS Bestrijding (1990A). AIDS en prostitutie; bijgestelde notitie AIDS en prostitutie 1988, Amsterdam.

Norusis, M.J. (1987). SPSSPC+ 3.1. Marketing department SPSS Inc., Chicago, Illinois.

Norusis, M.J. (1988). SPSS/PC for the IBM PC/XT/AT. Marketing department SPSS Inc. Chicago, Illinois.

Notermans, M. (1987). Kennis en attitude ten aanzien van AIDS: een enquete onder middelbare scholleren in Maastricht. Tijdschrift voor de Sociale Gezondheidszorg, 65, 765-766.

Orduna, B.C. (1980). Time delays critical to gonorrhea. Polk County Medical Bulletin, 51, 79-82.

Paavonen, J., Geisala, K. \& Heinonen, P.K. (1987). Microbiological and histological findings in acute Pelvic Inflammatory Disease. British Journal Obstetrics and Gynaecology, 94, 454-460.

Paulussen, T., Kok, G. Knibbe, R.A. \& Cramer, A. (1990): Determinanten van aan AIDS gerelateerde risicogedragingen van intraveneuze druggebruikers. Tijdschrift voor de Sociale Gezondheidszorg, 68, 129-136.

Peeters, M.F., Bots, A.S., Merkus, J.M. \& Prak, F.M. (1985). Chlamydia Trachomatis, Infertiliteit en in vitro-fertilisatie. Nederlands Tijdschrift voor Geneeskunde, 31, 14381442.

Pennebaker, J.W. (1982). The psychology of physical symptoms. New York, Springer.

Petty, R.E. \& Cacioppo, J.T (1981). Attitudes and persuasion: classic and conternpary approaches. Dubuque: Brown.

Pligt, J. van der (1991). Risicoperceptie, onrealistisch optimisme en AIDS-preventief gedrag. Nederlands Tijdschrift voor de Psychologie, 46, 22.237.

Plummer, F.A. \& Ngugi, E.N. (1990). Prostitutes and their clients in the epidemiology and control of sexually transmitted disease. In K.K. Holmes, P.A. Mardh, P.F. Sparling and P.J. Wiesner (Eds.). Sexually Transmitted Diseases (pp 71-76). 2nd ed, New York, NY: McGraw-Hill.

Polit-O'Hara, D. Kahn, J. (1985). Communication and contraceptive practices in adolescent couples. Adolescence, 20, 33-43. 
Quinn, T.C. (1987). Screening for Human Immunodeficiency Virus (HIV) infection in patients attending sexually transmitted disease clinics: riskfactors and correlates of infections (abstract 137 Wereld congres SOA in Atlanta).

Reinking, D.P., Postma, M.J., Albers, C.E.S., Boom, F.M.L.G. van den \& Jager J.C. (1992). AIDS in Nederland tot 2000. Houten: Bohn Stafleu, Van Loghurn.

Reuben, N., Hein, K. \& Drucker, E. (1988). The relationship of high risk behaviors to AIDS knowledge in adolescent high school students. Presented at the Annual Conference of the Society for Adolescent medicine, New York.

Rice, P.A. \& Schachter, J. (1991). Pathogenesis of Pelvic Inflammatory Disease: What are the questions?. Journal of the American Medical Association, 266, 18, 2587-2593.

Richard, R., Pligt, J. van der \& Vries, N. de, (1991). De invloed van gewoontes, persoonlijke effectiviteit en gevoelsmatige reacties op condoomgebruik bij jongeren. Gedrag en Gezondheid, 19(2) 65-76.

Rogers, R. W. (1983). Protection Motivation Theory of fear appeals and attitude change. The Journal of Psychology, 91, 93-114.

Rolts, R.T., Galaid, E.I. \& Zaidi, A.A. (1990). Epidemiology of Pelvic Inflammatoy Disease: trends in hospitilization and office visits, 1979-1988. In: Proceedings of the CDC and National Institute of Health joint meeting. PID: Prevention, Management and Research In the 1990s. Betheseda, MD.

Roosendaal, F.R., Smit, C., Varekamp, I., Brocker, A., Dijck, H. van, Suurmeyer, T.P.B. \& Briet, E. (1988). Hemofilie in Nederland 3: verslag van een in 1985 gehouden landelijk onderzoek onder mensen met hemofilie. RUL/RUG, Leiden/Groningen, orgaan van de Nederlandse vereniging voor hemofiliepatienten.

Rosenstock, I. (1974). Historical origins of the Health Belief Model. Health Educatlon Monographs, 2, 328-335.

Rosenstock, I.M., Strecher, V.J. \& Becker, M.H. (1988). Social Learning theorie and the Health Belief Model. Health Education Quarterly, 15, 175-183.

Ross, M.W. (1988). Attitudes towards condoms as AIDS prophylaxis in homosexual men: dimensions and measijrement. Psychology and Health, 2, 291-299.

Ross, M.W. (1990). Psychovenereology: psychological aspects of AIDS and the other Sexually Transmitted Diseases. In D.W. Ostrow (Ed). Behavioral aspects of AIDS. New York: Plenum Medical Book Company.

Ross, M.W. (1990). Psychological determinants of increased condom-use and safer sex in homosexual men: a longitudinal study. International Journal of STD and AIDS, 1, SB101.

Rotter, J.B., Chance, J.E. \& Phares, E.J. (1972). Applications of a social learning theory of personality. New York: Holt, Rinehart and Winston Inc.

Rutledge, D. (1987). Factors related to women's practice of Breast Self Examination. Nursing Research, 36, 117-121.

Sandfort, Th.G.M., Vroome, E. de, Paalman, M. \& Tielman, R. (1989). Condooms in Nederland, determinanten van huidig on toekomstig gebruik. Unpublished report, Interfacultaire Werkgroep Homostudies en SOA-stichting, Utrecht.

Schaalma, K Kok, G. \& Peters, L (1993). Determinants of consistent condom use by adolescents: the impact of experience with sexual intercourse. Health Education Research, 8(2), 1-15. 
Schachter, J., Stoner, E. \& Moncada, J. (1983). Screening for chlamydial infection in women attending a family planning clinic. West Journal of Medicine, 138, 375-379.

Schellekens, J.F.P., Schouls, L.M. \& Laar, M.J.W. van de, (1993). Syfilis. In M.J.W. van de Laar (Ed). SOA in Nederland, Unpublished report, Bithoven: RIVM.

Scitovsky, A. \& Over, M. (1988). AIDS, costs of care in developed and the developing world, AIDS 2 (suppt. 1), 71-81.

Schlenk, E.A. \& Hart, K.L. (1984). Relationship between health locus of control, health value, and social support and compliance of persons with diabetes. Diabotes Care, 7 , 566-574.

Schweizer, A.T. (1984). Klantgerichtheid in een ziekenhuis. Tijdschritt voor Marketing, 18 (2) 70-79.

Severijnen, A.J. (1993). Chlamydia trachomatis als volksgezondheidsprobleern: prevalentie, epidemiologie, complicaties, kosten en scireening. Unpublished report, Rotterdam: Erasmus Universiteit, Instituut Maatschappelijke Gezondheidszorg.

Severijnen, A.J. \& Huisman, J. (1992). Epiderniologie van Chlarnydia trachomatis infecties in Nederland. SOA Bulletin, 13(6), 4-5.

Severijnen, A.J., Steyerberg E.W. \& Huisman J. (1992). Chlamydia trachomatis infectie complicaties, kosten, en effecten van screening, SOA Bulletin, 13(6), 19-21.

Shepperd, S.L., Solomon, L.J., Atkins, E. Foster, R.S. \& Frankowski, B. (1990). Determinants of breast self examination arnong women of lower income and lower education. Joumal of Behavioral Medicine, 13(4), 359-371.

Sherr, L., Strong, C. \& Goldmeler, D. (1990). Sexual behavior, condom use and prediction In attenders at Sexually Transmitted Disease Clinics: implications for counselling. Counselling Psychology Quarterly, 3, 4, 343-352.

Slegel, K., Chen, J.Y., Messango, F. \& Christ, G. (1987). Persislence and change in sexual behavior and perceptions of risk for AIDS among homosexual men. Presented at the 3rd International conference on AIDS, Washington DC.

Simon, K.J. \& Das, A. (1984). An application of the Health Belief Model toward an educational diagnosls for VD education. Heatth Education Quarterly, 11, 403-418.

SOA-Stlchting (1986). Kruis en Munt, Utrecht, SOA-Stichting.

SOA-stlchting (1988). Vrij vellig op vakantie. Utrecht, Brochure.

SOA-Stichting (1990). Pegistratie niet-curatieve SOA-bestrịding bij GGD'en: landelijke tabellen 1984-1990, SOA Stichting, Utrecht.

Spencer, B. (1990). Promoling use of the condom: the contribution of marketing. In N. Spira, B. Spencer, J.P. Moatti \& E. Bouvet. Public Health and the sexual transinission of diseases, direction for future research and health policy (pp 234-246). London: John Libbey Eurotext.

Spence, M.R., Adler, J. \& Mclellan, R. (1990). Pelvic Inflammatory Disease in the adolescent, Journal of Adolescent Health Care, 11, 304-309.

Stadlander, M. \& Kok, G. (1989). Prevention of Sexually Transmitted Diseases: relapse behavior of visitors to venereal disease clinics in Amsterdam. Health Education Research, 4(3), 267-271.

Stone, K.M., Grimms, D.A. \& Magder, L.S. (1986). Primary prevention of Sexually Transmitted Diseases. Journal of the American Medical Association, 255, 1763-1766. 
Stretcher, V.J., De Vellis, M., Brecker, M.H. \& Rosenstock I.M. (1986). The role of selfefficecy in achieving health behavior change. Health Education Quarterly, 13, 73-91.

Strunin, L \& Hingson, R. (1987). AIDS and adolescents: Knowligdge, beliefs, attitudes and behavior. Pediatrics, 79, 825-828.

Suchman, E.A. (1965). Stages of lilness and medical care. Joumal of Health and Social Behavior, 6, 114-120.

Tamburini, M., Massara, G., Bertario, L., Alberto, R. \& Putio, S. (1981). Usefulness of breast self examination for an early detection of breast cancer. Tumori, 67, 219-224.

Timko, C. (1987). Seeking medical treatment for breast cancer symptorns: determinanite of intentions to engage in prompt or delay behavior. Health Psychology, 6(4), 305-328.

Thompson, A.P. (1983). Extramarrital sex: a review of the research literature. The Journal of Sex Research, 19, 1-21.

Turtle, A.M., Ford, B. \& Habgood, A. (1989). AIDS related beliefs and behaviors of Australian university students. Medical Journal of Australia, 150, 371-376.

Valdiserri, R.O., Arena, P.J., Proctor, D. \& Bonnati, I. (1989). Variables influencing condom use in a cohort of gay and bisexual men. American Joumal of Public Health, 78, 801805.

Varnhagen, C.K., Svenson, LW., Godin, A.M., Johnson, L \& Salmon, T. (1991). Sexually Transmitted Diseasess and condoms: High School students' knowledge, attitudes and behavior. Canadian Journal of Public Health, 82, 129-132.

Velde, F.W. van der \& Pligt, J. van der (1991). AIDS related health behavior: coping, protection motivation and previous behavior. Journal of Behavioral medicine,

Velde, F.W. van der, Pligt, J. van der \& Hooykaas, C. (1992). Risk perceplion and behavior: Pessimism, realism and optimism about AIDS related health behavior. Psychology and Health, 6, 1/2, 23-38.

Vogels, T. \& Viet, R.W.F. van der (1990). Jeugd en seks. Den Haag: SDU.

Vogels, T. \& Danz, M.J. (1990). De intentie tot veilig seksueel gedrag. In Vogels T. \& R.W.F. van der Vliet, (Eds). Jeugd en Seks, Den Haag: SDU.

Vries, H. de., Weits, W., Dijkstra, M. \& Kok, G. (1992). The utilization of Qualitative and Quantitative data for health education programm planning, implementation, and evaluation: A spiral approach. Health Education Quarterly, 19(1), 101-115.

Vries, H. de, Dijkstra, M. \& Kuhlman, P. (1988). Self efficacy: the third factor besides attitudes and subjective norm as a predictor of behavioral intentions. Health Education Research, 3, 273-282.

Vries, $K$ de (1988). Campagne 'vrij veilig op vacantie'. SOA-bulletin, 9 (3) 8-9.

Vroome, E.M.M. de, Paalman, M.E.M., Sandfort, Th. G.M., Sleutjes, M., Vries, K.J.M. de \& Tielman, R.A.P. (1990). AIDS in the Netherlands: the effects of several years of campaining. International Journal of STD and AIDS, 1, 268-275.

Wallston, B.S. \& Wallston, K.A. (1987). Locus of Control and health: a review of literature. Health Education Monographs, 6, 107-117.

Washington, A.E., Aral, S.O., Wolner-Hansen, P., Grimes, D.A. \& Holmes, K.K.(1991). Assessing riskfactors for PID and its sequelea. Journal of the American Medical Assoclation, 266(18), 2581-2586.

Washington, A.E., Cates, W. \& Wasserheit, M. (1991). Preventing Pelvic Inflammatory Disease. Joumal of the American Medical Association, 266,18, 2574-2580. 
Washington, A.E. Katz P. (1991). Cost and payment source for Pelvic Inflammatory Disease: trends and projections, 1983 through 2000. Journal of the American Medical Association, 266(18), 2565-2569.

Water, H.P.A. van de, Wiggers, C.C.M., Bijleveld, C.C.J. Berkane, M. (1990). Schatting van het aantal HIV seropositieven in Nedertand: implicaties voor het toekomstig verloop van de AIDS epidemie. Nederlands Tijdschrift voor Geneeskunde, 134, 2482-2486.

Weinstein, N.D (1990). Perceptions of personal susceptibility to harm. In V.M. Mays, W. George, S.G. Schneider (Eds). Primary prevention of AIDS: psychological approachess.

Weinstein, N.D. (1980). Unrealistic optimism about future life events. Journal of Personality and Social Psychology, 39, 806-820.

Weinstein, N.D. (1982). Unrealistic optimism about susceptibility to health problems. Journal of Behavioral Medicine, 5, 441-460.

Weinstein, N.D. (1983). Reducing unrealistic optimism about illness susceptibility. Psychology and Heatth, 2, 11-20.

Weinstein, N.D. (1984). Why it won't happen to me: perceptions of risk factors and susceptibility. Psychology and Health, 3, 431-457.

Weinstein, N.D. (1993). Testing four competing theories of health protective behavior. Health Psychology, 12 (4), 324-333.

Wellings, K. (1986). AIDS and the condom. British Medical Journal, 293, 199-200.

Westrom, L. (1980). Incidence, prevalence and trends of acute Pelvic Inflammatory Disease and its consequences in industrialized countries. Joumal of Obstetric and Gyneacology, 138, 880 .

Westrom, L., Joesoef, R., Reynolds, G., Hagdu, A. \& Thompson, S.E. (1992). Pelvic Inflammatory Disease: a cohort study of 1844 women with laparascopically verified dlsease and 657 control women with normal laparoscopie results. Sexually Transmitted Diseases, 19, 185-192.

Westrom, L., Svensson, L., Wolner-Hansen, P. \& Mardh, P.A. (1982). Chlamydial and gonococcal Infection in a defined population of women. Scandinavian Journal of Infectious Diseases, 32 (suppl), 157-162.

Wijnsma, P. (1990). Adolescents in and outside schools. In: Paalman M. (ed). Promoting safer sox. Amsterdam: Swets \& Zeitlinger.

Wigersma, L. (1990). Huisartsgeneeskundig handelen bij klachten over de geslachtsorganen on de seksualiteit. Dissertatie, Amsterdam: meditekst.

Wht, J. de, Kok, G., Timmermans, P. \& Wijnsma, P. (1990). Determinanten van veilig vrijen en condoomgebruik bij jongeren. Gedrag en Gezondheid, 18 (3) 121-133.

Wh, J. de, Kok, G.J., Timmermans, C. \& Wijnsma, P. (1989). De effecten van het voorlichtingsprogramma 'AIDS, het komt je niet aanwaaien'. Unpublished report, GVO cahier 39, Maastricht: Pijksuniversiteit Limburg.

Wit, J.B.F. de, Griensven, G.J.P. van, Sandfort, T.G.M. \& Coutinho, R.A. (1993). Toename van onveilig seksueel gedrag onder homoseksuele mannen in Amsterdam. Nederlands Tijdschrit voor Geneeskunde, 137, 4, 209-210.

World Health Organization (1986). WHO Expert Committee on Venereal Diseasess and Treponematosen, Technical Report Series no. 736, Geneva. 
World Health Organization (1985). Simplified approaches for Sexually Transinitted Diseases control at the primary health care level. WHO document, WHONDT/85.437. Geneva.

Woiner-Hansen, P., Eschenbach, D.A. \& Paavonen, J. (1990). Decreased risk of symptomatic chlamydial pelvic inflammatory disease associated with oral contraceptive use. Journal of American Medical Association, 263, 54-59.

Wolner-Hansen, P., Klviat, N.B. Holmes, K.K. (1990). Atypical Pelvic Inflammatory Disease: subacute, chronic or subclinical upper genital tract infection in women. In K.K. Holmes, P.A. Mardh, P.F. Sparling and P.J. Wiesner (Eds.). Sexually Transmitted Diseases, 71-76 2nd ed New York, NY: McGraw-Hill.

Zessen, J.J. van \& Sandfort, Th. G.M. (1991). Seksualiteil in Nederland: seksueel gedrag, risico en preventie van AIDS. Amsterdam/Lisse: Swets and Zeitlinger. 


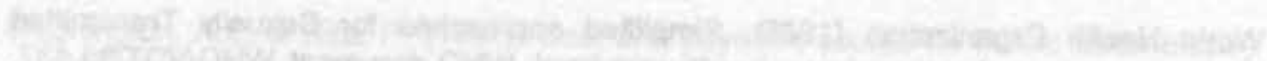

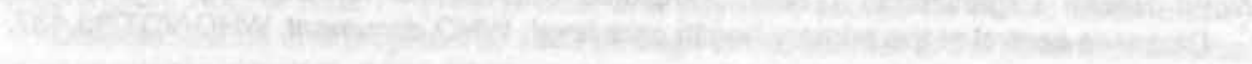

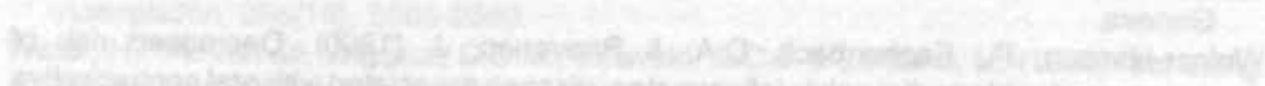

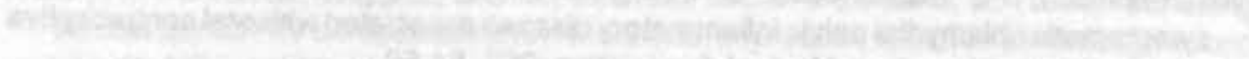

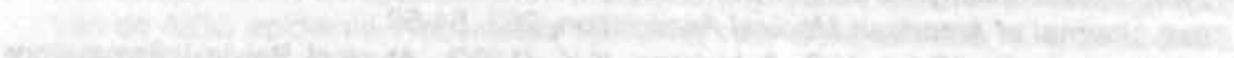
W0.

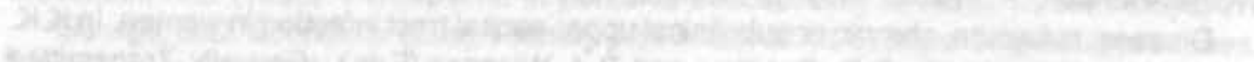

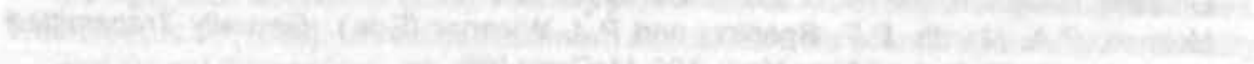
War:

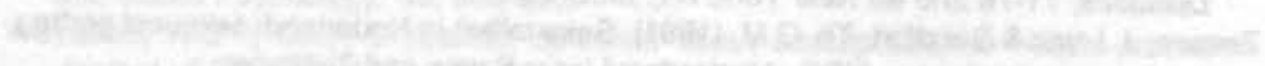

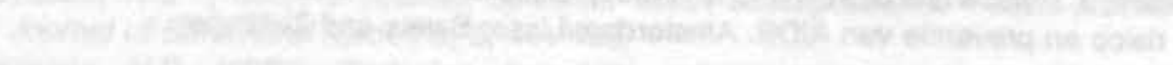

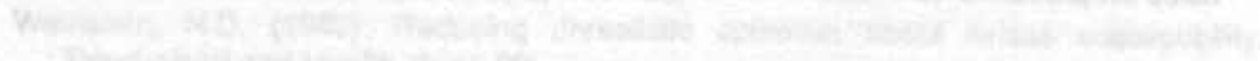

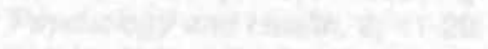

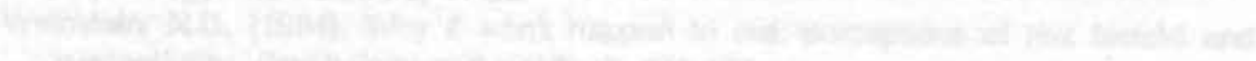

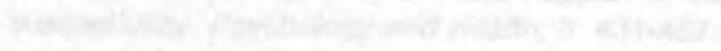

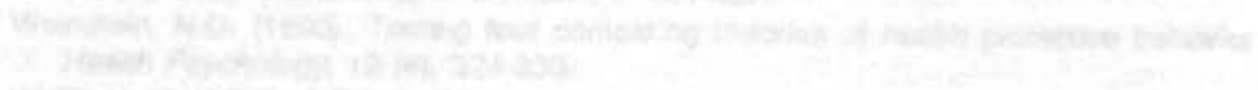

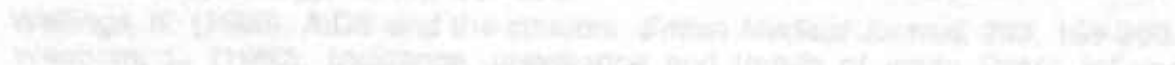

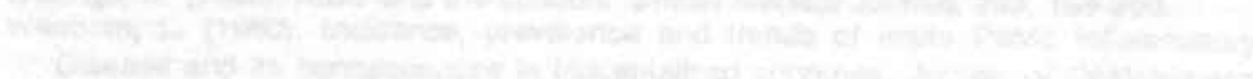

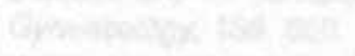

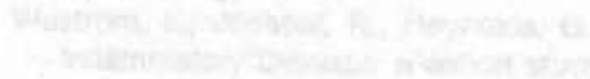

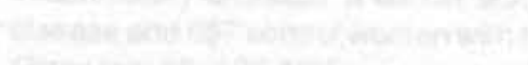

$x_{1}=\cos x$

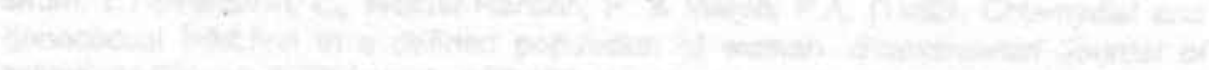

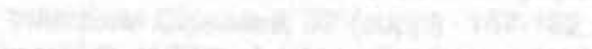

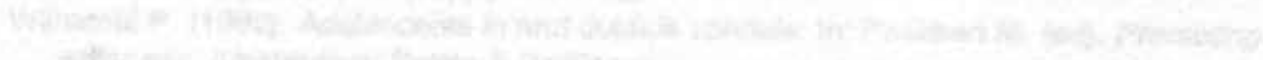

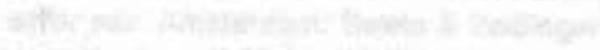

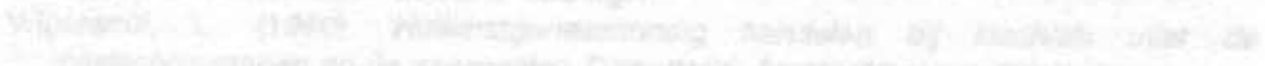

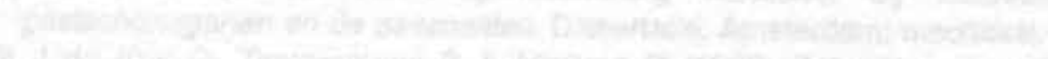

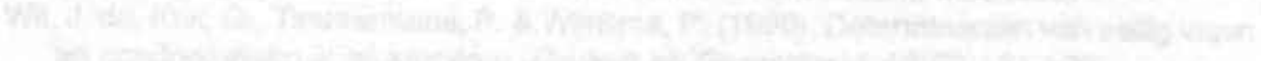

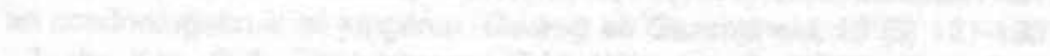

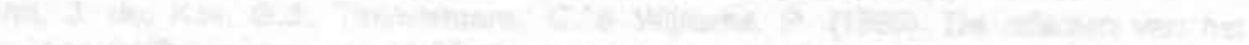

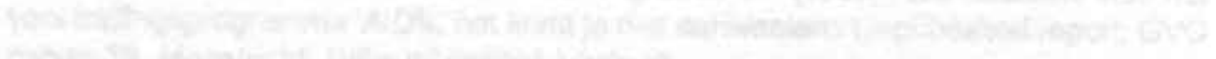

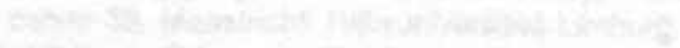

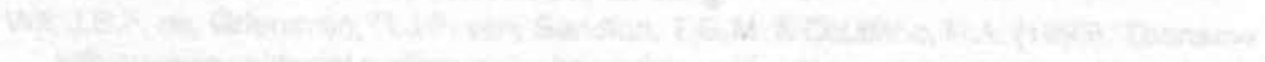

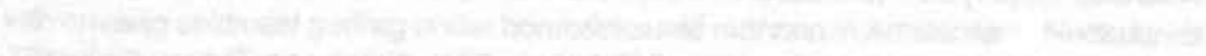

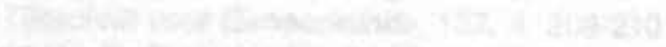

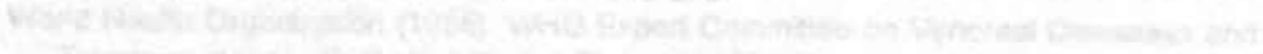

The 
SUMMARY IN ENGLISH

Interventions aimed at behavioural change and behavioural retention will have the greatest effect if they are developed exactly according to plan. A number of planning models can be used to that end, such as Green and Kreuter's health promotion planning model (1991) (PRECEDE-PROCEED model). This model has been succestully applied to several fields in the public health care (Green \& Kreuter, 1991).

This thesis focuses on the implementation of the first part of the PRECEDE. PROCEED model in the field of Sexually Transmitted Diseases (STDs). An analysis of the determinants of behaviour aimed at the primary prevention and early detection of STDs forms the essence of this thesis. These analyses lead to recommendations for the development of interventions aimed at primary prevention and early detection of STDs.

The thesis is divided into three parts. Part I gives an overview and starts off with the theoretical framework of the thesis. Second, a review is presented of national and international research into the determinants of behaviour aimed at the primary prevention and early detection of STDs. Third, the central question and the set-up of our studies are discussed. The most important findings of the studies are set forth in a general discussion, and are explored in great detail in parts II and III. Finally, part I is concluded with recommendations for the following fields: primary prevention, early detection, and the development of STD health services for those who suspect having an STD. Part II deals with the results of our studies into the determinants of sexual behaviour aimed at primary prevention of STDs. Part III covers the results of our studies into the determinants of behaviour aimed at early detection of STDs.

Chapter 1 starts with the adoption of the PRECEDE-PROCEED model in the STD field. The following parts come up for discussion: a social, epidemiological, behavioural and environmental diagnosis. In conclusion, the determinants of desired behaviour in an educational diagnosis are highlighted.

The description of the social diagnosis shows that not only the costs of the treatment (of complications) of STDs, but especially the emotional consequences of the complications, primarily subscribe the severity of these infectious disorders.

The epidemiological diagnosis demonstrates that there are fluctuations in the incidence of gonnorhea and syphilis. A considerable decrease has been reported in the incidence of these STDs among the Dutch population, especially in the last few years. However, the supposed underreporting and the lack of data about the incidence of other STDs lead to the necessity for careful statements about reducing the full magnitude of the problem. Likewise data show that the majority of the PID cases in the Netherlands is caused by gonorrhea and Chlamydia trachomatis infections. The epidemiological analysis concludes 
with the finding that primarily the following groups are at high risk of STD infection: men with homosexual contacts, adolescents and young adults, prostitutes and their clients and ethnic groups, such as Turks and Moroccans.

In the behaviour diagnosis we observed behaviour that, on the one hand, results in an increase in the risk of STDs (risk behaviour), such as unprotected Bexual exposures with varying partners, and, on the other hand, behaviour aimed at reducing the risk of STDs (STD preventive behaviour), such as condom use. In addition to a description of the risk groups, the prevalence of both types of behaviour is discussed per risk group.

In the educational diagnosis, the analysis of the determinants of the behaviours In question, the most important theoretical models that explain behaviour are briefly discussed, namely, the Theory of Reasoned Action (Fishbein \& Azjen, 1975), the Theory of Planned Behaviour (Azjen \& Madden, 1986), the Social Cognitive Learning Theory (Bandura, 1986), the Health Belief Model (Maiman \& Becker, 1974) and the Protection Motivation Theory (Rogers, 1983). Concepts from the various models are classified according to the Predisposing, Enabling and Feinforcing factors, as described in Green and Kreuter's PRECEDE-PROCEED model (1991). In a review of the research literature the role of various Predisposing. Enabling and Reinforcing factors with condom use was studied among several groups. It emerged that knowledge about STDs does not play a role in actual condom use. The literature study also shows that the following factors do contribute to the explanation of (the intention of using condoms in most cases: attitude towards condom use, expected effectiveness of condom use, previous condom use and self-efficacy expectations. There is a discrepancy in the findings with respect to the role of several factors in condom use, such as the perceived vulnerability to an STD, expected severity of an STD, social influence and beliefs about the effectiveness of condom use.

In a review of the research literature about the influence of Predisposing, Enabling and Reinforcing factors in STD self-examination behaviour, periodic clinical examination and prompt help-seeking behaviour, it was found that, until now, little research has been conducted into the determinants of the aforementioned behaviours. Research into other self-examination behaviours does give some insight into possible determinants, such as attitudinal aspects of selfexamination, self-efficacy expectations and social influence.

Finally, the last part of Chapter 1 focuses on the central question of the thesis and presents an outline of the set-up of our studies among 270 adolescents, 149 heterosexuals requesting an HIV test and 855 persons seeking medical assessment of a suspected STD.

A general discussion is provided in Chapter 2. It starts with a description of the most important research findings from our studies. A summary of these findings is in line with the description of the most important research findings of Chapters 3 to 5 of part II and Chapters 6 to 9 of part III. The general discussion of 
Chapter 2 is based on these findings. To avoid repetition, we now discuss Chapters 3 to 5 of part II and the subsequent recommendations for interventions aimed at primary prevention.

First, Chapter 3 describes a study among 149 heterosexuals with multiple sexual partners who requested an HIV test at an STD clinic or at a Municipal Health Service. In conclusion we can say that a large number of the heterosexuals who sought an HIV test did not use condoms when they had sexual contact with multiple partners. Results show that self-efficacy expectations, Health Locus of Control, perceived threat of an HIV infection, beliefs about condom use as responsible behaviour, and beliefs about condoms as a sign of distrust contribute to the explanation of the intention to use condoms with a casualnew partner.

The second study, described in Chapter 4 , is an investigation into the determinants of condom use among 270 secondary school students. The results show that the intention of using condoms with a casual partner among males can primarily be explained by: previous condom use, beliefs about condom use with multiple partners, beliefs about condom use with an STD, the perceived behaviour effectiveness of condom use and the social influence of parents. Beliefs about the costs, the planning and the disruptive effect of condoms do not play a role in the intention of using condoms with a casual partner. Whereas among females, however, only perceived importance of condom use contributes to the prediction of intended condom use with casual partners. This means that other factors play a role in the condom use of females.

Chapter 5 sets forth an analysis of the determinants of STD-preventive behaviour among 270 secondary school students. It became clear that the intention to STD-preventive behaviour among sexually active adolescents can primarily be explained by previous preventive behaviour, beliefs about behaviour effectiveness of STD communication and the gender of the person in question. Among sexually non-active pupils, the intention to use condoms is explained by: the perceived importance and annoyance of STD communication, the perceived importance and annoyance of selective partner choice and the gender of the respondent.

The above results lead to recommendations for adolescents and hetereosexuals who requested an HIV test concerning the target group and the content of the interventions. First, it is advisable to gear the content of education to the sexual activities of adolescents. Furthermore, we recommend to differentiate between the content of the education for sexually active males and sexually active females. This can take place on the basis of the items discussed, such as attitudinal aspect of condom use and the communication about STDs with a sex partner. Finally, it is advisable to focus specifically on training skills, which can be necessary when actually using condoms. For heterosexuals requesting an HIV test, it is advisable to promote, especially during posttest counselling, 
condom usage by concentrating on self-efficacy expectations, attitudinal aspects of condom use and the severity of an HIV infection.

For the summary of the studies into the determinants of early STD detection, we now discuss Chapters 6 to 7 followed by a summary of the corresponding recommendations.

Chapter 6 starts with a presentation of the results of determinants of STD selfexamination and periodic clinical examination among 855 persons seeking treatment for an STD. STD self-examination is not common among persons who exhibit risk behaviour. Intentions to STD self-examination are primarily explained by actually conducting self-examination (habit) and by the direct attitude towards the recommended behaviour. Components of the Health Belief Model, such as: perceived vulnerability and perceived severity of an STD do not play a role in the explanation of the intention to STD self-examination. The intention to periodic medical examination on STDs can especially be explained by the direct attitude towards periodic medical examination, the habit of regular check-ups for STDs and the perceived severity of and perceived vulnerability to an STD. Service attributes, including barriers of and benefits to a medical examination of STDs are of minor importance when explaining the intention of having regular STD check-ups.

A second study, presented in Chapter 7, concerns the delay behaviour of 627 persons with STD-related complaints of 855 respondents seeking medical assessment. Approximately one-quarter of those who have complaints delay' more than four weeks before seeking medical assessment of their symptoms. The four delay groups discerned (one week, one-two weeks, two-four weeks, and more than four weeks delay behaviour) differ from each other in: beliefs about physical consequences of STDs (infertility and skin injury), lack of need for consultation, symptoms attributed to specific sexual behaviour, partner pressure, information obtained through media messages and expectations about the existence of three service attributes (rapid cure, prescription of proper medication and the existence of walk-in consulting hours). Furthermore, habits in delay behaviour, shame/embarrassment and seeking the right physician play a role in the delay behaviour of people with complaints. Nevertheless, the amount of explained variance of the delay behaviour by these factors is limitec. The results of both studies lead to recommendations for the information given (during the consultation) to persons with multiple sexual partners, such as: recognising important symptoms, discussing the benefits of and barriers to STD self-examination, discussing aspects that promote prompt help-seeking behaviour and, finally, discussing the aspects that stimulate regular STD check-ups.

This summary continues with a presentation of the studies into the impontance of specific service attributes in STD services. Chapter 8 discusses the role of sixteen defined service attributes in the choice of services by persons suspecting an STD. A number of service attributes play an, albeit limited, role in the 
choice of STD clinics $(\mathrm{N}=627)$, the Family Planning Clinics $(\mathrm{N}=90)$ and the General Practitioners (GP) $(\mathrm{N}=108)$. The following service attributes are primarily involved: the existence of evening and weekend consulting hours, free treatment, option of a male or female physician, long tirne investrnent for seeking treatment for an STD, option of anonymous treatment with respect to insurance and the option of not having the GP notified of the STD examination. The perceived incompetence of the GP, shame/embarrassment and the long time investment led to persons, who previously consulted a GP, now sought treatment at an STD clinic.

Chapter 9 concerns a study into the role of the same sixteen service attributes in the intention of 270 adolescents to choose different services. The following service attributes play a role in the intention of choosing a Family Planning Clinic: the anonymous handling of the STD examination with regard to the GP, family and friends are not notified of the STD examination and the option of a male or female physician. The study also shows that secondary school students are not aware of the existence of low-threshold facilities for curative STD health care, such as STD clinics and the Municipal Health Service. Considering the research data, it is first of all advisable to give greater publicity to the existence of low-threshold STD clinics in the Netherlands. It is also advisable to consider a number of specific service attributes in the organisation of STD services, such as: anonymous treatment, professional treatment, walk-in consultating hours and evening and weekend consulting hours.

Finally, in the last part of Chapter 2 part I, several theoretical conclusions are drawn and recommendations made for future research. It was shown that the utility of the theoretical models is limited in explaining the behaviour aimed at primary prevention and early detection of STDs. Of course not all possible determinants of the various behaviours have been examined. Future research into condom use will have to be aimed primarily at finding out about the beliefs with respect to relational aspects on condom use. It is also advisable to study specific benefits of and barriers to STD self-examination and periodic medical examination more closely. Furthermore, it is advisable to not only expand research into patients' delay of recurrent attenders with other possible determinants (for instance, personality factors, sociological and cultural factors, defense mechanisms, dealing with complaints with other diseases), but to also link the patients delay to the ultimate STD diagnosis. 
incidentie van de overige SOA leiden tot de noodzaak terughoudend te zijn in uitspraken over een vermindering van de totale omvang van het probleem. Evenzo komt uit de gegevens naar voren dat het merendeel van de gevallen van Pelvic Inflammatory Disease (PID) in Nederland veroorzaakt wordt door infecties met gonorroe en Chlamydia Trachomatis. De epiderniologische analyse wordt afgesloten met de bevinding dat er op het terrein van de SOA voornamelijk sprake is van de volgende risico groepen: mannen met homoseksuele contacten, adolescenten en jong volwassenen, prostituees en hun clienten en etnische groeperingen, zoals Turken en Maroccanen.

In de gedragsdiagnose is enerzijds het gedrag bekeken wat resulteert in een toename van het risico op SOA (risico gedrag), zoals onbeschermde seksuele contacten met wisselende partners. Anderzijds is ingegaan op gedrag gericht op een vermindering van het risico op SOA (SOA-preventief gedrag), zoals condoomgebruik. Naast een beschrijving van de risico groepen is per risico groep het voorkomen van beide type gedragingen besproken.

In de educationele diagnose, de analyse van de determinanten van de betrokken gedragingen, komen allereerst de belangrijkste theoretische modellen ter verklaring van gedrag beknopt aan de orde, te weten: de theorie van het beredeneerd gedrag (Fishbein \& Ajzen, 1975), de theorie van het geplande gedrag (Ajzen \& Madden, 1986), de Sociaal Cognitieve Leertheorie (Bandura, 1986), het Health Belief Model (Maiman \& Becker, 1974) en de Protectie Motivatie Theorie (Rogers, 1983). Concepten uit de verschillende modellen zijn geordend onder de Predisposing. Enabling en Reinforcing factors, zoals beschreven in het PRECEDE.PROCEED model van Green en Kreuter (1991). In een review van de onderzoeksliteratuur is de rol van verschillende Predisposing, Enabling on Reinforcing factors bij condoomgebruik onder verschillende groeperingen onderzocht. Naar voren komt dat kennis over SOA geen enkele rol speelt bij het daadwerkelijk gebruiken van condooms. Ook komt uit het literatuuronderzoek naar voren dat de volgende factoren in de meeste gevallen wel een bijdrage leveren aan de verklaring van (de intentie tot) condoomgebruik: attitude tegenover condoomgebruik, verwachte effectiviteit van condoomgebruik, eerder condoomgebruik en self-efficacy expectations. Ten aanzien van de bijdrage van enkele factoren bij de verklaring van (de intentie tot) condoomgebruik is er sprake van inconsistentie in de bevindingen, zoals de afwezigheid van de invloed van vermeende vatbaarheid voor een SOA, vermeende ernst van een SOA, sociale invloed en opvattingen over de effectiviteit van condoom gebruik bij de intentie condooms te gebruiken.

In een review van de onderzoeksliteratuur naar de invloed van Predisposing, Enabling en Reinforcing factoren bij zelf-onderzoek op SOA, periodiek klinisch onderzoek en direkt hulpzoekgedrag is vastgesteld dat er tot op heden weinig onderzoek is verricht naar de determinanten van de laatstgenoemde gedragingen. Wel levert onderzoek naar andere zelfonderzoek gedragingen enig zicht 
op mogelijke determinanten, zoals attitudinale aspecten van zelfonderzoek, selfefficacy expectations en sociale invloed.

Tot slot is in het laatste gedeelte van hoofdstuk I nader ingegaan op de centrale vraagstelling van de dissertatie en wordt tevens globaal de opzet van het door ons uitgevoerd onderzoek onder 270 adolescenten, 149 heteroseksuelen met een HIV-testverzoek en 855 personen met een SOA-hulpvraag gepresenteerd.

Hoofdstuk 2, een algemene discussie, vangt aan met een beschrijving van de belangrijkste bevindingen van het door ons uitgevoerd onderzoek. Een samenvatting van deze bevindingen komt overeen met de weergave van de belangrijkste onderzoeksbevindingen van de hoofstukken drie tot en met vijf van deel II en hoofstuk zes tot en met negen van deel III. Om herhaling te voorkomen bespreken we nu allereerst de hoofdstukken 3 tot en met 5 van deel II en de daaruit volgende aanbevelingen ten behoeve van interventies gericht op primaire preventie van SOA.

Hoofdstuk 3 gaat over een onderzoek onder 149 heteroseksuelen met wisselen. de seksuele partners die een HIV test aanvragen bij een drempelvrije SOA. polikliniek of GGD. Geconcludeerd is dat een groot gedeelte van de heteroseksuelen met een HIV testverzoek geen condooms gebruikt bij seksueel contact met wisselende partners. De resultaten tonen dat self-efficacy expectations, Health Locus of Control, waargenomen dreiging van een HIV infectie, opvattingen over condoomgebruik als verantwoord gedrag en opvattingen over condooms als een teken van wantrouwen een bijdrage leveren aan de verklaring van de intentie een condoom te gebruiken bij een losse/nieuwe partner.Hoofdstuk 4 beschrijt een studie naar determinanten van condoomgebruik onder 270 middelbare scholieren. De resultaten tonen dat de intentie tot condoomgebruik met een tijdelijke partner onder jongens hoofdzakelijk verklaard wordt door: eerder condoomgebruik, opvattingen over condoomgebruik bij wisselende partners, opvattingen over condoomgebruik bij de aanwezigheid van een SOA, de waargenomen gedragseffectiviteit van condoomgebruik en de sociale invloed van ouders. Opvattingen over de kosten, de planning en de verstorende werking van condooms spelen geen enkele rol bij de verklaring van de intentie condooms te gebruiken bij een losse partner. Bij meisjes daarentegen levert alleen het vermeende belang van condoomgebruik een bijdrage aan de voorspelling van de intentie tot condoomgebruik bij losse partners. Dit betekent dat andere factoren een rol spelen bij het condoomgebruik van meisjes.

Hoofdstuk 5 betreft een analyse van determinanten van SOA-preventief gedrag onder $\mathbf{2 7 0}$ middelbare scholieren. Naar voren komt dat de intentie tot SOApreventief gedrag onder de groep seksueel actieve adolescenten met name verklaard wordt door eerder preventief gedrag, opvattingen over de gedragseffectiviteit van communicatie over SOA en het geslacht van de betrokkene. Bij seksueel niet-actieve leerlingen wordt de intentie tot condoomgebruik verklaard door: het ervaren belang en de vervelendheid van communicatie over SOA, het 
ervaren belang en de vervelendheid van selectieve partnerkeuze en het geslacht van de respondent.

De resultaten leiden tot aanbevelingen ten behoeve van adolescenten en heteroseksuelen mel een HIV testverzoek aangaande de indeling van doelgroepen en de inhoud van interventies. Zo is het wenselijk om de inhoud van de voorlichting af te stemmen op de seksuele activiteit van de adolescenten. Daarnaast verdient het aanbeveling om differentiatie aan te brengen in de inhoud van de voorlichting aan seksueel actieve jongens en seksueel actieve meisjes. Afstemming van de inhoud kan plaatsvinden aan de hand van de besproken items, zoals de attitudinale aspecten van condoomgebruik en de communicatie over SOA. Tot slot is het wenselijk om met name aandacht te besteden aan een training in vaardigheden, welke noodzakeliịk kunnen ziịn bij het daadwerkelijk gebruiken van condooms. Ten behoeve van heleroseksuelen met een HIV testverzoek verdient het aanbeveling om condoomgebruik te bevorderen door met name tijdens posttest counselling in te gaan op attitudinale aspecten van condoomgebruik, de ernst van een HIV infectie en self-efficacy expectations.

Ten behoeve van de samenvatting van het onderzoek naar de determinanten van early STD detection bespreken we nu eerst de hoofdstukken 6 en 7 met daaraan aansluitend een samenvatting van de bijbehorende aanbevelingen.

Hoofdstuk 6 vangt aan met de weergave van de resultaten naar determinanten van zelfonderzoek op SOA en periodiek medisch onderzoek onder 855 personen met een SOA hulpuraag. Zelfonderzoek op SOA is geen gewoonte bij mensen die risico gedrag vertonen. De intentie tot zelfonderzoek op SOA wordt hoofdzakelijk verklaard door het reeds uitvoeren van zelfonderzoek (gewoonte) en door de direkte attitude tegenover het aanbevolen gedrag. Componenten van het Health Belief Model, zoals: waargenomen vatbaarheid en waargenomen ernst van een SOA spelen geen rol bij de verklaring van de intentie tot zelfonderzoek op SOA. De intentie tot periodiek medisch onderzoek op een SOA wordt met name verklaard door de direkte attitude tegenover periodiek medisch onderzoek op een SOA, de gewoonte om zichzelf regelmatig op SOA te laten onderzceken en de waargenomen ernst en waargenomen vatbaarheid voor een SOA. Servicekenmerken, waaronder barrieres en voordelen van medisch onderzoek op SOA spelen een ondergeschikte rol bij de verklaring van de intentie zichzelf regelmatig op SOA te laten onderzoeken.

Een tweede studie, in hoofdstuk 7 weergegeven, betreft het uitstelgedrag van 627 personen die in verband met klachten hulp vragen voor onderzoek op SOA. Ongeveer een kwart van de personen met klachten wacht langer dan vier weken alvorens medische hulp te zoeken in verband met klachten. De vier onderscheiden uitstelgroepen (1 week, 1-2 weken, 2-4 weken en meer dan 4 weken uitstelgedrag) verschillen van elkaar met betrekking tot: opvattingen over de fysieke gevolgen van een SOA (infertiliteit en huidafwijkingen), gemis aan consumptienoodzaak, de mate waarmeesymptomen aan seksueel gedrag 
worden geattribueerd, druk van de partner, informatie verkregen via media en verwachtingen over de aanwezigheid van drie servicekenmerken (snel genezen zijn, juiste medicatie krijgen en de aanwezigheid van een inloopspreekuur). Daarnaast spelen gewoonten in uitstelgedrag, schaamte en het zoeken naar een goede arts een rol bij het uitstelgedrag van personen met klachten. Echter de hoeveelheid verklaarde variantie van het uitstelgedrag door deze factoren is beperkt. De resultaten van beide studies leiden tot aanbevelingen ten behoeve van de voorlichting (tijdens het consult) aan personen met wisselende seksuele contacten, zoals: de herkenning van belangrijke symptomen, de bespreking van voor en nadelen van zelfonderzoek op SOA, de bespreking van aspecten die direkt hulpzoekgedrag bevorderen en tot slot de bespreking van de aspecten die regelmatige controle op SOA stimuleren.

Deze samenvalting wordt vervolgd met een weergave van het onderzoek naar het belang van specifieke servicekenmerken in de SOA hulpverlening. Hoofdstuk 8 gaat in op de rol van zestien omschreven servicekenmerken bij de keuze voor voorzieningen van mensen met vermoeden van een SOA. Een aantal servicekenmerken speelt een, zij het beperkte rol, bij de keuze voor een drempelvrije SOA polikliniek ( $N=627)$, het Rutgershuis $(N=90)$ en de huisarts $(N=10$ 8). Het gaat daarbij met name om de volgende kenmerken van de hulpverlening: de aanwezigheid van avond- en weekend spreekuren, gratis behandeling, keuze tussen een mannelijke en vrouwelijke arts, lange tijdsinvestering van een SOA hulpvraag, anonieme behandelmogelijkheid ten aanzien de verzekering en de mogelijkheid om de huisarts niet op de hoogte te stellen van de SOA hulpvraag. De waargenomen ondeskundigheid van de huisarts, schaamte en de lange tijdsinvestering heeft ertoe geleid dat personen die éérder een huisarts consulteerden nu bij een drempelvrije SOA polikliniek hulp hebben gezocht.

Hoofdstuk 9 betreft een studie naar de rol van dezelfde zestien servicekenmerken bij de intentie tot keuze voor verschillende voorzieningen van 270 middelbare scholieren. De volgende servicekenmerken spelen een rol bij de intentie tot keuze van middelbare scholieren voor een Rutgershuis: de anonieme verwerking van de hulpvraag ten aanzien van de huisarts, familie en vrienden komen niet op de hoogte van de hulpvraag en de mogelijkheid om een mannelijke dan wel vrouwelijke arts te kiezen. Tevens toont het onderzoek dat middelbare scholieren onbekend zijn met de aanwezigheid van laagdrempelige voorzieningen voor curatieve SOA bestrijding, zoals drempelvrije SOA poliklinieken en de GGD. Gezien de onderzoeksgegevens is het allereerst wenselijk om meer bekendheid te geven over de aanwezigheid van drempelvrije SOA poliklinieken in Nederland. Tevens verdient het aanbevelingen om bij de organisatie van het SOA hulpaanbod rekening te houden met een aantal specifieke servicekenmerken, zoals: anonieme behandeling, professionele behandeling, inloop spreekuren en avond- en weekend spreekuren. 
Tenslotte zijn in het laatste onderdeel van hoofdstuk 2 enkele theoretische conclusies getrokken en zijn aanbevelingen geformuleerd ten behoeve van toekomstig onderzoek. Naar voren is gekomen dat de bruikbaarheid van theoretische modellen bij de verklaring van gedrag gericht op primaire preventie en vroegtijdige ontdekking van SOA beperkt is. Uiteraard zijn niet alle mogelijke determinanten van de verschillende gedragingen onderzocht. Toekomstig onderzoek naar condoomgebruik zal met name gericht moeten worden op het achterhalen van opvattingen die betrekking hebben op de relationele aspecten van condoomgebruik. Zo ook is het wenselijk om specifieke voor- en nadelen van zelfonderzoek op SOA en periodiek medisch onderzoek nader te bestuderen. Verder is het gewenst om niet alleen onderzoek naar patients' delay van herhaalde bezoekers uit to breiden met andere mogelijke determinanten (b.v. persoonlijkheidskenmerken, defensiemechanismen, sociaal culturele factoren, het omgaan met klachten in het algemeen) van lang uitstelgedrag, maar ook het patients' delay te koppelen aan de uiteindelijke SOA-diagnose. 


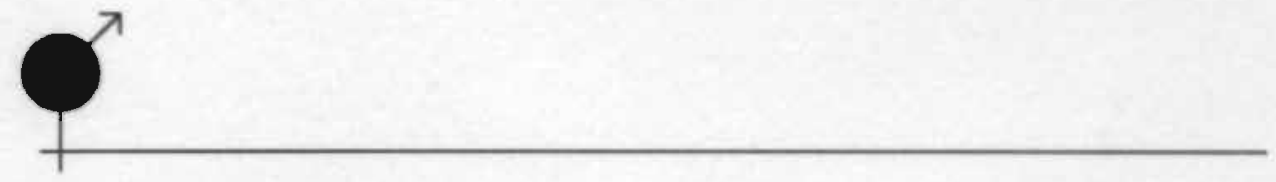

\section{CURRICULUM VITAE}

Ellie Leenaars was born in Gilze Rijen on Octobre 29nd, 1953. After finishing secundary school she worked as a nurse, later as a charge-nurse, in a general hospital in Tilburg. She left this job in 1981 and started her studies in Psychology at the University of Brabant in Tilburg. In 1986 she graduated in Clinical and Health Psychology. Specific attention was paid to psychological aspects of myocardial infarction on the quality of life of patients and their partners. As follows she received a basic training in behaviour therapy (NVP registration). This path changed in 1987 when she started at the Department of Health Sciences and Epidemiology at the University of Utrecht with a research project about Sexually Transmitted Diseases. A part of this work resulted in the articles, edited in this book. Since 1993 another challenge has been found. After a scientific period she became a forensic psychologist at a penitentiary in Breda. Now she is occupied with diagnosis and short term behavioural interventions in prisoners with mental disorders. 
